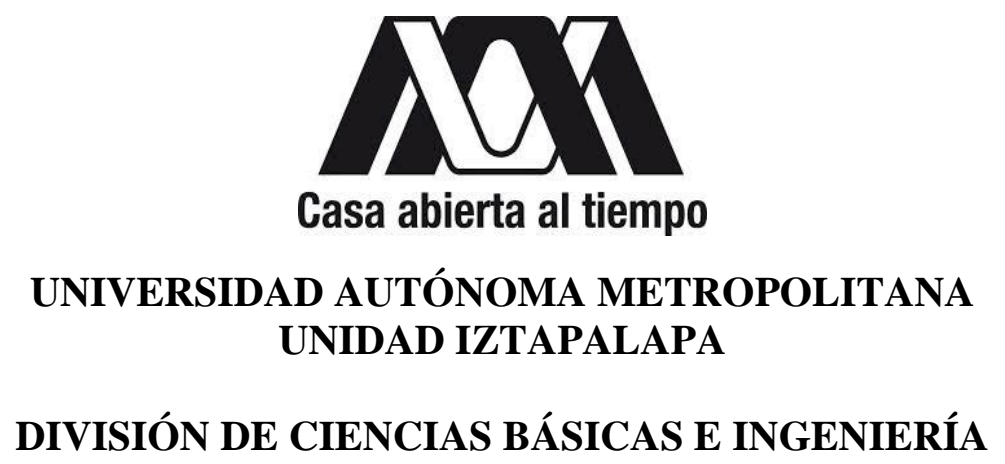
EFECTOS DE CONFINAMIENTO ESPACIAL Y DE NÚCLEO DE TAMAÑO FINITO EN SISTEMAS ATÓMICOS

\author{
Tesis que presenta \\ Rafael Alejandro Rojas Calderón \\ Para obtener el grado de \\ Doctorado en Ciencias (Física)
}

Asesor:

Dr. Norberto Aquino Aquino

Jurado calificador:
Presidente:
Dr. Salvador Antonio Cruz Jiménez
Secretario:
Dr. Norberto Aquino Aquino
Vocal:
Dr. Eleuterio Castaño Tostado
Vocal:
Dr. Andrei Solórzano Pérez
Vocal:
Dr. Antonio Flores Riveros

Ciudad de México, a 24 de julio de 2019 
"Después de todo, ¿qué es un científico entonces? Es un hombre curioso que mira a través del ojo de una cerradura, la cerradura de la naturaleza, tratando de saber qué es lo que sucede"

Jacques Yves Cousteau 


\section{Agradecimientos}

Quiero agradecer a mi papá, el Dr. Rafael Rojas Rodríguez, por guiarme en el camino de la Física y de la vida. A mi mamá, la Dra. Ma. Guadalupe Calderón Carranza, que siempre me ha brindado su amor y apoyo incondicional. A mis hermanos, David Rojas Calderón y Roberto Arturo Rojas Calderón por estar a mi lado apoyándome y dándome su amistad. Y al Amor y Luna de mi vida, la Dra. Ma. del Socorro Rosas López, por todo su apoyo y amor que me ha dado durante la elaboración de esta tesis.

Les agradezco a mis amigos y compañeros, que siempre están para alegrarme y animarme.

Le agradezco a mi asesor, el Dr. Norberto Aquino Aquino por su valioso tiempo y por brindarme la oportunidad de trabajar con él. Además le doy las gracias por todo su apoyo durante la elaboración de este trabajo y por los conocimientos que adquirí bajo su tutela.

También deseo expresar mi agradecimiento a los miembros del jurado por sus comentarios y observaciones sobre este trabajo.

Agradezco a la Fundación Alberto y Dolores Andrade por su apoyo durante tantos años de mi vida académica.

Finalmente pero muy importante, agradezco a la Universidad Autónoma Metropolitana, por darme la oportunidad de participar en su programa de becas de posgrado, y además por abrirme sus puertas y darme la oportunidad de adquirir muchos conocimientos y experiencias tanto de mi profesión como de la vida.

Por esto y mucho más les estaré muy agradecido. 


\section{Resumen}

Se estudia el comportamiento de distintos sistemas atómicos cuando son sometidos a altas presiones externas: átomos hidrogenoides, de helio y litio. La presión ejercida se modela mediante el confinamiento espacial de los átomos, dentro de un pozo de potencial con simetría esférica, tanto de paredes impenetrables como penetrables.

Se estudian diversas propiedades de los sistemas cuánticos confinados. En el átomo de hidrógeno: la polarizabilidad, la presión, el desdoblamiento hiperfino, el apantallamiento magnético y la probabilidad de tunelamiento (en el caso de confinamiento dentro de paredes penetrables). En el átomo de helio: la polarizabilidad y los potenciales de ionización. En el átomo de litio: el efecto producido por el confinamiento sobre el estado base del sistema.

Para hallar la energía del estado base de los sistemas descritos anteriormente, se hace uso del método variacional de Rayleigh-Ritz. Para el átomo hidrogenoide confinado, también se resuelve de forma exacta la ecuación de Schrödinger, escribiendo la solución en términos de funciones hipergeométricas confluentes, y se calculan soluciones numéricas con muy alta precisión.

Además estudiamos algunas propiedades poco consideradas en la literatura. Por ejemplo, para el átomo de hidrógeno confinado: el espectro de estructura fina para el estado base y algunos estados excitados, y correcciones relativistas. Estudiamos el efecto de considerar un núcleo de volumen finito, en lugar de un núcleo de tamaño puntual (como habitualmente se utiliza), sobre la energía del estado base de sistemas atómicos confinados de uno, dos y tres electrones.

También estudiamos átomos exóticos confinados: los llamados átomos muónicos.

Se analiza el comportamiento de la entropía informática de Shannon en el espacio de coordenadas en los sistemas cuánticos confinados, y su posible aplicación como medida de la calidad de la función de onda, así como su uso como medida de la localización (o deslocalización) de las partículas dentro de un sistema confinado. 


\section{Abstract}

We study the behavior of different atomic systems (hydrogen-like atoms, helium and lithium) when they are subjected to high external pressures. The exerted pressure is modeled by spatial confinement of the atoms, inside a spherical potential for both impenetrable and penetrable walls.

We study different properties of the confined quantum systems. For a hydrogen atom, polarizability, pressure, hyperfine splitting, magnetic screening and the tunneling probability (for the case of confinement inside penetrable walls) are analyzed, whereas for helium, polarizability and ionization potentials. Also, confinement induced effects are studied for lithium ground state.

In order to analyze such effects for the above described systems, we use the Rayleigh-Ritz variational method. For a confined hydrogen-like atom, we solve the Schrödinger equation in an exact manner by writing the solution in terms of confluent hypergeometric functions, and we calculate numerical solutions with a very high accuracy.

We also study some properties seldom considered in the literature. For example, for the confined hydrogen atom, the fine structure spectrum for the ground and some excited states, in conjunction with relativistic corrections. In addition, we study effects arising from spatially extending the nuclear region through a finite volume occupied by the nucleus, instead of considering it a point size particle (as it is usually assumed), when analyzing the ground state energy of atomic systems of one, two and three electrons.

We also study exotic confined atoms, the so called muonic atoms.

We analyze the Shannon information entropy behavior in the coordinate space for quantum confined systems, viewed as an application for measuring the quality of the wave function, as well as its use as a measure of localization and delocalization of particles inside a confined system. 


\section{Índice general}

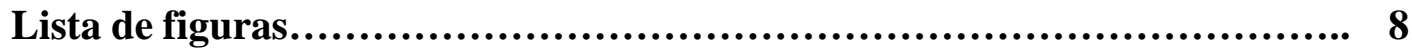

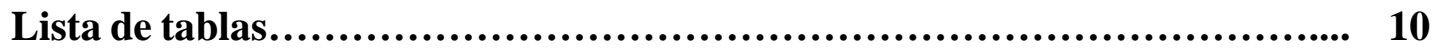

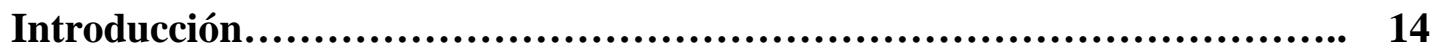

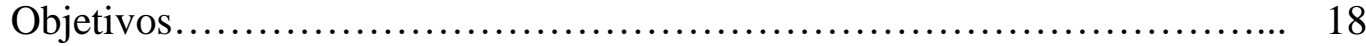

Organización de este trabajo............................................. 18

1. Átomo de hidrógeno confinado...................................... 20

1.1. Solución por el método variacional de Rayleigh-Ritz............ 20

1.1.1. AHC dentro de paredes impenetrables..................... 21

1.1.1.1. Casos donde la función de corte incluye funciones esféricas de Bessel............................... 23

1.1.1.2. Entropía informática de Shannon como medida de la calidad de la función de onda........................ 26

1.1.2. AHC dentro de paredes penetrables........................ 34

1.1.2.1. Propiedades físicas................................. 37

1.2. Solución exacta................................................. 46

1.2.1. AHC dentro de paredes impenetrables..................... 46

1.2.1.1. Estructura fina del AHC............................. 49

1.2.2. AHC dentro de paredes penetrables........................ 62

1.2.2.1. Entropía de Shannon y corrección de núcleo de tamaño finito........................................ 67

1.3. Conclusiones............................................... 80

2. Átomo de helio confinado......................................... 84

2.1. Solución por el método variacional de Rayleigh-Ritz............. 84

2.2. Propiedades físicas..................................................................... 92 
2.2.1. Polarizabilidad..................................................................... 92

2.2.2. Potenciales de ionización.......................................................... 94

2.2.3. Entropía informática de Shannon............................................. 99

2.2.4. Núcleo de tamaño finito......................................................... 104

2.3. Conclusiones..................................................................... 110

3. Átomo de litio confinado............................................................................ 112

3.1. Sistema no perturbado (núcleo puntual)....................................... 112

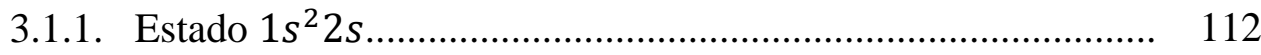

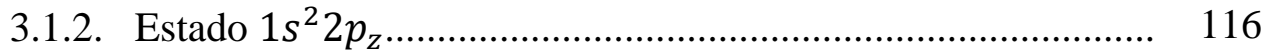

3.2. Corrección de núcleo de tamaño finito.......................................... 118

3.3. Conclusiones....................................................................... 126

4. Átomos muónicos confinados....................................................... 127

4.1. Modelo de átomo muónico confinado con núcleo puntual.............. 128

4.2. Átomos muónicos confinados con núcleo de tamaño finito............. 129

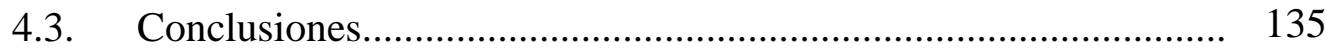

Conclusiones generales.................................................................................. 137

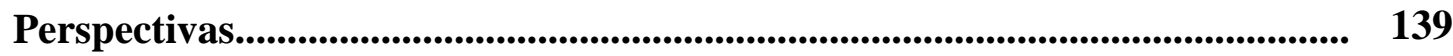

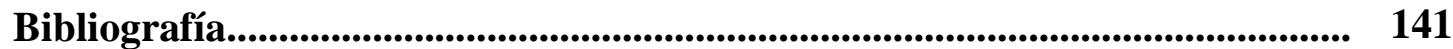

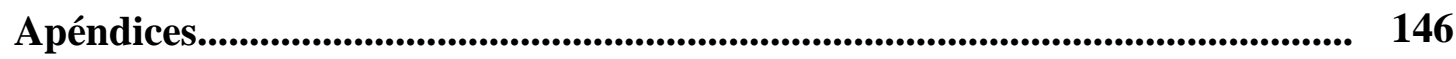

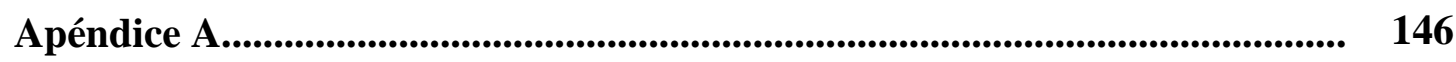

Código en Mathematica para calcular la energía del estado base del AHC dentro de una barrera esférica de paredes penetrables, usando el método variacional de Rayleigh-Ritz

Apéndice B.........................................................................................................................

Código en Mathematica para calcular en forma exacta las energías del AHC dentro de una barrera esférica de paredes penetrables

Apéndice $\mathbf{C}$.

Trabajos publicados 


\section{Lista de figuras}

1.1 Energía del estado base del AHC como función del parámetro $\alpha \ldots \ldots \ldots \ldots \ldots \ldots . . . .25$

1.2 Entropía de Shannon como función del radio de confinamiento.............. 33

1.3 Entropía de Shannon como función del radio de confinamiento............. 33

1.4 Constante de desdoblamiento hiperfino como función de $R_{c} \ldots \ldots \ldots \ldots \ldots \ldots .41$

1.5 Constante de apantallamiento magnético como función de $R_{C} \ldots \ldots \ldots \ldots . . . .42$

1.6 Polarizabilidad en la aproximación de Buckingham como función de $R_{c} \ldots \ldots . \quad 43$

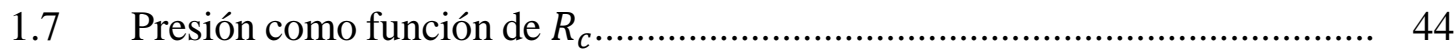

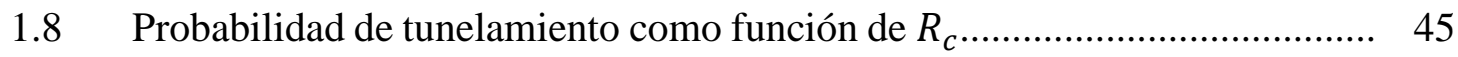

1.9 Correcciones relativistas a la energía cinética para los estados $1 s, 2 s, 2 p$ y

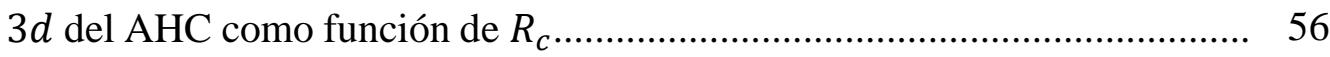

1.10 Término de Darwin para los estados $1 s$ y $2 s$ del AHC como función de $R_{c} . . \quad 57$

1.11 Acoplamiento espín-órbita $E_{s o}^{(1)}$ para los estados $2 p$ y $3 p$ del AHC como

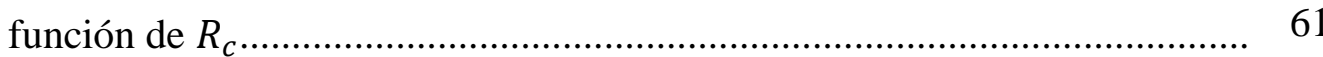

1.12 Acoplamiento espín-órbita para el estado 3d del AHC como función del radio de confinamiento.

1.13 Razón de la corrección a la energía del estado base del AHC sobre la corrección del átomo de hidrógeno libre, como función de $R_{c} \ldots \ldots \ldots \ldots \ldots \ldots \ldots . . . . . . .76$

1.14 Corrección de núcleo finito y entropía de Shannon como funciones del radio de confinamiento, para una barrera esférica impenetrable

1.15 Corrección de núcleo finito y entropía de Shannon como funciones del radio de confinamiento, para $V_{0}=0$ hartrees.

1.16 Corrección de núcleo finito y entropía de Shannon como funciones del radio de confinamiento, para $V_{0}=1$ hartrees 
1.17 Corrección de núcleo finito y entropía de Shannon como funciones del radio de confinamiento, para $V_{0}=5$ hartrees......

1.18 Función de onda radial dentro de la barrera, evaluada en el origen, como función del radio de confinamiento y la altura de la barrera.......................... 81

2.1 Polarizabilidad en la aproximación de Kirkwood como función de $R_{c} \ldots \ldots \ldots . . .92$

2.2 Energía del estado base del helio y del ion $\mathrm{He}^{+}$como función del radio de confinamiento, para una barrera de altura $V_{0}=5$ hartrees.

2.3 Potenciales de ionización del átomo de helio confinado como función del radio de confinamiento, para una barrera de altura $V_{0}=5$ hartrees

2.4 Entropía de Shannon del átomo de helio confinado, como función del radio de confinamiento.

2.5 Entropía informática de Shannon del átomo de helio y el ion $\mathrm{He}^{+}$ confinados, como función del radio de confinamiento

2.6 Corrección de núcleo finito a la energía del estado base del átomo de helio confinado, como función del radio de confinamiento y la altura de la barrera

3.1 Energía de los estados $1 s^{2} 2 s$ y $1 s^{2} 2 p_{z}$ del átomo de litio confinado, como función del radio de confinamiento.

3.2 Corrección de núcleo finito a la energía de los estados $1 s^{2} 2 s$ y $1 s^{2} 2 p_{z}$ del átomo de litio confinado, como función del radio de confinamiento, para el isótopo ${ }^{7} \mathrm{Li}$

3.3 Corrección de núcleo finito a la energía de los estados $1 s^{2} 2 s$ y $1 s^{2} 2 p_{z}$ del átomo de litio confinado, como función del radio de confinamiento, para el isótopo ${ }^{6} \mathrm{Li}$

4.1 Corrección de núcleo finito a la energía del estado base del átomo muónico confinado dentro de una barrera impenetrable de radio $R_{c}$, como función del radio de confinamiento

4.2 Razón de la corrección de núcleo finito del átomo muónico confinado y la corrección de núcleo finito del átomo muónico libre de confinamiento......... 


\section{Lista de tablas}

1.1 Energía del estado base del AHC, calculada usando diferentes funciones de corte

1.2 Energía del estado base del AHC, calculada usando diferentes funciones de corte.

1.3 Energía del estado base del AHC calculada por el método variacional usando la función de prueba $\psi_{t}=\left[c_{1} j_{0}\left(X_{10} r / R_{c}\right)+c_{2} j_{0}\left(X_{20} r / R_{c}\right)\right] e^{-\alpha r} .30$

1.4 Entropía de Shannon para cada función de prueba, como función del radio de confinamiento.

1.5 Entropía de Shannon para cada función de prueba, como función del radio de confinamiento.

1.6 Energía del estado base del AHC dentro de una caja penetrable de radio $R_{c}$ y altura de la barrera $V_{0}$

1.7 Término de contacto de Fermi, constante de apantallamiento magnético, polarizabilidad, presión y probabilidad de tunelamiento para el átomo de hidrógeno confinado en una esfera penetrable de radio $R_{c} \mathrm{y}$ una barrera de altura $V_{0}$

1.8 Espectro de energías del AHC dentro de barreras impenetrables, como función del radio de confinamiento

1.9 Espectro de energías del AHC dentro de barreras impenetrables, como función del radio de confinamiento

1.10 Corrección relativista y término de Darwin del estado base del AHC como función del radio de confinamiento

1.11 Corrección relativista y término de Darwin del estado $2 s$ del AHC como función del radio de confinamiento 
1.12 Corrección relativista y corrección de acoplamiento espín-órbita del estado $2 p$ del AHC como función del radio de confinamiento.

1.13 Corrección relativista y corrección de acoplamiento espín-órbita del estado $3 p$ del AHC como función del radio de confinamiento.

1.14 Corrección relativista y corrección de acoplamiento espín-órbita del estado $3 d$ del AHC como función del radio de confinamiento

1.15 Energía del estado base del AHC como función del radio de confinamiento y la altura de la barrera.

1.16 Energía de algunos estados excitados del AHC como función del radio de confinamiento y la altura de la barrera

1.17 Energía de estados excitados del átomo de hidrógeno confinado como función del radio de confinamiento y la altura de la barrera.

1.18 Corrección a la energía del estado base del AHC debida al núcleo finito, para una barrera de altura $V_{0}=0$ hartrees. Razón entre la corrección a la energía del sistema confinado y la corrección del átomo de hidrógeno libre, y entropía de Shannon

1.19 Corrección a la energía del estado base del AHC debida al núcleo finito, para una barrera de altura $V_{0}=1$ hartrees. Razón entre la corrección a la energía del sistema confinado y la corrección del átomo de hidrógeno libre, y entropía de Shannon.

1.20 Corrección a la energía del estado base del AHC debida al núcleo finito, para una barrera de altura $V_{0}=5$ hartrees. Razón entre la corrección a la energía del sistema confinado y la corrección del átomo de hidrógeno libre, y entropía de Shannon.

1.21 Corrección a la energía del estado base del AHC debida al núcleo finito, para una barrera de altura $V_{0}=10$ hartrees. Razón entre la corrección a la energía del sistema confinado y la corrección del átomo de hidrógeno libre, y entropía de Shannon.

1.22 Corrección a la energía del estado base del AHC debida al núcleo finito, para una barrera de altura $V_{0}=\infty$ hartrees. Razón entre la corrección a la 
energía del sistema confinado y la corrección del átomo de hidrógeno libre, 75 y entropía de Shannon

2.1 Parámetros variacionales óptimos $\alpha$ y $\gamma$, y energía del estado base del átomo de helio confinado como función del radio de confinamiento y la

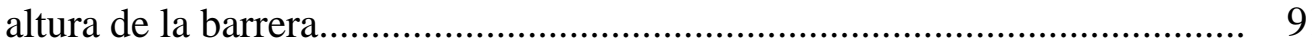

2.2 Polarizabilidad en la aproximación de Kirkwood como función de $R_{c} \ldots \ldots \ldots . .93$

2.3 Energía del estado base del $\mathrm{He}^{+}$como función del radio de confinamiento y la altura de la barrera.

2.4 Primer potencial de ionización $E_{I}$, segundo potencial de ionización $E_{I I} \mathrm{y}$ energía $E=E_{I}+E_{I I}$ del estado base del átomo de helio confinado, como función del radio de confinamiento y la altura de la barrera.

2.5 Radios críticos de la primera y segunda ionización del átomo de helio confinado, para diferentes alturas de la barrera.

2.6 Entropía informática de Shannon del átomo de helio y el ion $\mathrm{He}^{+}$, confinados dentro de una barrera esférica de radio $R_{c}$ y altura $V_{0}$

2.7 Corrección de núcleo de tamaño finito a la energía del estado base del átomo de helio confinado dentro de una esfera penetrable, como función del radio de confinamiento y la altura de la barrera

3.1 Cálculo variacional de la energía del estado $1 s^{2} 2 s$ del litio confinado dentro de una cavidad esférica impenetrable, como función del radio de confinamiento

3.2 Parámetros variacionales $\alpha, \beta, \gamma$ y energía del estado $1 s^{2} 2 p_{z}$ del átomo de litio confinado, como función del radio de confinamiento.

3.3 Corrección de núcleo finito del estado $1 s^{2} 2 s$ del átomo de litio confinado para un núcleo de ${ }^{7} L i\left(r_{0}=2.39 \mathrm{fm}\right)$ y un núcleo de ${ }^{6} L i\left(r_{0}=2.55 \mathrm{fm}\right)$, como función del radio de confinamiento.

3.4 Corrección de núcleo finito del estado $1 s^{2} 2 p_{z}$ del átomo de litio confinado para un núcleo de ${ }^{7} L i\left(r_{0}=2.39 \mathrm{fm}\right)$ y un núcleo de ${ }^{6} L i\left(r_{0}=2.55 \mathrm{fm}\right)$, como función del radio de confinamiento. 
4.1 Energía del estado base y corrección de núcleo finito, como función del radio de confinamiento, para un átomo muónico confinado con núcleo de

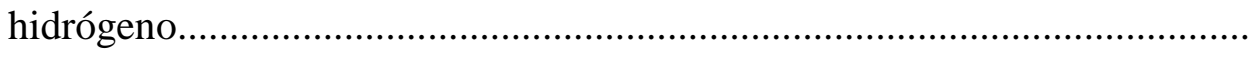

4.2 Energía del estado base y corrección de núcleo finito, como función del radio de confinamiento, para un átomo muónico confinado con núcleo de ${ }^{4} \mathrm{He}$

4.3 Energía del estado base y corrección de núcleo finito, como función del radio de confinamiento, para un átomo muónico confinado con núcleo de ${ }^{6} \mathrm{Li}$

4.4 Energía del estado base y corrección de núcleo finito, como función del radio de confinamiento, para un átomo muónico confinado con núcleo de ${ }^{7} \mathrm{Li}$ 


\section{Introducción}

Cuando los electrones de un material se encuentran restringidos a moverse en una región pequeña del espacio, se dice que el sistema está confinado espacialmente. Un sistema cuántico confinado se caracteriza por tener una función de estado que satisface determinadas condiciones de contorno para valores finitos de las coordenadas. El estudio de estos sistemas empezó a tomar importancia en la década de los años treinta cuando Michels, de Boer y Bijl [1] estudiaron la variación de la polarizabilidad de un átomo de hidrógeno sometido a altas presiones externas. El modelo propuesto por Michels et al. para dicho estudio consistió en un átomo de hidrógeno confinado en el centro de una caja esférica impenetrable. Desde entonces, el confinamiento dentro de cajas de diferentes tamaños y formas geométricas se ha vuelto muy popular y utilizado en una amplia variedad de sistemas cuánticos, para estudiar su comportamiento bajo presiones extremadamente altas o cuando se encuentran dentro de un sólido [1-78].

En primera aproximación, el comportamiento de un sistema (como puede ser un átomo, molécula, etc.) sujeto a presiones externas, puede ser simulado colocándolo dentro de una caja de paredes impenetrables, donde el potencial infinito es inducido por las partículas vecinas de carga negativa [2]. Bajo estas condiciones, la función de onda de la partícula debe hacerse cero en las paredes, satisfaciendo las condiciones de frontera de Dirichlet. Sin embargo, este modelo sólo incluye efectos producidos por fuerzas repulsivas. Para tomar en cuenta las fuerzas de atracción entre partículas, tales como las fuerzas de van der Waals, se ha propuesto que el pozo de potencial de confinamiento sea finito (es decir, una caja de paredes penetrables). Gracias a este modelo de confinamiento mediante paredes penetrables, por ejemplo, se ha podido reproducir el valor experimental del desdoblamiento hiperfino de un átomo de hidrógeno dentro de cuarzo $\alpha$ [31]. 
El confinamiento espacial induce cambios en las propiedades observables de los sistemas, tales como el espectro de energías, frecuencias y probabilidades de transición, polarizabilidad, etc. [1-78] Esta misma situación se ha encontrado a escala nanoscópica en sistemas artificiales construidos dentro de semiconductores, tales como pozos cuánticos en dos dimensiones, alambres y puntos cuánticos [3-8]. El confinamiento por paredes repulsivas también se encuentra en átomos sometidos a presiones extremas, átomos dentro de cavidades (como las zeolitas), en las burbujas de helio que se forman en los muros de los reactores nucleares bajo el bombardeo de partículas $\alpha$, etc. [9]

Los efectos causados por el confinamiento espacial, a su vez, han llevado a que los sistemas cuánticos confinados tengan una amplia variedad de aplicaciones científicas y tecnológicas, por ejemplo: han ayudado a comprender los efectos sobre la estructura electrónica en los átomos y moléculas que se encuentran atrapados en fullerenos [10] y en algunas cavidades microscópicas. En el estudio del calor específico en metales [11], datos espectroscópicos de astrofísica [12], materia atrapada dentro de campos electromagnéticos [13], modelos nucleares [14], etc. Algunos autores han sugerido el uso de espines aislados de átomos confinados dentro de jaulas de fullerenos para adaptarse como q-bits para computadoras cuánticas $[9,15,16]$.

El modelo de confinamiento ha sido ampliamente utilizado para estudiar el átomo de hidrógeno dentro de cavidades esféricas tanto de paredes duras como suaves [1, 2, 17-49], y con cajas de confinamiento de diferentes formas geométricas [50-55]. También se extiende su aplicación al estudio del átomo de helio [17, 26, 29, 56-64, 74], átomos multielectrónicos [65-71], moléculas [72, 73] e incluso del oscilador armónico [11, 14, 36].

A pesar de la gran variedad de trabajos dedicados al estudio de sistemas cuánticos confinados, algunas de sus características y efectos que ocurren dentro de éstos han sido poco estudiadas, como es el caso de los efectos relativistas debidos al movimiento de los electrones. Cuando el tamaño del confinamiento disminuye, los electrones alrededor del núcleo se mueven en un espacio cada vez más reducido, provocando un correspondiente incremento en la energía cinética, de acuerdo con el Principio de Incertidumbre de Heisenberg. Por tanto, es de esperar que la corrección relativista a la energía del átomo confinado sea mayor que en el caso del átomo libre. En sistemas confinados espacialmente, los efectos relativistas han sido 
estudiados directamente usando la ecuación de Dirac [75, 76], y para el estudio de excitones confinados en pozos y puntos cuánticos $[77,78]$. Parte del desarrollo de este trabajo estará dedicado al estudio del espectro de estructura fina del átomo de hidrógeno confinado y a establecer si los efectos relativistas en dicho sistema son lo suficientemente pequeños como para ser considerados como perturbaciones.

En la mayoría de los trabajos relacionados con átomos y moléculas, se asume que los núcleos son partículas puntuales, con carga y masa, pero sin extensión espacial. La inclusión de un núcleo de tamaño finito en los sistemas atómicos y moleculares, además de ser un modelo más realista, genera un corrimiento en la energía de los electrones [79-87]. La magnitud de este corrimiento es muy pequeña, comparada con la energía del sistema considerando un núcleo de tamaño puntual. Sin embargo, el estudio teórico y experimental de este efecto de volumen del núcleo es importante, ya que puede proveer información acerca de la estructura interna de éste. Además, algunos efectos considerados por la electrodinámica cuántica dependen de la distribución de la densidad de carga nuclear, como la polarización del vacío y la auto-energía [88]. En cálculos relativistas es muy útil el cambio a un modelo de núcleo de tamaño finito, ya que remueve la singularidad en el origen provista por el potencial coulombiano y permite la solución analítica de la ecuación de Dirac [89-91].

Hasta la fecha, a excepción del trabajo de Goldman y Joslin [12], no se conocen estudios publicados sobre átomos confinados en los que se incluya la corrección a la energía de los estados propios por el hecho de que el núcleo no es puntual.

Tampoco hay estudios sobre átomos exóticos confinados, como lo son los átomos muónicos. El muón negativo $\mu^{-}$es una partícula la cual tiene las mismas propiedades de un electrón, excepto que su masa es aproximadamente 207 veces mayor a la de éste. En particular, no es sensible a interacciones fuertes, por lo que su interacción con el núcleo atómico es meramente electromagnético.

Supongamos que un haz de muones es enviado a un objetivo de cierto material. Un muón negativo puede ser atraído por el campo electromagnético de un núcleo atómico, formando un estado ligado con éste. El sistema constituido de esta forma es a lo que se le conoce como átomo muónico. 
Dada la mayor masa del muón comparada a la del electrón, y sabiendo que el radio de Bohr es inversamente proporcional a la masa de la partícula, resulta que el radio de la órbita más baja de un muón es mucho menor que el radio orbital de los electrones que giran alrededor del núcleo, por lo que prácticamente la carga nuclear es la única con la que interactúa el muón [79]. Esto además hace que la influencia de la distribución espacial de la carga nuclear sobre los niveles de energía sea mayor que en el caso de los átomos convencionales [92, 93]. Si a esto se le suma el efecto de confinamiento espacial, se puede esperar un corrimiento mayor en las energías del sistema. Las mediciones de estos corrimientos en la energía ofrecen información detallada sobre la distribución de carga espacial y la distribución de la masa dentro del núcleo, y su dependencia con el espín nuclear.

Desafortunadamente, el muón es una partícula inestable (su vida media es de $2.2 \mu \mathrm{s}$ ), por lo que un átomo exótico sólo existe por un corto período de tiempo. Esto hace que su caracterización espectroscópica sea una tarea difícil. Sin embargo, el avance de la tecnología en años recientes ha hecho posible la producción de átomos exóticos suficientes para realizar mediciones espectroscópicas precisas de sus niveles de energía y sus probabilidades de transición [94].

En años recientes, la Teoría de la Información aplicada a sistemas atómicos y moleculares ha captado mucho la atención por parte de científicos e investigadores. Específicamente, la entropía informática de Shannon, cuyo concepto fue introducido por Claude E. Shannon [95], la cual es una medida de la cantidad de información perdida cuando el valor de una variable aleatoria es desconocido, se ha utilizado en sistemas atómicos y moleculares como una medida de la calidad de la función de onda $[96,97]$. En sistemas cuánticos confinados es una propiedad útil que se utiliza como una medida de la localización o deslocalización de la partícula $[42,43]$. En diversos estudios se ha observado que el comportamiento de la entropía de Shannon en el espacio de coordenadas está relacionada con la entropía de Shannon en el espacio de momentos de tal manera que guarda cierta similitud con el Principio de Incertidumbre de Heisenberg [42, 96, 99].

La entropía de Fisher es una medida de la concentración de la densidad de probabilidad, por lo que muchas veces se utiliza como complemento de la entropía de Shannon, y en conjunto 
ambas se han usado, entre otras cosas, para medir la correlación electrónica en sistemas de muchos electrones [100].

\section{Objetivos}

El objetivo general de este trabajo de tesis es estudiar, desde un punto de vista cuántico, los efectos de confinamiento dentro de cavidades esféricas impenetrables y penetrables en función del radio de confinamiento, en los siguientes contextos:

1. El efecto de diferentes funciones de corte sobre la energía del hidrógeno confinado cuando se usa el método variacional. La calidad de la función de onda se determina calculando la entropía informática de Shannon.

2. La estructura electrónica de átomos de uno, dos y tres electrones, y átomos muónicos, y la evolución de las energías para estados de diferentes simetrías, así como también analizar los cambios en la densidad de probabilidad electrónica y la presión sobre las paredes de una cavidad esférica penetrable.

3. El efecto que tiene el considerar un núcleo extendido, en lugar de uno puntual, en las energías de átomos de uno a tres electrones y en átomos muónicos confinados.

\section{Organización de este trabajo}

Esta tesis está organizada de la siguiente manera:

En el capítulo primero se estudia al átomo de hidrógeno confinado, dentro de una cavidad esférica. En la primera parte del capítulo se usa el método variacional directo o de RayleighRitz para hallar la energía del estado base del sistema como función del radio de confinamiento y de la altura de la barrera. En el caso de barreras impenetrables, se estudia el papel de la función de corte y se utiliza a la entropía informática de Shannon como medida de la calidad de la función de onda. En el caso de barreras penetrables se estudia la variación de algunas propiedades físicas del sistema, por ejemplo la polarizabilidad y la presión, como función de la penetrabilidad de la caja.

En la segunda parte del capítulo se resuelve el mismo problema pero en forma exacta, y se comparan los eigenvalores de la energía obtenidos con los reportados en la literatura. El espectro de energías y las funciones de onda así logrados son de utilidad para calcular, por 
medio de aproximaciones, otras propiedades físicas del sistema como el espectro de estructura fina y la corrección a la energía del estado base debida al tamaño finito del núcleo.

En el capítulo segundo se estudian algunas propiedades observables del átomo de helio confinado dentro de barreras esféricas tanto impenetrables como penetrables. Se usa el método variacional directo para su solución. Entre las propiedades estudiadas está la corrección a la energía del estado base debida al núcleo de tamaño finito, y el radio crítico de ionización usando el método de superposición. En el tercer capítulo se analiza la corrección de núcleo de tamaño finito, para el caso del átomo de litio confinado dentro de una cavidad esférica de paredes impenetrables.

En el cuarto capítulo se estudia el espectro de energías del átomo muónico atrapado dentro de una cavidad esférica de paredes impenetrables, como función del radio de confinamiento. Como se mencionó anteriormente, el efecto de la distribución finita de la carga nuclear es importante en este sistema, por lo que se analiza cómo varía el espectro de energías en función del tamaño del núcleo.

Finalmente, se presentan las conclusiones generales obtenidas en la realización de este trabajo y las perspectivas para trabajos futuros. 


\section{Capítulo 1}

\section{Átomo de hidrógeno confinado}

\subsection{Solución por el método variacional de Rayleigh-Ritz}

El método variacional de Rayleigh-Ritz es una técnica utilizada para hallar soluciones de la ecuación de Schrödinger, basada en la optimización del funcional de la energía.

Supongamos que se desea calcular la energía del estado base $E_{0}$ de un sistema descrito por el hamiltoniano $H$, pero no somos capaces de resolver de forma analítica la ecuación de Schrödinger. Entonces se hace uso del teorema variacional:

Teorema: Sea $E_{0}$ el eigenvalor correspondiente al estado base del sistema, entonces para cualquier función de prueba $\psi_{t}$ que cumpla con las constricciones del sistema se satisface la siguiente desigualdad

$$
\frac{\left\langle\psi_{t}|H| \psi_{t}\right\rangle}{\left\langle\psi_{t} \mid \psi_{t}\right\rangle} \geq E_{0}
$$

La igualdad se cumple cuando la función de prueba es igual a la función de onda exacta del estado base, $\psi_{t}=\psi_{0}$. La demostración de este teorema se encuentra en diversos libros de texto $[101,102]$.

En la práctica, $\psi_{t}$ depende de uno o más parámetros, y variándolos se puede encontrar el valor mínimo de la energía.

El Principio Variacional es extremadamente poderoso. El problema con este método es que muchas veces no se puede saber qué tan cerca se está del valor exacto de la energía del estado base (lo único de lo que se puede estar seguro es de que se obtiene una cota superior). 


\subsubsection{AHC dentro de paredes impenetrables}

El átomo de hidrógeno confinado (AHC) dentro de una esfera de paredes impenetrables es un modelo muy utilizado para probar nuevas técnicas de cálculo de eigenvalores o nuevas funciones de onda de prueba, ya que los resultados se pueden comparar con los cálculos exactos. Una de las técnicas más recurrentes para calcular la energía del estado base del sistema es el método variacional de Rayleigh-Ritz, en el cual la función de onda de prueba es modificada, esto es, multiplicada por un factor de corte apropiado, para que se cumplan adecuadamente las condiciones de frontera de Dirichlet (la función de onda debe hacerse cero en $r=R_{c}$, donde $R_{c}$ es el radio de la esfera impenetrable). En la literatura, muchos autores han usado diferentes formas de la función de corte: lineal [28, 30, 48], exponencial [57], de la forma $\left(1-r / R_{c}\right)^{n}$, donde $n$ es un número entero positivo [61], etc.

Sin embargo, hasta la fecha, no se ha realizado un estudio sistemático acerca del efecto de la función de corte, aplicada en el método variacional, sobre la energía del estado base del átomo de hidrógeno confinado. En otras palabras, ¿la función de corte afecta el valor de la energía? Y si ese es el caso, ¿qué función de corte lleva a la energía más baja?

Para responder a estas cuestiones, como fue propuesto por Gorecki y Byers Brown [25, 27], después implementado por Marín y Cruz [28], en este trabajo se propone una función de prueba $\psi_{t}$ para el estado base del átomo de hidrógeno confinado dentro de una barrera impenetrable en términos de la función de onda del átomo de hidrógeno libre de confinamiento $\psi_{\text {free }}$, multiplicada por una función de corte no singular $f_{\text {cut }}$, con la cual $\psi_{t}$ satisface las condiciones de frontera de Dirichlet del sistema $\left(f_{c u t}=0\right.$ si $r=R_{c}$, donde $R_{c}$ es el radio de confinamiento),

$$
\psi_{t}=\psi_{\text {free }} f_{\text {cut }}
$$

Como se mencionó en el párrafo anterior, $\psi_{\text {free }}$ se toma como la función de onda del átomo de hidrógeno libre de confinamiento, que para el estado base está dada por [28, 48, 101, 102]

$$
\psi_{\text {free }}(r)=A e^{-\alpha r}
$$

Donde $A$ es una constante de normalización y $\alpha$ es el parámetro variacional, de manera que la función de prueba estará dada por 


$$
\psi_{t}=A e^{-\alpha r} f_{c u t}
$$

Con estas funciones de onda se procede a minimizar el funcional de la energía del sistema con respecto a $\alpha$, con la finalidad de obtener un límite superior para la energía del estado base $E_{10}$ del átomo de hidrógeno confinado, esto es, como se describe en la Sección 1.1:

$$
\frac{\left\langle\psi_{t}|H| \psi_{t}\right\rangle}{\left\langle\psi_{t} \mid \psi_{t}\right\rangle} \geq E_{10}
$$

Donde $H$ es el hamiltoniano del sistema confinado, que en unidades atómicas está dado por

$$
H=-\frac{1}{2} \nabla^{2}-\frac{1}{r}+V_{c}(r)
$$

Donde el primer término de la derecha es la energía cinética del electrón, el segundo término es el potencial de interacción coulombiana entre el protón y el electrón (se considera al núcleo del átomo fijo en el centro de la cavidad), y $V_{c}$ es el potencial de confinamiento

$$
V_{c}(r)=\left\{\begin{array}{cc}
0, & r \leq R_{c} \\
\infty, & r>R_{c}
\end{array}\right.
$$

El objetivo de esta sección es averiguar si la precisión en los cálculos de la energía del estado base del AHC usando el método variacional de Rayleigh-Ritz depende de la función de corte utilizada. Las funciones de corte que se compararon fueron las siguientes

$$
\begin{gathered}
f_{\text {cut }}(r)=j_{0}\left(\frac{X_{10}}{R_{c}} r\right) ; c_{1} j_{0}\left(\frac{X_{10}}{R_{c}} r\right)+c_{2} j_{0}\left(\frac{X_{20}}{R_{c}} r\right) ;\left(1-\frac{r}{R_{c}}\right)^{2} ;\left(1-\frac{r}{R_{c}}\right) e^{\frac{r}{R_{c}}} ; \\
1-\frac{r}{R_{c}} ; 1-\left(\frac{r}{R_{c}}\right)^{2} ; 1-\left(\frac{r}{R_{c}}\right)^{3} ; 1-\left(\frac{r}{R_{c}}\right)^{4}
\end{gathered}
$$

Donde $j_{0}$ es la función esférica de Bessel de orden cero. $X_{10}$ y $X_{20}$ son la primera y segunda raíces de la función esférica de Bessel de orden cero, respectivamente. $c_{1}$ y $c_{2}$ son parámetros que deben ser determinados.

Por tanto, las integrales que se necesitan calcular son (debido a la simetría del problema y la ortonormalidad de los armónicos esféricos, sólo hay que enfocarse en las integrales radiales):

$$
\left\langle\psi_{t}|H| \psi_{t}\right\rangle=\left\langle\psi_{t}|T| \psi_{t}\right\rangle+\left\langle\psi_{t}|V| \psi_{t}\right\rangle
$$

Donde el valor esperado de la energía potencial está dado por 


$$
\left\langle\psi_{t}|V| \psi_{t}\right\rangle=-\int \psi_{t} \frac{1}{r} \psi_{t} d^{3} \vec{r}=-\int_{0}^{R_{c}}\left[e^{-\alpha r} f_{c u t}(r)\right]^{2} r d r
$$

Para el término de la energía cinética del sistema se tiene

$$
\begin{aligned}
\left\langle\psi_{t}|T| \psi_{t}\right\rangle & =-\frac{1}{2} \int \psi_{t}\left(\nabla^{2} \psi_{t}\right) d^{3} \vec{r} \\
& =-\frac{1}{2} \int_{0}^{R_{c}} e^{-\alpha r} f_{\text {cut }}(r)\left\{\frac{1}{r^{2}} \frac{d}{d r}\left[r^{2} \frac{d}{d r}\left(e^{-\alpha r} f_{\text {cut }}(r)\right)\right]\right\} r^{2} d r
\end{aligned}
$$

Integrando por partes

$$
\left\langle\psi_{t}|T| \psi_{t}\right\rangle=\frac{1}{2} \int_{0}^{R_{c}}\left[\frac{d}{d r}\left(e^{-\alpha r} f_{\text {cut }}(r)\right)\right]^{2} r^{2} d r
$$

La integral de traslape está dada por

$$
\left\langle\psi_{t} \mid \psi_{t}\right\rangle=\int_{0}^{R_{c}}\left(e^{-\alpha r} f_{c u t}(r)\right)^{2} r^{2} d r
$$

De esta forma, resolviendo las integrales (1.10), (1.12) y (1.13) se obtiene el funcional de la energía del estado base del sistema, como función del parámetro variacional $\alpha$ y el radio de confinamiento $R_{c}$

$$
E_{v a r}\left(\alpha, R_{c}\right)=\frac{\left\langle\psi_{t}|H| \psi_{t}\right\rangle}{\left\langle\psi_{t} \mid \psi_{t}\right\rangle}=\frac{\left\langle\psi_{t}|T| \psi_{t}\right\rangle+\left\langle\psi_{t}|V| \psi_{t}\right\rangle}{\left\langle\psi_{t} \mid \psi_{t}\right\rangle}
$$

Minimizando $E_{\text {var }}$ respecto a $\alpha$ para un valor fijo de $R_{c}$, se halla una cota superior para la energía del estado base $E_{10}$ del átomo de hidrógeno confinado.

\subsubsection{Casos donde la función de corte incluye funciones esféricas de Bessel}

En el caso en el que $f_{c u t}(r)=j_{0}\left(X_{10} r / R_{c}\right)$, es necesario simplificar la integral (1.12) para acelerar los cálculos computacionales. Esto se logra usando la siguiente relación de recurrencia de las funciones esféricas de Bessel [103]

$$
(2 n+1) \frac{d}{d u} j_{n}(u)=n j_{n-1}(u)-(n+1) j_{n+1}(u)
$$

Entonces 


$$
\frac{d}{d r} j_{0}\left(\frac{X_{10}}{R_{c}} r\right)=-\frac{X_{10}}{R_{c}} j_{1}\left(\frac{X_{10}}{R_{c}} r\right)
$$

Por tanto, en la integral del valor esperado de la energía cinética (1.12) se tiene

$$
\left\langle\psi_{t}|T| \psi_{t}\right\rangle=\frac{1}{2} \int_{0}^{R_{C}}\left[-\alpha e^{-\alpha r} j_{0}\left(\frac{X_{10}}{R_{c}} r\right)-e^{-\alpha r} \frac{X_{10}}{R_{c}} j_{1}\left(\frac{X_{10}}{R_{c}} r\right)\right]^{2} r^{2} d r
$$

Esta integral es más sencilla de resolver computacionalmente que intentar resolver directamente la integral (1.12). La integral del valor esperado de la energía potencial y la integral de traslape no requieren simplificaciones, y pueden resolverse directamente como se muestran en (1.10) y (1.13).

Para el caso $f_{c u t}=c_{1} j_{0}\left(X_{10} r / R_{c}\right)+c_{2} j_{0}\left(X_{20} r / R_{c}\right)$, la función de onda de prueba es

$$
\psi_{t}=c_{1} j_{0}\left(\frac{X_{10}}{R_{C}} r\right) e^{-\alpha r}+c_{2} j_{0}\left(\frac{X_{20}}{R_{c}} r\right) e^{-\alpha r}
$$

Definimos las funciones $\phi_{i}$ como

$$
\phi_{i}=j_{0}\left(\frac{X_{i 0}}{R_{c}} r\right) e^{-\alpha r}, \quad i=1,2
$$

La energía es una función del parámetro variacional $\alpha$ y el radio de confinamiento $R_{c}, \mathrm{y}$ puede escribirse como [104]

$$
E_{v a r}\left(\alpha, R_{c}\right)=\frac{\left\langle\psi_{t}|H| \psi_{t}\right\rangle}{\left\langle\psi_{t} \mid \psi_{t}\right\rangle}=\frac{\sum_{i, j} c_{i} c_{j} h_{i j}}{\sum_{i, j} c_{i} c_{j} b_{i j}}
$$

Donde

$$
\begin{gathered}
h_{i j}=\left\langle\phi_{i}|H| \phi_{j}\right\rangle, \quad i, j=1,2 \\
b_{i j}=\left\langle\phi_{i} \mid \phi_{j}\right\rangle, \quad i, j=1,2
\end{gathered}
$$

Los elementos de matriz $h_{i j}$ están dados por

$$
h_{i j}=t_{i j}+v_{i j}
$$

Donde

$$
\begin{gathered}
v_{i j}=\left\langle\phi_{i}|V| \phi_{j}\right\rangle=-\int_{0}^{R_{c}} e^{-2 \alpha r} j_{0}\left(\frac{X_{i 0}}{R_{c}} r\right) j_{0}\left(\frac{X_{j 0}}{R_{c}} r\right) r d r \\
t_{i j}=\left\langle\phi_{i}|T| \phi_{j}\right\rangle=\frac{1}{2} \int_{0}^{R_{c}}\left(\frac{d}{d r} j_{0}\left(\frac{X_{i 0}}{R_{c}} r\right) e^{-\alpha r}\right)\left(\frac{d}{d r} j_{0}\left(\frac{X_{j 0}}{R_{c}} r\right) e^{-\alpha r}\right) r^{2} d r
\end{gathered}
$$


Esta última integral se puede desarrollar usando la relación de recurrencia (1.15), quedando como

$$
t_{i j}=\frac{1}{2} \int_{0}^{R_{C}} e^{-2 \alpha r}\left[\alpha j_{0}\left(\frac{X_{i 0}}{R_{C}} r\right)+\frac{X_{i 0}}{R_{c}} j_{1}\left(\frac{X_{i 0}}{R_{c}} r\right)\right]\left[\alpha j_{0}\left(\frac{X_{j 0}}{R_{c}} r\right)+\frac{X_{j 0}}{R_{C}} j_{1}\left(\frac{X_{j 0}}{R_{C}} r\right)\right] r^{2} d r
$$

Los elementos de la matriz de traslape están dados por

$$
b_{i j}=\int_{0}^{R_{c}} e^{-2 \alpha r} j_{0}\left(\frac{X_{i 0}}{R_{c}} r\right) j_{0}\left(\frac{X_{j 0}}{R_{c}} r\right) r^{2} d r
$$

Desafortunadamente, las integrales (1.24), (1.26) y (1.27) no pueden calcularse analíticamente, por lo que deben resolverse numéricamente. Para hacerlo, es necesario fijar $R_{c}$, dar un valor inicial de $\alpha$, resolver el problema de eigenvalores, luego cambiar el valor de $\alpha$ y nuevamente resolver el sistema, para obtener nuevos valores de $E_{v a r}, c_{1}$ y $c_{2}$. Este proceso se repite hasta obtener una gráfica como la que se muestra en la Figura 1.1.

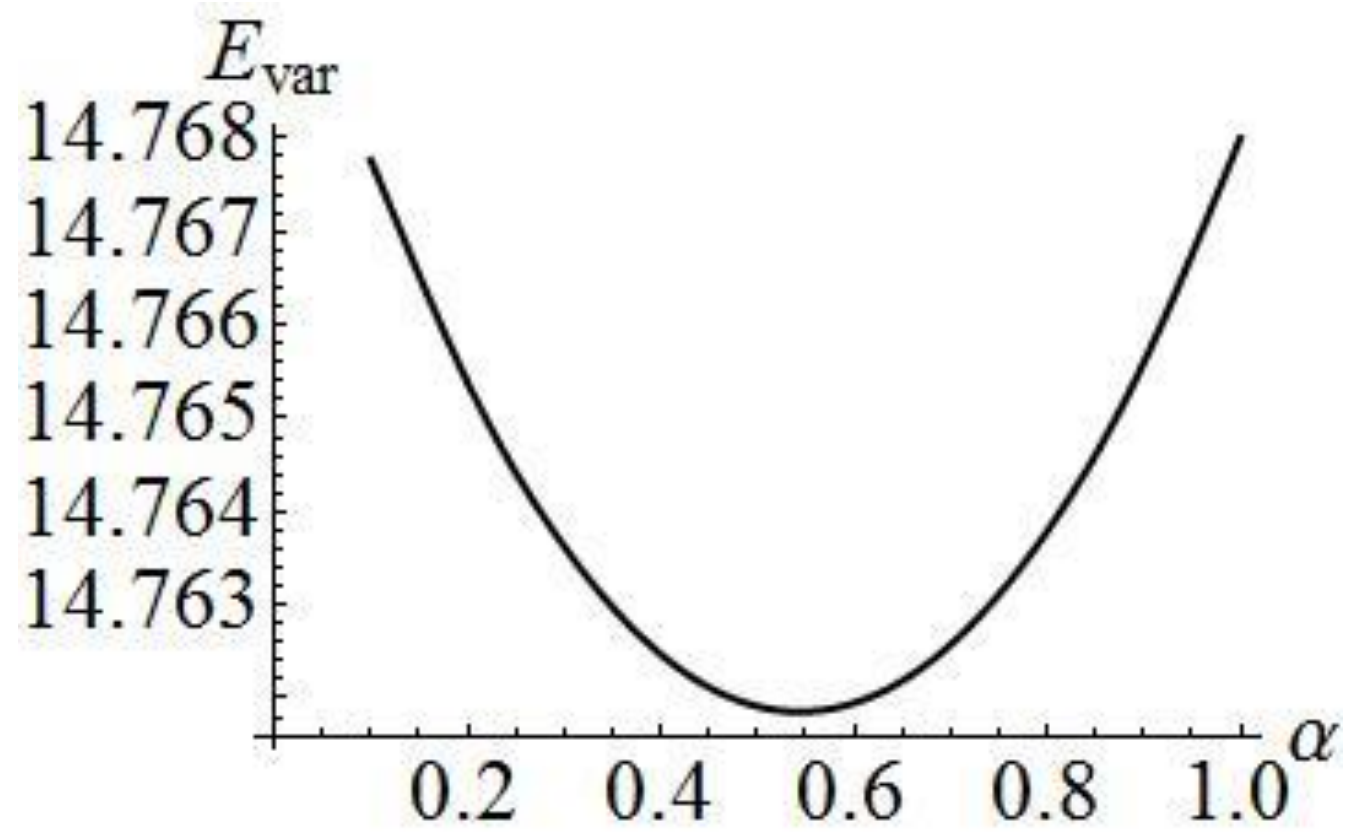

Figura 1.1. Energía del estado base del AHC como función del parámetro variacional $\alpha$. El radio de confinamiento de este ejemplo es $R_{c}=0.5$ bohrs. 
Los tres puntos con más baja energía se fijan a una curva parabólica usando el comando Efit de Mathematica. Minimizando esta ecuación cuadrática respecto a $\alpha$ se halla el valor óptimo del parámetro variacional. Con este valor optimizado se calculan los eigenvalores optimizados del hamiltoniano, así como los valores de los coeficientes $c_{1}$ y $c_{2}$.

\subsubsection{Entropía informática de Shannon como medida de la calidad de la función de prueba}

Como se mencionó en la Introducción, la entropía informática de Shannon se ha utilizado como una medida de la calidad del conjunto base en sistemas atómicos y moleculares libres de confinamiento [96, 97]. En esta sección se calculará la entropía de Shannon en el espacio de coordenadas del conjunto de funciones de prueba presentadas en la Sección 1.1.1., con el objetivo de analizar si la entropía de Shannon puede usarse como una medida de la calidad de la función de onda de prueba en el caso del átomo de hidrógeno confinado. De acuerdo al Principio de Máxima Entropía debido a Jaynes [98], la mejor función de onda es aquella que presente la más alta entropía de Shannon de un conjunto de funciones que satisfagan las constricciones del sistema. De esta manera, la entropía de Shannon podría ofrecer una forma alternativa de determinar la calidad de la función de prueba para sistemas confinados.

La entropía informática de Shannon en el espacio de coordenadas se define como

$$
S_{r}=-\int d^{3} \vec{r} \rho \ln \rho
$$

Donde $\rho \equiv\left|\psi_{t}\right|^{2}$ es la densidad de probabilidad del sistema. Para el átomo de hidrógeno confinado se calcula como

$$
S_{r}=-\int_{0}^{R_{c}} d^{3} \vec{r}\left|\psi_{t}\right|^{2} \ln \left|\psi_{t}\right|^{2}
$$

En esta última ecuación, de considera que $\psi_{t}$ está normalizada a la unidad. Sabiendo que $\psi_{t}=A e^{-\alpha r} f_{c u t}(r) Y_{00}(\theta, \varphi)$, donde $A$ es una constante de normalización y $Y_{00}(\theta, \varphi)=$ $1 / \sqrt{4 \pi}$, la ecuación (1.29) puede reescribirse como

$$
S_{r}=-2 \int_{0}^{R_{c}}\left[A e^{-\alpha r} f_{c u t}(r)\right]^{2} \ln \left[A e^{-\alpha r} f_{c u t}(r)\right] r^{2} d r-2 \ln \frac{1}{\sqrt{4 \pi}} .
$$


En las Tablas 1.1 y 1.2, se observa que para radios de confinamiento menores a 0.8 bohrs, la función de corte con la menor energía es $f_{c u t}(r)=j_{0}\left(X_{10} r / R_{c}\right)$, esto puede deberse a que en la región de confinamiento fuerte el sistema se comporta casi como una partícula libre dentro de una esfera de paredes impenetrables [45], cuyas funciones de onda radiales son precisamente funciones esféricas de Bessel [105]. Para radios de confinamiento entre 0.8 y 1.7 bohrs, la función de corte $1-\left(r / R_{c}\right)^{2}$ es con la que se obtienen las mejores energías.

Finalmente, para $R_{c}>2.0$ bohrs cualquier función de corte es buena para realizar cálculos, sin embargo la función de prueba $\left[1-\left(r / R_{c}\right)^{4}\right] e^{-\alpha r}$ es con la cual se obtiene la energía más baja. En estos casos el factor exponencial domina sobre la función de corte (para radios de confinamiento grandes, el sistema se comporta como un átomo de hidrógeno libre), además, cuando $R_{c} \rightarrow \infty, f_{\text {cut }} \rightarrow 1$ y se recuperan las funciones de onda del sistema libre de confinamiento.

Un caso especial ocurre con la función de prueba $\psi_{t}=\left(1-r / R_{c}\right) e^{-\alpha r}$, ya que cuando $R_{c}=$ 2.0 bohrs, se obtiene la función de onda exacta con $\alpha=0.5$. Las energías obtenidas usando esta función de onda son idénticas a las obtenidas usando la función $\psi_{t}=(1-$ $\left.r / R_{c}\right) e^{-\alpha r+r / R_{c}}$, y el parámetro variacional simplemente sufre un corrimiento $\alpha \rightarrow \alpha-$ $1 / R_{c}$.

Como se muestra en la Tabla 1.3, cuando se usa una función de corte compuesta por una combinación lineal de dos funciones esféricas de Bessel, se logra una ligera mejoría en la energía del estado base del sistema, en comparación con la función de prueba con una sola función esférica de Bessel. Es de esperar que entre más funciones esféricas de Bessel contenga la función de corte, será más baja la energía del estado base, y por tanto más cercana al valor real. Sin embargo, los cálculos computacionales se vuelven más lentos a medida que se agregan más funciones esféricas de Bessel a la función de corte.

El coeficiente $c_{1}$ domina, independientemente del radio de confinamiento, sin embargo para radios grandes el coeficiente $c_{2}$ se vuelve importante. 
Tabla 1.1. Energía del estado base del AHC, calculada usando diferentes funciones de corte $f_{\text {cut }}$ aplicadas en el método variacional. Se comparan los resultados con el valor exacto [35]. Las distancias están en bohrs y las energías en hartrees.

\begin{tabular}{|c|c|c|c|c|c|}
\hline$f_{c u t}$ & $1-r / R_{c}$ & $1-\left(r / R_{c}\right)^{2}$ & $1-\left(r / R_{c}\right)^{3}$ & $1-\left(r / R_{c}\right)^{4}$ & $\begin{array}{c}\text { Valor } \\
\text { Exacto } \\
{[35]}\end{array}$ \\
\hline 0.5 & 14.89705 & 14.81520 & 14.87737 & 15.03901 & 14.74797 \\
\hline 0.6 & 9.61804 & 9.56574 & 9.60564 & 9.71174 & 9.52771 \\
\hline 0.7 & 6.52721 & 6.49213 & 6.51900 & 6.59241 & 6.46993 \\
\hline 0.8 & 4.58076 & 4.55649 & 4.57516 & 4.62786 & 4.54338 \\
\hline 0.9 & 3.28700 & 3.26986 & 3.28309 & 3.32195 & 3.26219 \\
\hline 1.0 & 2.39058 & 2.37836 & 2.38782 & 2.41704 & 2.37399 \\
\hline 1.2 & 1.27666 & 1.27048 & 1.27527 & 1.29242 & 1.26931 \\
\hline 1.5 & 0.43883 & 0.43709 & 0.43840 & 0.44637 & 0.43702 \\
\hline 1.7 & 0.13960 & 0.13938 & 0.13947 & 0.14414 & 0.13910 \\
\hline 2.0 & -0.12500 & -0.12396 & -0.12491 & -0.12317 & -0.12500 \\
\hline 3.0 & -0.42254 & -0.42058 & -0.42241 & -0.42369 & -0.42397 \\
\hline 4.0 & -0.48109 & -0.47956 & -0.48111 & -0.48243 & -0.48326 \\
\hline 5.0 & -0.49467 & -0.49368 & -0.49476 & -0.49562 & -0.49642 \\
\hline 6.0 & -0.49821 & -0.49762 & -0.49832 & -0.49880 & -0.49928 \\
\hline 7.0 & -0.49929 & -0.49893 & -0.49937 & -0.49963 & -0.49986 \\
\hline 8.0 & -0.49967 & -0.49945 & -0.49973 & -0.49987 & -0.49997 \\
\hline 9.0 & -0.49983 & -0.49969 & -0.49987 & -0.49995 & -0.49999 \\
\hline 10.0 & -0.49990 & -0.49981 & -0.49993 & -0.49998 & -0.50000 \\
\hline
\end{tabular}


Tabla 1.2. Energía del estado base del AHC, calculada usando diferentes funciones de corte $f_{\text {cut }}$ aplicadas en el método variacional. Se comparan los resultados con el valor exacto [35]. Las distancias están en bohrs y las energías en hartrees.

\begin{tabular}{|c|c|c|c|c|}
\hline$f_{c u t}$ & $\left(1-r / R_{C}\right) e^{r / R_{C}}$ & $\left(1-r / R_{c}\right)^{2}$ & $j_{0}\left(X_{10} r / R_{C}\right)$ & $\begin{array}{c}\text { Valor } \\
\text { Exacto } \\
{[35]}\end{array}$ \\
\hline 0.5 & 14.89705 & 16.15122 & 14.76205 & 14.74797 \\
\hline 0.6 & 9.61804 & 10.49748 & 9.54187 & 9.52771 \\
\hline 0.7 & 6.52721 & 7.17906 & 6.48415 & 6.46993 \\
\hline 0.8 & 4.58076 & 5.08381 & 4.55767 & 4.54338 \\
\hline 0.9 & 3.28700 & 3.68728 & 3.27653 & 3.26219 \\
\hline 1.0 & 2.39058 & 2.71679 & 2.38838 & 2.37399 \\
\hline 1.2 & 1.27666 & 1.50531 & 1.28377 & 1.26931 \\
\hline 1.5 & 0.43883 & 0.58611 & 0.45148 & 0.43702 \\
\hline 1.7 & 0.13960 & 0.25414 & 0.15350 & 0.13910 \\
\hline 2.0 & -0.12500 & -0.04310 & -0.11080 & -0.12500 \\
\hline 3.0 & -0.42254 & -0.39022 & -0.41155 & -0.42397 \\
\hline 4.0 & -0.48109 & -0.46699 & -0.47406 & -0.48326 \\
\hline 5.0 & -0.49467 & -0.48835 & -0.49056 & -0.49642 \\
\hline 6.0 & -0.49821 & -0.49527 & -0.49588 & -0.49928 \\
\hline 7.0 & -0.49929 & -0.49783 & -0.49792 & -0.49986 \\
\hline 8.0 & -0.49967 & -0.49890 & -0.49884 & -0.49997 \\
\hline 9.0 & -0.49983 & -0.49939 & -0.49930 & -0.49999 \\
\hline 10.0 & -0.49990 & -0.49964 & -0.49955 & -0.50000 \\
\hline
\end{tabular}


Tabla 1.3. Energía del estado base del AHC calculada por el método variacional usando la función de prueba $\psi_{t}=\left[c_{1} j_{0}\left(X_{10} r / R_{c}\right)+c_{2} j_{0}\left(X_{20} r / R_{c}\right)\right] e^{-\alpha r}$. Se reportan los coeficientes $c_{1}$ y $c_{2}$. Las energías calculadas $E_{\text {var }}$ se comparan con el valor exacto [35].

\begin{tabular}{|c|c|c|c|c|}
\hline$R_{c}$ & $c_{1}$ & $c_{2}$ & $E_{\text {var }}$ & $\begin{array}{c}\text { Valor Exacto } \\
{[35]}\end{array}$ \\
\hline 0.5 & -0.99989 & 0.01466 & 14.76186 & 14.74797 \\
\hline 0.6 & -0.99983 & 0.01850 & 9.54163 & 9.52771 \\
\hline 0.7 & -0.99974 & 0.02257 & 6.48388 & 6.46993 \\
\hline 0.8 & -0.99964 & 0.02685 & 4.55736 & 4.54338 \\
\hline 0.9 & -0.99951 & 0.03133 & 3.27618 & 3.26219 \\
\hline 1.0 & -0.99935 & 0.03597 & 2.38798 & 2.37399 \\
\hline 1.2 & -0.99895 & 0.04580 & 1.28327 & 1.26931 \\
\hline 1.5 & -0.99809 & 0.06181 & 0.45082 & 0.43702 \\
\hline 1.7 & -0.99731 & 0.07326 & 0.15273 & 0.13910 \\
\hline 2.0 & -0.99580 & 0.09153 & -0.11176 & -0.12500 \\
\hline 3.0 & -0.98721 & 0.15943 & -0.41315 & -0.42397 \\
\hline 4.0 & -0.97372 & 0.22772 & -0.47603 & -0.48326 \\
\hline 5.0 & -0.95948 & 0.28179 & -0.49244 & -0.49642 \\
\hline 6.0 & -0.94905 & 0.31513 & -0.49739 & -0.49928 \\
\hline 7.0 & -0.94406 & 0.32976 & -0.49903 & -0.49986 \\
\hline 8.0 & -0.94345 & 0.33151 & -0.49962 & -0.49997 \\
\hline 9.0 & -0.94533 & 0.32612 & -0.49984 & -0.49999 \\
\hline 10.0 & -0.94808 & 0.31804 & -0.49993 & -0.50000 \\
\hline
\end{tabular}

Como puede verse en las Tablas 1.4 y 1.5, la entropía de Shannon en el espacio de coordenadas disminuye a medida que el radio de confinamiento disminuye, debido a que el sistema se encuentra más localizado. Sin embargo, contrario a lo que se esperaba, hay casos en los cuales la función de onda con la energía más baja no tiene la entropía de Shannon más alta. En las Figuras 1.2 y 1.3 se grafica la entropía de Shannon para cada función de prueba, y se comparan con la entropía de Shannon de la función de onda exacta. 
Tabla 1.4. Entropía de Shannon $S_{r}$ para cada función de prueba $\psi_{t}=A f_{c u t} e^{-\alpha r}$, como función del radio de confinamiento. Los radios están en bohrs.

\begin{tabular}{|c|c|c|c|c|}
\hline$f_{\text {cut }}$ & $1-r / R_{c}$ & $1-\left(r / R_{c}\right)^{2}$ & $1-\left(r / R_{c}\right)^{3}$ & $1-\left(r / R_{c}\right)^{4}$ \\
$R_{c}$ & & & & \\
\hline 0.5 & -1.45372 & -1.45981 & -1.45311 & -1.44413 \\
\hline 0.6 & -0.92247 & -0.92866 & -0.92186 & -0.91275 \\
\hline 0.7 & -0.47630 & -0.48262 & -0.47570 & -0.46644 \\
\hline 0.8 & -0.09260 & -0.09904 & -0.09200 & -0.08257 \\
\hline 0.9 & 0.24323 & 0.23665 & 0.24382 & 0.25343 \\
\hline 1.0 & 0.54115 & 0.53440 & 0.54172 & 0.55154 \\
\hline 1.2 & 1.04970 & 1.04259 & 1.05025 & 1.06053 \\
\hline 1.5 & 1.65591 & 1.64810 & 1.65639 & 1.66756 \\
\hline 1.7 & 1.98517 & 1.97678 & 1.98559 & 1.99749 \\
\hline 2.0 & 2.39667 & 2.38724 & 2.39700 & 2.41022 \\
\hline 3.0 & 3.29439 & 3.27996 & 3.29467 & 3.31412 \\
\hline 4.0 & 3.74216 & 3.72251 & 3.74350 & 3.76925 \\
\hline 5.0 & 3.94630 & 3.92494 & 3.94965 & 3.97688 \\
\hline 6.0 & 4.03601 & 4.01627 & 4.04102 & 4.06516 \\
\hline 7.0 & 4.07795 & 4.06096 & 4.08376 & 4.10340 \\
\hline 8.0 & 4.09977 & 4.08546 & 4.10575 & 4.12127 \\
\hline 9.0 & 4.11238 & 4.10031 & 4.11817 & 4.13040 \\
\hline 10.0 & 4.12030 & 4.11005 & 4.12575 & 4.13546 \\
\hline
\end{tabular}


Tabla 1.5. Entropía de Shannon $S_{r}$ para cada función de prueba $\psi_{t}=A f_{c u t} e^{-\alpha r}$, como función del radio de confinamiento. Los radios están en bohrs.

\begin{tabular}{|c|c|c|c|c|}
\hline$f_{\text {cut }}$ & $\left(1-r / R_{c}\right) e^{r / R_{c}}$ & $\left(1-r / R_{c}\right)^{2}$ & $j_{0}\left(X_{10} r / R_{c}\right)$ & $c_{1} j_{0}\left(X_{10} r / R_{c}\right)$ \\
$R_{c}$ & & & & \\
\hline 0.5 & -1.45372 & -1.58501 & -1.47629 & -1.47603 \\
\hline 0.6 & -0.92247 & -1.05472 & -0.94550 & -0.94519 \\
\hline 0.7 & -0.47630 & -0.60957 & -0.49986 & -0.49949 \\
\hline 0.8 & -0.09260 & -0.22693 & -0.11673 & -0.11630 \\
\hline 0.9 & 0.24323 & 0.10779 & 0.21847 & 0.21895 \\
\hline 1.0 & 0.54115 & 0.40455 & 0.51568 & 0.51621 \\
\hline 1.2 & 1.04970 & 0.91067 & 1.02262 & 1.02325 \\
\hline 1.5 & 1.65591 & 1.51303 & 1.62581 & 1.62659 \\
\hline 1.7 & 1.98517 & 1.83966 & 1.95261 & 1.95351 \\
\hline 2.0 & 2.39667 & 2.24738 & 2.35979 & 2.36090 \\
\hline 3.0 & 3.29439 & 3.13911 & 3.23568 & 3.24264 \\
\hline 4.0 & 3.74216 & 3.60146 & 3.67049 & 3.68557 \\
\hline 5.0 & 3.94630 & 3.83680 & 3.87382 & 3.90531 \\
\hline 6.0 & 4.03601 & 3.95735 & 3.97283 & 4.01764 \\
\hline 7.0 & 4.07795 & 4.02209 & 4.02591 & 4.07694 \\
\hline 8.0 & 4.09977 & 4.05925 & 4.05736 & 4.10834 \\
\hline 9.0 & 4.11238 & 4.08205 & 4.07757 & 4.12484 \\
\hline 10.0 & 4.12030 & 4.09690 & 4.09137 & 4.13354 \\
\hline
\end{tabular}




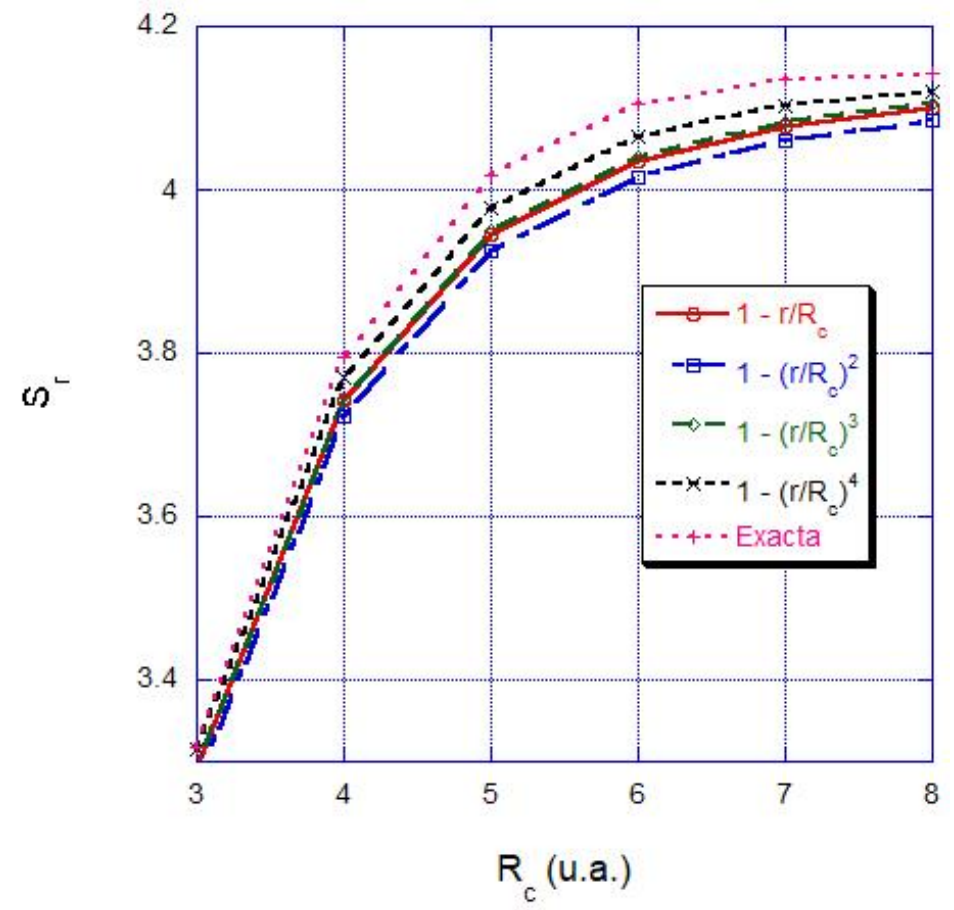

Figura 1.2. Entropía de Shannon $S_{r}$ como función del radio de confinamiento.

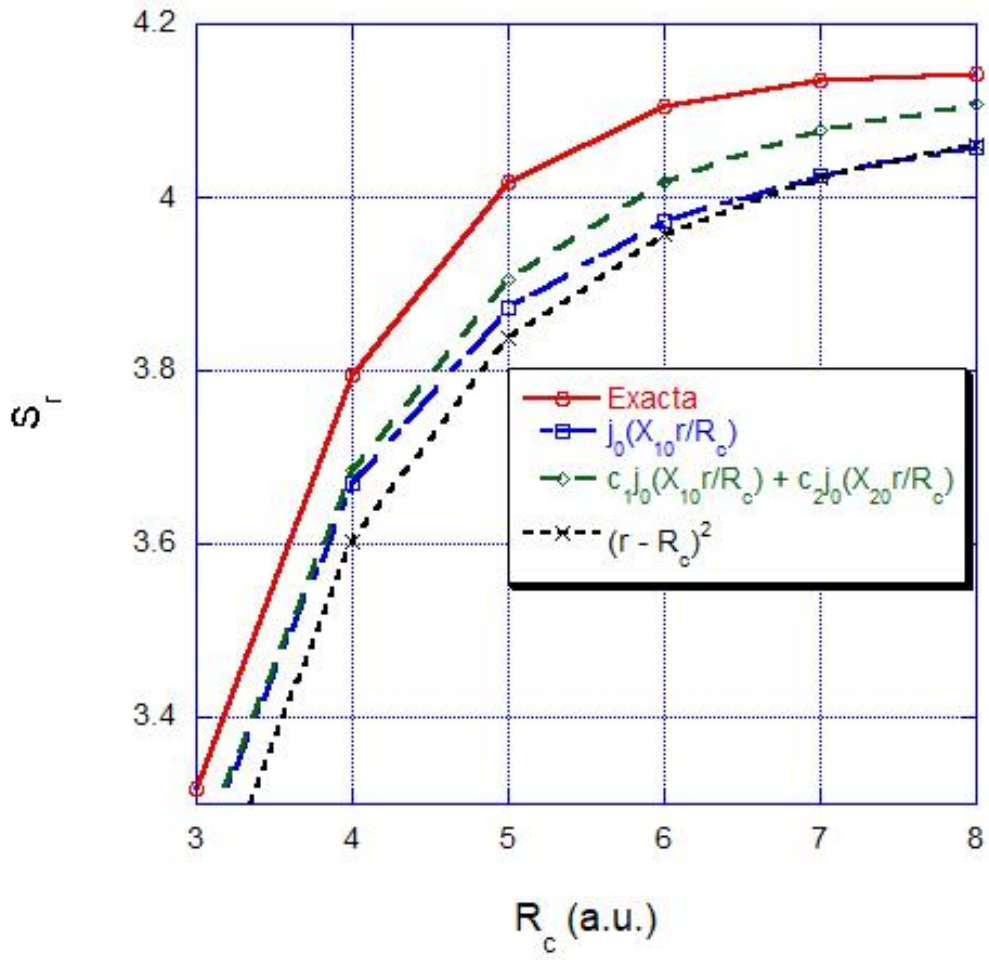

Figura 1.3. Entropía de Shannon $S_{r}$ como función del radio de confinamiento. 


\subsubsection{AHC dentro de paredes penetrables}

A pesar de que el modelo de paredes impenetrables es ampliamente utilizado por su relativa sencillez, sólo se pueden obtener resultados cualitativos, ya que éste sobreestima los efectos del confinamiento sobre las observables del sistema. Como un modelo más realista se ha propuesto el confinamiento dentro de paredes penetrables.

A finales de los años 70's, Ley-Koo y Rubinstein [31] utilizaron este modelo del átomo de hidrógeno confinado para explicar el resultado experimental para el desdoblamiento hiperfino del hidrógeno atómico en cuarzo- $\alpha$. Aunque encontraron soluciones analíticas para la ecuación de Schrödinger correspondiente, los eigenvalores de la energía fueron obtenidos resolviendo una ecuación trascendental. También calcularon el apantallamiento magnético nuclear, el término de contacto de Fermi, la polarizabilidad y la presión como funciones del radio de confinamiento y la altura de la barrera. Recientemente Montgomery y Sen [47] mejoraron los resultados obtenidos por Ley-Koo y Rubinstein, en tanto que autores como Marín y Cruz [29] implementaron el método variacional de Rayleigh-Ritz con una función de onda de prueba para el estado base construida a pedazos: una para la región interior $(r<$ $\left.R_{c}\right)$, y la otra para la región exterior $\left(r>R_{c}\right)$.

En esta sección se estudia el átomo de hidrógeno confinado dentro de una caja esférica de paredes penetrables usando el método variacional de Rayleigh-Ritz, con una nueva función de prueba que presenta una mayor estabilidad al momento de realizar cálculos computacionales. El hamiltoniano de este sistema (en unidades atómicas) está dado por

$$
H=-\frac{1}{2} \nabla^{2}+V_{c}
$$

El núcleo del sistema se encuentra fijo en el centro de la esfera. La energía potencial asociada a este sistema está dada por un término coulombiano dentro de la caja ( $0 \leq r \leq R_{c}$, donde $r$ es la distancia radial al centro de la esfera y $R_{c}$ el radio de confinamiento), y una barrera de altura constante $V_{0}$ fuera de la caja $\left(r>R_{c}\right)$ :

$$
V_{c}=\left\{\begin{array}{c}
-\frac{1}{r}, \quad 0 \leq r \leq R_{c} \\
V_{0}, r>R_{c}
\end{array}\right.
$$

Para estudiar el estado base del sistema se utiliza la siguiente función de prueba 


$$
\begin{gathered}
R_{i}(r)=C e^{-\alpha r} j_{0}\left(\frac{\gamma \pi r}{R_{c}}\right), \quad 0 \leq r \leq R_{c} \\
R_{e}(r)=B \frac{e^{-\beta r}}{r}, \quad r>R_{c}
\end{gathered}
$$

Donde $B$ y $C$ son constantes de normalización y $\alpha, \beta$ y $\gamma$ son parámetros variacionales. $j_{0}$ es la función esférica de Bessel de orden cero.

El factor $e^{-\alpha r}$ toma en cuenta la interacción coulombiana entre el electrón y el núcleo. Si $\alpha=0$, las ecuaciones (1.33) y (1.34) corresponden a la función de onda de una partícula libre dentro de una cavidad esférica penetrable $[105,106]$. Se eligió esta forma de la función de onda ya que, como se mostró en la Sección 1.1.1, para sistemas fuertemente confinados se obtienen buenos resultados para la energía del estado base.

La función de onda radial debe ser continua en todo el espacio, en particular en $r=R_{c}$, es decir

$$
R_{i}\left(R_{c}\right)=R_{e}\left(R_{c}\right)
$$

De donde se obtiene la relación entre las constantes de normalización

$$
B=C R_{c} j_{0}(\gamma \pi) e^{(\beta-\alpha) R_{c}}
$$

De la derivada logarítmica de la función de onda radial dentro de la caja se obtiene

$$
\frac{R_{i}^{\prime}\left(R_{c}\right)}{R_{i}\left(R_{c}\right)}=-\frac{\gamma \pi}{R_{c}} \frac{j_{1}(\gamma \pi)}{j_{0}(\gamma \pi)}-\alpha
$$

Mientras que para la sección fuera de la caja

$$
\frac{R_{e}^{\prime}\left(R_{c}\right)}{R_{e}\left(R_{c}\right)}=-\beta-\frac{1}{R_{c}}
$$

La derivada logarítmica debe ser continua en todo el espacio, en particular en la frontera $r=$ $R_{c}$, por lo que igualando (1.37) y (1.38) se encuentra que

$$
\beta=\alpha+\frac{\gamma \pi j_{1}(\gamma \pi)}{R_{c} j_{0}(\gamma \pi)}-\frac{1}{R_{c}}
$$

De esta forma sólo es necesario tomar en cuenta dos parámetros variacionales independientes: $\alpha$ y $\gamma$. Hay que considerar que para que la solución sea físicamente aceptable, la ecuación (1.34) debe tender a cero cuando $r \rightarrow \infty$, por lo que $\beta$ debe ser $>0$. 
El funcional de la energía en el método variacional, que debe minimizarse respecto a los parámetros variacionales $\alpha$ y $\gamma$, está dado por

$$
E(\alpha, \gamma)=\frac{\langle R|T| R\rangle+\left\langle R\left|V_{c}\right| R\right\rangle}{\langle R \mid R\rangle}
$$

Donde la integral de traslape es

$$
\langle R \mid R\rangle=\int_{0}^{R_{C}}\left|R_{i}\right|^{2} r^{2} d r+\int_{R_{c}}^{\infty}\left|R_{e}\right|^{2} r^{2} d r
$$

Demandando que esta ecuación sea igual a la unidad se encuentra el valor de $C$.

La integral de la energía cinética

$$
\begin{aligned}
\langle R|T| R\rangle=\int_{0}^{R_{C}} & R_{i}^{*}\left(-\frac{1}{2} \frac{1}{r^{2}} \frac{d}{d r}\left(r^{2} \frac{d}{d r} R_{i}\right)\right) r^{2} d r \\
& +\int_{R_{c}}^{\infty} R_{e}^{*}\left(-\frac{1}{2} \frac{1}{r^{2}} \frac{d}{d r}\left(r^{2} \frac{d}{d r} R_{e}\right)\right) r^{2} d r
\end{aligned}
$$

Y la integral de la energía potencial

$$
\left\langle R\left|V_{c}\right| R\right\rangle=\int_{0}^{R_{C}} R_{i}^{*}\left(-\frac{1}{r}\right) R_{i} r^{2} d r+\int_{R_{C}}^{\infty} R_{e}^{*} V_{0} R_{e} r^{2} d r
$$

Los cálculos, algebraicos y numéricos, se realizaron con el programa Mathematica 9. Para obtener el valor mínimo de la energía se empleó la expresión (1.40) y el comando FindMinimum, dando valores iniciales para la iteración de $\alpha$ y $\gamma$ menores a 2. El programa Mathematica siempre encontró el valor mínimo de la energía. Cuando se repitió este procedimiento empleando las funciones de prueba de Marín y Cruz, el programa en ocasiones se detenía, y había que dar valores iniciales diferentes para $\alpha$ y $\gamma$ para poder determinar el valor mínimo de la energía. La función de prueba de este trabajo proporciona un funcional de la energía del cual se puede obtener el valor mínimo de la energía en forma automatizada. 


\subsubsection{Propiedades físicas}

Una vez obtenida la energía del estado base, así como los valores óptimos de los parámetros variacionales, podemos calcular algunas propiedades físicas. Para el átomo de hidrógeno confinado, algunas de las más importantes son:

1. El desdoblamiento hiperfino, dado por el término de contacto de Fermi [31, 33]

$$
A=(2 / 3) g \mathrm{~B}_{n} \mathrm{~B}_{n}|R(0)|^{2}
$$

2. El apantallamiento magnético nuclear [31, 33], dado por la constante de apantallamiento diamagnético

$$
\sigma=\frac{e^{2}}{3 \mu c^{2}}\left\langle r^{-1}\right\rangle
$$

3. La polarizabilidad en la aproximación de Buckingham [107]

$$
\alpha_{d}=\frac{2}{3}\left[\frac{6\left\langle r^{2}\right\rangle^{3}+3\left\langle r^{3}\right\rangle^{2}-8\langle r\rangle\left\langle r^{2}\right\rangle\left\langle r^{3}\right\rangle}{9\left\langle r^{2}\right\rangle-8\langle r\rangle^{2}}\right]
$$

4. La presión, calculada como

$$
P=-\frac{1}{4 \pi R_{c}^{2}} \frac{d E}{d R_{c}}
$$

5. La probabilidad de tunelamiento del electrón, dada por la segunda integral en la ecuación (1.41)

$$
p\left(r>R_{c}\right)=\int_{R_{C}}^{\infty}\left|R_{e}\right|^{2} r^{2} d r
$$

En la Tabla 1.6 se muestran los valores óptimos de los parámetros variacionales $\alpha$ y $\gamma$, y la energía del estado base $E_{10}$ del sistema, para diferentes radios de confinamiento y barreras de altura $V_{0}=0,2$ y 5 hartrees. Estos eigenvalores se comparan con los reportados por Marín y Cruz [29], quienes también utilizaron el método variacional directo, y con los reportados por Ley-Koo y Rubinstein [31], cuyos resultados son exactos hasta las cifras reportadas. Como puede observarse, existe una buena concordancia entre los eigenvalores obtenidos por el método variacional usando la función de prueba aquí propuesta y los resultados reportados por los otros autores. 
Los eigenvalores de la energía aumentan a medida que el radio de confinamiento decrece; sin embargo, para que el electrón permanezca en un estado ligado, la energía debe ser menor o igual a $V_{0}$. Esto da lugar al hecho de que, para un valor fijo de $V_{0}$, el electrón permanece en un estado ligado sólo para cajas cuyo radio es $R_{c}>R^{*}$, donde $R^{*}$ es el radio crítico para el cual $E=V_{0}$. Para $V_{0}=\infty$ la energía crece, aparentemente sin límite, a medida que $R_{c} \rightarrow 0$.

Para cajas de cierto radio, los eigenvalores de la energía son mayores para valores más grandes de $V_{0}$, esto es, para paredes menos penetrables. Conforme $R_{c}$ aumenta, los eigenvalores de la energía tienden asintóticamente al valor de la energía del estado base del átomo de hidrógeno libre de confinamiento.

Para la mayoría de radios de confinamiento y alturas de la barrera, las energías calculadas con la función de prueba usada en este trabajo son iguales o están ligeramente por arriba de las obtenidas por Marín y Cruz. Sólo para radios pequeños y alturas de la barrera grandes es que se obtienen energías más bajas. 
Tabla 1.6. Energía $E_{10}$ del estado base del AHC dentro de una caja penetrable de radio $R_{c}$ y altura de la barrera $V_{0}$. Las distancias están en bohrs y las energías en hartrees.

\begin{tabular}{|c|c|c|c|c|c|}
\hline$R_{c}$ & $\alpha$ & $\gamma$ & $E_{10}$ & Ref. [29] & Ref. [31] \\
\hline \multicolumn{5}{|c|}{$V_{0}=0$} \\
\hline 0.83155 & 0.9207 & 0.3504 & -0.0312 & -0.0313 & -0.0313 \\
\hline 1.00000 & 0.9027 & 0.4001 & -0.1249 & -0.1250 & -0.1250 \\
\hline 2.04918 & 0.8946 & 0.4967 & -0.4362 & -0.4366 & -0.4367 \\
\hline 4.08889 & 0.9714 & 0.3749 & -0.4978 & -0.4979 & -0.4980 \\
\hline 5.77827 & 0.9959 & 0.1884 & -0.4999 & -0.4999 & -0.4999 \\
\hline \multicolumn{7}{|c|}{$V_{0}=2$} \\
\hline 0.50746 & 0.8700 & 0.4306 & 1.9606 & 1.9607 & 1.9606 \\
\hline 0.59179 & 0.8388 & 0.4891 & 1.7149 & 1.7149 & 1.7147 \\
\hline 1.00791 & 0.7635 & 0.6382 & 0.5014 & 0.5003 & 0.5000 \\
\hline 4.90402 & 0.9296 & 0.6550 & -0.4966 & -0.4973 & -0.4980 \\
\hline 5.75669 & 0.9641 & 0.5410 & -0.4989 & -0.4992 & -0.4995 \\
\hline \multicolumn{7}{|c|}{$V_{0}=5$} & -596 & \\
\hline 0.42945 & 0.8202 & 0.5068 & 4.5915 & 4.5927 & 4.5914 \\
\hline 0.50502 & 0.7259 & 0.5638 & 3.8585 & 3.8593 & 3.8580 \\
\hline 1.55982 & 0.6858 & 0.8006 & -0.0195 & -0.0247 & -0.0247 \\
\hline 5.06334 & 0.9089 & 0.7534 & -0.4959 & -0.4971 & -0.4980 \\
\hline 5.49360 & 0.9307 & 0.7077 & -0.4975 & -0.4983 & -0.4990 \\
\hline
\end{tabular}

En la Tabla 1.7 se muestran los resultados obtenidos para las propiedades físicas presentadas en la sección anterior. En las Figuras 1.4 a 1.8 se muestra el comportamiento de dichas propiedades físicas como función del radio de confinamiento, para diferentes alturas de la barrera. 
Tabla 1.7. Término de contacto de Fermi $A$, constante de apantallamiento magnético $\sigma$, polarizabilidad $\alpha_{d}$, presión $P$ y probabilidad de tunelamiento $p\left(r>R_{c}\right)$ para el átomo de hidrógeno confinado en una esfera penetrable de radio $R_{c}$ y una barrera de altura $V_{0}$. Las energías están en hartrees y las distancias en bohrs.

\begin{tabular}{|c|c|c|c|c|c|}
\hline$R_{c}$ & $A^{\mathrm{a}}$ & $\sigma^{\mathrm{b}}$ & $\alpha_{d}{ }^{\mathrm{c}}$ & $P^{\mathrm{d}}$ & $p\left(r>R_{c}\right)$ \\
\hline \multicolumn{5}{|c|}{$V_{0}=0$} \\
\hline 1.0 & 76.45067 & 1.16253 & 0.96866 & 13.22021 & 0.57308 \\
\hline 2.0 & 61.89569 & 1.14581 & 0.30097 & 0.67250 & 0.12778 \\
\hline 3.0 & 52.46716 & 1.04889 & 0.44735 & 0.05595 & 0.03531 \\
\hline 4.0 & 50.63705 & 1.01410 & 0.57105 & 0.00616 & 0.00963 \\
\hline 5.0 & 50.56929 & 1.00354 & 0.63521 & 0.00075 & 0.00234 \\
\hline \multicolumn{5}{|c|}{$V_{0}=1$} \\
\hline 1.0 & 145.69089 & 1.65299 & 0.06731 & 32.49065 & 0.29414 \\
\hline 2.0 & 71.93924 & 1.27755 & 0.13111 & 1.30706 & 0.04761 \\
\hline 3.0 & 53.49968 & 1.10215 & 0.27760 & 0.12085 & 0.01185 \\
\hline 4.0 & 49.73204 & 1.03672 & 0.42873 & 0.01636 & 0.00359 \\
\hline 5.0 & 49.72637 & 1.01304 & 0.54622 & 0.00271 & 0.00116 \\
\hline \multicolumn{7}{|c|}{$V_{0}=5$} \\
\hline 1.0 & 233.12160 & 2.08924 & 0.01401 & 68.05642 & 0.08624 \\
\hline 2.0 & 79.84545 & 1.38067 & 0.07854 & 2.11443 & 0.01079 \\
\hline 3.0 & 53.37117 & 1.13869 & 0.20594 & 0.18893 & 0.00250 \\
\hline 4.0 & 48.06151 & 1.05152 & 0.34726 & 0.02631 & 0.00080 \\
\hline 5.0 & 48.12797 & 1.02077 & 0.46605 & 0.00488 & 0.00033 \\
\hline
\end{tabular}

${ }^{\mathrm{a}}$ En unidades $\frac{2}{3} g \mathrm{~B} g_{n} \mathrm{~B}_{n}=12.690565 \mathrm{mT}$, ben unidades $e^{2} /\left(3 \mu a_{0} c^{2}\right)$, cen unidades $10^{-24} \mathrm{~cm}^{3} \mathrm{y}$ den unidades $10^{6} \mathrm{~atm}$. 
Para valores fijos de $V_{0}$ y radios de confinamiento grandes, $A$ y $\sigma$ tienden asintóticamente a sus respectivos valores para el caso del átomo de hidrógeno libre de confinamiento ( $A=$ $50.762 \mathrm{mT}$ y $\left.\sigma=e^{2} / 3 \mu a_{0} c^{2}\right)$.Conforme el radio de confinamiento decrece, estas cantidades crecen monótonamente hasta alcanzar un valor máximo, y luego decrecen al aproximarse al radio crítico para el cual $E=V_{0}$. Para un radio de confinamiento fijo estas cantidades son más grandes para valores más altos de $V_{0}$. Para barreras impenetrables $\left(V_{0}=\right.$ $\infty), A$ y $\sigma$ crecen aparentemente sin límite a medida que $R_{c} \rightarrow 0$. Este comportamiento se muestra en las Figuras 1.4 y 1.5.

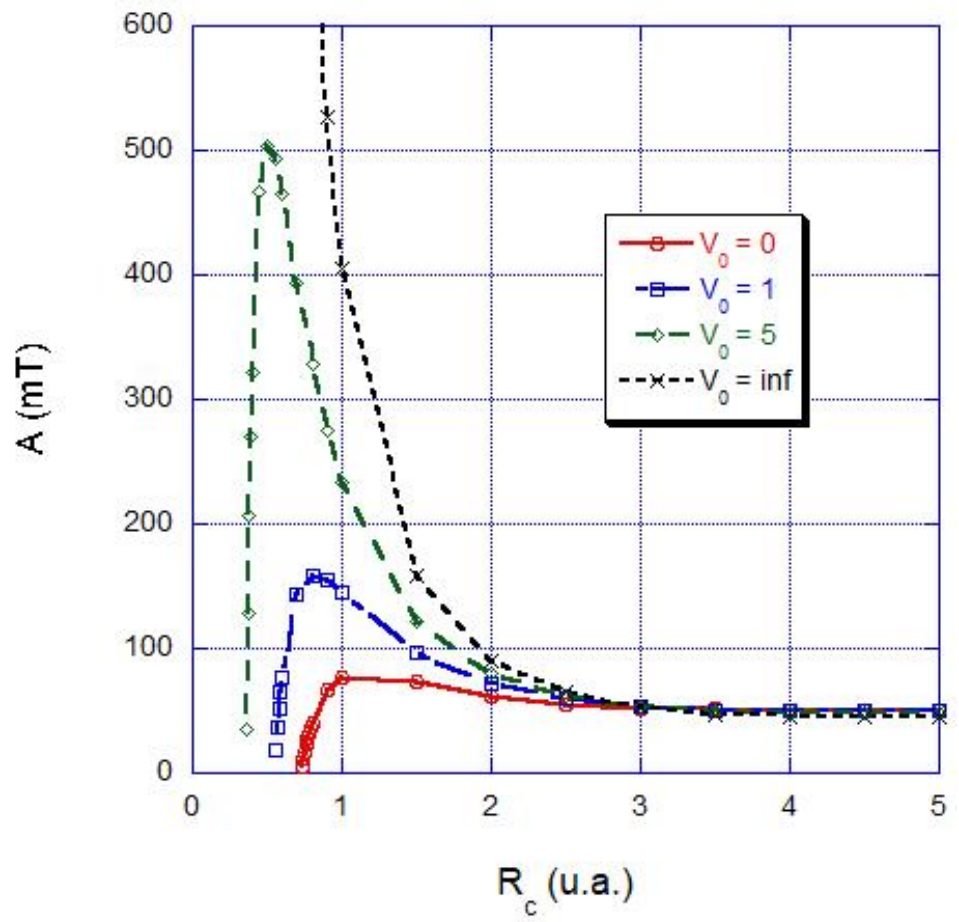

Figura 1.4. Constante de desdoblamiento hiperfino $A$ (en unidades $12.690565 \mathrm{mT}$ ) como función de $R_{c}$ (bohrs) para $V_{0}=0,1,5, \infty$ hartrees. 


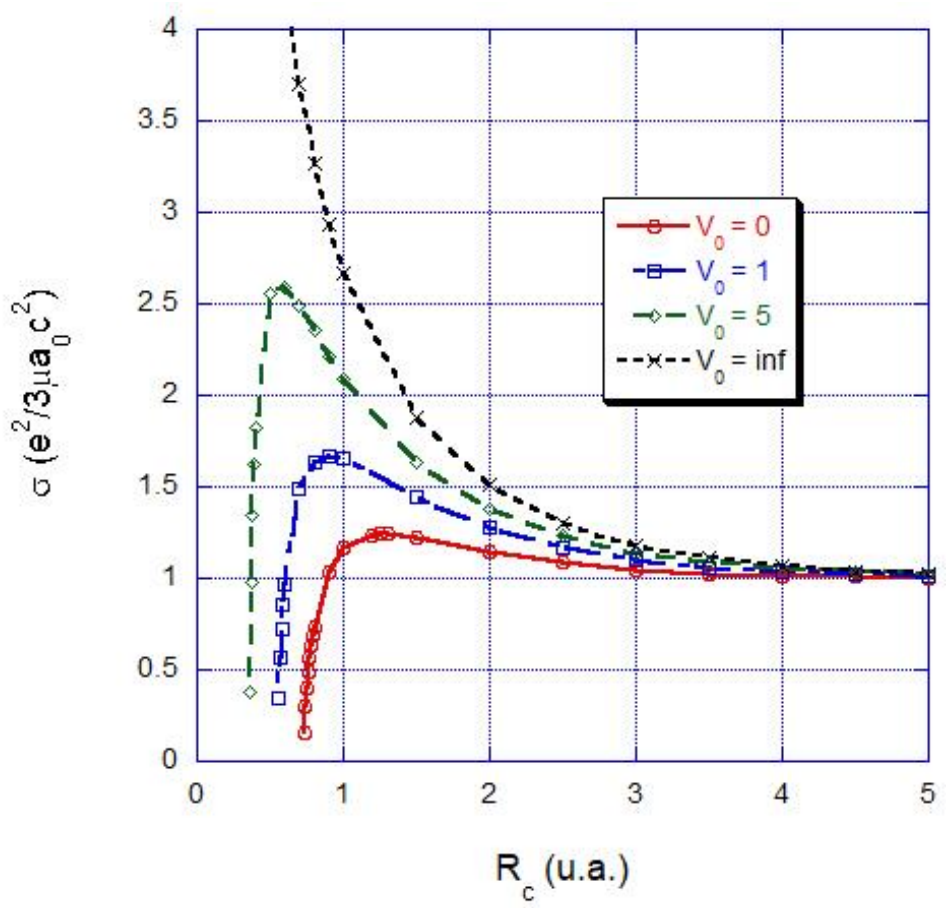

Figura 1.5. Constante de apantallamiento magnético (en unidades $e^{2} / 3 \mu a_{0} c^{2}$ ) como función de $R_{c}$ (bohrs) para $V_{0}=0,1,5, \infty$ hartrees.

En los cálculos de la polarizabilidad se usó la aproximación de Buckingham ya que se obtienen mejores resultados que con la aproximación de Kirkwood, normalmente utilizada en la literatura, incluso para valores grandes de $R_{c}$ [47]. Conforme el radio de confinamiento aumenta, ambas aproximaciones tienden a los mismos valores, así como para el caso límite $V_{0}=\infty$.

Para radios de confinamiento grandes la polarizabilidad, en la aproximación de Buckingham, tiende asintóticamente a su valor correspondiente en el caso libre de confinamiento, es decir, $\alpha_{d}=0.5927 \times 10^{-24} \mathrm{~cm}^{3}$. Cuando el radio de confinamiento decrece, $\alpha_{d}$ alcanza un valor mínimo, para subsecuentemente crecer rápidamente de manera ilimitada hasta que se alcanza el radio de confinamiento crítico. Por otra parte, para el caso de barrera impenetrable, la polarizabilidad tiende a cero cuando $R_{c}$ también tiende a cero. Opuesto a lo que pasa con el término de contacto de Fermi y con la constante de apantallamiento diamagnético, la 
polarizabilidad disminuye para paredes menos penetrables. En la Figura 1.6 se muestra el comportamiento de la polarizabilidad como función de $R_{c}$, para algunos valores de $V_{0}$.

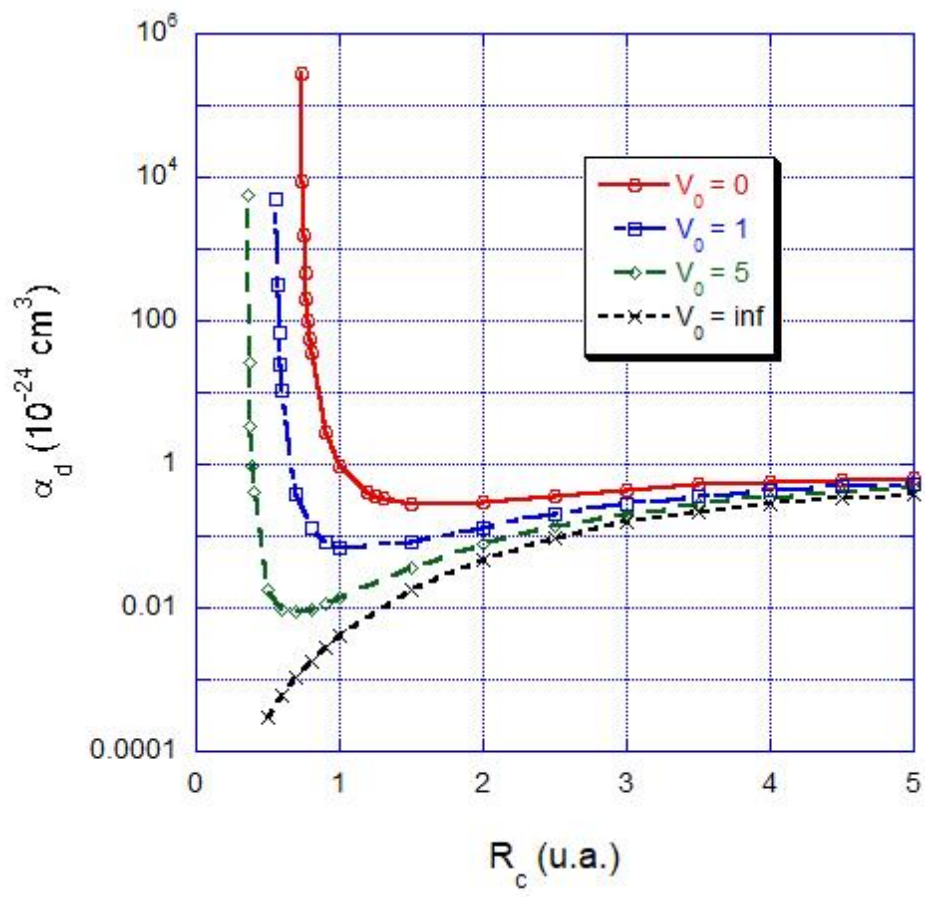

Figura 1.6. Polarizabilidad en la aproximación de Buckingham (en unidades $10^{-24} \mathrm{~cm}^{3}$ ) como función de $R_{c}$ (bohrs) para $V_{0}=0,1,5, \infty$ hartrees.

En trabajos previos [29, 31] la presión se calculó usando el teorema del virial

$$
P=\frac{1}{4 \pi R_{c}^{3}}(2 E-\langle V\rangle)
$$

Esta fórmula es correcta para el confinamiento dentro de paredes impenetrables. Sin embargo, como Fernández y Castro señalaron [108], el teorema del virial debe reformularse para sistemas con potenciales definidos a pedazos. En cualquier caso, la forma correcta de calcular la presión es mediante la ecuación (1.47). 
La derivada que aparece en la ecuación (1.47) es difícil de resolver de manera analítica, por lo que se calculó numéricamente. La diferenciación fue desarrollada usando la fórmula de diferenciación centrada con cinco términos [109]

$$
\left.\frac{d E}{d R_{c}}\right|_{R_{c}}=\frac{-E\left(R_{c}+2 h\right)+8 E\left(R_{c}+h\right)-8 E\left(R_{c}-h\right)+E\left(R_{c}-2 h\right)}{12 h}
$$

Con un tamaño de paso $h=0.01$.

En la Figura 1.7 se muestra el comportamiento de la presión como función de $R_{c}$ para algunos valores de $V_{0}$. Para un valor finito de $V_{0}$, la presión crece cuando $R_{c}$ disminuye, alcanza un valor máximo y luego decrece. Para una caja impenetrable, la presión crece sin límite cuando $R_{c} \rightarrow 0$.

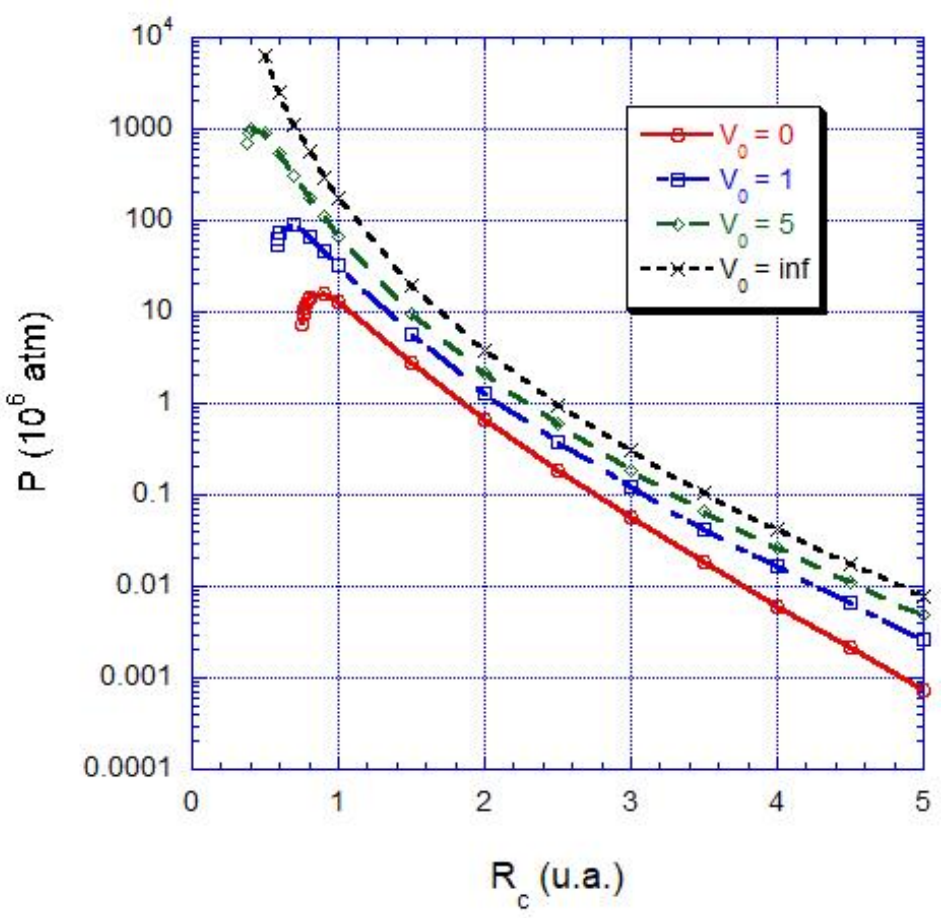

Figura 1.7. Presión $P$ (en unidades $10^{6}$ atm) como función de $R_{C}$ (bohrs) para $V_{0}=0,1,5, \infty$ hartrees. 
La ecuación (1.48) da la probabilidad de hallar al electrón en la región clásicamente prohibida, y es una medida del tunelamiento cuántico en esa región [40]. En la Figura 1.8 se muestra la probabilidad de tunelamiento del electrón como función de la altura de la barrera y el radio de confinamiento. Para un valor fijo de $V_{0}$, entre más confinado se encuentra el sistema, la probabilidad de tunelamiento crece acercándose a 1 , y decrece rápidamente cuando el radio de confinamiento aumenta.

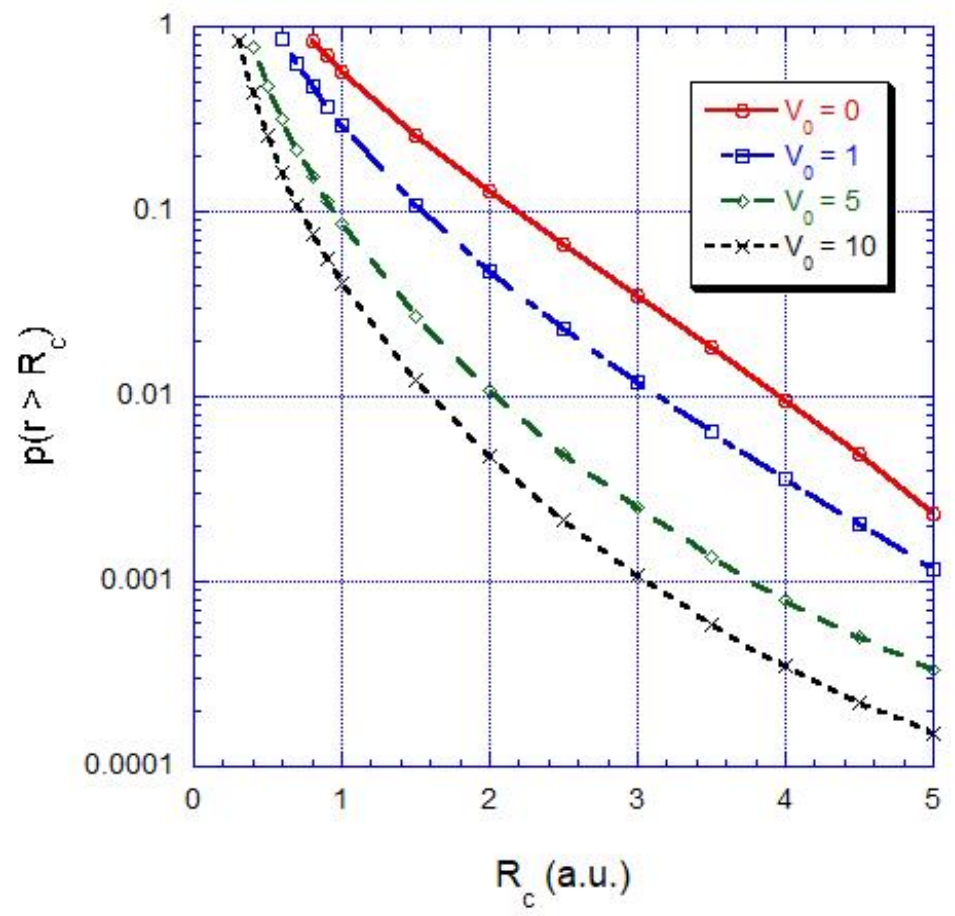

Figura 1.8. Probabilidad de tunelamiento como función de $R_{c}$ (bohrs) para $V_{0}=0,1,5,10$ hartrees. 


\subsection{Solución exacta}

\subsubsection{AHC dentro de paredes impenetrables}

El problema del átomo de hidrógeno confinado atrapado dentro de una cavidad esférica impenetrable tiene solución analíticamente exacta, y las soluciones numéricas más precisas fueron obtenidas por Aquino et al. [35]. Siguiendo el procedimiento aplicado en dicho trabajo, la ecuación de Schrödinger independiente del tiempo (en unidades atómicas) está dado por

$$
-\frac{1}{2} \nabla^{2} \psi+V(r) \psi=E \psi
$$

Donde

$$
V(r)=\left\{\begin{array}{rr}
-\frac{1}{r}, & r \leq R_{c} \\
\infty, & r>R_{c}
\end{array}\right.
$$

$R_{c}$ es el radio de confinamiento. Como es bien sabido para problemas con potenciales centrales, la función de onda puede separarse como el producto de una función de onda radial, multiplicada por los armónicos esféricos, dependientes de las coordenadas angulares

$$
\psi(r, \theta, \varphi)=R(r) Y_{l m}(\theta, \varphi)
$$

Sustituyendo la ecuación (1.53) en (1.51), se obtiene la ecuación de Schrödinger radial

$$
-\frac{1}{2 r^{2}} \frac{d}{d r}\left(r^{2} \frac{d}{d r} R(r)\right)+\left[V(r)+\frac{l(l+1)}{2 r^{2}}\right] R(r)=E R(r)
$$

Para la región de interés $0 \leq r \leq R_{c}$, la ecuación diferencial (1.54) se convierte en

$$
-\frac{1}{2 r^{2}} \frac{d}{d r}\left(r^{2} \frac{d}{d r} R(r)\right)+\left[\frac{l(l+1)}{2 r^{2}}-\frac{1}{r}\right] R(r)=E R(r)
$$

Con la condición de frontera de Dirichlet

$$
R\left(R_{c}\right)=0
$$

Haciendo las sustituciones

$$
\beta=\frac{1}{\sqrt{-2 E}}, \quad \rho=\frac{2 r}{\beta}
$$

Y proponiendo una solución de la forma 


$$
R=A e^{-\rho / 2} \rho^{l} F(\rho)
$$

La ecuación de Schrödinger radial (1.55) se convierte en

$$
\rho \frac{d^{2} F}{d \rho^{2}}+(2 l+2-\rho) \frac{d F}{d \rho}-(-\beta+l+1) F=0
$$

Ya que la función de onda debe ser finita en el origen, $F$ se trata de la función hipergeométrica confluente ${ }_{1} F_{1}(-\beta+l+1,2 l+2, \rho)$.

Ya que el átomo de hidrógeno se encuentra atrapado dentro de una esfera impenetrable de radio $R_{c}$, la condición de frontera de Dirichlet requiere que ${ }_{1} F_{1}=0$ en $r=R_{c}$. Por tanto las energías permitidas del sistema se hallan al encontrar las soluciones de la ecuación

$$
{ }_{1} F_{1}\left(-\beta+l+1,2 l+2, \rho_{c}\right)=0
$$

Donde $\rho_{c} \equiv 2 R_{c} / \beta$ y $\beta$ está relacionada con la energía $E$ a través de (1.57).

Para un valor dado del radio de confinamiento y del número cuántico $l$ la primera raíz corresponde al estado más bajo de energía $(n=1)$ y las raíces sucesivas corresponden a estados excitados. En este trabajo de tesis, los valores de la energía se calcularon con el programa Mathematica 9 para evaluar la función hipergeométrica confluente, y el procedimiento FindRoot para hallar las raíces de la ecuación (1.60), con una precisión de 25 cifras significativas. Estos resultados están en completa concordancia con aquellos de la referencia [35], al menos hasta esta cantidad de cifras, y se presentan en las Tablas 1.8 y 1.9 para el estado base y algunos estados excitados. Los eigenvalores de la energía $E_{n l}$ están etiquetados por el número cuántico principal $n$ y el momento angular $l$, como en el caso del átomo de hidrógeno libre de confinamiento.

Como puede observarse en las Tablas 1.8 y 1.9, la presencia de la cavidad impenetrable incrementa el valor de la energía. No sólo eso, sino que el confinamiento rompe parcialmente la degeneración de los estados del átomo de hidrógeno. Para una capa dada los estados con el valor más alto de momento angular $l$ son aquellos con la energía más baja. La degeneración de un nivel de energía $E_{n l}$ es de $2 l+1$, que corresponde a los posibles valores del número cuántico $m$. 
Tabla 1.8. Espectro de energías del AHC dentro de barreras impenetrables, como función del radio de confinamiento. Las energías están en hartrees y las distancias en bohrs.

\begin{tabular}{|c|c|c|}
\hline$R_{c}$ & $1 s$ & $2 s$ \\
\hline 0.5 & 14.747970030350280 & 72.672039190463577 \\
\hline 0.6 & 9.527707806146348 & 49.584719447589898 \\
\hline 0.7 & 6.469926127251262 & 35.779475675396125 \\
\hline 0.8 & 4.543380181009424 & 26.894321813254160 \\
\hline 0.9 & 3.262189536240119 & 20.854066669735000 \\
\hline 1.0 & 2.373990866103664 & 16.570256093469736 \\
\hline 1.5 & 0.437018065247256 & 6.644121826526449 \\
\hline 2.0 & -0.125000000000000 & 3.327509156496465 \\
\hline 2.5 & -0.334910185427921 & 1.865480181815459 \\
\hline 3.0 & -0.423967287733454 & 1.111684737436364 \\
\hline 4.0 & -0.483265302078022 & 0.420235631713674 \\
\hline 5.0 & -0.496417006591452 & 0.141254203802346 \\
\hline 7.0 & -0.499862577551406 & -0.051260393613739 \\
\hline 9.0 & -0.499995654219019 & -0.102834673891235 \\
\hline 10.0 & -0.499999263281525 & -0.112806210295841 \\
\hline$\infty$ & -0.500000000000000 & -0.125000000000000 \\
\hline & & \\
\hline
\end{tabular}


Tabla 1.9. Espectro de energías del AHC dentro de barreras impenetrables, como función del radio de confinamiento. Las energías están en hartrees y las distancias en bohrs.

\begin{tabular}{|c|c|c|c|}
\hline$R_{c}$ & $2 p$ & $3 p$ & $3 d$ \\
\hline 0.5 & 36.658875880189399 & 114.643552519280743 & 63.160184467371205 \\
\hline 0.6 & 24.936947036461358 & 78.955980782173932 & 43.405127502080990 \\
\hline 0.7 & 17.937632335905122 & 57.524686981433524 & 31.554058597378248 \\
\hline 0.8 & 13.439265252680276 & 43.671434967927300 & 23.901520992658518 \\
\hline 0.9 & 10.385635354292092 & 34.212367941938253 & 18.681850542981640 \\
\hline 1.0 & 8.223138316160864 & 27.473995302536328 & 14.967464086180532 \\
\hline 1.5 & 3.231051403227475 & 11.679573114684006 & 6.284819435316678 \\
\hline 2.0 & 1.576018785606341 & 6.269002791986478 & 3.327509156496465 \\
\hline 3.0 & 0.481250312526643 & 2.516209047333940 & 1.292803271991282 \\
\hline 4.0 & 0.143527083713958 & 1.261521214875995 & 0.621355776178396 \\
\hline 5.0 & 0.007593920467494 & 0.707718415822855 & 0.329117142966165 \\
\hline 7.0 & -0.087479017926819 & 0.257800616926432 & 0.096589640895385 \\
\hline 9.0 & -0.113727379564514 & 0.092571747874677 & 0.014006302118503 \\
\hline 10 & -0.118859544853860 & 0.049190760586633 & -0.007092783970206 \\
\hline$\infty$ & -0.125000000000000 & -0.055555555555556 & -0.055555555555556 \\
\hline
\end{tabular}

\subsubsection{Estructura fina del AHC}

Una vez resuelto el problema de eigenvalores y, obtenidas las funciones de onda del sistema, pueden calcularse los valores esperados y propiedades físicas que se deseen del sistema.

Cuando el radio de confinamiento disminuye, el electrón se mueve alrededor del núcleo en un espacio cada vez más pequeño, por lo que correspondientemente se incrementa la energía cinética, de acuerdo con el Principio de Incertidumbre de Heisenberg. Por tanto, se esperaría que la corrección relativista a las energías fueran mayores que para el átomo libre de confinamiento. En esta sección se analizarán las correcciones de estructura fina a las energías del átomo de hidrógeno confinado dentro de una barrera esférica de paredes impenetrables.

El hamiltoniano del AHC con efectos relativistas puede escribirse como 


$$
H=H^{(0)}+H^{\prime}
$$

Donde $H^{(0)}$ es el hamiltoniano no perturbado, asociado a la ecuación (1.51), y $H^{\prime}$ son las perturbaciones dadas por $[101,106]$

$$
H^{\prime}=H_{1}^{\prime}+H_{2}^{\prime}+H_{3}^{\prime}
$$

Donde

$$
\begin{gathered}
H_{1}^{\prime}=-\frac{\left(1 / 2 \nabla^{2}\right)^{2}}{2 c^{2}} \\
H_{2}^{\prime}=\frac{1}{2 c^{2} r} \frac{d V}{d r} \vec{L} \cdot \vec{S} \\
H_{3}^{\prime}=\frac{\pi}{2 c^{2}} \delta(\vec{r})
\end{gathered}
$$

El primer término, $H_{1}^{\prime}$, es debido a la corrección relativista a la energía cinética, el segundo término corresponde al acoplamiento espín-órbita y el tercero denota el término de Darwin.

Las funciones de onda de orden cero $\psi_{n l m_{l} m_{s}}^{(0)}$ son espín-orbitales construidas como el producto de un orbital espacial $\psi_{n l m_{l}}^{(0)}$ (dado por la ecuación (1.53)) y un espinor $\chi_{1 / 2, m_{s}}$, con $m_{s}= \pm 1 / 2$

$$
\psi_{n l m_{l} m_{s}}^{(0)}=\psi_{n l m_{l}}^{(0)} \chi_{1 / 2, m_{S}}
$$

Los orbitales $\psi_{n l m_{l}}^{(0)}$ cumplen con las condiciones de la ecuación de Schrödinger (1.51) con eigenvalores de la energía $E_{n l}^{(0)}$

$$
H^{(0)} \psi_{n l m_{l}}^{(0)}=E_{n l}^{(0)} \psi_{n l m_{l}}^{(0)}
$$

Los eigenvalores de la energía del sistema no perturbado $E_{n l}^{(0)}$ se obtienen como se explicó en la sección anterior.

Los espín-orbitales son eigenfunciones simultáneas de $H^{(0)}, L^{2}, L_{z}, S^{2}$ y $S_{z}$ con eigenvalores $E_{n l}^{(0)}, l(l+1), m_{l}, 3 / 4$ y $m_{s}$, respectivamente. Para cada nivel de energía $E_{n l}^{(0)}$ del AHC hay $2(2 l+1)$ espín-orbitales, donde $(2 l+1)$ corresponde a la degeneración del potencial de campo central y el factor 2 corresponde a los dos valores de espín de $m_{s}$. La degeneración del AHC es menor a la del átomo de hidrógeno libre $\left(2 n^{2}\right)$, la cual se recupera cuando $R_{c} \rightarrow$ $\infty$. 
El cálculo de la corrección a la energía usando teoría de perturbaciones a primer orden es directo:

La corrección relativista a la energía del AHC está dada por [101]

$$
E_{r}^{(1)}=-\frac{1}{2 c^{2}}\left[E_{n l}^{(0) 2}+2 E_{n l}^{(0)}\left\langle\frac{1}{r}\right\rangle_{n l}+\left\langle\frac{1}{r^{2}}\right\rangle_{n l}\right]
$$

Donde los valores esperados $\langle 1 / r\rangle_{n l} \mathrm{y}\left\langle 1 / r^{2}\right\rangle_{n l}$ se calculan usando las funciones de onda del AHC, dadas por las ecuaciones (1.53) y (1.58). En unidades atómicas $c=$ 137.03599911.

Ya que los niveles de energía $E_{n l}^{(0)}$ tienen una degeneración de $2(2 l+1)$, en principio debería utilizarse teoría de perturbaciones para estados degenerados para calcular la corrección a la energía a primer orden debida a la perturbación $H_{1}^{\prime}$. Sin embargo, puede observarse de la ecuación (1.63) que $H_{1}^{\prime}$ no actúa sobre la variable de espín. Además, conmuta con las componentes del momento angular orbital, por lo que los estados degenerados pertenecientes al nivel $E_{n l}^{(0)}$ no están conectados a primer orden por $H_{1}^{\prime}$. Por tanto, es válido el uso de teoría de perturbaciones para estados no degenerados.

En el caso de la perturbación $H_{2}^{\prime}$, de la ecuación (1.64) se observa que debido al término $\vec{L}$. $\vec{S}, H_{2}^{\prime}$ no conmuta con $L_{z}$ ni con $S_{z}$, por lo que se requeriría el uso de teoría de perturbaciones para estados degenerados para hallar la corrección a la energía debida a este término. Los cálculos pueden simplificarse utilizando una base de funciones adecuada, en la cual $H_{2}^{\prime}$ sea diagonal. De (1.64) puede verse que $H_{2}^{\prime}$ conmuta con $L^{2}, S^{2}$ y con el momento angular total

$$
\vec{J}=\vec{L}+\vec{S}
$$

Puesto en otra forma, las funciones $\psi_{\text {nljm }_{j}}^{(0)}$ construidas de la forma

$$
\psi_{n l j m_{j}}^{(0)}=\sum_{m_{l} m_{s}}\left\langle l s m_{l} m_{s} \mid j m_{j}\right\rangle \psi_{n l m_{l} m_{s}}^{(0)}, \quad s=\frac{1}{2}
$$

Donde $\left\langle l s m_{l} m_{s} \mid j m_{j}\right\rangle$ son los coeficientes de Clebsch-Gordan, son eigenfunciones simultáneas de $H^{(0)}, L^{2}, S^{2}, J^{2}$ y $J_{z}$, con eigenvalores $E_{n l}^{(0)}, l(l+1), 3 / 4, j(j+1)$ y $m_{j}$, respectivamente, con $j=|l-1 / 2|, \ldots, l+1 / 2$ y $m_{j}=-j, \ldots, 0, \ldots,+j$. En esta nueva base, 
el operador $\vec{L} \cdot \vec{S}$ (y por tanto $H_{2}^{\prime}$ ) es diagonal, y puede aplicarse teoría de perturbaciones para estados no degenerados para hallar la corrección a la energía.

Así, la corrección debida a la interacción espín-órbita está dada por

$$
E_{\text {so }}^{(1)}=\left\langle\psi_{\text {nljm }}^{(0)}\left|H_{2}^{\prime}\right| \psi_{n l j m_{j}}^{(0)}\right\rangle
$$

Reescribiendo el operador $\vec{L} \cdot \vec{S}$ como

$$
\vec{L} \cdot \vec{S}=\frac{1}{2}\left(J^{2}-L^{2}-S^{2}\right)
$$

La ecuación (1.71) queda como

$$
E_{\text {so }}^{(1)}=\frac{[j(j+1)-l(l+1)-3 / 4]}{4 c^{2}}\left\langle\frac{1}{r^{3}}\right\rangle_{n l}
$$

El valor esperado $\left\langle 1 / r^{3}\right\rangle$ se calcula con las funciones de onda radiales dadas por (1.58), ya que la parte radial de la solución de la ecuación de Schrödinger no se ve afectada por el cambio de base.

El término de Darwin sólo afecta los estados con simetría $S(l=0)$ ya que son los únicos distintos de cero en el origen

$$
E_{D}^{(1)}=\frac{\pi}{2 c^{2}}\left|\psi_{n 00}^{(0)}(0)\right|^{2}
$$

Este término está relacionado con el movimiento rápido (oscilaciones) del electrón alrededor del núcleo (zitterbewegung). Conforme el radio de confinamiento disminuye, la energía cinética del electrón aumenta, y la densidad electrónica alrededor del núcleo también crece, por lo que es de esperar también un incremento en el valor del término de Darwin.

De la ecuación (1.65) se observa que $H_{3}^{\prime}$ no actúa sobre la variable de espín y conmuta con $L^{2}$ y $L_{z}$, por lo que los estados degenerados pertenecientes al nivel $E_{n l}^{(0)}$ no están conectados a primer orden por $H_{3}^{\prime}$. Por tanto, es válido el uso de teoría de perturbaciones para estados no degenerados para hallar la corrección a la energía debida al término de Darwin, con las funciones de onda $\psi_{n l m_{l}}^{(0)}$.

Las energías del AHC considerando correcciones relativistas están dadas por 


$$
E_{n j}=E_{n l}^{(0)}+E_{r}^{(1)}+E_{s o}^{(1)}+E_{D}^{(1)}
$$

La corrección relativista a la energía cinética del electrón es pequeña comparada a la energía de su masa en reposo $m c^{2}=0.511 \mathrm{MeV}$. Por ejemplo, la razón para el estado $3 s$ del AHC en $R_{c}=0.5$ u.a., es aproximadamente $9.46 \times 10^{-3} \sim \alpha$. La ecuación (1.68) da por tanto la corrección a la energía cinética del electrón apropiadamente para radios de confinamiento $R_{c} \geq 0.5$ u.a.

Los estados con simetría S sólo tienen dos contribuciones relativistas: la corrección a la energía cinética y el término de Darwin. La primera se vuelve más negativa conforme $R_{c}$ tiende a cero, en tanto que el término de Darwin crece de manera positiva, como se observa en las Tablas 1.10 y 1.11 y en las Figuras 1.9 y 1.10. 
Tabla 1.10. Corrección relativista $E_{r}^{(1)}$ y término de Darwin $E_{D}^{(1)}$ del estado base (1s) del AHC como función del radio de confinamiento $R_{c}$. Las energías están en hartrees y los radios en bohrs.

\begin{tabular}{|c|c|c|}
\hline$R_{C}$ & $E_{r}^{(1)}$ & $E_{D}^{(1)}$ \\
\hline 0.5 & -0.010890946296549 & 0.001463254489289 \\
\hline 0.6 & -0.005375519223353 & 0.000905292380175 \\
\hline 0.7 & -0.002984746976474 & 0.000609602114667 \\
\hline 0.8 & -0.001809109908346 & 0.000436766727014 \\
\hline 0.9 & -0.001173972429158 & 0.000328130958098 \\
\hline 1.0 & -0.000804830730881 & 0.000255918235918 \\
\hline 1.5 & -0.000213117125238 & 0.000106493731468 \\
\hline 2.0 & -0.000099508667876 & 0.000063210236046 \\
\hline 2.5 & -0.000063416196423 & 0.000045498764399 \\
\hline 3.0 & -0.000048435737783 & 0.000036871046555 \\
\hline 4.0 & -0.000037491951438 & 0.000029718862707 \\
\hline 5.0 & -0.000034420573658 & 0.000027498833529 \\
\hline 7.0 & -0.000033345380894 & 0.000026676502553 \\
\hline 9.0 & -0.000033284694084 & 0.000026627821471 \\
\hline 10.0 & -0.000033282585428 & 0.000026626085063 \\
\hline$\infty$ & -0.000033282096549 & 0.000026625677239 \\
\hline & & \\
\hline
\end{tabular}


Tabla 1.11. Corrección relativista $E_{r}^{(1)}$ y término de Darwin $E_{D}^{(1)}$ del estado $2 s$ del AHC como función del radio de confinamiento $R_{c}$. Las energías están en hartrees y los radios en bohrs.

\begin{tabular}{|c|c|c|}
\hline$R_{C}$ & $E_{r}^{(1)}$ & $E_{D}^{(1)}$ \\
\hline 0.5 & -0.167298589680307 & 0.005113083160083 \\
\hline 0.6 & -0.080973172259490 & 0.003075019276895 \\
\hline 0.7 & -0.043898664542648 & 0.002011907924572 \\
\hline 0.8 & -0.025865105706487 & 0.001399969717160 \\
\hline 0.9 & -0.016243417934079 & 0.001021002691761 \\
\hline 1.0 & -0.010729298009307 & 0.000772668436337 \\
\hline 1.5 & -0.002219640464883 & 0.000274643815903 \\
\hline 2.0 & -0.000751306215374 & 0.000137651092836 \\
\hline 2.5 & -0.000335761443790 & 0.000082745954038 \\
\hline 3.0 & -0.000179528158724 & 0.000055454545198 \\
\hline 4.0 & -0.000071790211447 & 0.000029962665678 \\
\hline 5.0 & -0.000037422903885 & 0.000018509573467 \\
\hline 7.0 & -0.000015420788505 & 0.000008848678516 \\
\hline 9.0 & -0.000009048909386 & 0.000005448117813 \\
\hline 10.0 & -0.000007636124840 & 0.000004643707311 \\
\hline$\infty$ & -0.000005408340689 & 0.000003328209655 \\
\hline & & \\
\hline
\end{tabular}



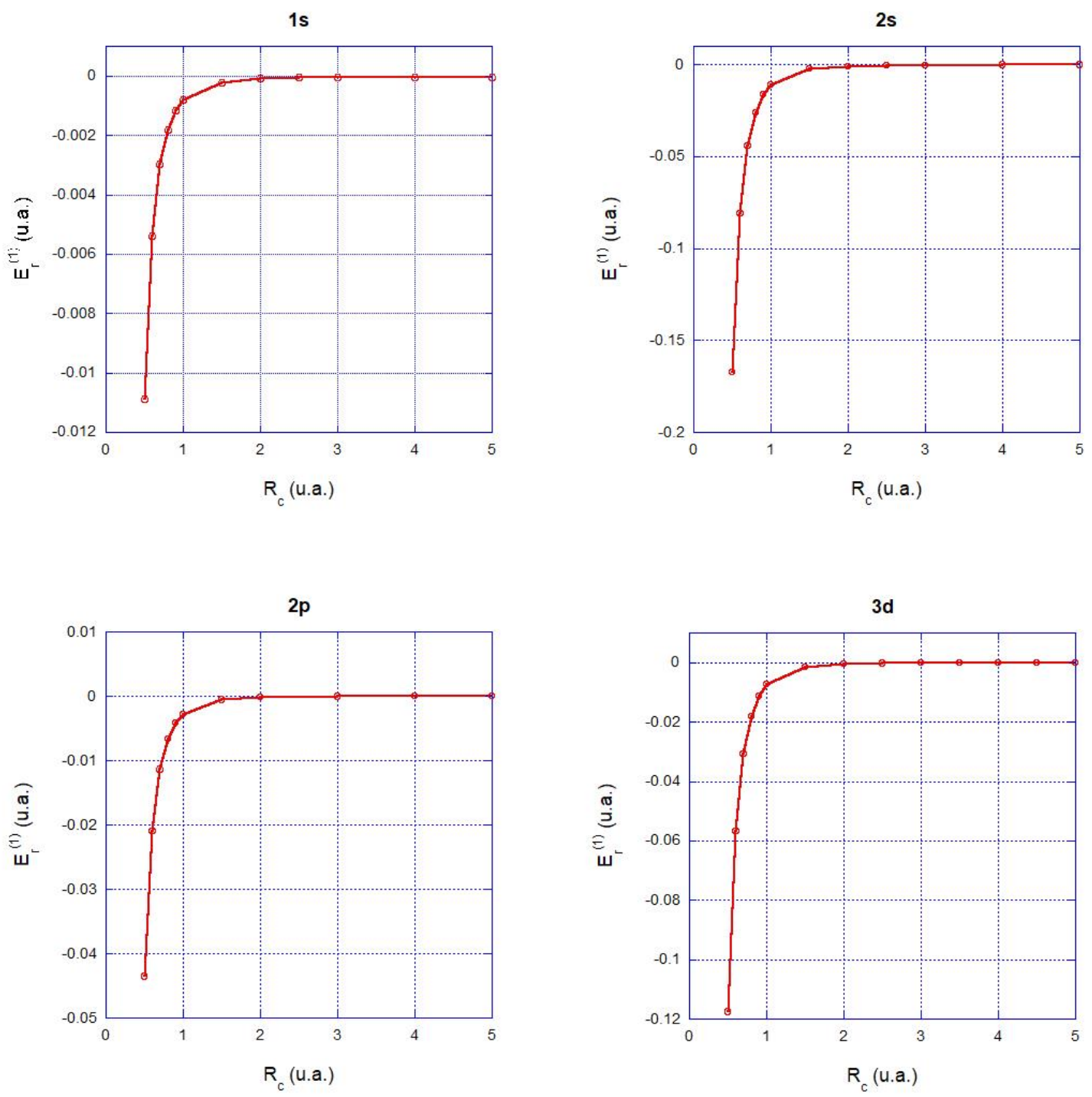

Figura 1.9. Correcciones relativistas a la energía cinética $E_{r}^{(1)}$ para los estados $1 s, 2 s, 2 p$ y $3 d$ del AHC como función de $R_{c}$. 

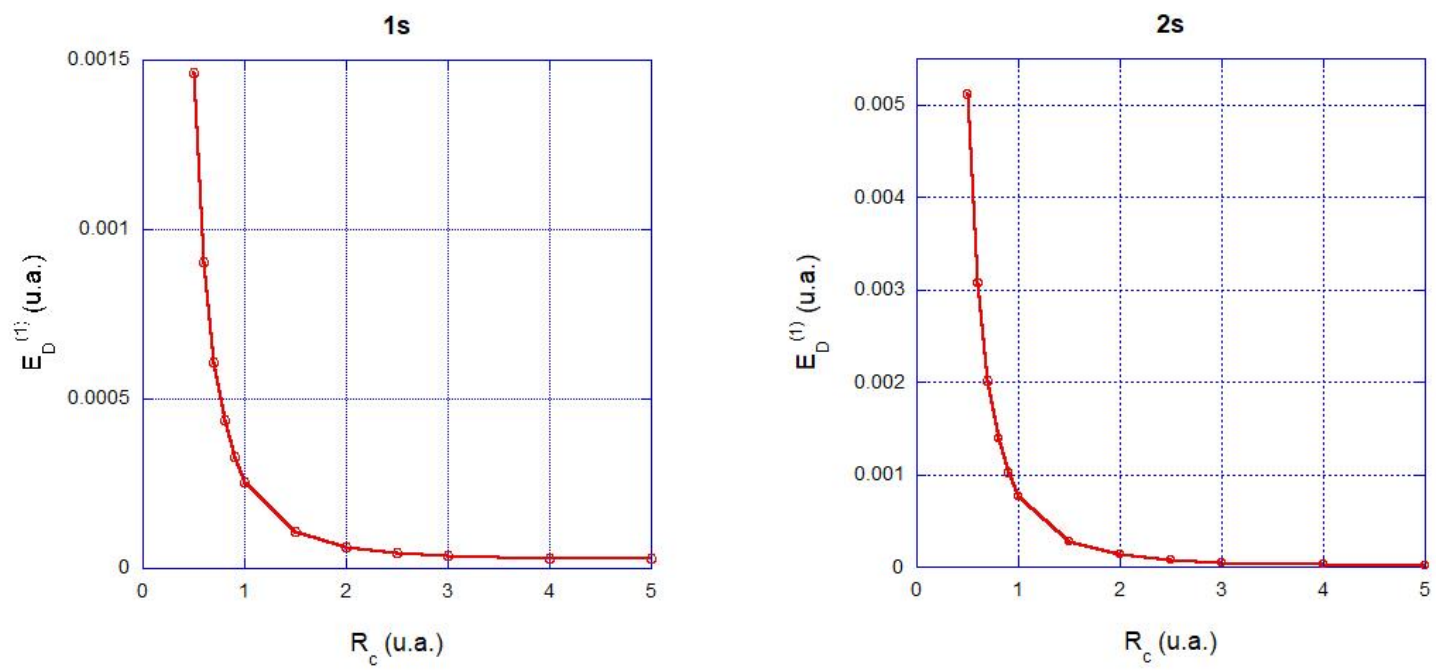

Figura 1.10. Término de Darwin para los estados $1 s$ y $2 s$ del AHC como función de $R_{c}$. Las energías están en hartrees y los radios en bohrs.

Los estados con $l>0$ sólo tienen correcciones relativistas a la energía cinética y por el acoplamiento espín-órbita. Para el átomo de hidrógeno libre los niveles $2 s_{1 / 2}$ y $2 p_{1 / 2}$ poseen la misma energía, es decir, están degenerados. En la teoría de Dirac, los estados con mismos números cuánticos $(n, j)$ tal que $j=l \pm 1 / 2$, también tienen la misma energía. Esta degeneración es removida cuando se consideran efectos de electrodinámica cuántica.

Sin embargo, para el átomo de hidrógeno confinado, la degeneración en los estados dentro de una misma capa con diferente valor de $l$ es removida parcialmente debido a la repulsión de la pared impenetrable. Por ejemplo, los estados $2 s$ y $2 p$ del AHC no están degenerados para $R_{c}<10$ u.a. La diferencia en las energías $\Delta E=E_{20}-E_{21}$ como función de $R_{c}$, es algunos órdenes de magnitud mayor que las correcciones relativistas (ver las Tablas $1.12 \mathrm{y}$ 1.14). Por esta razón el estado $2 s_{1 / 2}$ tendrá una energía diferente comparada a la de los estados $2 p_{1 / 2}$ y $2 p_{3 / 2}$. Además, las correcciones perturbativas de los estados $2 p_{1 / 2}$ y $2 p_{3 / 2}$ tienen valores diferentes (ver Tabla 1.12 y Figura 1.11). Por tanto, estos estados tendrán energías diferentes para cajas con $R_{c}<10$ u.a. 
Tabla 1.12. Corrección relativista $E_{r}^{(1)}$ y corrección de acoplamiento espín-órbita $E_{s o}^{(1)}$ del estado $2 p$ del AHC como función del radio de confinamiento $R_{c}$. Las energías están en hartrees y los radios en bohrs.

\begin{tabular}{|c|c|c|c|}
\hline$R_{c}$ & $E_{r}^{(1)}$ & $E_{s o}^{(1)}(j=1 / 2)$ & $E_{s o}^{(1)}(j=3 / 2)$ \\
\hline 0.5 & -0.043517169403930 & -0.002265272040865 & 0.001132636020433 \\
\hline 0.6 & -0.021008199227508 & -0.001325069125125 & 0.000662534562563 \\
\hline 0.7 & -0.011353910155357 & -0.000843544280752 & 0.000421772140376 \\
\hline 0.8 & -0.006665222920648 & -0.000571333919743 & 0.000285666959872 \\
\hline 0.9 & -0.004168093747417 & -0.000405731831501 & 0.000202865915751 \\
\hline 1.0 & -0.002739926750033 & -0.000299104091074 & 0.000149552045537 \\
\hline 1.5 & -0.000548408560341 & -0.000093881178309 & 0.000046940589154 \\
\hline 2.0 & -0.000176991724021 & -0.000042080552352 & 0.000021040276176 \\
\hline 3.0 & -0.000037218817367 & -0.000014206835621 & 0.000007103417810 \\
\hline 4.0 & -0.000012985175640 & -0.000006918569911 & 0.000003459284956 \\
\hline 5.0 & -0.000006096735194 & -0.000004144104146 & 0.000002072052073 \\
\hline 7.0 & -0.000002339923559 & -0.000002149539183 & 0.000001074769591 \\
\hline 9.0 & -0.000001424244382 & -0.000001504323362 & 0.000000752161681 \\
\hline 10 & -0.000001237001261 & -0.000001352018201 & 0.000000676009100 \\
\hline$\infty$ & -0.000000970727816 & -0.000001109403218 & 0.000000554701609 \\
\hline & & & \\
\hline
\end{tabular}


Tabla 1.13. Corrección relativista $E_{r}^{(1)}$ y corrección de acoplamiento espín-órbita $E_{s o}^{(1)}$ del estado $3 p$ del AHC como función del radio de confinamiento $R_{c}$. Las energías están en hartrees y los radios en bohrs.

\begin{tabular}{|c|c|c|c|}
\hline$R_{c}$ & $E_{r}^{(1)}$ & $E_{\text {so }}^{(1)}(j=1 / 2)$ & $E_{\text {so }}^{(1)}(j=3 / 2)$ \\
\hline 0.5 & -0.379614356559985 & -0.006622832029955 & 0.003311416014978 \\
\hline 0.6 & -0.183132820296056 & -0.003864656625314 & 0.001932328312657 \\
\hline 0.7 & -0.098890836199988 & -0.002454111285895 & 0.001227055642947 \\
\hline 0.8 & -0.057995490624988 & -0.001657886403586 & 0.000828943201793 \\
\hline 0.9 & -0.036225926404992 & -0.001174210759387 & 0.000587105379693 \\
\hline 1.0 & -0.023782349418593 & -0.000863246389890 & 0.000431623194945 \\
\hline 1.5 & -0.004717147734461 & -0.000266898332410 & 0.000133449166205 \\
\hline 2.0 & -0.001501509065069 & -0.000117569586662 & 0.000058784793331 \\
\hline 3.0 & -0.000302010496151 & -0.000038043281151 & 0.000019021640575 \\
\hline 4.0 & -0.000098167187962 & -0.000017556868593 & 0.000008778434296 \\
\hline 5.0 & -0.000041698731587 & -0.000009841784678 & 0.000004920892339 \\
\hline 7.0 & -0.000012012094837 & -0.000004292526224 & 0.000002146263112 \\
\hline 9.0 & -0.000005030752490 & -0.000002392911366 & 0.000001196455683 \\
\hline 10 & -0.000003564117674 & -0.000001886378070 & 0.000000943189035 \\
\hline$\infty$ & -0.000000410890081 & -0.000000328712065 & 0.000000164356032 \\
\hline
\end{tabular}


Tabla 1.14. Corrección relativista $E_{r}^{(1)}$ y corrección de acoplamiento espín-órbita $E_{s o}^{(1)}$ del estado $3 d$ del AHC como función del radio de confinamiento $R_{c}$. Las energías están en hartrees y los radios en bohrs.

\begin{tabular}{|c|c|c|c|c|}
\hline$R_{c}$ & $E_{r}^{(1)}$ & $E_{\text {so }}^{(1)}(j=1 / 2)$ & $E_{\text {so }}^{(1)}(j=3 / 2)$ & $E_{\text {so }}^{(1)}(j=5 / 2)$ \\
\hline 0.5 & -0.117564097569745 & -0.003605091194179 & -0.001802545597089 & 0.001201697064726 \\
\hline 0.6 & -0.056706232917823 & -0.002094309431948 & -0.001047154715974 & 0.000698103143983 \\
\hline 0.7 & -0.030615417553003 & -0.001323973105667 & -0.000661986552833 & 0.000441324368556 \\
\hline 0.8 & -0.017950835348891 & -0.000890414939098 & -0.000445207469549 & 0.000296804979699 \\
\hline 0.9 & -0.011209927193594 & -0.000627819063718 & -0.000313909531859 & 0.000209273021239 \\
\hline 1.0 & -0.007357279283167 & -0.000459485964342 & -0.000229742982171 & 0.000153161988114 \\
\hline 1.5 & -0.001456551256460 & -0.000138902920606 & -0.000069451460303 & 0.000046300973535 \\
\hline 2.0 & -0.000462366927413 & -0.000059825371435 & -0.000029912685717 & 0.000019941790478 \\
\hline 2.5 & -0.000190211994013 & -0.000031292022908 & -0.000015646011454 & 0.000010430674303 \\
\hline 3.0 & -0.000092238821654 & -0.000018512590938 & -0.000009256295469 & 0.000006170863646 \\
\hline 4.0 & -0.000029623333499 & -0.000008179898627 & -0.000004089949313 & 0.000002726632876 \\
\hline 5.0 & -0.000012386627300 & -0.000004399960086 & -0.000002199980043 & 0.000001466653362 \\
\hline 7.0 & -0.000003428293660 & -0.000001787758862 & -0.000000893879431 & 0.000000595919621 \\
\hline 9.0 & -0.000001376736989 & -0.000000951897935 & -0.000000475948968 & 0.000000317299312 \\
\hline 10 & -0.000000959043756 & -0.000000742709011 & -0.000000371354506 & 0.000000247569670 \\
\hline$\infty$ & -0.000000147920429 & -0.000000197227239 & -0.000000098613619 & 0.000000065742413 \\
\hline
\end{tabular}



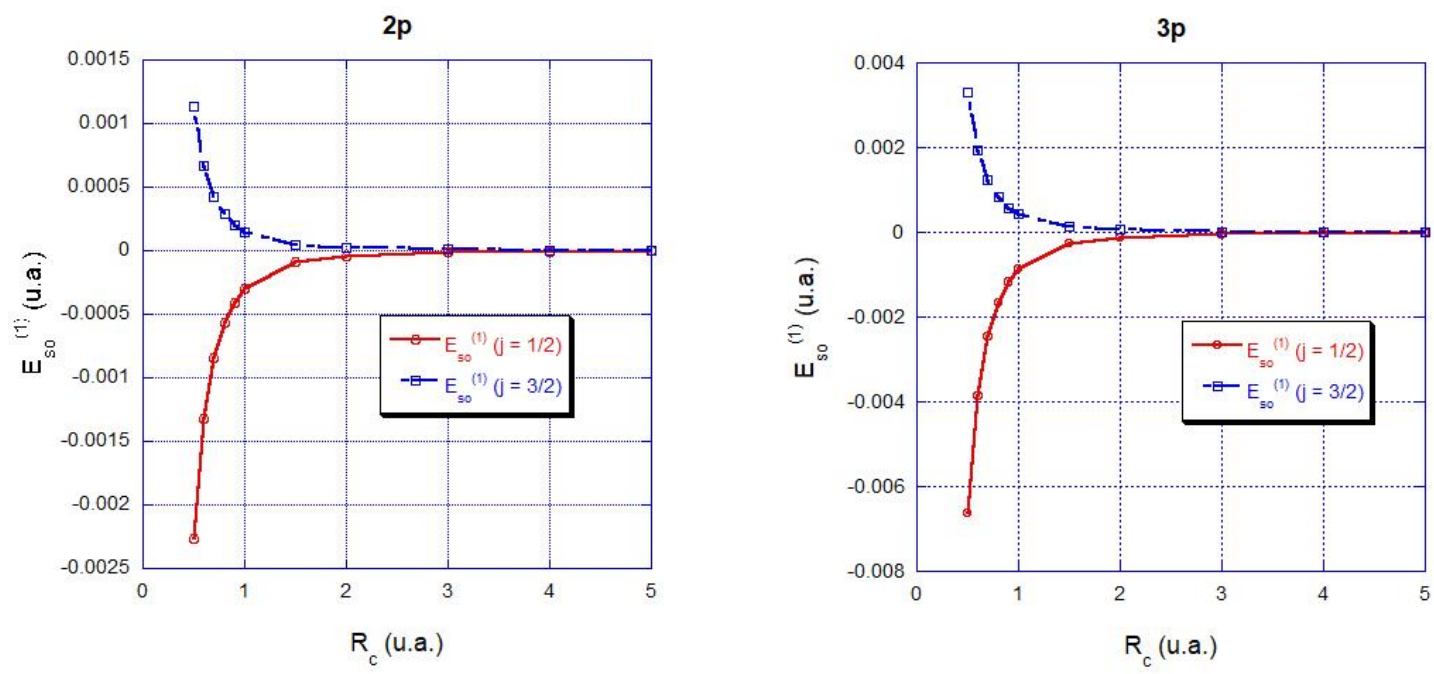

Figura 1.11. Acoplamiento espín-órbita $E_{s o}^{(1)}$ para los estados $2 p$ y $3 p$ del AHC como función de $R_{c}$. Las energías están en hartrees y los radios en bohrs.

En cuanto a la capa $n=3$, como se muestra en la Tabla 1.13, la corrección de acoplamiento espín-órbita es menor para el estado $3 p_{1 / 2}$, en comparación al estado $3 p_{3 / 2}$ (ver Figura 1.11). Mientras que para el estado $3 d$, el orden de la corrección de acoplamiento espín-órbita es $E_{s o}^{(1)}\left(3 d_{1 / 2}\right)<E_{s o}^{(1)}\left(3 d_{3 / 2}\right)<E_{s o}^{(1)}\left(3 d_{5 / 2}\right)$, para todos los valores de $R_{c}$ aquí considerados (Tabla 1.14 y Figura 1.12). Por tanto para estados con los mismos valores de $n$ y $l$, aquél con el valor de $j$ más bajo tiene la corrección de acoplamiento espín-órbita menor. 


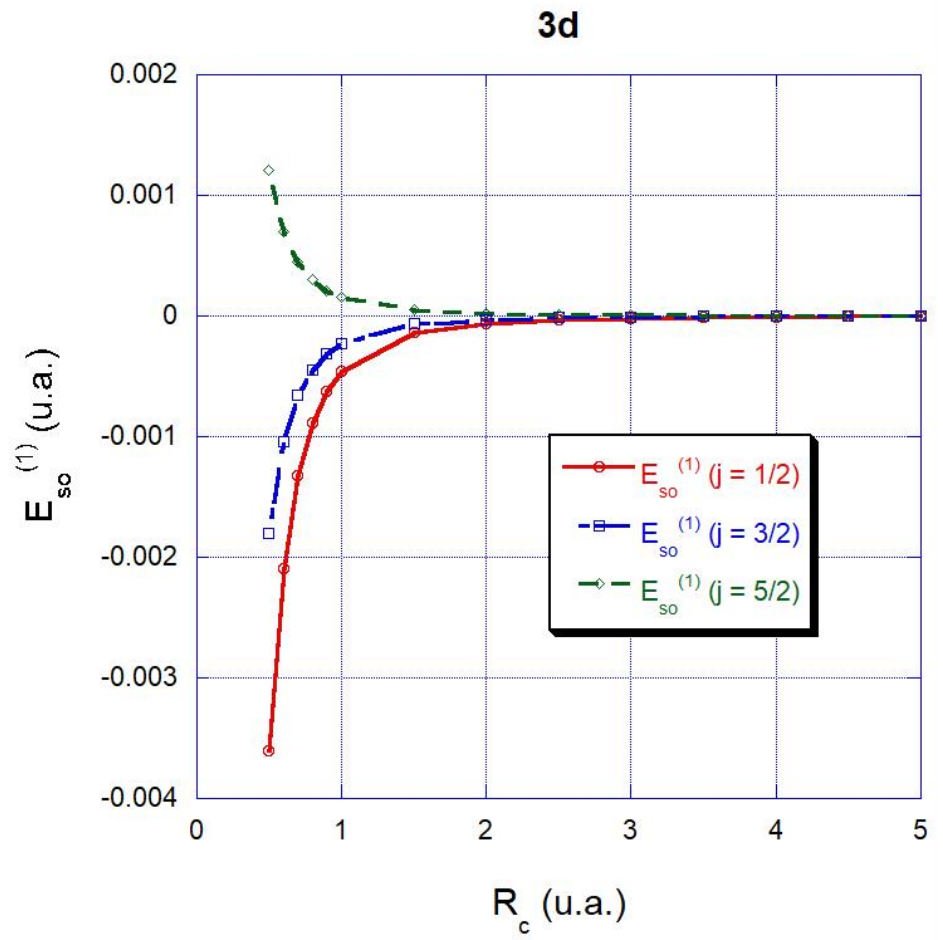

Figura 1.12. Acoplamiento espín-órbita para el estado $3 d$ del AHC como función del radio de confinamiento.

\subsubsection{AHC dentro de paredes penetrables}

De igual manera que en el caso del átomo de hidrógeno confinado dentro de una esfera de paredes impenetrables, cuando el átomo se confina dentro de una esfera de paredes suaves (penetrables) el problema puede resolverse de forma analítica, en términos de funciones hipergeométricas confluentes. Esto se logra como se muestra a continuación.

En unidades atómicas $(m=e=\hbar=1)$, la ecuación de Schrödinger de un átomo de hidrógeno, cuyo núcleo se encuentra fijo en el centro de una esfera de radio $R_{c}$ y confinado por un potencial constante $V_{0}$ es

$$
\left(-\frac{1}{2} \nabla^{2}+V(r)\right) \psi(r, \theta, \varphi)=E \psi(r, \theta, \varphi)
$$

Donde el potencial $V(r)$ es 


$$
V(r)=\left\{\begin{array}{cc}
-\frac{1}{r}, & r \leq R_{c} \\
V_{0}, & r>R_{C}
\end{array}\right.
$$

La ecuación (1.76) puede resolverse usando separación de variables

$$
\psi(r, \theta, \varphi)=R(r) Y_{l m}(\theta, \varphi)
$$

La función de onda radial $R(r)$ es una función a pedazos compuesta por la función $R_{i}(r)$, dentro de la esfera, y $R_{e}(r)$, la función de onda fuera de la esfera.

La ecuación de Schrödinger radial para la región interior $0 \leq r \leq R_{c}$ es

$$
\left[-\frac{1}{2}\left(\frac{1}{r^{2}} \frac{d}{d r} r^{2} \frac{d}{d r}\right)+\frac{l(l+1)}{2 r^{2}}-\frac{1}{r}\right] R_{i}(r)=E R_{i}(r)
$$

Cuya solución está dada por (ver Sección 1.2.1 y referencias [35, 47])

$$
R_{i}(r)=A e^{-\rho / 2} \rho_{1}^{l} F_{1}(-\beta+l+1,2 l+2, \rho)
$$

Donde ${ }_{1} F_{1}$ es la función hipergeométrica confluente y

$$
\beta=\frac{1}{\sqrt{-2 E}}, \quad \rho=\frac{2 r}{\beta}
$$

La región externa, $r>R_{c}$, está descrita por la ecuación de Schrödinger radial

$$
\left[-\frac{1}{2}\left(\frac{1}{r^{2}} \frac{d}{d r} r^{2} \frac{d}{d r}\right)+\frac{l(l+1)}{2 r^{2}}+V_{0}\right] R_{e}(r)=E R_{e}(r)
$$

La cual puede escribirse como

$$
\left(\frac{d^{2}}{d y^{2}}+\frac{2}{y} \frac{d}{d y}-\frac{l(l+1)}{y^{2}}-1\right) R_{e}(y)=0
$$

Donde

$$
y=k r, \quad k^{2}=2\left(V_{0}-E\right)
$$

Y cuya solución está dada por [31]

$$
R_{e}(y)=B y^{-l-1} e^{-y} F_{1}(-l,-2 l, 2 y)
$$

Los eigenvalores de la energía de este sistema son obtenidos con el requerimiento de que la función de onda debe ser continua, con primera derivada continua en la frontera $r=R_{c}$. Esta condición es más sencilla de aplicar igualando las derivadas logarítmicas en $R_{c}$, resultando en la ecuación 


$$
\left.\frac{d}{d r}\left[\ln R_{i}\right]\right|_{R_{c}}-\left.\frac{d}{d r}\left[\ln R_{e}\right]\right|_{R_{c}}=0
$$

Las constantes de normalización $A$ y $B$ se encuentran usando la condición de continuidad

$$
R_{i}\left(\rho_{c} \equiv \frac{2 R_{c}}{\beta}\right)=R_{e}\left(y_{c} \equiv k R_{c}\right)
$$

Y la condición de normalización

$$
\int_{0}^{R_{c}} R_{i}^{2} r^{2} d r+\int_{R_{c}}^{\infty} R_{e}^{2} r^{2} d r=1
$$

Para un valor fijo del momento angular $l$, el radio de confinamiento $R_{c}$ y la altura de la barrera $V_{0}$, las raíces de la ecuación (1.86) son los eigenvalores de la energía del AHC. El primer cero corresponde a la energía del estado más bajo, y el resto de raíces son las energías de estados excitados. Con estos valores, se construye la función de onda en cada región. Para hallar las raíces de la ecuación (1.86) se usó el comando FindRoot del programa Mathematica 9 con variables con 50 dígitos de precisión. Los eigenvalores obtenidos por este procedimiento se muestran en las Tablas 1.15 a 1.17 como funciones del radio de la caja $R_{c}$ y la altura del potencial $V_{0}$. Estos resultados concuerdan con los obtenidos por otros autores $[17,31,47]$. Los resultados se muestran con 15 dígitos después del punto decimal. En la Tabla 1.15 también se muestra el radio crítico para el cual la energía del estado base es igual a la altura de la barrera, es decir, $E_{10}=V_{0}$. Para radios menores a éste, el electrón escapa del confinamiento y se convierte en una partícula libre. 
Tabla 1.15. Energía del estado base del AHC como función del radio de confinamiento $R_{c}$ y la altura de la barrera $V_{0}$. Las energías están en hartrees y las distancias en bohrs.

\begin{tabular}{|c|c|c|c|}
\hline$R_{c}$ & $1 s$ & $R_{c}$ & $1 s$ \\
\hline \multicolumn{2}{|c|}{$V_{0}=0$} & \multicolumn{2}{|c|}{$V_{0}=1$} \\
\hline 0.72289826153284 & 0 & 0.55037431535838 & 1 \\
\hline 0.8 & -0.017424391031037 & 0.8 & 0.612656360215074 \\
\hline 0.9 & -0.067406311452319 & 0.9 & 0.433928950530631 \\
\hline 1.0 & -0.125000000000000 & 1.0 & 0.280173920470822 \\
\hline 2.0 & -0.431218889241793 & 2.0 & -0.357062118292440 \\
\hline 2.5 & -0.470393522970229 & 2.5 & -0.434538985097672 \\
\hline 3.0 & -0.487223082818398 & 3.0 & -0.469568280998335 \\
\hline 3.5 & -0.494519692585279 & 3.5 & -0.485847181581401 \\
\hline 4.0 & -0.497674689400819 & 4.0 & -0.493472717471213 \\
\hline 4.5 & -0.499025598864902 & 4.5 & -0.497028516691869 \\
\hline 5.0 & -0.499596671366575 & 5.0 & -0.498667227455794 \\
\hline \multicolumn{2}{|c|}{$V_{0}=5$} & \multicolumn{2}{|c|}{$V_{0}=10$} \\
\hline 0.356704984274150 & 5 & 0.277574665930449 & 10 \\
\hline 0.4 & 4.827691517791768 & 0.4 & 7.729216960720511 \\
\hline 0.5 & 3.907609648085746 & 0.5 & 5.639829938566619 \\
\hline 0.6 & 2.982382648801240 & 0.6 & 4.097374126091258 \\
\hline 0.7 & 2.238447212017127 & 0.7 & 2.995752750577570 \\
\hline 0.8 & 1.666642892127310 & 0.8 & 2.202173313199984 \\
\hline 0.9 & 1.229573168163173 & 0.9 & 1.620350180030345 \\
\hline 1.0 & 0.893377387585463 & 1.0 & 1.185777102316168 \\
\hline 1.5 & 0.028688218476904 & 1.5 & 0.116618456909048 \\
\hline 2.0 & -0.273954162644265 & 2.0 & -0.240385375166330 \\
\hline 2.5 & -0.397386813501905 & 2.5 & -0.382937517117798 \\
\hline 3.0 & -0.452163079706601 & 3.0 & -0.445545908145495 \\
\hline 3.5 & -0.477542879189045 & 3.5 & -0.474425028203553 \\
\hline 4.0 & -0.489507801753420 & 4.0 & -0.488027526391939 \\
\hline 4.5 & -0.495153894267392 & 4.5 & -0.494454829661503 \\
\hline 5.0 & -0.497794417047201 & 5.0 & -0.497468459482318 \\
\hline
\end{tabular}


Tabla 1.16. Energía de algunos estados excitados del AHC como función del radio de confinamiento y la altura de la barrera. Las energías están en hartrees y las distancias en bohrs.

\begin{tabular}{|c|c|c|}
\hline$R_{C}$ & $2 s$ & $3 s$ \\
\hline & \multicolumn{2}{|c|}{$V_{0}=0$} \\
\hline 4.0 & -0.003721181517809 & \\
\hline 4.5 & -0.026821985024274 & \\
\hline 5.0 & -0.050466554191376 & \\
\hline 7.0 & -0.103067269500966 & \\
\hline 9.0 & -0.118876046657525 & \\
\hline \multirow[t]{2}{*}{10.0} & -0.121834827144001 & -0.004717416004277 \\
\hline & \multicolumn{2}{|c|}{$V_{0}=1$} \\
\hline 2.5 & 0.839527477867719 & \\
\hline 3.0 & 0.551921333772435 & \\
\hline 3.5 & 0.346631646525790 & \\
\hline 4.0 & 0.207031072469162 & \\
\hline 4.5 & 0.111527417715882 & 0.877941382224329 \\
\hline 5.0 & 0.045275020454115 & 0.703021591511976 \\
\hline 7.0 & -0.075871410202790 & 0.281770508851761 \\
\hline 9.0 & -0.110151424361619 & 0.107762108355053 \\
\hline \multirow[t]{2}{*}{10.0} & -0.116876495276936 & 0.060582479800732 \\
\hline & \multicolumn{2}{|c|}{$V_{0}=5$} \\
\hline 1.5 & 3.924908796636212 & \\
\hline 2.0 & 2.238120253638942 & \\
\hline 2.5 & 1.327970075169425 & 4.093616199785006 \\
\hline 3.0 & 0.813037500004446 & 2.879806387749310 \\
\hline 3.5 & 0.502902339789481 & 2.075569844531725 \\
\hline 4.0 & 0.306645277650973 & 1.534386351680299 \\
\hline 4.5 & 0.177709256169596 & 1.158153768884154 \\
\hline 5.0 & 0.090550968134425 & 0.888561588930425 \\
\hline 7.0 & -0.064063824530391 & 0.338443219377837 \\
\hline 9.0 & -0.106605912855536 & 0.131078976550960 \\
\hline \multirow[t]{2}{*}{10.0} & -0.114899798518405 & 0.076399115194474 \\
\hline & \multicolumn{2}{|c|}{$V_{0}=10$} \\
\hline 1.0 & 9.356033302760599 & \\
\hline 1.5 & 4.673654824749035 & \\
\hline 2.0 & 2.522714427940619 & 7.101418325006743 \\
\hline 2.5 & 1.467614340606850 & 4.594852658598024 \\
\hline 3.0 & 0.890881390314687 & 3.130819798341389 \\
\hline 3.5 & 0.549876152298087 & 2.223516879854503 \\
\hline 4.0 & 0.336555510833510 & 1.629431697044926 \\
\hline 4.5 & 0.197496743587726 & 1.222783494234172 \\
\hline 5.0 & 0.104016398299173 & 0.934357122024305 \\
\hline 7.0 & -0.060624006953468 & 0.353390987426017 \\
\hline 9.0 & -0.105586533048646 & 0.137269666355284 \\
\hline 10.0 & -0.114333229990090 & 0.080595153934551 \\
\hline
\end{tabular}


Tabla 1.17. Energía de estados excitados del átomo de hidrógeno confinado como función del radio de confinamiento y la altura de la barrera. Las energías están en hartrees y las longitudes en bohrs.

\begin{tabular}{|c|c|c|c|}
\hline$R_{C}$ & $2 p$ & $3 p$ & $3 d$ \\
\hline & \multicolumn{3}{|c|}{$V_{0}=0$} \\
\hline 4.0 & -0.043681740802597 & & \\
\hline 5.0 & -0.082623935222285 & & \\
\hline 7.0 & -0.113391976864806 & & \\
\hline 9.0 & -0.121902053024897 & -0.001614453434276 & -0.026333197884582 \\
\hline \multirow[t]{2}{*}{10.0} & -0.123437616112352 & -0.014547137896785 & -0.035089753065046 \\
\hline & \multicolumn{3}{|c|}{$V_{0}=1$} \\
\hline 2.0 & 0.682749660131958 & & \\
\hline 3.0 & 0.223625182694464 & & 0.753493962461419 \\
\hline 4.0 & 0.042468064663317 & 0.795108317218819 & 0.398683123449639 \\
\hline 5.0 & -0.038812779897186 & 0.483527207581951 & 0.218493124006869 \\
\hline 7.0 & -0.099869055851552 & 0.183184671173671 & 0.059817320600137 \\
\hline 9.0 & -0.117485345849191 & 0.060959866704373 & -0.001222430190460 \\
\hline \multirow[t]{2}{*}{10.0} & -0.120945131102872 & 0.027546157080191 & -0.017381491379463 \\
\hline & \multicolumn{3}{|c|}{$V_{0}=5$} \\
\hline 1.0 & 4.117395654757679 & & \\
\hline 2.0 & 1.060090806849548 & 4.259590443923497 & 2.354023491802505 \\
\hline 3.0 & 0.337547804108058 & 1.965280728602923 & 1.008417873338432 \\
\hline 4.0 & 0.088668269207450 & 1.037591546680630 & 0.505128085606687 \\
\hline 5.0 & -0.017121556887133 & 0.597303722751802 & 0.272174125945653 \\
\hline 7.0 & -0.093932334169163 & 0.220986935924746 & 0.078075429374547 \\
\hline 9.0 & -0.115667122425525 & 0.077074533332219 & 0.006451568044212 \\
\hline \multirow[t]{2}{*}{10.0} & -0.119934725736816 & 0.038611646265036 & -0.012169285546458 \\
\hline & \multicolumn{3}{|c|}{$V_{0}=10$} \\
\hline 1.0 & 5.080928139664123 & & 9.153308707345341 \\
\hline 2.0 & 1.186465593349953 & 4.872083554044275 & 2.604530792814451 \\
\hline 3.0 & 0.374146997487948 & 2.117592562251291 & 1.082876362415076 \\
\hline 4.0 & 0.102968397805876 & 1.098935329231172 & 0.536078559948866 \\
\hline 5.0 & -0.010583987945514 & 0.627608247099480 & 0.287530094114737 \\
\hline 7.0 & -0.092198533908111 & 0.231170175626802 & 0.083151192402772 \\
\hline 9.0 & -0.115143074788148 & 0.081388712116576 & 0.008543070474369 \\
\hline 10.0 & -0.119644113544775 & 0.041563646553631 & -0.010759126607331 \\
\hline
\end{tabular}

\subsubsection{Entropía de Shannon y corrección de núcleo de tamaño finito}

Una vez obtenidas las funciones de onda del sistema, pueden calcularse diversos valores esperados y propiedades físicas del sistema. Este trabajo está enfocado al cálculo de dos propiedades poco estudiadas en el caso de sistemas cuánticos confinados: 
La entropía informática de Shannon en el espacio de configuraciones, definida como

$$
S_{r}=-\int \rho(\vec{r}) \ln \rho(\vec{r}) d \vec{r}
$$

Donde $\rho(\vec{r})$ es la densidad de probabilidad del electrón. En el caso del átomo de hidrógeno confinado dentro de una cavidad penetrable se tiene

$$
S_{r}=-\int_{r=0}^{r=R_{c}} \rho_{i} \ln \rho_{i} d \vec{r}-\int_{r=R_{c}}^{r=\infty} \rho_{e} \ln \rho_{e} d \vec{r}
$$

Donde

$$
\begin{gathered}
\rho_{i}=\left|\psi_{i}(r, \theta, \varphi)\right|^{2}=\left|R_{i}(r)\right|^{2}\left|Y_{l m}(\theta, \varphi)\right|^{2} \\
\rho_{e}=\left|\psi_{e}(r, \theta, \varphi)\right|^{2}=\left|R_{e}(r)\right|^{2}\left|Y_{l m}(\theta, \varphi)\right|^{2}
\end{gathered}
$$

Son las densidades de probabilidad electrónicas dentro y fuera de la cavidad, respectivamente. En este trabajo se utilizó a la entropía de Shannon como una medida del grado de localización (o deslocalización) del electrón.

Por otro lado, también se puede calcular la corrección a la energía del estado base debida al tamaño finito del núcleo. El hamiltoniano del átomo de hidrógeno confinado, considerando al núcleo como una esfera de radio $r_{0}$ y carga distribuida uniformemente, está dado por

$$
H=-\frac{\nabla^{2}}{2}+V_{r}(r)
$$

Donde

$$
V_{r}(r)= \begin{cases}\frac{1}{2 r_{0}}\left[\left(\frac{r}{r_{0}}\right)^{2}-3\right], & 0 \leq r \leq r_{0} \\ -\frac{1}{r}, & r_{0} \leq r \leq R_{c} \\ V_{0}, & R_{c}<r<\infty\end{cases}
$$

Los dos primeros términos en la ecuación (1.94) aparecen debido al potencial de interacción entre el electrón y el núcleo, dentro y fuera de éste, respectivamente [12, 79, 80, 106]. El tercer término aparece al confinar al átomo dentro de una cavidad esférica de radio $R_{c}$ y altura de la barrera $V_{0}$.

Se define la función 


$$
\tilde{V}(r)=\left\{\begin{aligned}
-\frac{1}{r}, & 0 \leq r \leq r_{0} \\
0, & r>r_{0}
\end{aligned}\right.
$$

Sumando y restando $\tilde{V}(r)$ a la ecuación (1.93) y reagrupando términos se obtiene

$$
H=-\frac{\nabla^{2}}{2}+V(r)+H^{\prime}
$$

Donde $V(r)$ es el potencial dado en la ecuación (1.77). El hamiltoniano (1.96) puede reescribirse en la forma más familiar

$$
H=H^{(0)}+H^{\prime}
$$

De la ecuación (1.96) inmediatamente pueden identificarse los dos primeros términos de la derecha como el hamiltoniano del átomo de hidrógeno confinado dentro de una cavidad esférica penetrable, considerando al núcleo como una partícula puntual, como se estudió en la sección anterior. Este hamiltoniano no perturbado $H^{(0)}$ tiene eigenfunciones $\psi_{n l m}^{(0)}$ dadas por (1.78)

La perturbación $H^{\prime}$ está dada por

$$
H^{\prime}=\left\{\begin{array}{c}
\frac{1}{2 r_{0}}\left[\left(\frac{r}{r_{0}}\right)^{2}-3\right]+\frac{1}{r}, r \leq r_{0} \\
0, r>r_{0}
\end{array}\right.
$$

La corrección a primer orden a la energía está dada por

$$
E_{n l}^{(1)}=\left\langle\psi_{n l m}^{(0)}\left|H^{\prime}\right| \psi_{n l m}^{(0)}\right\rangle
$$

Sustituyendo (1.78) en (1.99) se obtiene

$$
E_{n l}^{(1)}=\frac{1}{2 r_{0}} \int_{0}^{r_{0}}\left|R_{n l}^{(0)}(r)\right|^{2}\left[\left(\frac{r}{r_{0}}\right)^{2}-3+\frac{2 r_{0}}{r}\right] r^{2} d r
$$

En la región $r \leq r_{0}$, dado que la extensión espacial de la función de onda es mucho mayor que la extensión espacial del núcleo, se puede hacer la aproximación

$$
R_{n l}^{(0)}(r) \cong R_{n l}^{(0)}(0)
$$

Dado que las funciones con simetría $S(l=0)$ son las únicas con valores distintos de cero en el origen, estos estados son los únicos en sufrir un corrimiento en los niveles de energía debido al tamaño finito del núcleo. La ecuación (1.100) entonces queda como 


$$
E_{n 0}^{(1)}=\frac{r_{0}^{2}}{10}\left|R_{n l}^{(0)}(0)\right|^{2}
$$

Para los cálculos realizados en este trabajo se utilizó el radio medido más reciente para el núcleo del átomo de hidrógeno, es decir, el radio de un protón, el cual es $r_{0}=0.8335 \mathrm{fm}$ $\left(1.575086726 \times 10^{-5}\right.$ bohrs $)[81]$.

En las Tablas 1.18 a 1.22 se muestran las correcciones a la energía del estado base del AHC, al considerar el núcleo de tamaño finito. Esta corrección es pequeña comparada con la energía no perturbada. Conforme el radio de confinamiento aumenta, la corrección $E_{10}^{(1)}$ tiende al valor de la corrección a primer orden del átomo de hidrógeno libre, $E_{\text {free }}^{(1)}=$ $0.9923592777 \times 10^{-10}$ hartrees, de acuerdo con la ecuación (1.102). Puede observarse que la corrección a la energía depende de $R_{c}$ y $V_{0}$. Este comportamiento puede verse más claramente en la Figura 1.13, donde se grafica la razón entre la corrección a la energía del estado base del AHC debida al núcleo finito, relativa a la corrección en el átomo de hidrógeno libre. Debe notarse que esta razón es independiente del valor de $r_{0}$ y sólo depende de la razón de las funciones de onda evaluadas en el origen

$$
\frac{E_{10}^{(1)}}{E_{\text {free }}^{(1)}}=\frac{\left|R_{n l}^{(0)}(0)\right|^{2}}{\left|R_{\text {free }}^{(0)}(0)\right|^{2}}
$$

Donde $R_{f r e e}^{(0)}(r)$ y $R_{n l}^{(0)}(r)$ son las funciones de onda radiales del átomo de hidrógeno libre y del AHC, respectivamente. 
Tabla 1.18. Corrección a la energía del estado base del AHC debida al núcleo finito, $E_{10}^{(1)}$, para una barrera de altura $V_{0}=0$ hartrees. Razón entre la corrección a la energía del sistema confinado $E_{10}^{(1)}$ y la corrección del átomo de hidrógeno libre $E_{f r e e}^{(1)}$, y entropía de Shannon en el espacio de configuraciones $S_{r}$.

\begin{tabular}{|l|c|c|c|}
\hline$R_{C}$ & $E_{10}^{(1)}\left(10^{-10}\right)$ & $E_{10}^{(1)} / E_{\text {free }}^{(1)}$ & $S_{r}$ \\
\hline 0.73 & 0.097237055681562 & 0.098 & 12.246873690255589 \\
\hline 0.74 & 0.224982152489368 & 0.227 & 9.784575732147416 \\
\hline 0.75 & 0.342781939506244 & 0.345 & 8.556467215203056 \\
\hline 0.76 & 0.451432482538053 & 0.455 & 7.754771071447240 \\
\hline 0.77 & 0.551659500981923 & 0.556 & 7.170265298877441 \\
\hline 0.78 & 0.644125274108404 & 0.649 & 6.717050133854774 \\
\hline 0.79 & 0.729434798685880 & 0.735 & 6.351485406529977 \\
\hline 0.8 & 0.808141286815622 & 0.814 & 6.048388966914264 \\
\hline 0.9 & 1.325196851197549 & 1.335 & 4.514053722419367 \\
\hline 1.0 & 1.544741614031751 & 1.557 & 3.938788997241782 \\
\hline 1.1 & 1.621160850903080 & 1.634 & 3.665975982097357 \\
\hline 1.2 & 1.627437259807232 & 1.640 & 3.529690139716898 \\
\hline 1.3 & 1.599746765336471 & 1.612 & 3.465408517601437 \\
\hline 1.4 & 1.556616580910408 & 1.569 & 3.442443444848674 \\
\hline 1.5 & 1.507712255705773 & 1.519 & 3.444342042038387 \\
\hline 2.0 & 1.287458459987721 & 1.297 & 3.593196462374467 \\
\hline 2.5 & 1.151246460119175 & 1.160 & 3.772103694815810 \\
\hline 3.0 & 1.075285232620169 & 1.084 & 3.911728836412053 \\
\hline 3.5 & 1.034355146835889 & 1.042 & 4.007437127049752 \\
\hline 4.0 & 1.012980250520460 & 1.021 & 4.067874832242422 \\
\hline 4.5 & 1.002187518743767 & 1.010 & 4.103597631944854 \\
\hline 5.0 & 0.996918364180960 & 1.005 & 4.123556199637507 \\
\hline
\end{tabular}


Tabla 1.19. Corrección a la energía del estado base del AHC debida al núcleo finito, $E_{10}^{(1)}$, para una barrera de altura $V_{0}=1$ hartrees. Razón entre la corrección a la energía del sistema confinado $E_{10}^{(1)}$ y la corrección del átomo de hidrógeno libre $E_{f r e e}^{(1)}$, y entropía de Shannon en el espacio de configuraciones $S_{r}$.

\begin{tabular}{|l|c|c|c|}
\hline$R_{c}$ & $E_{10}^{(1)}\left(10^{-10}\right)$ & $E_{10}^{(1)} / E_{\text {free }}^{(1)}$ & $S_{r}$ \\
\hline 0.56 & 0.372352642216478 & 0.375 & 9.331841560806192 \\
\hline 0.57 & 0.718517377342278 & 0.724 & 7.421627512613028 \\
\hline 0.58 & 1.027601736276912 & 1.036 & 6.385560039745352 \\
\hline 0.59 & 1.303515021410208 & 1.314 & 5.694990908736403 \\
\hline 0.6 & 1.549718543214564 & 1.562 & 5.189171118049536 \\
\hline 0.7 & 2.912076839689307 & 2.934 & 3.207882611318868 \\
\hline 0.8 & 3.248513893236404 & 3.274 & 2.669510522086198 \\
\hline 0.9 & 3.210701333716708 & 3.235 & 2.479372191723856 \\
\hline 1.0 & 3.040601954128760 & 3.064 & 2.427532636386198 \\
\hline 1.5 & 2.112672546109264 & 2.129 & 2.711122270986703 \\
\hline 2.0 & 1.594215224261598 & 1.606 & 3.103617128742421 \\
\hline 2.5 & 1.322566029551825 & 1.333 & 3.425803092473663 \\
\hline 3.0 & 1.174237229223259 & 1.183 & 3.668202820221311 \\
\hline 3.5 & 1.091418868357505 & 1.100 & 3.841584619494671 \\
\hline 4.0 & 1.045192760674669 & 1.053 & 3.959801760315439 \\
\hline 4.5 & 1.019814074359305 & 1.028 & 4.036507289422293 \\
\hline 5.0 & 1.006238085817960 & 1.014 & 4.083854043241113 \\
\hline
\end{tabular}


Tabla 1.20. Corrección a la energía del estado base del AHC debida al núcleo finito, $E_{10}^{(1)}$, para una barrera de altura $V_{0}=5$ hartrees. Razón entre la corrección a la energía del sistema confinado $E_{10}^{(1)}$ y la corrección del átomo de hidrógeno libre $E_{f r e e}^{(1)}$, y entropía de Shannon en el espacio de configuraciones $S_{r}$.

\begin{tabular}{|c|c|c|c|}
\hline$R_{C}$ & $E_{10}^{(1)}\left(10^{-10}\right)$ & $E_{10}^{(1)} / E_{\text {free }}^{(1)}$ & $S_{r}$ \\
\hline 0.36 & 0.697116487714404 & 0.702 & 9.393410967935432 \\
\hline 0.37 & 2.567968421088848 & 2.588 & 5.609856413985652 \\
\hline 0.38 & 4.118176960410371 & 4.150 & 4.249418928100824 \\
\hline 0.39 & 5.400014459629074 & 5.442 & 3.460727538474364 \\
\hline 0.4 & 6.456668836007435 & 6.506 & 2.929598321888637 \\
\hline 0.5 & 10.240494963124124 & 10.319 & 1.237192881744071 \\
\hline 0.6 & 9.598999388792230 & 9.673 & 0.986303527028847 \\
\hline 0.7 & 8.251663040869087 & 8.315 & 1.014384582112780 \\
\hline 0.8 & 6.980485974979947 & 7.034 & 1.132704022132080 \\
\hline 0.9 & 5.926377707785826 & 5.972 & 1.283417064852144 \\
\hline 1.0 & 5.081081669583943 & 5.120 & 1.444098375580616 \\
\hline 1.5 & 2.781656875168397 & 2.803 & 2.190728058871032 \\
\hline 2.0 & 1.889596355389960 & 1.904 & 2.764300189009321 \\
\hline 2.5 & 1.473407522212738 & 1.485 & 3.190785540604040 \\
\hline 3.0 & 1.256864293020748 & 1.267 & 3.503945230647304 \\
\hline 3.5 & 1.137865941478808 & 1.147 & 3.728780330345312 \\
\hline 4.0 & 1.071253920964084 & 1.080 & 3.884868529581913 \\
\hline 4.5 & 1.034166451405600 & 1.042 & 3.988777257319570 \\
\hline 5.0 & 1.013927192430064 & 1.022 & 4.054799615123246 \\
\hline
\end{tabular}


Tabla 1.21. Corrección a la energía del estado base del AHC debida al núcleo finito, $E_{10}^{(1)}$, para una barrera de altura $V_{0}=10$ hartrees. Razón entre la corrección a la energía del sistema confinado $E_{10}^{(1)}$ y la corrección del átomo de hidrógeno libre $E_{f r e e}^{(1)}$, y entropía de Shannon en el espacio de configuraciones $S_{r}$.

\begin{tabular}{|l|c|c|c|}
\hline$R_{c}$ & $E_{10}^{(1)}\left(10^{-10}\right)$ & $E_{10}^{(1)} / E_{\text {free }}^{(1)}$ & $S_{r}$ \\
\hline 0.28 & 1.358791994978611 & 1.369 & 8.645390731674327 \\
\hline 0.29 & 6.176219111451923 & 6.224 & 4.263207338736669 \\
\hline 0.3 & 9.930542047590157 & 10.007 & 2.894221820570743 \\
\hline 0.4 & 20.405228083842307 & 20.562 & 0.344486274322369 \\
\hline 0.5 & 17.491618217286550 & 17.626 & 0.184178018580150 \\
\hline 0.6 & 13.822727567461880 & 13.929 & 0.316907838836485 \\
\hline 0.7 & 10.917393851422899 & 11.001 & 0.522238335023892 \\
\hline 0.8 & 8.766306263935839 & 8.834 & 0.743384600643997 \\
\hline 0.9 & 7.179161687379589 & 7.234 & 0.961578933408612 \\
\hline 1.0 & 5.992750426662855 & 6.039 & 1.170138953560959 \\
\hline 1.5 & 3.048420555184699 & 3.072 & 2.037078877345655 \\
\hline 2.0 & 1.999717840968987 & 2.015 & 2.662522202438685 \\
\hline 2.5 & 1.527421154803173 & 1.539 & 3.119478526376661 \\
\hline 3.0 & 1.285799849400771 & 1.296 & 3.453459178195202 \\
\hline 3.5 & 1.153976852962736 & 1.163 & 3.693572995862681 \\
\hline 4.0 & 1.080287038138810 & 1.089 & 3.861073025827234 \\
\hline 4.5 & 1.039165583170746 & 1.047 & 3.973341483370190 \\
\hline 5.0 & 1.016626789889568 & 1.024 & 4.045232737075256 \\
\hline & & & \\
\hline
\end{tabular}

En el caso límite de una cavidad esférica de paredes impenetrables, la corrección a la energía crece rápidamente conforme $R_{c}$ tiende a cero, ya que al reducir $R_{c}$, el electrón se acerca más al núcleo sin la posibilidad de escapar. En la Figura 1.13 puede observarse que para un radio de confinamiento de $R_{c}=1$ bohr, la corrección a la energía $E_{10}^{(1)}$ es 10 veces mayor que en el caso del átomo de hidrógeno libre de confinamiento. 
Tabla 1.22. Corrección a la energía del estado base del AHC debida al núcleo finito, $E_{10}^{(1)}$, para una barrera de altura $V_{0}=\infty$ hartrees. Razón entre la corrección a la energía del sistema confinado $E_{10}^{(1)}$ y la corrección del átomo de hidrógeno libre $E_{\text {free }}^{(1)}$, y entropía de Shannon en el espacio de configuraciones $S_{r}$.

\begin{tabular}{|c|c|c|c|}
\hline$R_{c}$ & $E_{10}^{(1)}\left(10^{-10}\right)$ & $E_{10}^{(1)}\left(R_{c}\right) / E_{\text {free }}^{(1)}$ & $S_{r}$ \\
\hline 0.5 & 54.536609722874516 & 54.957 & -1.470340682989853 \\
\hline 0.6 & 33.740936784485875 & 34.001 & -0.938219378325265 \\
\hline 0.7 & 22.720335291772468 & 22.895 & -0.491174380130971 \\
\hline 0.8 & 16.278628704347120 & 16.404 & -0.106572435383932 \\
\hline 0.9 & 12.229690823293298 & 12.324 & 0.230177356450901 \\
\hline 1.0 & 9.538267646127938 & 9.612 & 0.529030309408580 \\
\hline 1.5 & 3.969102513288022 & 4.000 & 1.649056074996559 \\
\hline 2.0 & 2.355893659642013 & 2.374 & 2.396669173973555 \\
\hline 2.5 & 1.695773616320432 & 1.709 & 2.929199524532576 \\
\hline 3.0 & 1.374211998517624 & 1.385 & 3.316365441502659 \\
\hline 3.5 & 1.202793634385484 & 1.212 & 3.596356649465181 \\
\hline 4.0 & 1.107644656981758 & 1.116 & 3.794245492610194 \\
\hline 4.5 & 1.054376061163177 & 1.062 & 3.929245415029455 \\
\hline 5.0 & 1.024902478009865 & 1.033 & 4.017444189165229 \\
\hline
\end{tabular}




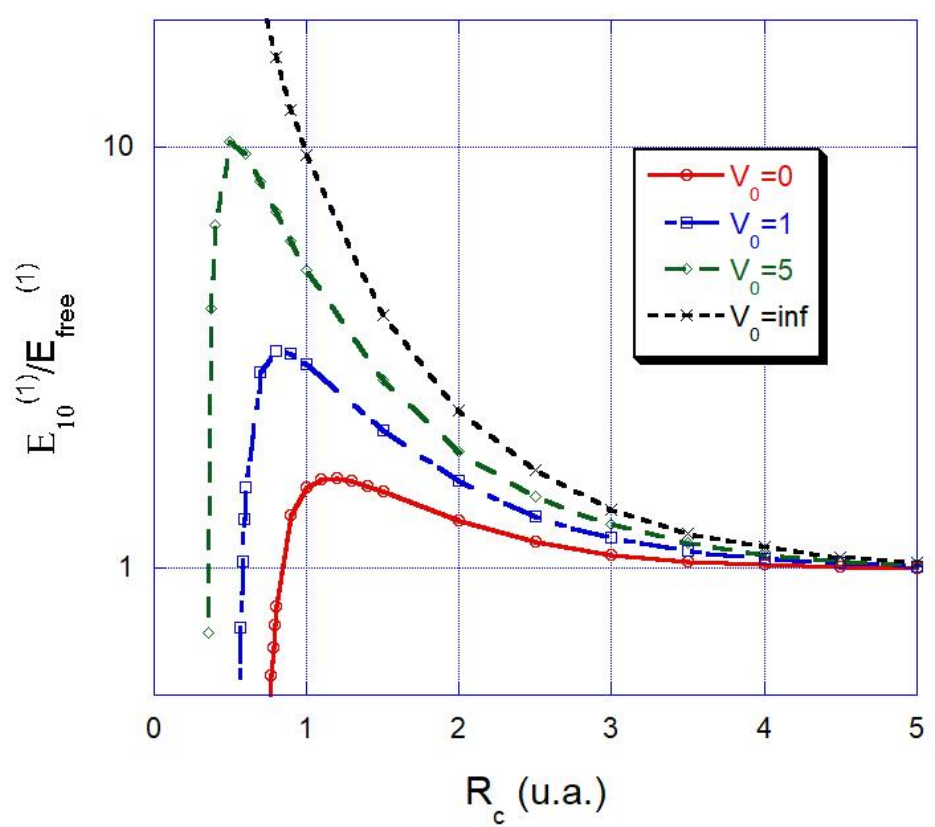

Figura 1.13. Razón $E_{10}^{(1)} / E_{\text {free }}^{(1)}$ de la corrección a la energía del estado base del AHC sobre la corrección del átomo de hidrógeno libre, como función de $R_{c}$.

Estos valores para barreras impenetrables están en completo acuerdo con los calculados por Goldman y Joslin [12]. Ellos encontraron que para valores muy pequeños de $R_{c}$ y estados muy excitados, la corrección a la energía $E_{10}^{(1)}$, puede ser varios órdenes de magnitud mayor que para el átomo de hidrógeno libre de confinamiento.

Para un valor finito de la altura de la barrera $V_{0}$, la situación es diferente. La corrección a la energía $E_{10}^{(1)}$ crece conforme $R_{c}$ decrece, y alcanza su valor máximo a cierto radio de confinamiento $R_{\max }$. Si $R_{c}$ continúa decreciendo, la corrección a la energía decrece también, aproximándose a cero cuando $R_{c}$ se acerca al radio crítico en el cual la energía total del electrón es igual a la altura de la barrera $V_{0}$.

Intuitivamente, se espera que la corrección $E_{10}^{(1)}$ sea mayor para una cavidad de tamaño pequeño, dentro de la cual el electrón se encuentra más cerca del núcleo, esto es, para una cavidad en la cual la densidad electrónica sea más compacta. Una manera de cuantificar qué 
tan compacta se encuentra la densidad electrónica es por medio de la entropía de Shannon [42, 96-99]. Esta idea está cimentada en la interpretación de la localización-deslocalización de las partículas asociada con la entropía de Shannon $S_{r}$. Un valor pequeño de $S_{r}$ significa que la densidad electrónica se encuentra más localizada alrededor del núcleo [42]. En $R_{c}^{*}$, donde la curva de la entropía como función del radio de confinamiento tiene un valor mínimo, la densidad electrónica es más compacta alrededor del núcleo, y por tanto la corrección a la energía debida al núcleo finito $E_{10}^{(1)}$ es mayor. En las Figuras 1.14 a 1.17 se grafican la corrección $E_{10}^{(1)}$ y la entropía de Shannon $S_{r}$ para algunos valores de $V_{0}$, como funciones de $R_{c}$. En la Figura 1.14 se grafican $E_{10}^{(1)}$ y $S_{r}$ para una barrera de altura $V_{0}=\infty$. Se observa que cuando $R_{c}$ decrece, $S_{r}$ también decrece. Esto significa que la densidad electrónica alrededor del núcleo aumenta y hay un incremento en $E_{10}^{(1)}$. Este comportamiento continúa conforme $R_{C}$ disminuye.

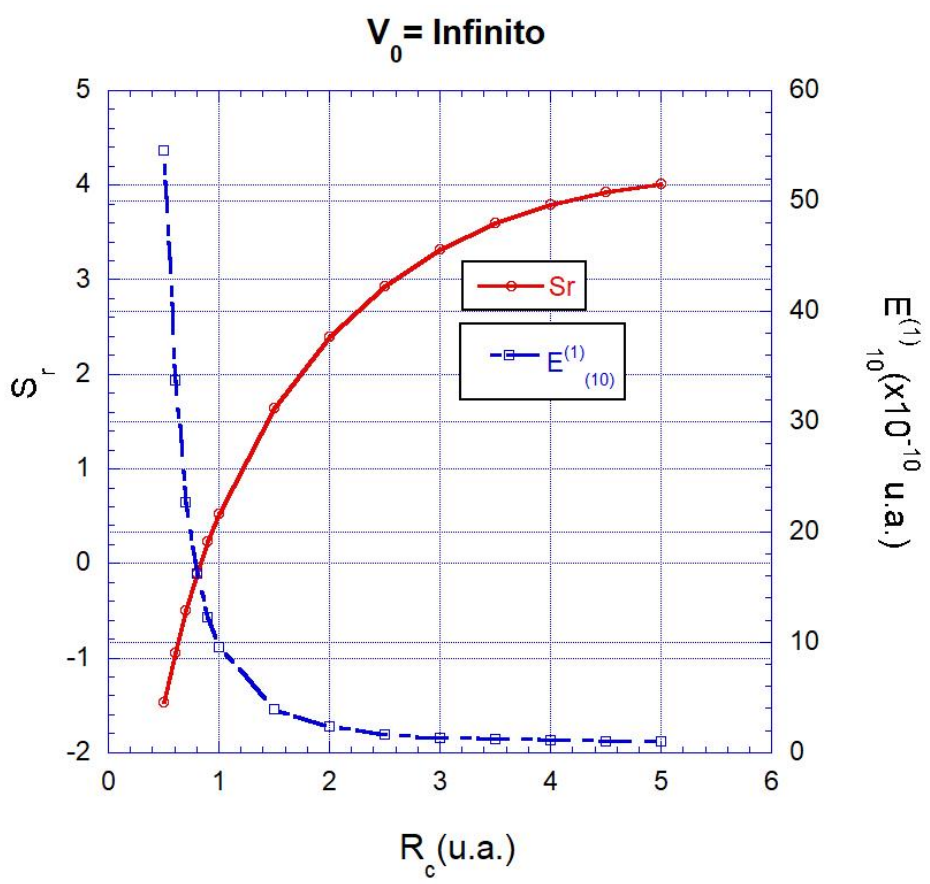

Figura 1.14. Corrección de núcleo finito $E_{10}^{(1)}$ y entropía de Shannon $S_{r}$ como funciones del radio de confinamiento $R_{c}$, para una barrera esférica impenetrable. 
En las Figuras 1.15 a 1.17 se muestran la corrección de núcleo finito $E_{10}^{(1)}$ y la entropía de Shannon $S_{r}$ para valores finitos de $V_{0}$. En todas las figuras, se observa que la curva de la corrección a la energía $E_{10}^{(1)}$ alcanza su valor máximo a un radio de confinamiento $R_{\text {max }}$, el cual depende del valor de $V_{0}$. Se puede ver también que la curva de la entropía de Shannon $S_{r}$ alcanza su valor mínimo a un radio de confinamiento $R_{c}^{*}$, esto es, dentro de una caja esférica de radio $R_{c}^{*}$, la densidad electrónica es más compacta alrededor del núcleo. De las Figuras 1.15 a 1.17 se puede observar que $R_{\max }<R_{c}^{*}$. Este resultado puede interpretarse de la siguiente manera: conforme $R_{c}$ disminuye, $S_{r}$ también disminuye, y la densidad electrónica se vuelve más compacta hasta alcanzar un valor máximo en $R_{c}^{*}$. Conforme $R_{c}$ continúa decreciendo, $S_{r}$ empieza a aumentar y el electrón comienza a deslocalizarse. Por otro lado, como se muestra en la Figura 1.18, la función de onda aumenta su valor en el origen y dentro de la barrera. Si el radio de confinamiento continúa disminuyendo, el valor de la función de onda en el origen decae rápidamente. Este proceso continúa hasta que la ionización toma lugar.

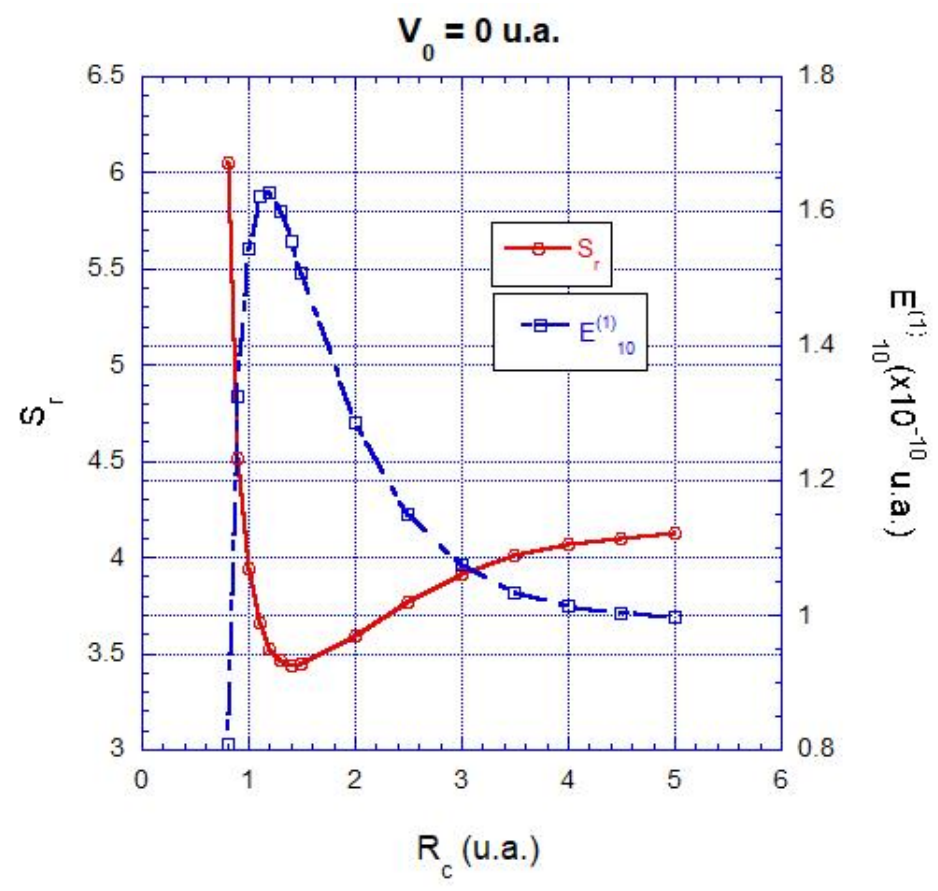

Figura 1.15. Corrección de núcleo finito $E_{10}^{(1)}$ y entropía de Shannon $S_{r}$ como funciones del radio de confinamiento $R_{c}$, para $V_{0}=0$ hartrees. 


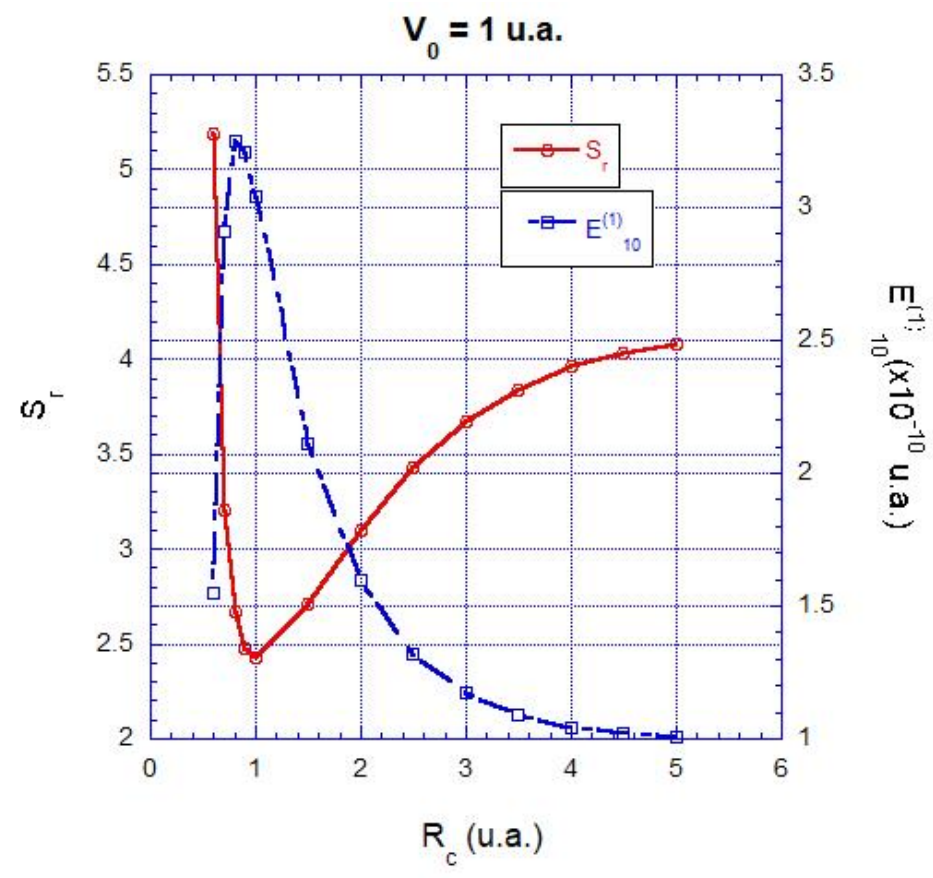

Figura 1.16. Corrección de núcleo finito $E_{10}^{(1)}$ y entropía de Shannon $S_{r}$ como funciones del radio de confinamiento $R_{c}$, para $V_{0}=1$ hartrees.

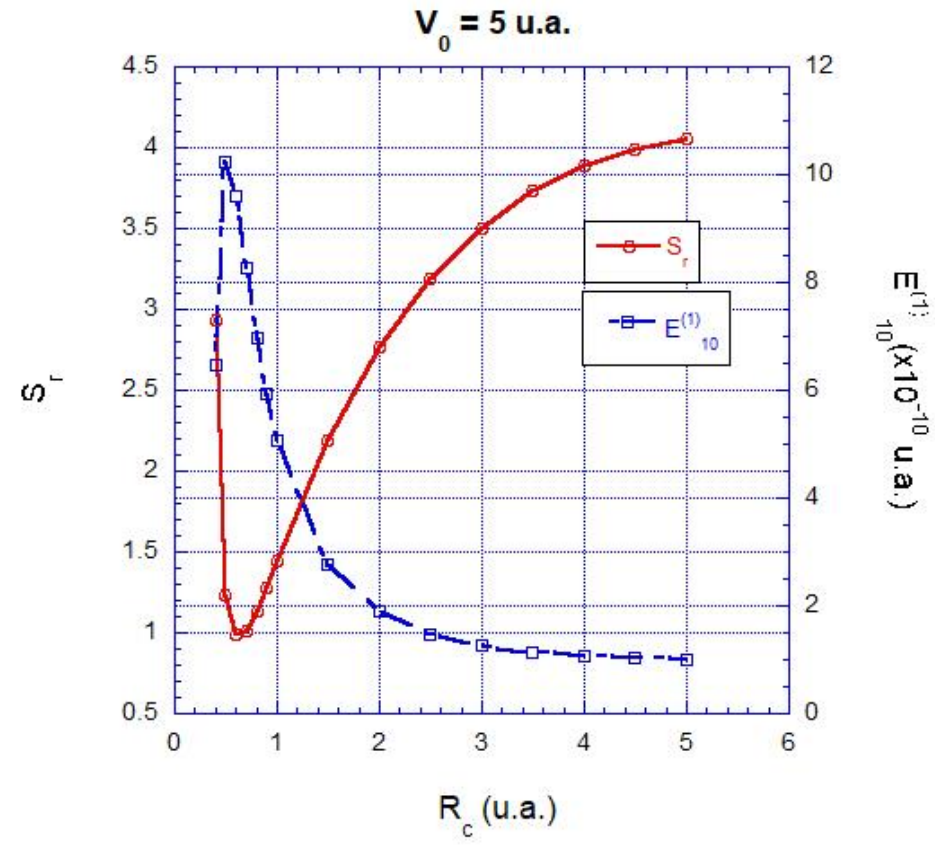

Figura 1.17. Corrección de núcleo finito $E_{10}^{(1)}$ y entropía de Shannon $S_{r}$ como funciones del radio de confinamiento $R_{c}$, para $V_{0}=5$ hartrees. 


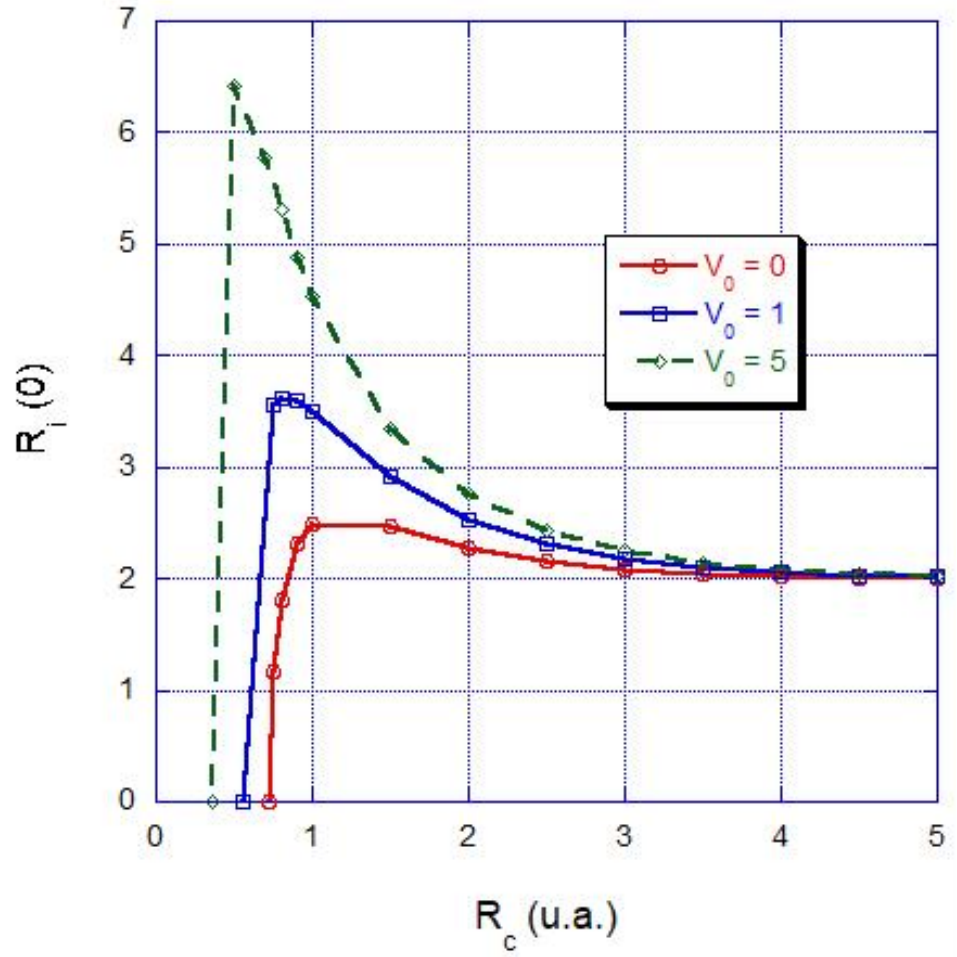

Figura 1.18. Función de onda radial dentro de la barrera, evaluada en el origen, como función del radio de confinamiento y la altura de la barrera.

\subsection{Conclusiones}

Se resolvió el problema del átomo de hidrógeno confinado dentro de una barrera esférica de paredes impenetrables usando el método variacional de Rayleigh-Ritz, tomando como función de prueba la función de onda del estado base del átomo de hidrógeno libre de confinamiento, multiplicada por una función de corte. Se usó un solo parámetro variacional. Se usaron diferentes funciones de corte para los cálculos, encontrando que la precisión en éstos depende del radio de confinamiento y de la función de corte utilizada. Los resultados obtenidos para la energía del estado base del AHC difieren del valor exacto en menos del $1 \%$ en la mayoría de los casos, con algunas variaciones principalmente para radios de confinamiento pequeños. Dependiendo de la región de confinamiento que se esté estudiando, es conveniente usar una función de corte en lugar de otra, ya que se pueden obtener resultados ligeramente mejores; sin embargo, por su simplicidad y la precisión obtenida, la función de corte recomendada es de la forma $1-\left(r / R_{c}\right)^{n}$, donde $n$ es un entero positivo. En este trabajo 
se realizaron cálculos con $n=1,2,3$ y 4, y los mejores resultados se obtuvieron con $n=2$, en la región $R_{c} \leq 1.7$ bohrs, y para $n=4$, con $R_{c}>2$ bohrs. Para $n=1$, se obtiene el valor exacto de la energía con un confinamiento de $R_{c}=2$ bohrs.

Aun así, funciones de corte que incluyen funciones esféricas de Bessel en general dan buenos resultados, especialmente en la región de confinamiento fuerte, además son muy estables y eficientes para realizar cálculos computacionales, por lo que en capítulos posteriores se usarán funciones de este tipo para el estudio de sistemas confinados más complejos. [46]

La entropía de Shannon en el espacio de coordenadas muestra un comportamiento creciente a medida que aumenta el radio de confinamiento. El que una función de prueba tenga la energía más baja de entre un conjunto de funciones de onda no implica que su entropía de Shannon será la más alta. Sin embargo, el criterio de la entropía de Shannon puede ser importante en situaciones en las que se está interesado en la calidad de la densidad electrónica, ya que en trabajos realizados por otros autores se ha demostrado que algunas propiedades calculadas a partir de una función de onda, no necesariamente dan buenos resultados sólo porque para la energía sí los sean [96, 97].

Los resultados obtenidos tanto con barreras impenetrables como penetrables muestran la utilidad del método variacional directo en el estudio de sistemas cuánticos en general, y en sistemas confinados en particular.

El comportamiento de las propiedades físicas del átomo de hidrógeno confinado por barreras penetrables es diferente al caso de barreras impenetrables, esto es más evidente para valores pequeños de $V_{0}$.

Los sistemas cuánticos confinados dentro de paredes suaves son más realistas y flexibles que los modelos con paredes duras, ya que permiten la descripción de las fuerzas de atracción de van der Waals producidas por las partículas vecinas. Esto se logra con una elección apropiada de la altura de la barrera.

La función de onda propuesta en la Sección 1.1.2 es muy parecida a la de una partícula libre dentro de una caja esférica impenetrable, y se incluye un término exponencial para tomar en cuenta la interacción coulombiana entre el núcleo y el electrón en la región dentro de la 
esfera. De esta forma, las energías obtenidas muestran una mejora, especialmente para radios de confinamiento pequeños y alturas de la barrera grandes.

Todos los cálculos numéricos variacionales fueron realizados en Mathematica 9, y no presentan problemas de convergencia, siempre que se considere $\beta>0$. Por otra parte, las funciones de onda propuestas por Marín y Cruz [29] proporcionan un funcional de la energía que puede presentar problemas para converger al encontrar el valor mínimo de la energía, por lo que resulta difícil automatizar el proceso.

La función de prueba usada en la Sección 1.1.2 se construyó para representar adecuadamente al átomo hidrogenoide en confinamientos fuertes, es decir, para radios $R_{c}<2$ u.a.

Se hallaron las energías y funciones de onda exactas del átomo de hidrógeno confinado dentro de una esfera impenetrable, siguiendo la metodología llevada a cabo por Aquino et al. [35]. Con estas funciones de onda, se estudió el espectro de estructura fina del AHC.

En este sentido, los estados de una misma capa están degenerados en el caso del átomo de hidrógeno libre. Esta degeneración es parcialmente removida debido al confinamiento espacial, donde los estados con el menor momento angular $l$ son los de más alta energía.

Aunque la energía cinética del átomo aumenta debido al confinamiento, estos valores permanecen dentro del rango de la mecánica cuántica no relativista. Sin embargo, los efectos relativistas, aunque pequeños, dan lugar a importantes correcciones a la estructura fina, los cuales deberían ser fáciles de detectar experimentalmente, ya que son algunos órdenes de magnitud más altos que en el caso del átomo de hidrógeno libre.

La estructura fina aparece debido a tres diferentes mecanismos: (1) una corrección relativista a la energía cinética del electrón, que se hace más negativa a medida que el sistema se encuentra más confinado. Este efecto relativista es suficientemente pequeño para ser tratado como una corrección aplicando mecánica cuántica no relativista. (2) Un término de acoplamiento espín-órbita debido a la interacción entre el momento angular orbital y el momento de espín del electrón. Este término divide los niveles de energía para una $l$ dada, las energías de estos nuevos estados están determinadas por el número cuántico principal $n$ y el momento angular total $j$. Conforme el confinamiento del átomo aumenta, la brecha entre estos estados se vuelve más grande. Para estados con los mismos valores de $n$ y $l$, el estado 
con el valor más bajo de $j$ es el que tiene la corrección de acoplamiento espín-órbita más baja. (3) El término de Darwin, el cual es una corrección relativista a la energía potencial y actúa sólo en el origen, por lo que sólo afecta a los estados con simetría $S(l=0)$, e incrementa la energía de dichos estados conforme el confinamiento ejercido sobre el átomo se hace más fuerte.

En otras palabras, la estructura fina del AHC se vuelve más importante conforme la presión ejercida sobre el átomo aumenta. Sin embargo, estas correcciones de estructura fina son pequeñas en comparación a los eigenvalores de la energía no perturbados, por lo que pueden ser tratadas como pequeñas perturbaciones y la aplicación de teoría de perturbaciones es válida.

Por último, se calcularon las energías y las funciones de onda, con alta precisión numérica, del estado base y de algunos estados excitados del átomo de hidrógeno confinado dentro de una cavidad esférica de paredes penetrables. Con estas funciones de onda obtenidas se pudo calcular la corrección a la energía debida al núcleo de tamaño finito del AHC como función de $R_{c}$ y $V_{0}$. Para barreras finitas, las curvas de la corrección de núcleo finito alcanzan un valor máximo, mientras que las curvas de la entropía de Shannon como función del radio de confinamiento alcanzan un valor mínimo. De acuerdo a estos resultados, el máximo de la corrección de núcleo finito siempre se encuentra cercano a la posición en la cual la entropía de Shannon tiene su valor mínimo, esto es, a la posición en la cual la densidad electrónica se encuentra más compacta alrededor del núcleo. 


\section{Capítulo 2}

\section{Átomo de helio confinado}

\subsection{Solución por el método variacional de Rayleigh-Ritz}

La importancia del átomo de helio radica en que es el sistema multielectrónico más sencillo, y provee un reto importante para la generalización de los sistemas de muchos electrones ya que, a diferencia del hidrógeno, en el helio se puede estudiar la correlación electrónica, la cual es una propiedad esencial de la estructura electrónica de átomos y moléculas. Además, tanto para sistemas libres de confinamiento como para sistemas confinados, debido a la interacción coulombiana entre electrones, la ecuación de Schrödinger no puede resolverse usando separación de variables, por lo que deben utilizarse aproximaciones. La teoría variacional ofrece un acercamiento poderoso y sistemático para la solución aproximada de este tipo de problemas.

En esta sección se calcula la energía del estado base del átomo de helio confinado en función de los parámetros de confinamiento, usando el método variacional directo, siguiendo la metodología llevada a cabo por Marín y Cruz [29], quienes usan como función de prueba el producto de funciones orbitales $1 s$ dadas para cada electrón, multiplicadas por una función que se ajuste adecuadamente a las condiciones de frontera del sistema. Este mismo método es utilizado por Díaz-García y Cruz [62], con más parámetros variacionales que en el caso anterior. Además, para el caso de confinamiento dentro de barreras impenetrables usan una función de prueba correlacionada en las coordenadas radiales. Otros métodos utilizados para hallar la energía de sistemas atómicos confinados de dos electrones han sido Hartree-Fock [69] y el método de mallas de Lagrange [74]. 
Como se vio en la Sección 1.1, funciones de prueba que incluyan funciones esféricas de Bessel dan buenos resultados en la región de confinamiento fuerte; no sólo eso, sino que además este tipo de funciones son más estables para realizar cálculos numéricos, por lo que fueron las que se utilizaron en este trabajo para la obtención de las energías del átomo de helio, como se muestra a continuación.

El modelo utilizado en este trabajo fue propuesto primeramente por Gorecki y Byers-Brown [26]. El hamiltoniano de un átomo helioide confinado, cuyo núcleo se encuentra fijo en el centro de una cavidad esférica de paredes penetrables de radio $R_{c}$, está dado por

$$
H=\frac{\vec{p}_{1}^{2}}{2}+\frac{\vec{p}_{2}^{2}}{2}+V\left(\vec{r}_{1}, \vec{r}_{2}\right)
$$

Donde los dos primeros términos del lado derecho son las energías cinéticas de los electrones, $\mathrm{y}$

$$
V\left(\vec{r}_{1}, \vec{r}_{2}\right)=\left\{\begin{aligned}
-\frac{Z}{r_{1}}-\frac{Z}{r_{2}}+\frac{1}{\left|\vec{r}_{1}-\vec{r}_{2}\right|}, & r_{1}, r_{2} \leq R_{c} \\
V_{0}, & r_{1}, r_{2}>R_{c}
\end{aligned}\right.
$$

Donde $\vec{r}_{1}$ y $\vec{r}_{2}$ denotan las coordenadas de ambos electrones. Dentro de la barrera $\left(r_{1}, r_{2} \leq R_{c}\right)$ el potencial está dado por la interacción coulombiana entre cada electrón y el núcleo, y la interacción entre los electrones. Fuera de la barrera, el potencial está dado simplemente por la altura de la barrera de valor constante $V_{0}$.

La función de prueba utilizada en este trabajo está dada por un producto de funciones tipo hidrógeno confinado (ec. (1.33))

$$
\psi_{i}\left(r_{1}, r_{2}\right)=A j_{0}\left(\frac{\gamma \pi r_{1}}{R_{c}}\right) e^{-\alpha r_{1}} j_{0}\left(\frac{\gamma \pi r_{2}}{R_{C}}\right) e^{-\alpha r_{2}}=A \phi_{i}\left(r_{1}\right) \phi_{i}\left(r_{2}\right)
$$

Para la región $r_{1}, r_{2} \leq R_{c}$. Donde $A$ es una constante de normalización y

$$
\phi_{i}\left(r_{j}\right)=j_{0}\left(\frac{\gamma \pi r_{j}}{R_{c}}\right) e^{-\alpha r_{j}}
$$

$\alpha$ y $\gamma$ son parámetros variacionales.

Para la región exterior $\left(r_{1}, r_{2}>R_{c}\right)$, la función de prueba es 


$$
\psi_{e}\left(r_{1}, r_{2}\right)=B \frac{e^{-\beta r_{1}}}{r_{1}} \frac{e^{-\beta r_{2}}}{r_{2}}=B \phi_{e}\left(r_{1}\right) \phi_{e}\left(r_{2}\right)
$$

$B$ es otra constante de normalización, $\beta$ es un parámetro variacional y

$$
\phi_{e}\left(r_{j}\right)=\frac{e^{-\beta r_{j}}}{r_{j}}
$$

La función de onda debe ser continua en $r_{1}, r_{2}=R_{c}$. De esta condición se obtiene una relación entre las constantes de normalización

$$
B=A R_{c}^{2}\left[j_{0}(\gamma \pi)\right]^{2} e^{2(\beta-\alpha) R_{c}}
$$

De igual forma, la derivada logarítmica debe ser continua en todo el espacio, en particular en $r_{1}, r_{2}=R_{c}$, de donde se obtiene

$$
\beta=-\frac{1}{R_{c}}+\alpha+\frac{\gamma \pi}{R_{c}} \frac{j_{1}(\gamma \pi)}{j_{0}(\gamma \pi)}
$$

Por lo que sólo los parámetros variacionales $\alpha$ y $\gamma$ son independientes.

Los dominios de integración son $\Gamma_{i}$, con ambos electrones dentro del potencial de confinamiento, y $\Gamma_{e}$, con ambos electrones fuera de la cavidad. Nótese que este modelo (ec. (2.2)) considera a ambos electrones ya sea dentro de la barrera, o ambos fuera de ésta. Por simplicidad, no se considera la situación en la que un electrón pueda encontrarse dentro de la cavidad y el otro fuera. De esta forma, hay que calcular la integral de normalización

$$
\begin{aligned}
\langle\psi \mid \psi\rangle=\int_{r_{1}=0}^{r_{1}=R_{c}} & r_{1}^{2} d r_{1} \int_{r_{2}=0}^{r_{2}=R_{c}} r_{2}^{2} d r_{2}\left|\psi_{i}\left(r_{1}, r_{2}\right)\right|^{2} \\
& \quad+\int_{r_{1}=R_{c}}^{r_{1}=\infty} r_{1}^{2} d r_{1} \int_{r_{2}=R_{c}}^{r_{2}=\infty} r_{2}^{2} d r_{2}\left|\psi_{e}\left(r_{1}, r_{2}\right)\right|^{2} \\
= & A^{2} \int_{r_{1}=0}^{r_{1}=R_{c}} r_{1}^{2} d r_{1}\left[j_{0}\left(\frac{\gamma \pi r_{1}}{R_{c}}\right)\right]^{2} e^{-2 \alpha r_{1}} \int_{r_{2}=0}^{r_{2}=R_{c}} r_{2}^{2} d r_{2}\left[j_{0}\left(\frac{\gamma \pi r_{2}}{R_{c}}\right)\right]^{2} e^{-2 \alpha r_{2}} \\
& +B^{2} \int_{r_{1}=R_{c}}^{r_{1}=\infty} r_{1}^{2} d r_{1} \frac{e^{-2 \beta r_{1}}}{r_{1}} \int_{r_{2}=R_{c}}^{r_{2}=\infty} r_{2}^{2} d r_{2} \frac{e^{-2 \beta r_{2}}}{r_{2}}
\end{aligned}
$$

Dado que la variable de integración es muda, podemos tomar $r_{1}=r_{2}=r$. Por tanto

$$
\langle\psi \mid \psi\rangle=A^{2}\left\{\int_{0}^{R_{c}} r^{2} d r\left[j_{0}\left(\frac{\gamma \pi r}{R_{c}}\right)\right]^{2} e^{-2 \alpha r}\right\}^{2}+B^{2}\left(\frac{e^{-2 \beta R_{c}}}{2 \beta}\right)^{2}
$$


Igualando a 1 la ecuación (2.10) y usando la relación (2.7), se puede hallar el valor de la constante de normalización $A$.

El valor esperado de la energía cinética del primer electrón

$$
\begin{aligned}
& \left\langle\psi\left|T_{1}\right| \psi\right\rangle \\
& =\int_{r_{1}=0}^{r_{1}=R_{c}} r_{1}^{2} d r_{1} \int_{r_{2}=0}^{r_{2}=R_{c}} r_{2}^{2} d r_{2} \psi_{i}^{*}\left(r_{1}, r_{2}\right)\left[-\frac{1}{2 r_{1}^{2}} \frac{d}{d r_{1}}\left(r_{1}^{2} \frac{d}{d r_{1}} \psi_{i}\left(r_{1}, r_{2}\right)\right)\right] \\
& +\int_{r_{1}=R_{c}}^{r_{1}=\infty} r_{1}^{2} d r_{1} \int_{r_{2}=R_{c}}^{r_{2}=\infty} r_{2}^{2} d r_{2} \psi_{e}^{*}\left(r_{1}, r_{2}\right)\left[-\frac{1}{2 r_{1}^{2}} \frac{d}{d r_{1}}\left(r_{1}^{2} \frac{d}{d r_{1}} \psi_{e}\left(r_{1}, r_{2}\right)\right)\right] \\
& =-\frac{1}{2} A^{2} \int_{r_{1}=0}^{r_{1}=R_{c}} d r_{1} \phi_{i}^{*}\left(r_{1}\right) \frac{d}{d r_{1}}\left(r_{1}^{2} \frac{d}{d r_{1}} \phi_{i}\left(r_{1}\right)\right) \int_{r_{2}=0}^{r_{2}=R_{c}} r_{2}^{2} d r_{2}\left|\phi_{i}\left(r_{2}\right)\right|^{2} \\
& -\frac{B^{2} e^{-4 \beta R_{c}}}{8}
\end{aligned}
$$

La función de onda radial propuesta es simétrica ante el intercambio de electrones, por lo que de igual forma, para el segundo electrón se tiene

$$
\begin{gathered}
\left\langle\psi\left|T_{2}\right| \psi\right\rangle=-\frac{1}{2} A^{2} \int_{r_{1}=0}^{r_{1}=R_{c}} r_{1}^{2} d r_{1}\left|\phi_{i}\left(r_{1}\right)\right|^{2} \int_{r_{2}=0}^{r_{2}=R_{c}} d r_{2} \phi_{i}^{*}\left(r_{2}\right) \frac{d}{d r_{2}}\left(r_{2}^{2} \frac{d}{d r_{2}} \phi_{i}\left(r_{2}\right)\right) \\
-\frac{B^{2} e^{-4 \beta R_{c}}}{8}
\end{gathered}
$$

El valor esperado de la energía potencial del sistema

$$
\begin{aligned}
\langle\psi|V| \psi\rangle=\int_{r_{1}=0}^{r_{1}=R_{c}} r_{1}^{2} d r_{1} \int_{r_{2}=0}^{r_{2}=R_{c}} r_{2}^{2} d r_{2} \psi_{i}^{*}\left(r_{1}, r_{2}\right)\left(-\frac{Z}{r_{1}}-\frac{Z}{r_{2}}\right. \\
\left.\quad+\frac{1}{\left|\vec{r}_{1}-\vec{r}_{2}\right|}\right) \psi_{i}\left(r_{1}, r_{2}\right)+\int_{r_{1}=R_{c}}^{r_{1}=\infty} r_{1}^{2} d r_{1} \int_{r_{2}=R_{c}}^{r_{2}=\infty} r_{2}^{2} d r_{2}\left|\psi_{e}\left(r_{1}, r_{2}\right)\right|^{2} V_{0} \\
=-2 A^{2} Z \int_{r=0}^{r=R_{c}} d r\left[j_{0}\left(\frac{\gamma \pi r}{R_{c}}\right)\right]^{2} e^{-2 \alpha r} r \int_{r=0}^{r=R_{c}} d r\left[j_{0}\left(\frac{\gamma \pi r}{R_{c}}\right)\right]^{2} e^{-2 \alpha r} r^{2} \\
+A^{2} \int_{r_{1}=0}^{r_{1}=R_{c}} r_{1}^{2} d r_{1} \int_{r_{2}=0}^{r_{2}=R_{c}} r_{2}^{2} d r_{2}\left|\phi_{i}\left(r_{1}\right)\right|^{2}\left|\phi_{i}\left(r_{2}\right)\right|^{2} \frac{1}{\left|\vec{r}_{1}-\vec{r}_{2}\right|} \\
+B^{2} V_{0}\left(\frac{e^{-2 \beta R_{c}}}{2 \beta}\right)^{2}
\end{aligned}
$$

Nótese que en (2.13) aparece la integral 


$$
I=\int d \vec{r}_{1} \int d \vec{r}_{2}\left|\phi_{i}\left(r_{1}\right)\right|^{2}\left|\phi_{i}\left(r_{2}\right)\right|^{2} \frac{1}{\left|\vec{r}_{1}-\vec{r}_{2}\right|}
$$

Para resolverla, se usa el desarrollo [110]

$$
\frac{1}{\left|\vec{r}_{1}-\vec{r}_{2}\right|}=\sum_{l=0}^{\infty} \sum_{m=-l}^{l} \frac{4 \pi}{2 l+1} \frac{r_{<}^{l}}{r_{>}^{l+1}} Y_{l m}^{*}\left(\theta_{1}, \varphi_{1}\right) Y_{l m}\left(\theta_{2}, \varphi_{2}\right)
$$

Entonces

$$
\begin{aligned}
I=\sum_{l=0}^{\infty} \sum_{m=-l}^{l} \frac{4 \pi}{2 l} & +1 \int_{r_{1}=0}^{r_{1}=R_{c}} r_{1}^{2} d r_{1} \int_{r_{2}=0}^{r_{2}=R_{c}} r_{2}^{2} d r_{2}\left|\phi_{i}\left(r_{1}\right)\right|^{2}\left|\phi_{i}\left(r_{2}\right)\right|^{2} \frac{r_{<}^{l}}{r_{>}^{l+1}} \times \\
& \times \int d \Omega_{1} Y_{l m}^{*}\left(\theta_{1}, \varphi_{1}\right) \int d \Omega_{2} Y_{l m}\left(\theta_{2}, \varphi_{2}\right)
\end{aligned}
$$

Multiplicando y dividiendo por $4 \pi$

$$
\begin{aligned}
I= & \sum_{l=0}^{\infty} \sum_{m=-l}^{l} \frac{16 \pi^{2}}{2 l+1} \int_{r_{1}=0}^{r_{1}=R_{c}} r_{1}^{2} d r_{1} \int_{r_{2}=0}^{r_{2}=R_{c}} r_{2}^{2} d r_{2}\left|\phi_{i}\left(r_{1}\right)\right|^{2}\left|\phi_{i}\left(r_{2}\right)\right|^{2} \frac{r_{<}^{l}}{r_{>}^{l+1}} \times \\
& \times \int d \Omega_{1} Y_{l m}^{*}\left(\theta_{1}, \varphi_{1}\right) Y_{00}\left(\theta_{1}, \varphi_{1}\right) \int d \Omega_{2} Y_{00}^{*}\left(\theta_{2}, \varphi_{2}\right) Y_{l m}\left(\theta_{2}, \varphi_{2}\right)
\end{aligned}
$$

Donde $Y_{00}=Y_{00}^{*}=1 / \sqrt{4 \pi}$. Aprovechando la ortonormalidad de los armónicos esféricos

$$
\begin{array}{r}
I=16 \pi^{2} \int_{r_{1}=0}^{r_{1}=R_{c}} r_{1}^{2} d r_{1}\left|\phi_{i}\left(r_{1}\right)\right|^{2} \int_{r_{2}=0}^{r_{2}=R_{c}} r_{2}^{2} d r_{2}\left|\phi_{i}\left(r_{2}\right)\right|^{2} \frac{1}{r_{>}} \\
=16 \pi^{2} \int_{r_{1}=0}^{r_{1}=R_{c}} r_{1}^{2} d r_{1}\left|\phi_{i}\left(r_{1}\right)\right|^{2}\left[\int_{r_{2}=0}^{r_{2}=r_{1}} r_{2}^{2} d r_{2}\left|\phi_{i}\left(r_{2}\right)\right|^{2} \frac{1}{r_{1}}\right. \\
\left.\quad+\int_{r_{2}=r_{1}}^{r_{2}=R_{c}} r_{2}^{2} d r_{2}\left|\phi_{i}\left(r_{2}\right)\right|^{2} \frac{1}{r_{2}}\right]
\end{array}
$$

Las integrales en la región fuera de la esfera se pueden resolver analíticamente de manera sencilla. Por otro lado, en la región dentro de la esfera, las integrales son un poco más complicadas de resolver de forma analítica, debido a la inclusión de la función esférica de Bessel. Sin embargo, en este trabajo se resolvieron con ayuda del programa Mathematica.

En resumen, para hallar el valor de la energía del estado base del sistema, el funcional de la energía debe minimizarse respecto a los parámetros variacionales $\alpha$ y $\gamma$, y está dado por 


$$
E(\alpha, \gamma)=\frac{\left\langle T_{1}\right\rangle+\left\langle T_{2}\right\rangle+\langle V\rangle}{\langle\psi \mid \psi\rangle}
$$

Para que la solución sea físicamente aceptable, la función de onda debe tender a cero cuando $r_{1}, r_{2} \rightarrow \infty$. Esto hace que el parámetro $\beta$, que depende de los valores de $\alpha$ y $\gamma$ de acuerdo con (2.8), esté restringido a sólo tomar valores positivos, es decir, $\beta>0$.

En el caso de barreras impenetrables $\left(V_{0}=\infty\right)$, la función de onda fuera de la esfera toma el valor $\psi_{e}=0$ y el parámetro variacional $\gamma=1$, de tal manera que la función de prueba cumpla con la condición de frontera de Dirichlet $\psi\left(R_{c}\right)=0$. De esta forma, el funcional de la energía (ecuación (2.19)) debe minimizarse respecto al único parámetro variacional disponible, $\alpha$.

En la Tabla 2.1 se muestra la energía del estado base del átomo de helio confinado, como función del radio de confinamiento y la altura de la barrera, usando la función de prueba descrita anteriormente. Se comparan las energías calculadas en este trabajo con las obtenidas por Marín y Cruz [29], por Díaz-García [71] y por Rodríguez-Bautista et al. [69]. En el caso de Marín y Cruz, la función de onda propuesta es de la forma

$$
\begin{gathered}
\psi_{i}=A\left(R_{c}-\alpha r_{1}\right)\left(R_{c}-\alpha r_{2}\right) e^{-\alpha\left(r_{1}+r_{2}\right)}, \quad r_{1}, r_{2} \leq R_{c} \\
\psi_{e}=B \frac{e^{-\beta\left(r_{1}+r_{2}\right)}}{r_{1} r_{2}}, \quad r_{1}, r_{2}>R_{c}
\end{gathered}
$$

Donde $A$ y $B$ son constantes de normalización y $\alpha$ y $\beta$ son parámetros variacionales. Por otro lado, Díaz-García utiliza la misma función de prueba de Marín y Cruz, incluyendo un parámetro variacional extra $\gamma$

$$
\begin{gathered}
\psi_{i}=A\left(R_{c}-\gamma r_{1}\right)\left(R_{c}-\gamma r_{2}\right) e^{-\alpha\left(r_{1}+r_{2}\right)}, \quad r_{1}, r_{2} \leq R_{c} \\
\psi_{e}=B \frac{e^{-\beta\left(r_{1}+r_{2}\right)}}{r_{1} r_{2}}, \quad r_{1}, r_{2}>R_{c}
\end{gathered}
$$

La inclusión de un parámetro variacional extra permite una mayor flexibilidad en la función de prueba, lo que permite a su vez la obtención de energías más bajas que las de Marín y Cruz [29], como lo muestran los cálculos realizados en este trabajo y los que reporta DíazGarcía [71]. 
Rodríguez-Bautista et al. [69] resuelven las ecuaciones de Hartree-Fock para átomos confinados por paredes suaves.

Por otro lado, usando la función de prueba propuesta en este trabajo se obtienen valores más bajos para la energía, para la mayoría de radios de confinamiento y valores finitos de $V_{0}$. No sólo eso, sino que usando la función de prueba dada por (2.3) y (2.5), el cálculo variacional implementado en Mathematica 9 resulta más estable en comparación con las funciones propuestas por los otros autores, en el sentido de que el programa siempre encuentra el valor mínimo de la energía, en forma automatizada, como ocurrió en el caso del átomo de hidrógeno confinado (Sección 1.1.2).

En el caso de paredes impenetrables, la función de prueba utilizada por Díaz-García está dada por

$$
\psi=N\left(1-\frac{r_{1}}{R_{C}}\right)\left(1-\frac{r_{2}}{R_{c}}\right)\left(e^{-\alpha r_{1}} e^{-\beta r_{2}}+e^{-\beta r_{1}} e^{-\alpha r_{2}}\right)
$$

La cual fue propuesta originalmente por Gimarc [63], y es una función radialmente correlacionada. Debido a esto, para este caso de barreras impenetrables se obtienen energías más bajas con esta función que con la función empleada en este trabajo, además de que contiene dos parámetros variacionales en lugar de uno. 
Tabla 2.1. Parámetros variacionales óptimos $\alpha$ y $\gamma$, y energía del estado base $E$ del átomo de helio confinado como función del radio de confinamiento $R_{c}$ y la altura de la barrera $V_{0}$. Las energías están en hartrees y los radios en bohrs.

\begin{tabular}{|c|c|c|c|c|c|c|}
\hline$R_{c}$ & $\alpha$ & $\gamma$ & $E$ & Ref. [29] & Ref. [71] & Ref. [69] \\
\hline \multicolumn{7}{|c|}{$V_{0}=0$} \\
\hline 0.5 & 1.0382 & 0.4620 & -0.2384 & -0.2412 & -0.2450 & -0.6408 \\
\hline 0.9 & 1.8663 & 0.2602 & -1.9454 & & -1.9450 & \\
\hline 1.0 & 1.8934 & 0.1782 & -2.2172 & -2.0522 & -2.5536 & -2.3476 \\
\hline 1.5 & 1.6860 & 0.0051 & -2.7556 & -2.5086 & -2.7555 & -2.7298 \\
\hline 2.0 & 1.6654 & 0.0080 & -2.8277 & -2.6184 & -2.8277 & -2.8254 \\
\hline 3.0 & 1.6873 & 0.0109 & -2.8460 & -2.7579 & -2.8418 & -2.8588 \\
\hline 4.0 & 1.6876 & 0.0091 & -2.8475 & -2.8054 & -2.8472 & -2.8615 \\
\hline 6.0 & 1.6875 & 0.0100 & -2.8477 & -2.8341 & -2.8476 & -2.8617 \\
\hline \multicolumn{7}{|c|}{$V_{0}=5$} \\
\hline 0.5 & 1.7809 & 0.5056 & 4.6316 & 4.6722 & 4.6434 & 4.2233 \\
\hline 0.9 & 1.8032 & 0.4803 & -1.1346 & & -1.1272 & \\
\hline 1.0 & 1.7933 & 0.4616 & -1.6335 & -1.4621 & -1.6287 & -1.1871 \\
\hline 1.5 & 1.8178 & 0.2204 & -2.6254 & -2.4201 & -2.6253 & -2.3811 \\
\hline 2.0 & 1.7129 & 0.0051 & -2.8126 & -2.6179 & -2.8126 & -2.7116 \\
\hline 3.0 & 1.6876 & 0.0080 & -2.8460 & -2.7579 & -2.8459 & -2.8462 \\
\hline 4.0 & 1.6876 & 0.0050 & -2.8475 & -2.8054 & -2.8475 & -2.8602 \\
\hline 6.0 & 1.6875 & 0.0100 & -2.8477 & -2.8341 & -2.8476 & -2.8617 \\
\hline \multicolumn{7}{|c|}{$V_{0}=\infty$} \\
\hline 0.5 & 0.8428 & 1 & 22.9110 & 22.9229 & & \\
\hline 0.9 & 0.9250 & 1 & 2.6339 & 2.5117 & 2.5110 & \\
\hline 1.0 & 0.9476 & 1 & 1.1862 & 1.0626 & & \\
\hline 1.5 & 1.0700 & 1 & -1.7413 & -1.8456 & -1.8692 & \\
\hline 2.0 & 1.1984 & 1 & -2.4505 & -2.5285 & -2.5678 & \\
\hline 3.0 & 1.4043 & 1 & -2.7548 & -2.7935 & -2.8407 & \\
\hline 3.5 & 1.4703 & 1 & -2.7915 & -2.8189 & -2.8635 & \\
\hline 5.0 & 1.5766 & 1 & -2.8274 & -2.8392 & -2.8755 & \\
\hline 6.0 & 1.6100 & 1 & -2.8349 & -2.8426 & -2.8760 & \\
\hline
\end{tabular}




\subsection{Propiedades físicas}

\subsubsection{Polarizabilidad}

Para átomos con simetría esférica, la polarizabilidad en la aproximación de Kirkwood está dada por [64]

$$
\alpha_{d}=\frac{4}{9 N a_{0}}\left(\left\langle r_{1}^{2}\right\rangle+\left\langle r_{2}^{2}\right\rangle\right)^{2}
$$

Donde $N$ es el número de electrones y $a_{0}$ el radio de Bohr de la primera órbita del electrón.

En la Tabla 2.2 y en la Figura 2.1 se muestra la polarizabilidad del átomo de helio confinado, como función del radio de confinamiento. Para radios de confinamiento grandes, $\alpha_{d}$ tiende al valor $0.14618 \times 10^{-24} \mathrm{~cm}^{3}$. De manera similar al caso del átomo de hidrógeno confinado (Sección 1.1.2.1) para barreras penetrables, conforme el radio de confinamiento disminuye, la polarizabilidad del sistema disminuye hasta alcanzar un valor mínimo, y luego crece de forma monótona. Para barreras impenetrables, la polarizabilidad tiende a cero conforme $R_{c} \rightarrow 0$. Además, para un mismo radio de confinamiento, conforme la altura de la barrera aumenta (es decir, se vuelve menos penetrable), el valor de la polarizabilidad disminuye.

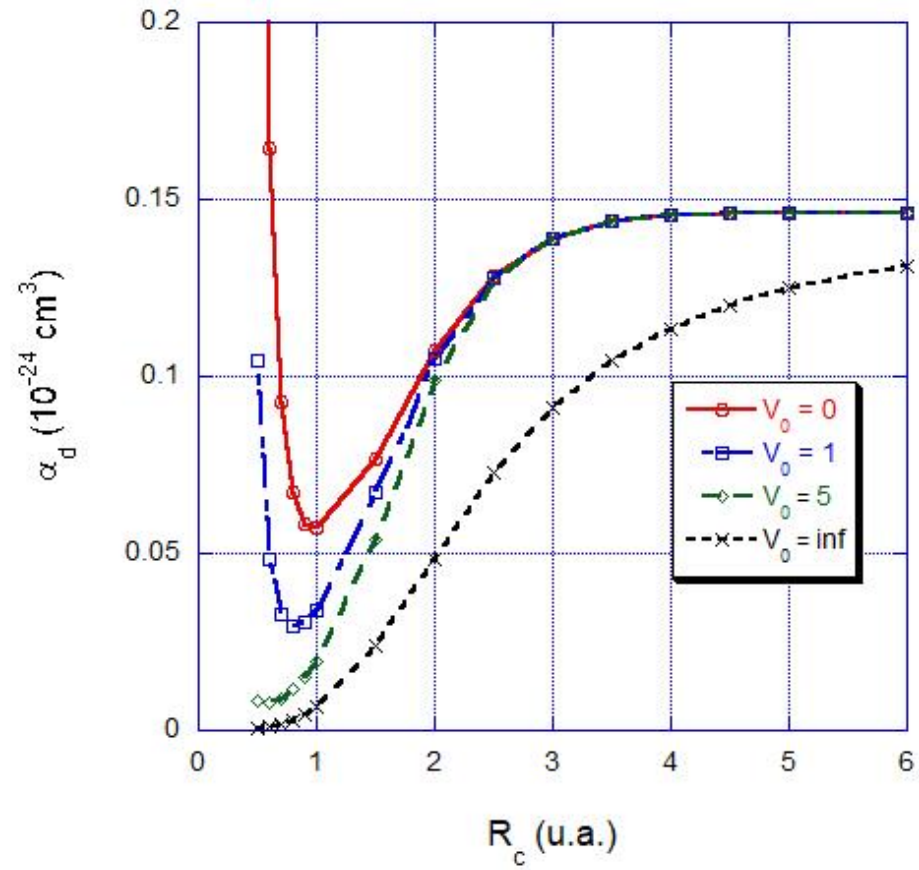

Figura 2.1. Polarizabilidad en la aproximación de Kirkwood (en unidades $10^{-24} \mathrm{~cm}^{3}$ ) como función de $R_{c}$ (bohrs) para $V_{0}=0,1,5, \infty$ hartrees. 
Tabla 2.2. Polarizabilidad en la aproximación de Kirkwood $\alpha_{d}$ (en unidades $10^{-24} \mathrm{~cm}^{3}$ ) como función de $R_{c}$ (bohrs) para $V_{0}=0,1,5, \infty$ hartrees.

\begin{tabular}{|l|l|l|l|}
\hline$R_{c}$ & \multicolumn{1}{|c|}{$\alpha_{d}$} & \multicolumn{1}{c|}{$R_{c}$} & \multicolumn{1}{|c|}{$\alpha_{d}$} \\
\hline \multicolumn{2}{|c|}{$V_{0}=0$} & \multicolumn{2}{|c|}{$V_{0}=1$} \\
\hline 0.8 & 0.06708 & 0.8 & 0.02953 \\
\hline 0.9 & 0.05844 & 0.9 & 0.03053 \\
\hline 1.0 & 0.05707 & 1.0 & 0.03392 \\
\hline 2.0 & 0.10715 & 2.0 & 0.10499 \\
\hline 2.5 & 0.12820 & 2.5 & 0.12798 \\
\hline 3.0 & 0.13917 & 3.0 & 0.13914 \\
\hline 3.5 & 0.14377 & 3.5 & 0.14373 \\
\hline 4.0 & 0.14543 & 4.0 & 0.14539 \\
\hline 5.0 & 0.14611 & 5.0 & 0.14613 \\
\hline 6.0 & 0.14618 & 6.0 & 0.14618 \\
\hline & $V_{0}=5$ & & $V_{0}=\infty$ \\
\hline 0.8 & 0.01145 & 0.8 & 0.00304 \\
\hline 0.9 & 0.01487 & 0.9 & 0.00460 \\
\hline 1.0 & 0.01921 & 1.0 & 0.00662 \\
\hline 1.5 & 0.05379 & 1.5 & 0.02368 \\
\hline 2.0 & 0.09890 & 2.0 & 0.04855 \\
\hline 2.5 & 0.12710 & 2.5 & 0.07266 \\
\hline 3.0 & 0.13908 & 3.0 & 0.09124 \\
\hline 3.5 & 0.14373 & 3.5 & 0.10437 \\
\hline 4.0 & 0.14544 & 4.0 & 0.11357 \\
\hline 5.0 & 0.14613 & 5.0 & 0.12493 \\
\hline 6.0 & 0.14618 & 6.0 & 0.13129 \\
\hline & & & \\
\hline
\end{tabular}




\subsubsection{Potenciales de ionización}

En la Tabla 2.3 se muestra la energía del estado base del ion $\mathrm{He}^{+}$confinado dentro de una cavidad esférica. Dado que se trata de un átomo hidrogenoide (un núcleo y un electrón), pueden hallarse los eigenvalores de la energía de forma exacta, usando la metodología que se presentó en la Sección 1.2, con la única diferencia de que el número atómico $Z=2$.

En la Figura 2.2 se grafican la energía del estado base del átomo de helio y del ion $\mathrm{He}^{+}$ confinados dentro de una cavidad esférica de altura $V_{0}=5$ hartrees. Al radio de confinamiento en el que se cruzan ambas gráficas se le conoce como "radio crítico de ionización". En este punto, $E(\mathrm{He})=E\left(\mathrm{He}^{+}\right)$, y uno de los electrones del helio se desliga del núcleo, convirtiéndose en una partícula libre dentro de la caja esférica, acompañado por un átomo ionizado $\mathrm{He}^{+}$. Una explicación más detallada, y las diferencias entre este "radio crítico de ionización" y el radio crítico en el cual el electrón escapa del confinamiento y pasa a un estado del continuo (en el caso de barreras penetrables), puede encontrarse en las referencias $[62,71]$.

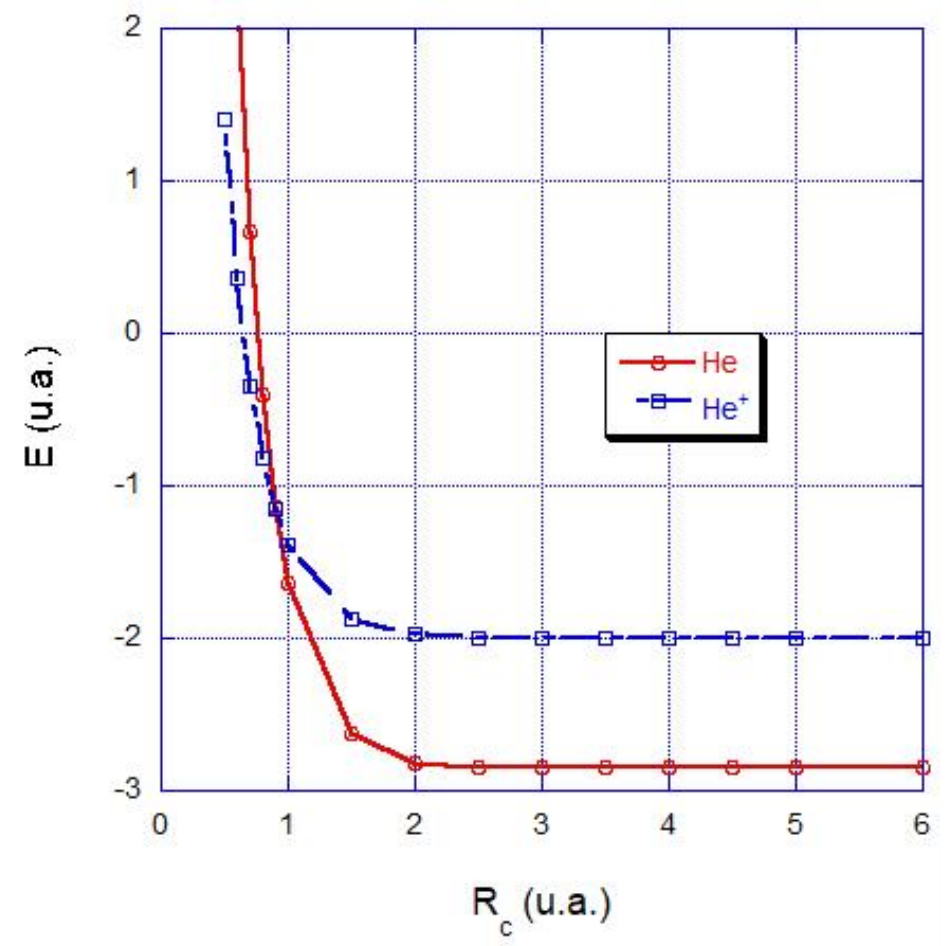

Figura 2.2. Energía del estado base del helio y del ion $\mathrm{He}^{+}$como función del radio de confinamiento, para una barrera de altura $V_{0}=5$ hartrees. 
Tabla 2.3. Energía del estado base del $\mathrm{He}^{+}$como función del radio de confinamiento $R_{c}$ y la altura de la barrera $V_{0}$. Las energías están en hartrees y las distancias en bohrs.

\begin{tabular}{|c|c|c|c|}
\hline$R_{C}$ & $E$ & $R_{C}$ & \multicolumn{2}{|c|}{$V_{0}=1$} \\
\hline \multicolumn{2}{|c|}{$V_{0}=0$} & \multicolumn{2}{|c|}{} \\
\hline 0.5 & -0.500000000000000 & 0.5 & 0.016467903750525 \\
\hline 0.9 & -1.613310924558766 & 0.9 & -1.472279083458153 \\
\hline 1.0 & -1.724875556967174 & 1.0 & -1.618989813021317 \\
\hline 1.5 & -1.948892331273591 & 1.5 & -1.922320005065366 \\
\hline 2.0 & -1.990698757603278 & 2.0 & -1.984252974299733 \\
\hline 2.5 & -1.998386685466300 & 2.5 & -1.996956478265043 \\
\hline 3.0 & -1.999732338173049 & 3.0 & -1.999441100587143 \\
\hline 3.5 & -1.999957069958803 & 3.5 & -1.999901616959221 \\
\hline 4.0 & -1.999993283418204 & 4.0 & -1.999983239394531 \\
\hline 4.5 & -1.999998968509123 & 4.5 & -1.999997216227079 \\
\hline 5.0 & -1.999999843845329 & 5.0 & -1.999999546860961 \\
\hline 6.0 & -1.999999996534142 & 6.0 & -1.999999988537036 \\
\hline & $V_{0}=5$ & & $V_{0}=\infty$ \\
\hline 0.5 & 1.396538016621507 & 0.5 & 9.495963464414655 \\
\hline 0.9 & -1.152700137671599 & 0.9 & 0.130225011167308 \\
\hline 1.0 & -1.385876376956845 & 1.0 & -0.500000000000000 \\
\hline 1.5 & -1.868980869540506 & 1.5 & -1.695869150933815 \\
\hline 2.0 & -1.971743522461750 & 2.0 & -1.933061208312088 \\
\hline 2.5 & -1.994195878833014 & 2.5 & -1.985668026365809 \\
\hline 3.0 & -1.998876772079087 & 3.0 & -1.997109145488460 \\
\hline 3.5 & -1.999793616153313 & 3.5 & -1.999450310205623 \\
\hline 4.0 & -1.999963594550954 & 4.0 & -1.999900401782962 \\
\hline 4.5 & -1.999993777902780 & 4.5 & -1.999982616876076 \\
\hline 5.0 & -1.999998962678796 & 5.0 & -1.999997053126102 \\
\hline 6.0 & -1.999999972751412 & 6.0 & -1.999999920637980 \\
\hline & & & \\
\hline & & & \\
\hline
\end{tabular}


De acuerdo a la Figura 2.2, el radio crítico de la primera ionización ocurre en $R_{I} \approx 0.894$ u.a., mientras que la segunda ionización, en la cual ambos electrones se encuentran desligados del núcleo $\left(E\left(H e^{+}\right)=0\right)$ ocurre en $R_{I I} \approx 0.642$ u.a. Sin embargo, como es señalado por Díaz-García y Cruz [62] este "método de superposición" no es adecuado para calcular radios críticos de ionización, ya que se están comparando sistemas confinados completamente distintos, que no están siendo gobernados por el mismo hamiltoniano. En su lugar, proponen que el cálculo de potenciales de ionización puede llevarse a cabo con una partición adecuada del hamiltoniano del átomo neutro. Para un átomo de dos electrones, proponen que el potencial de la primera ionización esté dado por

$$
\begin{gathered}
E_{I}=\left\langle\psi_{i}\left(r_{1}, r_{2}\right)\left|-\frac{\nabla_{2}^{2}}{2}-\frac{Z}{r_{2}}\right| \psi_{i}\left(r_{1}, r_{2}\right)\right\rangle+\left\langle\psi_{e}\left(r_{1}, r_{2}\right)\left|-\frac{\nabla_{2}^{2}}{2}+V_{0}\right| \psi_{e}\left(r_{1}, r_{2}\right)\right\rangle \\
+\left\langle\psi_{i}\left(r_{1}, r_{2}\right)\left|\frac{1}{\left|\vec{r}_{1}-\vec{r}_{2}\right|}\right| \psi_{i}\left(r_{1}, r_{2}\right)\right\rangle
\end{gathered}
$$

Donde $\psi_{i}\left(r_{1}, r_{2}\right)$ y $\psi_{e}\left(r_{1}, r_{2}\right)$ son las funciones de onda dentro y fuera de la cavidad, respectivamente, dadas por las ecuaciones (2.3) y (2.5).

En tanto que el potencial de la segunda ionización está dado por

$$
E_{I I}=\left\langle\psi_{i}\left(r_{1}, r_{2}\right)\left|-\frac{\nabla_{1}^{2}}{2}-\frac{Z}{r_{1}}\right| \psi_{i}\left(r_{1}, r_{2}\right)\right\rangle+\left\langle\psi_{e}\left(r_{1}, r_{2}\right)\left|-\frac{\nabla_{1}^{2}}{2}+V_{0}\right| \psi_{e}\left(r_{1}, r_{2}\right)\right\rangle
$$

De manera que la energía total del sistema se puede escribir como

$$
E=E_{I}+E_{I I}
$$

En la Tabla 2.4 se muestran los potenciales de ionización obtenidos por este método, para distintos radios de confinamiento y alturas de la barrera. Puede observarse que para radios de confinamiento grandes los potenciales tienden a los valores $E_{I}=0.8965$ hartrees y $E_{I I}=$ 1.9512 hartrees ( $24.4 \mathrm{eV}$ y $53.1 \mathrm{eV}$, respectivamente), los cuales son comparables con los valores obtenidos experimentalmente $\left(E_{I}=24.6 \mathrm{eV}\right.$ y $\left.E_{I I}=54.4 \mathrm{eV}\right)$ para el átomo de helio libre de confinamiento. También puede verse que conforme el sistema se encuentra más fuertemente confinado, la energía umbral necesaria para que el electrón quede desligado del núcleo es menor, hasta que $E_{I}$ y $E_{I I}$ alcanzan valores positivos, lo que implica que los electrones ya se encuentran desligados. 
Tabla 2.4. Primer potencial de ionización $E_{I}$, segundo potencial de ionización $E_{I I}$ y energía $E=$ $E_{I}+E_{I I}$ del estado base del átomo de helio confinado, como función del radio de confinamiento $R_{c}$ y la altura de la barrera $V_{0}$. Las energías están en hartrees y los radios en bohrs.

\begin{tabular}{|c|c|c|c|}
\hline$R_{C}$ & $E_{I}$ & $E_{I I}$ & $E$ \\
\hline \multicolumn{3}{|c|}{$V_{0}=0$} \\
\hline 0.5 & 0.1020 & -0.3405 & -0.2384 \\
\hline 1.0 & -0.4210 & -1.7962 & -2.2172 \\
\hline 1.5 & -0.7897 & -1.9658 & -2.7556 \\
\hline 2.0 & -0.8695 & -1.9582 & -2.8277 \\
\hline 2.5 & -0.8879 & -1.9540 & -2.8419 \\
\hline 3.0 & -0.8938 & -1.9522 & -2.8460 \\
\hline 3.5 & -0.8957 & -1.9515 & -2.8472 \\
\hline 4.0 & -0.8963 & -1.9513 & -2.8475 \\
\hline 4.5 & -0.8964 & -1.9512 & -2.8476 \\
\hline 5.0 & -0.8965 & -1.9512 & -2.8477 \\
\hline \multicolumn{5}{|c|}{$V_{0}=1$} \\
\hline 0.5 & 1.0878 & 0.1799 & 1.2677 \\
\hline 1.0 & -0.2610 & -1.7490 & -2.0100 \\
\hline 1.5 & -0.7525 & -1.9647 & -2.7172 \\
\hline 2.0 & -0.8646 & -1.9597 & -2.8243 \\
\hline 2.5 & -0.8875 & -1.9541 & -2.8417 \\
\hline 3.0 & -0.8938 & -1.9522 & -2.8460 \\
\hline 3.5 & -0.8957 & -1.9515 & -2.8472 \\
\hline 4.0 & -0.8962 & -1.9513 & -2.8475 \\
\hline 4.5 & -0.8964 & -1.9512 & -2.8476 \\
\hline 5.0 & -0.8965 & -1.9512 & -2.8477 \\
\hline \multicolumn{5}{|c|}{$V_{0}=5$} \\
\hline 0.5 & 3.3844 & 1.2472 & 4.6316 \\
\hline 1.0 & -0.0043 & -1.6292 & -1.6335 \\
\hline 1.5 & -0.6758 & -1.9496 & -2.6254 \\
\hline 2.0 & -0.8494 & -1.9632 & -2.8126 \\
\hline 2.5 & -0.8861 & -1.9547 & -2.8408 \\
\hline 3.0 & -0.8937 & -1.9523 & -2.8460 \\
\hline 3.5 & -0.8957 & -1.9515 & -2.8472 \\
\hline 4.0 & -0.8963 & -1.9513 & -2.8475 \\
\hline 4.5 & -0.8964 & -1.9512 & -2.8476 \\
\hline 5.0 & -0.8965 & -1.9512 & -2.8477 \\
\hline
\end{tabular}


Para hallar el radio crítico de ionización, deben analizarse las gráficas de los potenciales de ionización en función del radio de confinamiento, como las que se muestran en la Figura 2.3. El radio crítico correspondiente a la primera ionización $R_{c I}$ es aquel en el que $E_{I}=0$. De igual manera, el radio crítico correspondiente a la segunda ionización $R_{C I I}$ es el punto en el cual $E_{I I}=0$. Cabe recordar que estos radios críticos corresponden al punto en el cual cada electrón se desliga del núcleo, y son diferentes al radio de escape electrónico, el cual es el radio en el que el electrón escapa del confinamiento (en el caso de barreras penetrables) y pasa a un estado del continuo. En ese caso la energía del electrón debe ser igual a la altura de la barrera, $E=V_{0}$.

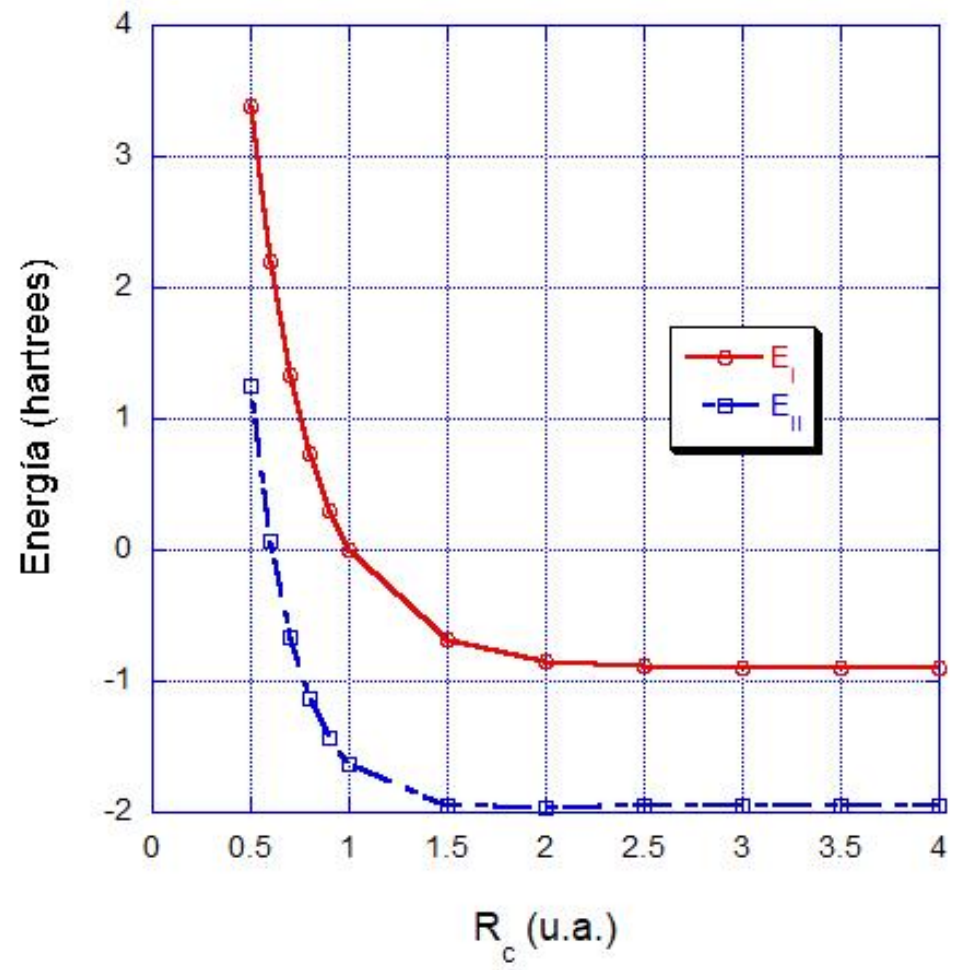

Figura 2.3. Potenciales de ionización del átomo de helio confinado como función del radio de confinamiento, para una barrera de altura $V_{0}=5$ hartrees. 
En la Tabla 2.5 se muestran los radios críticos para algunos valores finitos de la altura de la barrera. Puede apreciarse que entre más alta sea la barrera, es decir, entre más fuertemente confinado se encuentre el sistema, los radios críticos de ionización son cada vez mayores.

Tabla 2.5. Radios críticos de la primera y segunda ionización, $R_{C I}$ y $R_{C I I}$, respectivamente, del átomo de helio confinado, para diferentes alturas de la barrera $V_{0}$. Las energías están en hartrees y los radios en bohrs.

\begin{tabular}{|c|c|c|}
\hline$V_{0}$ & $R_{C I}$ & $R_{C I I}$ \\
\hline 0 & 0.733 & \\
\hline 1 & 0.875 & 0.525 \\
\hline 5 & 0.992 & 0.603 \\
\hline 10 & 1.089 & 0.642 \\
\hline
\end{tabular}

\subsubsection{Entropía informática de Shannon}

La entropía informática de Shannon en el espacio de configuraciones está dada por (ecuación (1.28)

$$
S_{r}=-\int d^{3} \vec{r} \rho(\vec{r}) \ln \rho(\vec{r})
$$

Donde $\rho(\vec{r})$ es la densidad electrónica normalizada a la unidad, que para un sistema atómico de dos electrones puede escribirse como [100]

$$
\rho(\vec{r})=\int d^{3} \vec{r}_{2}\left|\psi\left(\vec{r}, \vec{r}_{2}\right)\right|^{2}
$$

Para la región dentro de la esfera la densidad electrónica está dada por

$$
\rho_{i}(\vec{r})=\frac{1}{4 \pi}\left|\phi_{i}(r)\right|^{2} \int_{0}^{R_{c}}\left|\phi_{i}\left(r_{2}\right)\right|^{2} r_{2}^{2} d r_{2}
$$

Donde $\phi_{i}(r)$ para el átomo de helio confinado está dada por (2.4). En tanto que para la región externa se tiene 


$$
\rho_{e}(\vec{r})=\frac{1}{4 \pi}\left|\phi_{e}(r)\right|^{2} \int_{R_{c}}^{\infty}\left|\phi_{e}\left(r_{2}\right)\right|^{2} r_{2}^{2} d r_{2}
$$

La función $\phi_{e}(r)$ está dada por (2.6). De esta forma, la entropía informática de Shannon para el átomo de helio confinado dentro de una cavidad esférica penetrable queda como

$$
S_{r}=-\int_{r=0}^{r=R_{c}} d^{3} \vec{r} \rho_{i}(\vec{r}) \ln \rho_{i}(\vec{r})-\int_{r=R_{c}}^{r=\infty} d^{3} \vec{r} \rho_{e}(\vec{r}) \ln \rho_{e}(\vec{r})
$$

Desarrollando la ecuación (2.33), la entropía de Shannon finalmente se calcula como

$$
\begin{array}{rl}
S_{r}=-2 I_{1} \int_{0}^{R_{c}} & d r r^{2}\left|\phi_{i}(r)\right|^{2} \ln \left|\phi_{i}(r)\right|-2 I_{2} \int_{R_{c}}^{\infty} d r r^{2}\left|\phi_{e}(r)\right|^{2} \ln \left|\phi_{e}(r)\right| \\
& -2 \ln \frac{1}{4 \pi}-I_{1}^{2} \ln I_{1}-I_{2}^{2} \ln I_{2}
\end{array}
$$

Donde $I_{1}$ e $I_{2}$ son integrales auxiliares dadas por

$$
\begin{aligned}
& I_{1}=\int_{0}^{R_{C}} d r r^{2}\left|\phi_{i}(r)\right|^{2} \\
& I_{2}=\int_{0}^{\infty} d r r^{2}\left|\phi_{e}(r)\right|^{2}
\end{aligned}
$$

Juntas, estas integrales cumplen la condición de normalización de la función de onda

$$
I_{1}^{2}+I_{2}^{2}=1
$$

Para efectos de comparación, se calcula también la entropía de Shannon del ion $\mathrm{He}^{+}$ confinado dentro de una esfera penetrable. Por tratarse de un átomo hidrogenoide, con número atómico $Z=2$, pueden utilizarse las funciones de onda exactas del sistema, como se obtuvieron en la Sección 1.2.2, en tanto que la entropía de Shannon se calcula como se describe en la Sección 1.2.2.1.

En la Tabla 2.6 se muestran algunos valores obtenidos para la entropía de Shannon del átomo de helio y el ion $\mathrm{He}^{+}$confinados, como función del confinamiento. Para radios de confinamiento grandes, la entropía de Shannon toma el valor $S_{r}=2.5750$, para el helio, y $S_{r}=2.0653$, para el $\mathrm{He}^{+}$. Como en el caso del átomo de hidrógeno confinado, para barreras penetrables, conforme el radio de confinamiento disminuye, la entropía de Shannon disminuye (lo que indica que la densidad electrónica se encuentra cada vez más concentrada alrededor del núcleo) hasta alcanzar un valor mínimo, y finalmente crece de forma ilimitada 
(la probabilidad de hallar a los electrones fuera de la cavidad aumenta, es decir, el sistema está menos localizado). En el caso de barreras impenetrables, la entropía de Shannon disminuye de manera monótona conforme el radio de confinamiento disminuye. En la Figura 2.4 se muestra el comportamiento de la entropía informática de Shannon del átomo de helio como función del radio de confinamiento, para barreras de diferente altura.

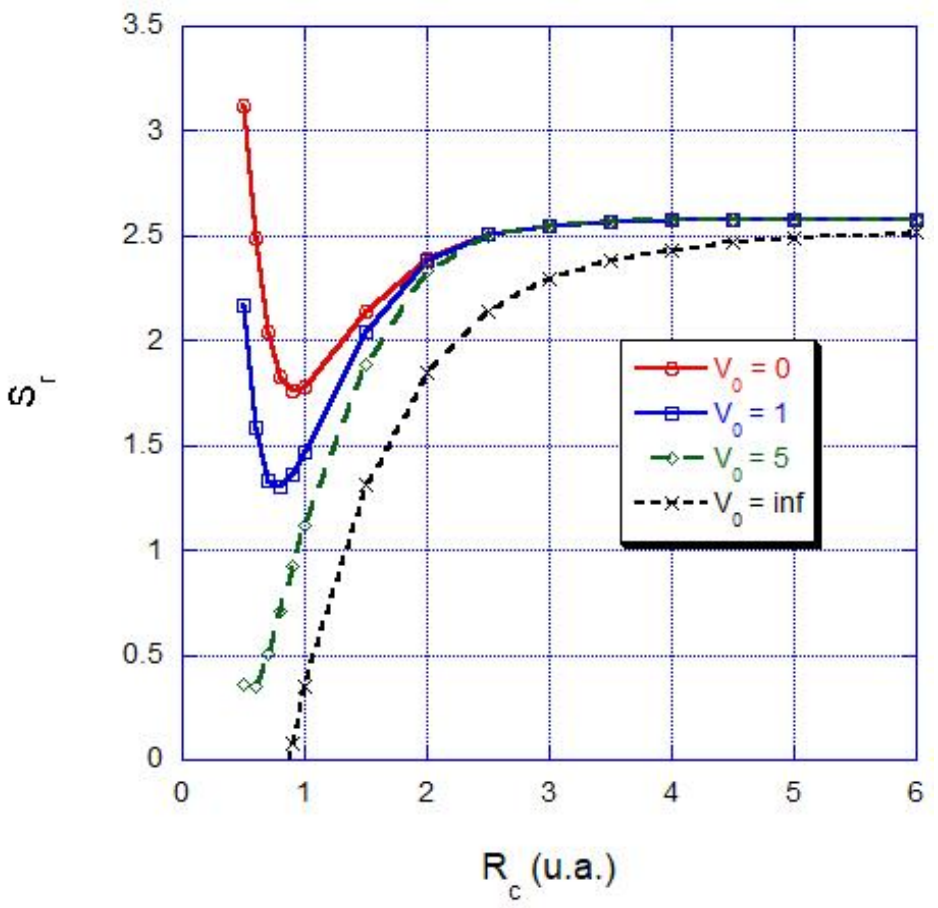

Figura 2.4. Entropía informática de Shannon del átomo de helio confinado, como función del radio de confinamiento, para diferentes valores de $V_{0}$. Las energías están en hartrees y las distancias en bohrs. 
Tabla 2.6. Entropía informática de Shannon del átomo de helio $\left(S_{r}^{(H e)}\right)$, y el ion $\mathrm{He}^{+}\left(\mathrm{S}_{r}^{\left(H e^{+}\right)}\right)$, confinados dentro de una barrera esférica de radio $R_{c}$ y altura $V_{0}$. Las energías están en hartrees y los radios en bohrs.

\begin{tabular}{|c|c|c|c|c|c|}
\hline$R_{c}$ & $S_{r}^{(\mathrm{He})}$ & $S_{r}^{(\mathrm{He})}$ & $R_{c}$ & $S_{r}^{(\mathrm{He})}$ & $S_{r}^{(\mathrm{He})}$ \\
\hline \multicolumn{3}{|c|}{$V_{0}=0$} & \multicolumn{3}{|c|}{$V_{0}=1$} \\
\hline 0.5 & 3.1167 & 1.8593 & 0.5 & 2.1668 & 1.2177 \\
\hline 0.9 & 1.7602 & 1.4411 & 0.9 & 1.3649 & 1.2057 \\
\hline 1.0 & 1.7799 & 1.5138 & 1.0 & 1.4717 & 1.3093 \\
\hline 1.5 & 2.1408 & 1.8323 & 1.5 & 2.0458 & 1.7281 \\
\hline 2.0 & 2.3956 & 1.9884 & 2.0 & 2.3798 & 1.9422 \\
\hline 2.5 & 2.5071 & 2.0441 & 2.5 & 2.5058 & 2.0274 \\
\hline 3.0 & 2.5520 & 2.0602 & 3.0 & 2.5518 & 2.0551 \\
\hline 3.5 & 2.5679 & 2.0642 & 3.5 & 2.5677 & 2.0628 \\
\hline 4.0 & 2.5729 & 2.0651 & 4.0 & 2.5728 & 2.0647 \\
\hline 4.5 & 2.5745 & 2.0652 & 4.5 & 2.5745 & 2.0652 \\
\hline 5.0 & 2.5748 & 2.0653 & 5.0 & 2.5748 & 2.0653 \\
\hline 6.0 & 2.5750 & 2.0653 & 6.0 & 2.5750 & 2.0653 \\
\hline \multicolumn{3}{|c|}{$V_{0}=5$} & & & $V_{0}=\infty$ \\
\hline 0.5 & 0.3573 & 0.1914 & 0.5 & -1.5382 & -1.5504 \\
\hline 0.9 & 0.9215 & 0.8117 & 0.9 & 0.0813 & 0.0498 \\
\hline 1.0 & 1.1202 & 0.9717 & 1.0 & 0.3550 & 0.3172 \\
\hline 1.5 & 1.8899 & 1.5635 & 1.5 & 1.3158 & 1.2369 \\
\hline 2.0 & 2.3332 & 1.8690 & 2.0 & 1.8493 & 1.7148 \\
\hline 2.5 & 2.5004 & 2.0001 & 2.5 & 2.1389 & 1.9380 \\
\hline 3.0 & 2.5515 & 2.0466 & 3.0 & 2.2946 & 2.0257 \\
\hline 3.5 & 2.5677 & 2.0605 & 3.5 & 2.3817 & 2.0545 \\
\hline 4.0 & 2.5730 & 2.0642 & 4.0 & 2.4338 & 2.0626 \\
\hline 4.5 & 2.5743 & 2.0650 & 4.5 & 2.4670 & 2.0647 \\
\hline 5.0 & 2.5748 & 2.0652 & 5.0 & 2.4897 & 2.0652 \\
\hline 6.0 & 2.5750 & 2.0653 & 6.0 & 2.5175 & 2.0653 \\
\hline
\end{tabular}


En la Figura 2.5 se observa la evolución de la entropía de Shannon del átomo de helio y el ion $\mathrm{He}^{+}$confinados dentro de una cavidad esférica, como función de la compresión. Puede observarse que la entropía de Shannon del $\mathrm{He}^{+}$se encuentra por debajo de la entropía del helio, lo que sugiere que la densidad electrónica del primero se encuentra más localizada alrededor del núcleo que la del segundo. Esto se puede explicar posiblemente debido a que el helio tiene dos electrones y el $\mathrm{He}^{+}$sólo uno, lo que hace que la nube electrónica del helio sea más grande y por ende esté menos localizada.

El decaimiento más rápido de la curva de la entropía de Shannon del helio, en comparación a la del $\mathrm{He}^{+}$, sugiere que el helio, debido al mayor tamaño de su nube electrónica, sufre una compresión más rápida que la de su contraparte ionizada.

A medida que el radio de confinamiento disminuye y la altura de la barrera aumenta, la entropía de Shannon del helio tiende asintóticamente a la del $\mathrm{He}^{+}$. Este comportamiento se puede explicar observando los radios críticos de ionización, obtenidos en la Sección 2.2.2. Para radios de confinamiento menores a este radio crítico, el primer electrón del helio se encuentra desligado del núcleo, por lo que el sistema se comporta similar al $\mathrm{He}^{+}$confinado, junto con un electrón que se mueve libremente dentro o fuera de la cavidad. Aun así, existen algunas diferencias pequeñas entre ambos sistemas. Por ejemplo, para $V_{0}=5$ hartrees y un radio de aproximadamente 0.5 bohrs, la entropía del átomo de helio confinado tiene un mínimo, posiblemente debido a la probabilidad de que el electrón desligado pueda escapar del confinamiento. Este comportamiento sugiere que, aunque débilmente, el electrón desligado continúa interactuando con el resto del sistema. 

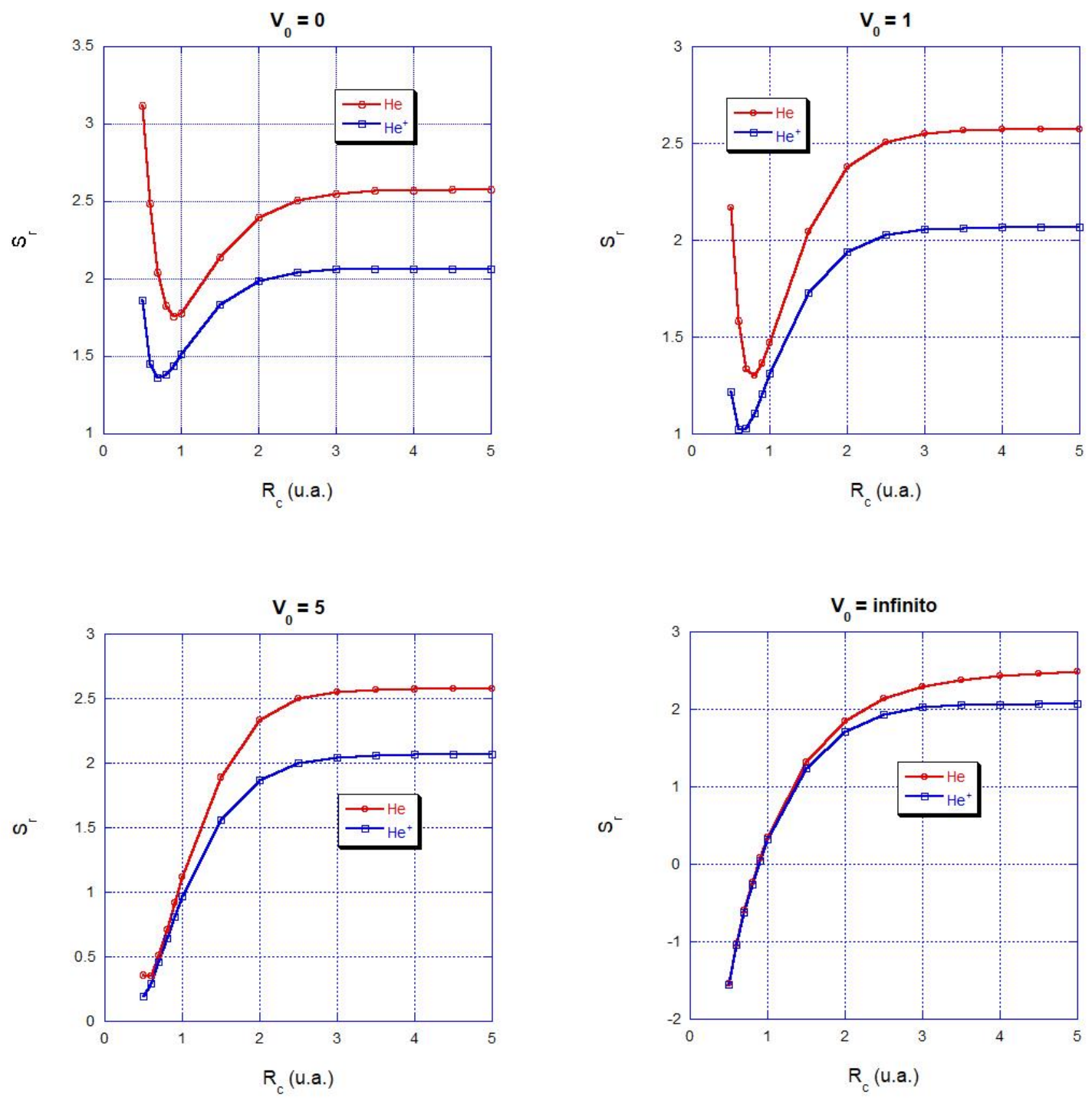

Figura 2.5. Entropía informática de Shannon del átomo de helio y el ion $\mathrm{He}^{+}$confinados, como función del radio de confinamiento, para diferentes valores de $V_{0}$. Las energías están en hartrees y las distancias en bohrs.

\subsubsection{Núcleo de tamaño finito}

Para hallar el efecto que tiene considerar un núcleo de tamaño finito en las energías del átomo de helio confinado, se recurrirá al uso de teoría de perturbaciones a primer orden, como en el caso del AHC, discutido en la sección 1.2.2.1. 
En unidades atómicas, el hamiltoniano de un átomo helioide, considerando un núcleo de tamaño finito con una distribución de carga esférica y uniforme de magnitud $+Z e$, confinado dentro de una cavidad esférica, está dado por

$$
H=\frac{p_{1}^{2}}{2}+\frac{p_{2}^{2}}{2}+V\left(r_{1}, r_{2}\right)+V_{c}
$$

En esta ecuación se está considerando al núcleo fijo en el centro de la esfera. El potencial $V\left(r_{1}, r_{2}\right)$ está dado por

$$
V\left(r_{1}, r_{2}\right)=\left\{\begin{array}{rr}
\frac{Z}{2 r_{0}}\left[\left(\frac{r_{1}}{r_{0}}\right)^{2}+\left(\frac{r_{2}}{r_{0}}\right)^{2}-6\right]+\frac{1}{r_{12}}, & r_{1}, r_{2}<r_{0} \\
-\frac{Z}{r_{1}}-\frac{Z}{r_{2}}+\frac{1}{r_{12}}, & r_{1}, r_{2} \geq r_{0}
\end{array}\right.
$$

$r_{0}$ es el radio del núcleo. $r_{1}$ y $r_{2}$ son las distancias radiales de cada electrón al centro del núcleo y $r_{12}$ es la distancia entre ambos electrones.

El potencial de confinamiento $V_{c}$ está dado por

$$
V_{c}=\left\{\begin{aligned}
0, & r_{1}, r_{2}<R_{c} \\
V_{0}, & r_{1}, r_{2} \geq R_{c}
\end{aligned}\right.
$$

Sumando y restando la cantidad $Z / r_{1}+Z / r_{2}-1 / r_{12}$, el hamiltoniano (2.38) puede reescribirse como

$$
H=\frac{p_{1}^{2}}{2}+\frac{p_{2}^{2}}{2}-\frac{Z}{r_{1}}-\frac{Z}{r_{2}}+\frac{1}{r_{12}}+V_{c}+V\left(r_{1}, r_{2}\right)+\frac{Z}{r_{1}}+\frac{Z}{r_{2}}-\frac{1}{r_{12}}
$$

Que se puede escribir como

$$
H=H^{(0)}+H^{\prime}
$$

Donde $H^{(0)}$ es el hamiltoniano de un átomo helioide confinado dentro de una barrera esférica, considerando su núcleo como una partícula puntual (ecuación (2.1)), el cual se tomará como el sistema no perturbado. En tanto que

$$
H^{\prime}=V\left(r_{1}, r_{2}\right)+\frac{Z}{r_{1}}+\frac{Z}{r_{2}}-\frac{1}{r_{12}}
$$

El cual se puede escribir, después de un poco de álgebra, en la siguiente forma 


$$
H^{\prime}=\left\{\begin{array}{rr}
\frac{Z}{2 r_{0}}\left[\left(\frac{r_{1}}{r_{0}}\right)^{2}+\left(\frac{r_{2}}{r_{0}}\right)^{2}-6\right]+\frac{Z}{r_{1}}+\frac{Z}{r_{2}}, & r_{1}, r_{2}<r_{0} \\
0, & r_{1}, r_{2} \geq r_{0}
\end{array}\right.
$$

Este término se tomará como una pequeña perturbación.

Las funciones de onda espaciales del sistema no perturbado, para el estado singulete, son

$$
\psi^{(0)}\left(\vec{r}_{1}, \vec{r}_{2}\right)=\phi^{(0)}\left(\vec{r}_{1}\right) \phi^{(0)}\left(\vec{r}_{2}\right)
$$

Donde

$$
\phi^{(0)}\left(\vec{r}_{i}\right)=R^{(0)}\left(r_{i}\right) Y_{l m}\left(\theta_{i}, \varphi_{i}\right), \quad i=1,2
$$

$R^{(0)}\left(r_{i}\right)$ es la función de onda radial del i-ésimo electrón. Dado que la perturbación se encuentra en la región $r_{1}, r_{2}<r_{0}<R_{c}, R^{(0)}\left(r_{i}\right)$ tiene la forma de la ecuación (2.4). En tanto que $Y_{l m}\left(\theta_{i}, \varphi_{i}\right)$ son los armónicos esféricos.

Usando teoría de perturbaciones independientes del tiempo, la corrección a primer orden está dada por el valor esperado de la perturbación, en los estados no perturbados

$$
E^{(1)}=\left\langle\psi^{(0)}\left(\vec{r}_{1}, \vec{r}_{2}\right)\left|H^{\prime}\right| \psi^{(0)}\left(\vec{r}_{1}, \vec{r}_{2}\right)\right\rangle
$$

$H^{\prime}$ se puede escribir como

$$
H^{\prime}=\left\{\begin{aligned}
W\left(r_{1}\right)+W\left(r_{2}\right), & r_{1}, r_{2}<r_{0} \\
0, & r_{1}, r_{2} \geq r_{0}
\end{aligned}\right.
$$

Donde

$$
W\left(r_{i}\right)=\frac{Z}{2 r_{0}}\left[\left(\frac{r_{i}}{r_{0}}\right)^{2}-3\right]+\frac{Z}{r_{i}}, \quad i=1,2
$$

De esta forma, considerando que las funciones $\phi^{(0)}\left(\vec{r}_{i}\right)$ están normalizadas

$$
E^{(1)}=\left\langle\phi^{(0)}\left(\vec{r}_{1}\right)\left|W\left(r_{1}\right)\right| \phi^{(0)}\left(\vec{r}_{1}\right)\right\rangle+\left\langle\phi^{(0)}\left(\vec{r}_{2}\right)\left|W\left(r_{2}\right)\right| \phi^{(0)}\left(\vec{r}_{2}\right)\right\rangle
$$

Donde

$$
\left\langle\phi^{(0)}(\vec{r})|W(r)| \phi^{(0)}(\vec{r})\right\rangle=\frac{Z}{2 r_{0}} \int_{0}^{r_{0}}\left|R^{(0)}(r)\right|^{2}\left[\left(\frac{r}{r_{0}}\right)^{2}-3+\frac{2 r_{0}}{r}\right] r^{2} d r
$$

Para $Z$ pequeña se puede considerar $r_{0} \ll a_{0}$. En ese caso, dado que la extensión espacial de la función de onda $R^{(0)}\left(r_{i}\right)$ es mucho mayor que la región $r_{i} \leq r_{0}$ en la cual $H^{\prime}$ es distinta de cero, se puede hacer la aproximación 


$$
R^{(0)}\left(r_{i}\right) \approx R^{(0)}(0), \quad r_{i}<r_{0}
$$

En cuyo caso, la ecuación (2.51) se reduce a

$$
E^{(1)}=\frac{2 Z}{10} r_{0}^{2}\left|R^{(0)}(0)\right|^{2}
$$

Para el estado base del átomo de helio confinado, la función de onda radial en la región $r_{1}, r_{2} \leq R_{c}$ es la misma que se ha empleado a lo largo de este capítulo (ecuación (2.4))

$$
R^{(0)}\left(r_{i}\right)=A j_{0}\left(\frac{\pi r_{i}}{R_{c}}\right) e^{-\alpha r_{i}}
$$

Donde $A$ es una constante de normalización. Sustituyendo (2.54) en (2.53), la corrección a la energía del estado base del átomo de helio confinado debida al núcleo de tamaño finito queda como

$$
E^{(1)}=\frac{Z}{5} r_{0}^{2}|A|^{2}
$$

Tomemos como ejemplo un átomo de helio $(Z=2)$, con un núcleo de ${ }^{4} \mathrm{He}$ al cual le corresponde un radio nuclear de $r_{0}=1.5 \mathrm{fm}[82]$.

En la Tabla 2.7 y en la Figura 2.6 se muestra el comportamiento de la corrección a la energía del estado base del átomo de helio confinado debida al efecto del núcleo de tamaño finito, como función del radio de confinamiento y la altura de la barrera. Como puede observarse, esta corrección resulta ser muy pequeña (del orden de $10^{-10}$ hartrees) por lo que es válido que se considere como una pequeña perturbación. Para radios de confinamiento grandes, la corrección de núcleo finito tiende al valor $E^{(1)}=61.7783 \times 10^{-10}$ hartrees. Conforme aumenta el confinamiento del sistema, también lo hace $E^{(1)}$, ya que los electrones se encuentran más cerca del núcleo. En el caso de barreras penetrables, la corrección de núcleo finito alcanza un valor máximo, y ya que para sistemas fuertemente confinados la probabilidad de hallar al electrón fuera de la barrera (y por tanto más lejos del núcleo) es alta, $E^{(1)}$ decae rápidamente. Esto no ocurre en el caso del confinamiento dentro de barreras impenetrables: ya que no hay manera de que los electrones escapen del confinamiento, conforme el radio de la barrera disminuye, los electrones tienden a encontrarse más próximos al núcleo, por lo que su interacción con éste es cada vez mayor y por tanto la corrección de núcleo finito aumenta de forma ilimitada. Además, para un radio de confinamiento fijo, 
puede observarse que a medida que la altura de la barrera aumenta (es decir, para barreras menos penetrables), la corrección de núcleo finito también aumenta.

Comparando los valores de la Tabla 2.7 con los de las Tablas 1.18 a 1.22, puede apreciarse que la corrección de núcleo finito es mayor para el helio que para el hidrógeno.

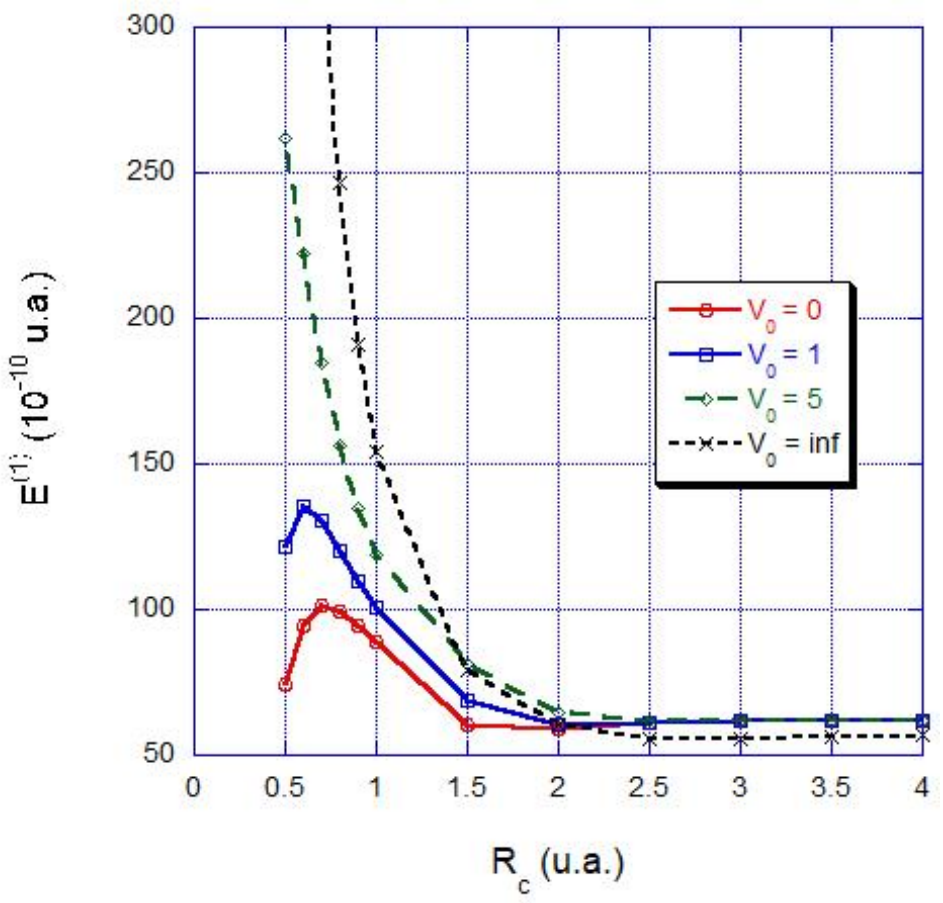

Figura 2.6. Corrección de núcleo finito $E^{(1)}$ a la energía del estado base del átomo de helio confinado, como función del radio de confinamiento $R_{c}$ y la altura de la barrera $V_{0}$. Las energías están en hartrees y los radios en bohrs. 
Tabla 2.7. Corrección de núcleo de tamaño finito $E^{(1)}$ a la energía del estado base del átomo de helio confinado dentro de una esfera penetrable, como función del radio de confinamiento $R_{c}$ y la altura de la barrera $V_{0}$. Las energías están en hartrees y las distancias en bohrs.

\begin{tabular}{|c|c|c|c|}
\hline$R_{C}$ & $E^{(1)}\left(10^{-10}\right)$ & $R_{c}$ & $E^{(1)}\left(10^{-10}\right)$ \\
\hline \multicolumn{2}{|c|}{$V_{0}=0$} & \multicolumn{2}{|c|}{$V_{0}=1$} \\
\hline 0.5 & 74.1197 & 0.5 & 121.8017 \\
\hline 0.9 & 94.5557 & 0.9 & 109.5097 \\
\hline 1.0 & 89.2539 & 1.0 & 100.4024 \\
\hline 1.5 & 60.7245 & 1.5 & 68.5980 \\
\hline 2.0 & 59.2447 & 2.0 & 60.5429 \\
\hline 2.5 & 61.2990 & 2.5 & 61.3968 \\
\hline 3.0 & 61.7548 & 3.0 & 61.7653 \\
\hline 3.5 & 61.8008 & 3.5 & 61.8125 \\
\hline 4.0 & 61.7897 & 4.0 & 61.8008 \\
\hline 4.5 & 61.7789 & 4.5 & 61.7785 \\
\hline 5.0 & 61.7804 & 5.0 & 61.7784 \\
\hline 6.0 & 61.7783 & 6.0 & 61.7783 \\
\hline & $V_{0}=5$ & & $V_{0}=\infty$ \\
\hline 0.5 & 262.1822 & 0.5 & 764.6064 \\
\hline 0.9 & 134.6519 & 0.9 & 191.2544 \\
\hline 1.0 & 118.6245 & 1.0 & 154.3727 \\
\hline 1.5 & 81.5438 & 1.5 & 79.2882 \\
\hline 2.0 & 64.4865 & 2.0 & 60.6423 \\
\hline 2.5 & 61.7808 & 2.5 & 55.9034 \\
\hline 3.0 & 61.7865 & 3.0 & 55.4124 \\
\hline 3.5 & 61.8125 & 3.5 & 56.0927 \\
\hline 4.0 & 61.7889 & 4.0 & 56.9591 \\
\hline 4.5 & 61.7897 & 4.5 & 57.7440 \\
\hline 5.0 & 61.7784 & 5.0 & 58.3825 \\
\hline 6.0 & 61.7783 & 6.0 & 59.3254 \\
\hline
\end{tabular}




\subsection{Conclusiones}

Se calculó la energía del estado base del átomo de helio confinado dentro de una cavidad esférica, como función del radio de confinamiento y la altura de la barrera. Para esto, se empleó el método variacional de Rayleigh-Ritz. En la función de prueba se incluyeron tres parámetros variacionales, lo cual condujo a la obtención de energías más bajas que las obtenidas por otros autores. No sólo eso, sino que la función de onda propuesta en este trabajo resulta ser más estable para la realización de cálculos numéricos que las empleadas por DíazGarcía y Marín y Cruz. Los cálculos de la energía pueden mejorarse utilizando una función de prueba que incluya correlación electrónica. Además, en este modelo de átomo helioide confinado, se está considerando que ambos electrones siempre se encuentran juntos, ya sea dentro de la cavidad o fuera de ésta. Un modelo más apropiado debería incluir la posibilidad de hallar a uno de los electrones dentro de la cavidad, y al otro fuera.

Se estudiaron algunas propiedades físicas del átomo de helio confinado, poco analizadas en la literatura. Por ejemplo, en el caso de la polarizabilidad, se empleó la aproximación de Kirkwood para su obtención. Cualitativamente se encontró un comportamiento similar que el de la polarizabilidad en el átomo de hidrógeno confinado, sin embargo los valores de la polarizabilidad del helio resultan ser menores a los del hidrógeno bajo las mismas condiciones de confinamiento.

Se calcularon los potenciales de ionización del helio confinado, usando el método de partición del hamiltoniano, propuesto por Díaz-García y Cruz [62]. Se obtuvieron radios críticos de ionización similares a los de estos autores. Conforme la altura de la barrera aumenta, los radios críticos de ionización también son mayores. En otras palabras, una mayor compresión del sistema ioniza al átomo más fácilmente.

Parece haber cierta relación entre los potenciales de ionización y la entropía informática de Shannon en el espacio de configuraciones, ya que conforme el radio de confinamiento disminuye, la entropía de Shannon del helio tiende a la del ion $\mathrm{He}^{+}$, lo que sugiere que bajo los efectos de un confinamiento fuerte la densidad electrónica de ambos sistemas comienza a comportarse de manera similar; sin embargo, existen algunas diferencias, sobre todo para barreras penetrables, que sugieren que el electrón desligado continúa interactuando débilmente con el átomo ionizado. 
Se calculó la contribución a la energía del estado base del átomo de helio confinado al tomar en cuenta el tamaño finito del núcleo. Aunque esta contribución es mayor que en el caso del átomo de hidrógeno, estudiado en el capítulo anterior, y que conforme el radio de confinamiento disminuye el efecto del núcleo de tamaño finito aumenta considerablemente, se demostró que este corrimiento de la energía es del orden de $10^{-10}$ hartrees, por lo que el uso de teoría de perturbaciones sigue siendo válido. 


\section{Capítulo 3}

\section{Átomo de litio confinado}

En este capítulo se estudia la corrección de núcleo de tamaño finito a la energía de los estados

$1 s^{2} 2 s$ y $1 s^{2} 2 p_{z}$ del átomo de litio confinado dentro de una barrera esférica de paredes impenetrables, usando teoría de perturbaciones a primer orden, como en capítulos anteriores se calculó para los átomos de hidrógeno y helio confinados. Las energías del sistema no perturbado (núcleo como una partícula puntual) fueron obtenidas por F. A. Duarte [65, 111], usando el método variacional de Rayleigh-Ritz.

\subsection{Sistema no perturbado (núcleo puntual)}

\subsubsection{Estado $1 s^{2} 2 s$}

En esta sección daremos breve descripción del cálculo de las energías del estado $1 s^{2} 2 s$ realizado por Duarte [65]. En unidades atómicas, el hamiltoniano de un átomo de litio confinado dentro de una barrera esférica de paredes impenetrables de radio $R_{c}$, cuyo núcleo se encuentra anclado en su centro, está dado por

$$
H^{(0)}=-\frac{1}{2} \sum_{i=1}^{3} \nabla_{i}^{2}-Z \sum_{i=1}^{3} \frac{1}{r_{i}}+\sum_{i=1}^{3} \sum_{j>i}^{3} \frac{1}{r_{i j}}+V_{c}
$$

Donde el primer término de la derecha corresponde a la energía cinética de cada electrón, el segundo término es la interacción coulombiana entre cada electrón y el núcleo, el tercer término corresponde a la interacción (repulsión) entre cada par de electrones y $V_{c}$ es el potencial de confinamiento 


$$
V_{c}=\left\{\begin{array}{cc}
0, & r_{1}, r_{2}, r_{3} \leq R_{c} \\
\infty, & r_{1}, r_{2}, r_{3}>R_{c}
\end{array}\right.
$$

Se propone una función de prueba en forma de un determinante de Slater construido mediante funciones de espín-orbitales hidrogenoides $1 s$ y $2 s$

$$
\Psi^{(0)}\left(\vec{r}_{1}, \vec{r}_{2}, \vec{r}_{3}\right)=\frac{1}{\sqrt{6}}\left|\begin{array}{lll}
\phi_{1}\left(\vec{r}_{1}\right) \alpha\left(\vec{r}_{1}\right) & \phi_{2}\left(\vec{r}_{1}\right) \beta\left(\vec{r}_{1}\right) & \phi_{3}\left(\vec{r}_{1}\right) \alpha\left(\vec{r}_{3}\right) \\
\phi_{1}\left(\vec{r}_{2}\right) \alpha\left(\vec{r}_{2}\right) & \phi_{2}\left(\vec{r}_{2}\right) \beta\left(\vec{r}_{2}\right) & \phi_{3}\left(\vec{r}_{2}\right) \alpha\left(\vec{r}_{2}\right) \\
\phi_{1}\left(\vec{r}_{3}\right) \alpha\left(\vec{r}_{3}\right) & \phi_{2}\left(\vec{r}_{3}\right) \beta\left(\vec{r}_{3}\right) & \phi_{3}\left(\vec{r}_{3}\right) \alpha\left(\vec{r}_{3}\right)
\end{array}\right|
$$

Donde las funciones $\alpha(\vec{r})$ y $\beta(\vec{r})$ representan las funciones de espín. Desarrollando el determinante, la función $\Psi^{(0)}\left(\vec{r}_{1}, \vec{r}_{2}, \vec{r}_{3}\right)$ se puede escribir como

$$
\begin{aligned}
\Psi^{(0)}\left(\vec{r}_{1}, \vec{r}_{2}, \vec{r}_{3}\right) & =\Psi_{1} \beta\left(\vec{r}_{1}\right) \alpha\left(\vec{r}_{2}\right) \alpha\left(\vec{r}_{3}\right)+\Psi_{2} \alpha\left(\vec{r}_{1}\right) \beta\left(\vec{r}_{2}\right) \alpha\left(\vec{r}_{3}\right) \\
& +\Psi_{3} \alpha\left(\vec{r}_{1}\right) \alpha\left(\vec{r}_{2}\right) \beta\left(\vec{r}_{3}\right)
\end{aligned}
$$

Donde la parte espacial queda definida por las funciones

$$
\begin{aligned}
& \Psi_{1}=\frac{1}{\sqrt{6}}\left[\phi_{2}\left(\vec{r}_{1}\right) \phi_{3}\left(\vec{r}_{2}\right) \phi_{1}\left(\vec{r}_{3}\right)-\phi_{2}\left(\vec{r}_{1}\right) \phi_{1}\left(\vec{r}_{2}\right) \phi_{3}\left(\vec{r}_{3}\right)\right] \\
& \Psi_{2}=\frac{1}{\sqrt{6}}\left[\phi_{1}\left(\vec{r}_{1}\right) \phi_{2}\left(\vec{r}_{2}\right) \phi_{3}\left(\vec{r}_{3}\right)-\phi_{3}\left(\vec{r}_{1}\right) \phi_{2}\left(\vec{r}_{2}\right) \phi_{1}\left(\vec{r}_{3}\right)\right] \\
& \Psi_{3}=\frac{1}{\sqrt{6}}\left[\phi_{3}\left(\vec{r}_{1}\right) \phi_{1}\left(\vec{r}_{2}\right) \phi_{2}\left(\vec{r}_{3}\right)-\phi_{1}\left(\vec{r}_{1}\right) \phi_{3}\left(\vec{r}_{2}\right) \phi_{2}\left(\vec{r}_{3}\right)\right]
\end{aligned}
$$

Cada función hidrogenoide está dada por

$$
\begin{gathered}
\phi_{1}(\vec{r})=N_{1} e^{-\alpha r}\left(1-\frac{r}{R_{c}}\right) Y_{00}(\theta, \varphi) \\
\phi_{2}(\vec{r})=N_{2} e^{-\beta r}\left(1-\frac{r}{R_{c}}\right) Y_{00}(\theta, \varphi) \\
\phi_{3}(\vec{r})=N_{3}(2-\delta r) e^{-\gamma r / 2}\left(1-\frac{r}{R_{c}}\right) Y_{00}(\theta, \varphi)
\end{gathered}
$$

$N_{1}, N_{2}$ y $N_{3}$ son constantes de normalización, $\alpha, \beta, \gamma$ y $\delta$ son parámetros variacionales y $Y_{00}(\theta, \varphi)$ son los armónicos esféricos.

Para asegurar que la función de onda se comporte adecuadamente en la frontera, se introduce la función de corte $f_{\text {cut }}=1-r / R_{c}$ a cada orbital hidrogenoide, de tal manera que la función de onda se anule en $r=R_{c}$.

Haciendo uso del método variacional directo se procede a calcular la energía del sistema 


$$
E^{(0)}(\alpha, \beta, \gamma, \delta)=\frac{\int \Psi^{(0) *} H^{(0)} \Psi^{(0)} d^{3} \vec{r}}{\int \Psi^{(0) *} \Psi^{(0)} d^{3} \vec{r}}
$$

El valor esperado del hamiltoniano está dado por

$$
\begin{aligned}
\left\langle\Psi^{(0)}\left|H^{(0)}\right| \Psi^{(0)}\right\rangle & =\int \Psi^{(0) *} H^{(0)} \Psi^{(0)} d^{3} \vec{r} \\
& =\int \Psi^{(0) *}\left(-\frac{1}{2} \sum_{i=1}^{3} \nabla_{i}^{2}-Z \sum_{i=1}^{3} \frac{1}{r_{i}}+\sum_{i=1}^{3} \sum_{j>i}^{3} \frac{1}{r_{i j}}+V_{c}\right) \Psi^{(0)} d^{3} \vec{r}
\end{aligned}
$$

Aprovechando la ortonormalidad de las funciones de espín

$$
\begin{aligned}
& \langle\alpha \mid \alpha\rangle=\langle\beta \mid \beta\rangle=1 \\
& \langle\alpha \mid \beta\rangle=\langle\beta \mid \alpha\rangle=0
\end{aligned}
$$

La ecuación (3.12) queda como

$$
\left\langle\Psi^{(0)}\left|H^{(0)}\right| \Psi^{(0)}\right\rangle=\left\langle\Psi_{1}\left|H^{(0)}\right| \Psi_{1}\right\rangle+\left\langle\Psi_{2}\left|H^{(0)}\right| \Psi_{2}\right\rangle+\left\langle\Psi_{3}\left|H^{(0)}\right| \Psi_{3}\right\rangle
$$

Aplicando el operador hamiltoniano a todos los términos del lado derecho de la ecuación (3.15), se encuentran diferentes tipos de integrales, tales como

-Las integrales de traslape

$$
S_{i j}=\int \phi_{i}^{*}(\vec{r}) \phi_{j}(\vec{r}) d^{3} \vec{r}
$$

-Las integrales de energía cinética

$$
T_{i j}=\frac{1}{2} \int \phi_{i}^{*}(\vec{r}) \nabla^{2} \phi_{j}(\vec{r}) d^{3} \vec{r}
$$

-Las integrales de energía potencial de interacción entre el núcleo y el electrón

$$
V_{i j}=\int \phi_{i}^{*}(\vec{r}) \frac{1}{r} \phi_{j}(\vec{r}) d^{3} \vec{r}
$$

-Las integrales bielectrónicas o de repulsión electrónica y de intercambio

$$
\begin{aligned}
& J_{i j}=\iint \phi_{i}^{*}\left(\vec{r}_{1}\right) \phi_{j}^{*}\left(\vec{r}_{2}\right) \frac{1}{r_{12}} \phi_{i}\left(\vec{r}_{1}\right) \phi_{j}\left(\vec{r}_{2}\right) d^{3} \vec{r}_{1} d^{3} \vec{r}_{2} \\
& K_{i j}=\iint \phi_{i}^{*}\left(\vec{r}_{1}\right) \phi_{j}^{*}\left(\vec{r}_{2}\right) \frac{1}{r_{12}} \phi_{i}\left(\vec{r}_{2}\right) \phi_{j}\left(\vec{r}_{1}\right) d^{3} \vec{r}_{1} d^{3} \vec{r}_{2}
\end{aligned}
$$

-E integrales de la forma 


$$
M_{2123}=\iint \phi_{2}^{*}\left(\vec{r}_{2}\right) \phi_{1}^{*}\left(\vec{r}_{3}\right) \frac{1}{r_{23}} \phi_{2}\left(\vec{r}_{2}\right) \phi_{3}\left(\vec{r}_{3}\right) d^{3} \vec{r}_{2} d^{3} \vec{r}_{3}
$$

Identificando el tipo de integral y agrupando términos, se llega a la expresión

$$
\begin{aligned}
\left\langle\Psi_{1}\left|H^{(0)}\right| \Psi_{1}\right\rangle & =2 S_{22} S_{33} H_{11}+2\left(S_{11} S_{33}-S_{13}^{2}\right) H_{22}+2 S_{11} S_{22} H_{33} \\
& -4 S_{22} S_{13} H_{13}+2 S_{33} J_{12}+2 S_{22} J_{13}+2 S_{11} J_{23}-2 S_{22} K_{13} \\
& -4 S_{13} M_{1232}
\end{aligned}
$$

Donde

$$
H_{i j}=-\left\langle\phi_{i}(\vec{r})\left|\frac{1}{2} \nabla^{2}\right| \phi_{j}(\vec{r})\right\rangle-\left\langle\phi_{i}(\vec{r})\left|\frac{Z}{r}\right| \phi_{j}(\vec{r})\right\rangle
$$

Contiene términos de energía cinética y energía potencial. Se obtienen resultados similares para las integrales $\left\langle\Psi_{2}\left|H^{(0)}\right| \Psi_{2}\right\rangle$ y $\left\langle\Psi_{3}\left|H^{(0)}\right| \Psi_{3}\right\rangle$. De esta forma, la expresión del valor esperado del hamiltoniano (3.15) puede escribirse como

$$
\begin{aligned}
& \left\langle\Psi^{(0)}\left|H^{(0)}\right| \Psi^{(0)}\right\rangle \\
& \quad=S_{22} S_{33} H_{11}+\left(S_{11} S_{33}-S_{13}^{2}\right) H_{22}+S_{11} S_{22} H_{33}-2 S_{22} S_{13} H_{13} \\
& \quad+S_{33} J_{12}+S_{22} J_{13}+S_{11} J_{23}-S_{22} K_{13}-2 S_{13} M_{1232}
\end{aligned}
$$

Así, la forma del funcional de la energía (3.11) para un sistema atómico confinado de tres electrones queda como

$$
\begin{aligned}
E^{(0)}=\left(\frac{1}{S_{11} S_{22} S_{33}-S_{22} S_{13}^{2}}\right)\left(S_{22} S_{33} H_{11}+\left(S_{11} S_{33}-S_{13}^{2}\right) H_{22}+S_{11} S_{22} H_{33}\right. \\
-2 S_{22} S_{13} H_{13}+S_{33} J_{12}+S_{22} J_{13}+S_{11} J_{23}-S_{22} K_{13} \\
\left.-2 S_{13} M_{1232}\right)
\end{aligned}
$$

Después de calcular todas las integrales implicadas en (3.26) e insertando su valor en dicha ecuación, sigue un proceso de minimización numérica respecto a los parámetros variacionales, dando previamente el valor de la carga nuclear $Z$ y el radio de confinamiento $R_{c}$, con el fin de determinar la energía del estado base del sistema.

En la Tabla 3.1 se muestra la energía del estado $1 s^{2} 2 s$ del átomo de litio obtenida por F. A. Duarte [65], como función del radio de confinamiento. Se puede apreciar que conforme el radio de confinamiento se hace más pequeño, la energía cinética de los electrones aumenta debido a la compresión. Esto hace que la energía del sistema pase de valores negativos a positivos, este cambio se presenta aproximadamente en $R_{c}=1.3241$ bohrs. 
Tabla 3.1. Cálculo variacional de la energía del estado $1 s^{2} 2 s$ del litio confinado dentro de una cavidad esférica impenetrable, como función del radio de confinamiento. Las energías están en hartrees y los radios en bohrs.

\begin{tabular}{|c|c|c|c|c|c|}
\hline$R_{C}$ & $\alpha$ & $\beta$ & $\gamma$ & $\delta$ & $E^{(0)}$ \\
\hline 0.4 & 4.4690 & 1.0774 & 8.8539 & 0.0839 & 138.2315 \\
\hline 1 & 2.2542 & 1.2927 & 4.4995 & 0.0089 & 8.324134 \\
\hline 1.3241 & 2.2073 & 1.4921 & 3.2151 & 1.1456 & -0.0001745 \\
\hline 1.5 & 2.24650 & 1.5986 & 2.6685 & 1.7036 & -2.17411 \\
\hline 2 & 2.31175 & 1.8832 & 1.7708 & 2.6068 & -5.1348 \\
\hline 3 & 2.23887 & 2.2217 & 1.1404 & 3.46917 & -6.7780 \\
\hline 4 & 2.44788 & 2.3689 & 0.95725 & 4.14868 & -7.18599 \\
\hline 5 & 2.49157 & 2.4454 & 0.89669 & 5.01366 & -7.323 \\
\hline 6 & 2.52346 & 2.4916 & 0.886798 & 6.12687 & -7.3769 \\
\hline 7 & 2.54739 & 2.5225 & 0.905472 & 7.23259 & -7.39969 \\
\hline 8 & 2.5659 & 2.5444 & 0.941717 & 7.809 & -7.40984 \\
\hline 9 & 2.5808 & 2.5611 & 0.986743 & 7.66173 & -7.41444 \\
\hline 10 & 2.5929 & 2.574 & 1.03296 & 7.14276 & -7.41658 \\
\hline 11 & 2.6029 & 2.5842 & 1.07532 & 6.60721 & -7.41762 \\
\hline 12 & 2.6112 & 2.5927 & 1.11171 & 6.18112 & -7.41815 \\
\hline 13 & 2.6183 & 2.5997 & 1.14204 & 5.86803 & -7.41846 \\
\hline 14 & 2.6243 & 2.6057 & 1.16709 & 5.64135 & -7.41864 \\
\hline 15 & 2.6295 & 2.6108 & 1.18783 & 5.4756 & -7.41876 \\
\hline$\infty$ & 2.6951 & 2.6765 & 1.38032 & 4.85168 & -7.41923 \\
\hline
\end{tabular}

\subsubsection{Estado $1 s^{2} 2 p_{z}$}

Para calcular la energía del estado $1 s^{2} 2 p_{z}$ del átomo de litio confinado dentro de una cavidad esférica de paredes impenetrables, se recurre al uso del método variacional directo, siguiendo exactamente la misma metodología que se mostró en la Sección 3.1.1, con las siguientes funciones hidrogenoides 


$$
\begin{gathered}
\phi_{1}(\vec{r})=N_{1} e^{-\alpha r}\left(1-\frac{r}{R_{c}}\right) Y_{00}(\theta, \varphi) \\
\phi_{2}(\vec{r})=N_{2} e^{-\beta r}\left(1-\frac{r}{R_{c}}\right) Y_{00}(\theta, \varphi) \\
\phi_{3}(\vec{r})=N_{3} r e^{-\gamma r / 2}\left(1-\frac{r}{R_{c}}\right) Y_{10}(\theta, \varphi)
\end{gathered}
$$

Donde $\alpha, \beta$ y $\gamma$ son parámetros variacionales y $N_{1}, N_{2}$ y $N_{3}$ son constantes de normalización.

En la Tabla 3.2 se muestra la energía del estado $1 s^{2} 2 p_{z}$ como función del radio de confinamiento, calculadas por [111]. Como en el caso del estado $1 s^{2} 2 s$, conforme el radio de confinamiento disminuye, la energía del sistema aumenta.

Como se pudo demostrar en el Capítulo 1, para sistemas hidrogenoides, un efecto del confinamiento espacial es romper la degeneración para estados que se encuentran en la misma capa con número cuántico principal $n$, haciendo que el estado de energía más baja sea aquel con momento angular $l$ más alto. Un efecto semejante ocurre en el caso de sistemas atómicos de tres electrones confinados: conforme el radio de confinamiento disminuye, la energía del estado $1 s^{2} 2 p_{z}$ se vuelve menor a la del estado $1 s^{2} 2 s$, es decir, el estado $1 s^{2} 2 p_{z}$ pasa a ser el estado base del sistema. Este fenómeno puede apreciarse en la Figura 3.1. 


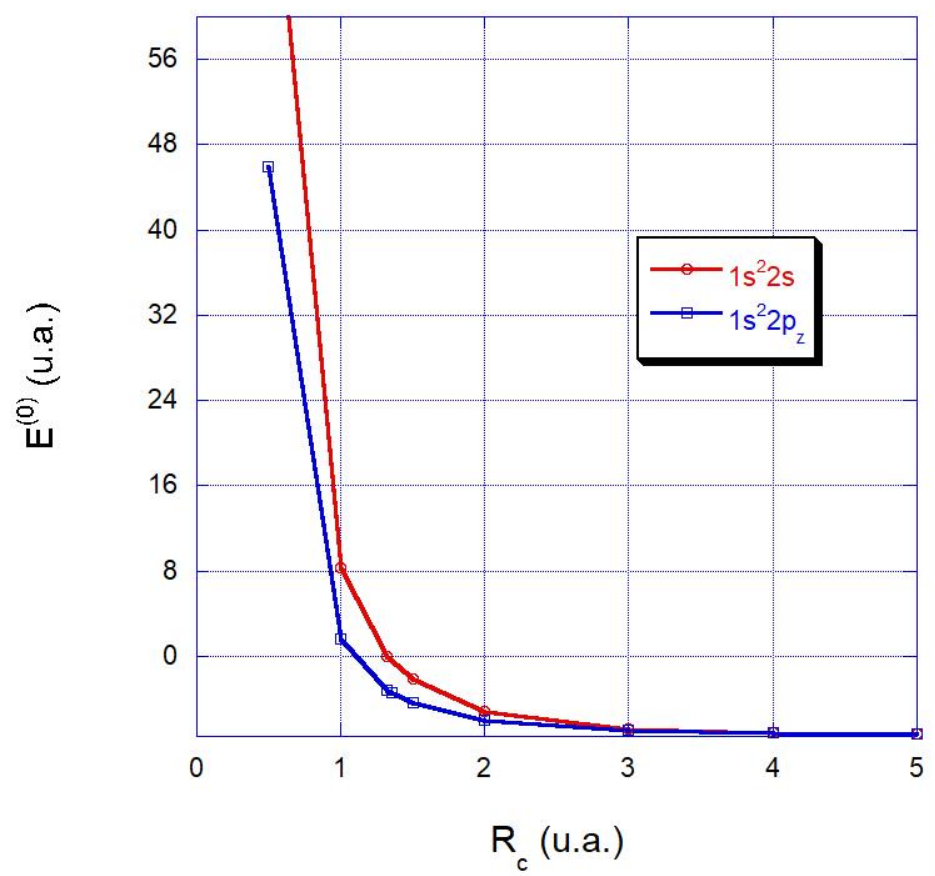

Figura 3.1. Energía $E^{(0)}$ de los estados $1 s^{2} 2 s$ y $1 s^{2} 2 p_{z}$ del átomo de litio confinado, como función del radio de confinamiento $R_{c}$.

\subsection{Corrección de núcleo de tamaño finito}

El hamiltoniano de un átomo de litio confinado dentro de un pozo esférico de paredes impenetrables, considerando al núcleo (anclado en el centro de la cavidad) como una distribución de carga esférica uniforme de carga $+Z e$, está dado por

$$
H=-\frac{1}{2} \sum_{i=1}^{3} \nabla_{i}^{2}+V\left(r_{1}, r_{2}, r_{3}\right)+V_{c}
$$

Donde el primer término de la izquierda corresponde a la energía cinética de los tres electrones. $V_{c}$ es el potencial de confinamiento (ecuación (3.2)) y $V\left(r_{1}, r_{2}, r_{3}\right)$ es el potencial de interacción electrostática entre el núcleo y los tres electrones 
Tabla 3.2. Parámetros variacionales $\alpha, \beta, \gamma$ y energía $E^{(0)}$ del estado $1 s^{2} 2 p_{z}$ del átomo de litio confinado, como función del radio de confinamiento $R_{c}$. Las energías están en hartrees y los radios en bohrs.

\begin{tabular}{|c|c|c|c|c|}
\hline$R_{c}$ & $\alpha$ & $\beta$ & $\gamma$ & $E^{(0)}$ \\
\hline 0.5 & 1.0931 & 1.1845 & 3.57175 & 45.8736 \\
\hline 1 & 1.27971091 & 1.4359323 & 2.29473297 & 1.655151561 \\
\hline 1.3241 & 1.42305 & 1.63386 & 1.98939 & -3.1771 \\
\hline 1.36 & 1.43973 & 1.65637 & 1.96429 & -3.47343 \\
\hline 1.5 & 1.50587532 & 1.74314294 & 1.87684018 & -4.387997323 \\
\hline 2 & 1.74488368 & 2.00775011 & 1.64497071 & -5.985165046 \\
\hline 3 & 2.11706606 & 2.2837313 & 1.31279753 & -6.879741566 \\
\hline 4 & 2.31320008 & 2.40143264 & 1.08247664 & -7.139919898 \\
\hline 5 & 2.41535671 & 2.46542226 & 0.9451917 & -7.246558483 \\
\hline 6 & 2.4741791 & 2.50588816 & 0.87043048 & -7.297107457 \\
\hline 7 & 2.51153583 & 2.53388162 & 0.83428041 & -7.322922215 \\
\hline 8 & 2.53712494 & 2.55442714 & 0.82254469 & -7.336670627 \\
\hline 9 & 2.55565515 & 2.5701583 & 0.82663815 & -7.344178501 \\
\hline 10 & 2.56964378 & 2.58259406 & 0.84066973 & -7.348353523 \\
\hline & & & & \\
\hline
\end{tabular}

$$
V\left(r_{1}, r_{2}, r_{3}\right)=\left\{\begin{array}{rr}
\frac{Z}{2 r_{0}}\left[\sum_{i=1}^{3}\left(\frac{r_{i}}{r_{0}}\right)^{2}-9\right]+\sum_{i=1}^{3} \sum_{j>i}^{3} \frac{1}{r_{i j}}, & r_{1}, r_{2}, r_{2} \leq r_{0} \\
-Z \sum_{i=1}^{3} \frac{1}{r_{i}}+\sum_{i=1}^{3} \sum_{j>i}^{3} \frac{1}{r_{i j}}, & r_{1}, r_{2}, r_{2} \geq r_{0}
\end{array}\right.
$$

$r_{0}$ es el radio del núcleo.

El hamiltoniano (3.29) se puede reescribir como 


$$
\begin{aligned}
H=\left(-\frac{1}{2} \sum_{i=1}^{3} \nabla_{i}^{2}-Z \sum_{i=1}^{3} \frac{1}{r_{i}}+\sum_{i=1}^{3} \sum_{j>i}^{3} \frac{1}{r_{i j}}+V_{c}\right) \\
+\left(V\left(r_{1}, r_{2}, r_{3}\right)+Z \sum_{i=1}^{3} \frac{1}{r_{i}}-\sum_{i=1}^{3} \sum_{j>i}^{3} \frac{1}{r_{i j}}\right)
\end{aligned}
$$

Donde se ha sumado y restado la cantidad

$$
Z \sum_{i=1}^{3} \frac{1}{r_{i}}-\sum_{i=1}^{3} \sum_{j>i}^{3} \frac{1}{r_{i j}}
$$

De esta manera, el hamiltoniano $H$ puede escribirse como

$$
H=H^{(0)}+H^{\prime}
$$

Donde $H^{(0)}$ es el hamiltoniano del átomo de litio confinado, considerando al núcleo como una partícula puntual (ecuación (3.1)). $H^{\prime}$ es la perturbación al sistema debida a considerar el tamaño finito del núcleo, y está dada por

$$
\begin{gathered}
H^{\prime}=V\left(r_{1}, r_{2}, r_{3}\right)+Z \sum_{i=1}^{3} \frac{1}{r_{i}}-\sum_{i=1}^{3} \sum_{j>i}^{3} \frac{1}{r_{i j}} \\
H^{\prime}=\left\{\begin{array}{rr}
\frac{Z}{2 r_{0}}\left[\sum_{i=1}^{3}\left(\frac{r_{i}}{r_{0}}\right)^{2}-9\right]+Z \sum_{i=1}^{3} \frac{1}{r_{i}}, & r_{1}, r_{2}, r_{3} \leq r_{0} \\
0, & r_{1}, r_{2}, r_{3}>r_{0}
\end{array}\right.
\end{gathered}
$$

Usando teoría de perturbaciones a primer orden, la corrección a la energía del átomo de litio confinado, debida a considerar un núcleo de tamaño finito, estará dada por

$$
E^{(1)}=\frac{\left\langle\Psi^{(0)}\left(\vec{r}_{1}, \vec{r}_{2}, \vec{r}_{3}\right)\left|H^{\prime}\right| \Psi^{(0)}\left(\vec{r}_{1}, \vec{r}_{2}, \vec{r}_{3}\right)\right\rangle}{\left\langle\Psi^{(0)}\left(\vec{r}_{1}, \vec{r}_{2}, \vec{r}_{3}\right) \mid \Psi^{(0)}\left(\vec{r}_{1}, \vec{r}_{2}, \vec{r}_{3}\right)\right\rangle}
$$

Aprovechando la condición de ortonormalidad de las funciones de espín, el valor esperado (3.37) queda como

$$
\left\langle\Psi^{(0)}\left|H^{\prime}\right| \Psi^{(0)}\right\rangle=\left\langle\Psi_{1}\left|H^{\prime}\right| \Psi_{1}\right\rangle+\left\langle\Psi_{2}\left|H^{\prime}\right| \Psi_{2}\right\rangle+\left\langle\Psi_{3}\left|H^{\prime}\right| \Psi_{3}\right\rangle
$$

Desarrollando el primer término de la derecha se obtiene

$$
\left\langle\Psi_{1}\left|H^{\prime}\right| \Psi_{1}\right\rangle=\frac{1}{3}\left[S_{22} S_{33} H_{11}^{\prime}+\left(S_{11} S_{33}-S_{13}^{2}\right) H_{22}^{\prime}+S_{11} S_{22} H_{33}^{\prime}-2 S_{22} S_{13} H_{13}^{\prime}\right]
$$


Donde

$$
\begin{gathered}
S_{i j}=\left\langle\phi_{i}(\vec{r}) \mid \phi_{j}(\vec{r})\right\rangle=\delta_{l l^{\prime}} \int_{0}^{R_{c}} R_{i}^{*}(r) R_{j}^{*}(r) r^{2} d r \\
H_{i j}^{\prime}=\int \phi_{i}^{*}(\vec{r}) \frac{Z}{2 r_{0}}\left[\left(\frac{r}{r_{0}}\right)^{2}-3+\frac{2 r_{0}}{r}\right] \phi_{j}(\vec{r}) d \vec{r} \\
=\delta_{l l^{\prime}} \delta_{m m^{\prime}} \int_{0}^{r_{0}} R_{i}^{*}(r) \frac{Z}{2 r_{0}}\left[\left(\frac{r}{r_{0}}\right)^{2}-3+\frac{2 r_{0}}{r}\right] R_{j}(r) r^{2} d r
\end{gathered}
$$

El término $\delta_{l l^{\prime}} \delta_{m m^{\prime}}$ aparece por la ortonormalidad de los armónicos esféricos. El mismo resultado se obtiene para $\left\langle\Psi_{2}\left|H^{\prime}\right| \Psi_{2}\right\rangle$ y $\left\langle\Psi_{3}\left|H^{\prime}\right| \Psi_{3}\right\rangle$. De manera que

$$
\left\langle\Psi^{(0)}\left|H^{\prime}\right| \Psi^{(0)}\right\rangle=S_{22} S_{33} H_{11}^{\prime}+\left(S_{11} S_{33}-S_{13}^{2}\right) H_{22}^{\prime}+S_{11} S_{22} H_{33}^{\prime}-2 S_{22} S_{13} H_{13}^{\prime}
$$

Por otro lado, la integral de normalización, aplicando la condición de ortonormalidad de las funciones de espín, está dada por

$$
\begin{aligned}
\left\langle\Psi^{(0)} \mid \Psi^{(0)}\right\rangle & =\left\langle\Psi_{1} \mid \Psi_{1}\right\rangle+\left\langle\Psi_{2} \mid \Psi_{2}\right\rangle+\left\langle\Psi_{3} \mid \Psi_{3}\right\rangle \\
& =S_{11} S_{22} S_{33}-S_{22} S_{13}^{2}
\end{aligned}
$$

Por tanto, la corrección a las energías del átomo de litio confinado, debida a considerar un núcleo de tamaño finito con una distribución de carga esférica uniforme, está dada por

$$
E^{(1)}=\frac{S_{22} S_{33} H_{11}^{\prime}+\left(S_{11} S_{33}-S_{13}^{2}\right) H_{22}^{\prime}+S_{11} S_{22} H_{33}^{\prime}-2 S_{22} S_{13} H_{13}^{\prime}}{S_{11} S_{22} S_{33}-S_{22} S_{13}^{2}}
$$

Se puede simplificar la integral (3.40). Dado que $r_{0} \ll R_{c}$, la extensión espacial de las funciones de onda es mucho mayor que la extensión espacial del núcleo. Por tanto, en la región $r<r_{0}$ podemos hacer la aproximación

$$
R_{i}(r) \approx R_{i}(0)
$$

De esta forma

$$
\begin{gathered}
H_{i j}^{\prime} \approx R_{i}^{*}(0) R_{j}(0) \delta_{l l^{\prime}} \delta_{m m^{\prime}} \int_{0}^{r_{0}} \frac{Z}{2 r_{0}}\left[\left(\frac{r}{r_{0}}\right)^{2}-3+\frac{2 r_{0}}{r}\right] r^{2} d r \\
H_{i j}^{\prime}=\frac{Z r_{0}^{2}}{10} R_{i}^{*}(0) R_{j}(0) \delta_{l l^{\prime}} \delta_{m m^{\prime}}
\end{gathered}
$$

En las Tablas 3.3 y 3.4 se muestran los resultados obtenidos para la corrección de núcleo de tamaño finito en las energías de los estados $1 s^{2} 2 s$ y $1 s^{2} 2 p_{z}$, respectivamente, para un núcleo 
de ${ }^{6} L i$, cuyo radio nuclear es $r_{0}=2.55 \mathrm{fm}$, y para un núcleo de ${ }^{7} L i\left(r_{0}=2.39 \mathrm{fm}\right)$ [83]. Como en los casos estudiados en capítulos anteriores para barreras impenetrables, a medida que el sistema se encuentra más confinado, la corrección de núcleo de tamaño finito es mayor, y crece de forma ilimitada.

La corrección de núcleo finito es mayor para el núcleo con un mayor radio, como puede apreciarse de la ecuación (3.46). Es decir, la corrección es mayor para el núcleo de ${ }^{6} \mathrm{Li}$.

Comparando la corrección de núcleo finito del estado $1 s^{2} 2 s$ con la del estado $1 s^{2} 2 p_{z}$, podría esperarse que, debido a que a cierto radio crítico de confinamiento el estado $1 s^{2} 2 p_{z}$ pasa a ser el estado de menor energía, a partir de dicho radio la corrección de núcleo finito debiera ser mayor para este estado, ya que se esperaría que el radio orbital promedio de dicho estado fuera más pequeño. Sin embargo, como puede apreciarse en la Figura 3.2, para un núcleo de ${ }^{7} L i$, y en la Figura 3.3, para un núcleo de ${ }^{6} L i$, la corrección de núcleo finito del estado $1 s^{2} 2 s$ permanece invariablemente por encima de la corrección para el estado $1 s^{2} 2 p_{z}$, independientemente del radio nuclear, al menos para los núcleos aquí utilizados. 
Tabla 3.3. Corrección de núcleo finito $E^{(1)}$ del estado $1 s^{2} 2 s$ del átomo de litio confinado para un núcleo de ${ }^{7} \mathrm{Li}\left(r_{0}=2.39 \mathrm{fm}\right)$ y un núcleo de ${ }^{6} \mathrm{Li}\left(r_{0}=2.55 \mathrm{fm}\right)$, como función del radio de confinamiento $R_{c}$. Las energías están en $10^{-8}$ hartrees y los radios en bohrs.

\begin{tabular}{|c|c|c|}
\hline$R_{c}$ & $E^{(1)}\left({ }^{7} L i\right)$ & $E^{(1)}\left({ }^{6} L i\right)$ \\
\hline 0.4 & 237.83936 & 270.75004 \\
\hline 1 & 33.04148 & 37.61354 \\
\hline 1.3241 & 17.51938 & 19.94359 \\
\hline 1.5 & 15.13372 & 17.22782 \\
\hline 2 & 11.89639 & 13.54252 \\
\hline 3 & 9.48858 & 10.80155 \\
\hline 4 & 9.81551 & 11.17371 \\
\hline 5 & 9.68868 & 11.02933 \\
\hline 6 & 9.63814 & 10.97179 \\
\hline 7 & 9.61636 & 10.94700 \\
\hline 8 & 9.60596 & 10.93516 \\
\hline 9 & 9.60411 & 10.93306 \\
\hline 10 & 9.60426 & 10.93323 \\
\hline 11 & 9.60491 & 10.93397 \\
\hline 12 & 9.60642 & 10.93569 \\
\hline 13 & 9.60781 & 10.93727 \\
\hline 14 & 9.60903 & 10.93866 \\
\hline 15 & 9.60990 & 10.93965 \\
\hline
\end{tabular}


Tabla 3.4. Corrección de núcleo finito $E^{(1)}$ del estado $1 s^{2} 2 p_{z}$ del átomo de litio confinado para un núcleo de ${ }^{7} L i\left(r_{0}=2.39 \mathrm{fm}\right)$ y un núcleo de ${ }^{6} L i\left(r_{0}=2.55 \mathrm{fm}\right)$, como función del radio de confinamiento $R_{c}$. Las energías están en $10^{-8}$ hartrees y los radios en bohrs.

\begin{tabular}{|c|c|c|}
\hline$R_{C}$ & $E^{(1)}\left({ }^{7} \mathrm{Li}\right)$ & $E^{(1)}\left({ }^{6} \mathrm{Li}\right)$ \\
\hline 0.5 & 50.73309 & 57.75318 \\
\hline 1 & 12.55673 & 14.29425 \\
\hline 1.3241 & 9.04850 & 10.30057 \\
\hline 1.36 & 8.86055 & 10.08661 \\
\hline 1.5 & 8.33624 & 9.48975 \\
\hline 2 & 7.89981 & 8.99293 \\
\hline 3 & 8.51515 & 9.69342 \\
\hline 4 & 8.96932 & 10.21043 \\
\hline 5 & 9.19168 & 10.46356 \\
\hline 6 & 9.30182 & 10.58895 \\
\hline 7 & 9.36023 & 10.65543 \\
\hline 8 & 9.39325 & 10.69302 \\
\hline 9 & 9.41278 & 10.71525 \\
\hline 10 & 9.42464 & 10.72875 \\
\hline & & \\
\hline
\end{tabular}




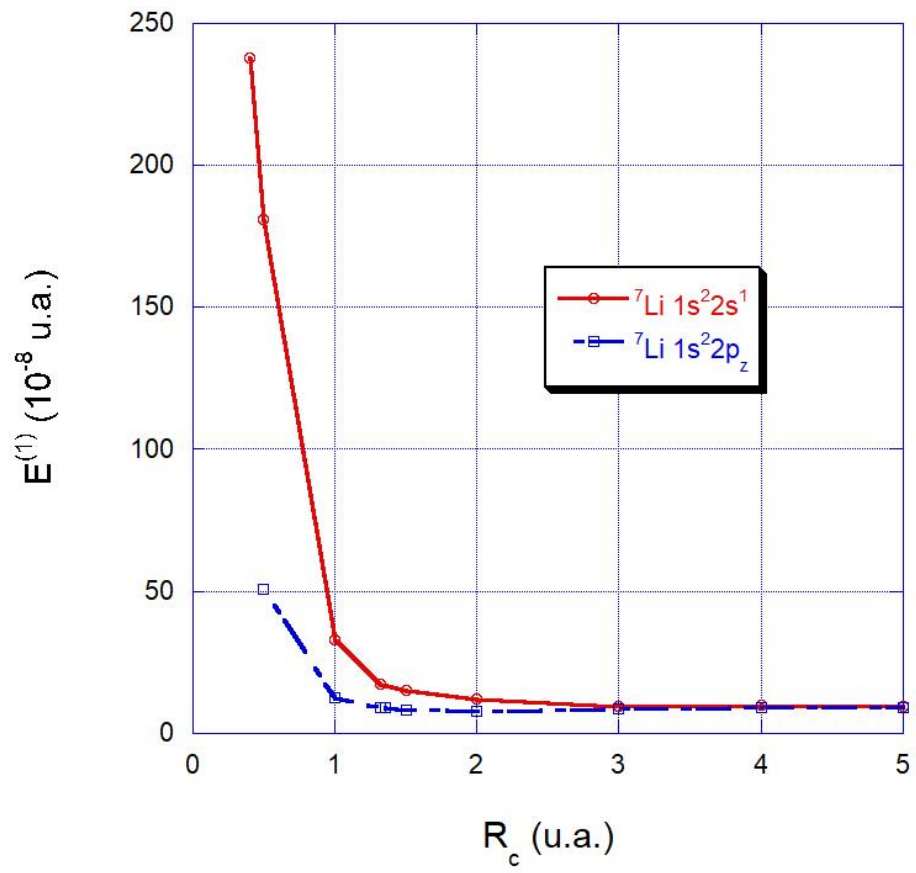

Figura 3.2. Corrección de núcleo finito a la energía de los estados $1 s^{2} 2 s$ y $1 s^{2} 2 p_{z}$ del átomo de litio confinado, como función del radio de confinamiento, para el isótopo ${ }^{7} \mathrm{Li}$.

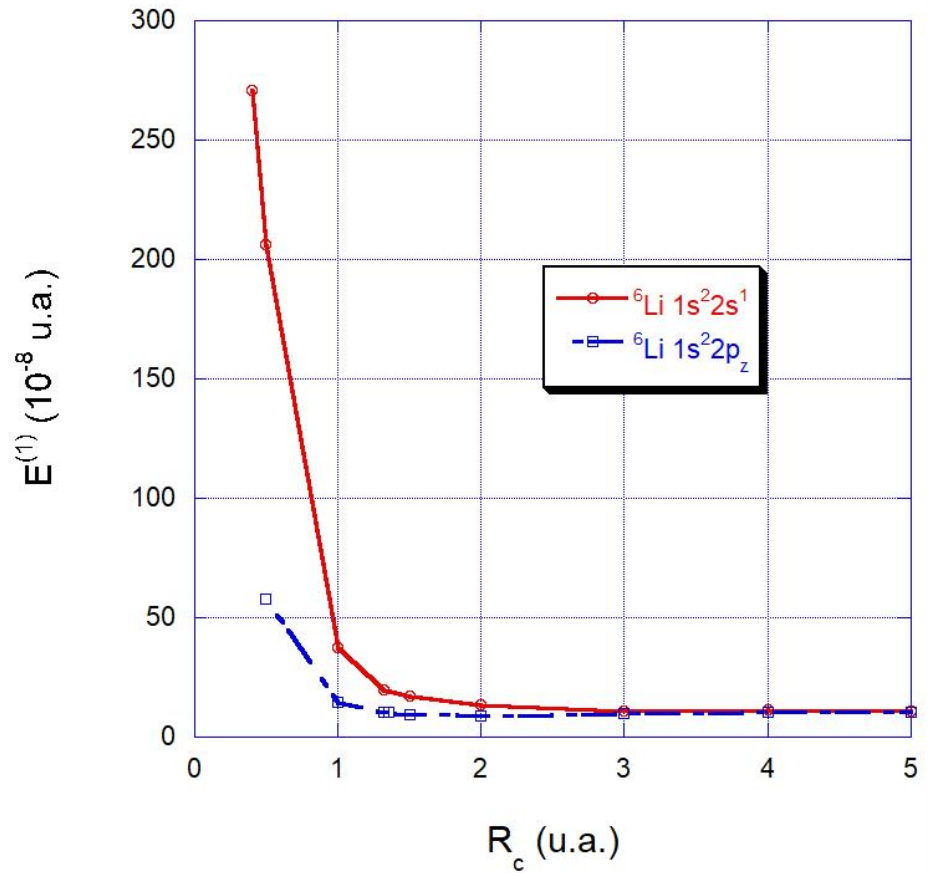

Figura 3.3. Corrección de núcleo finito a la energía de los estados $1 s^{2} 2 s$ y $1 s^{2} 2 p_{z}$ del átomo de litio confinado, como función del radio de confinamiento, para el isótopo ${ }^{6} \mathrm{Li}$. 


\subsection{Conclusiones}

Se calculó la corrección de núcleo de tamaño finito a la energía del estado base del átomo de litio confinado dentro de una barrera esférica de paredes impenetrables, como función del radio de confinamiento, utilizando teoría de perturbaciones independientes del tiempo a primer orden. Se consideró al núcleo como una distribución de carga esférica uniforme de radio $r_{0}$. Se demostró que a medida que el sistema se encuentra más confinado, la corrección de núcleo de tamaño finito se vuelve cada vez mayor, sin embargo del orden de $10^{-8}$ hartrees, muy pequeña comparada con los eigenvalores del sistema, por lo que el uso de teoría de perturbaciones sigue siendo válido.

Las energías del sistema no perturbado (considerando al núcleo como una partícula puntual) fueron calculadas por Duarte $[65,111]$ para los estados $1 s^{2} 2 s$ y $1 s^{2} 2 p_{z}$, usando el método variacional de Rayleigh-Ritz, con una función de prueba formada por un determinante de Slater con funciones hidrogenoides espín-orbitales.

Se encontró que uno de los efectos producidos por el confinamiento espacial del átomo es que, mientras que para el litio libre de confinamiento el estado base corresponde al estado $1 s^{2} 2 s$, a medida que el radio de confinamiento disminuye, la energía del estado $1 s^{2} 2 p_{z}$ pasa a ser la más baja, convirtiéndose este estado en el nuevo estado base del sistema. Aunque ocurra este efecto, la corrección de núcleo de tamaño finito continúa siendo mayor para el estado $1 s^{2} 2 s$, lo que sugiere que, en promedio, los electrones se hallan más cerca del núcleo en este estado que en el estado $1 s^{2} 2 p_{z}$, independientemente del confinamiento y el radio nuclear. 


\section{Capítulo 4}

\section{Átomos muónicos confinados}

Un átomo muónico se forma cuando se reemplaza un electrón de la capa más interna del átomo por un muón negativo $\mu^{-}$, cuya masa es $m_{\mu} \approx 207 m_{e}$, donde $m_{e}$ es la masa del electrón, y su carga es la misma que la de éste. Debido a su mayor masa, el muón, en comparación al electrón, se mueve a lo largo de una órbita mucho más cercana al núcleo, por lo que la interacción con los electrones de las capas exteriores del átomo es despreciable, y al sistema se le puede considerar como un átomo tipo hidrogenoide. Una órbita de menor envergadura conlleva, además, un aumento en la energía de enlace.

Como se estudió en capítulos anteriores, el corrimiento de las energías de un sistema atómico, debido a considerar el tamaño finito del núcleo, es varios órdenes de magnitud menor que las energías de Bohr del sistema (al menos para átomos de uno a tres electrones), incluso para sistemas confinados espacialmente.

Sin embargo, reemplazar al electrón por un muón, y por consiguiente al reducir el radio orbital promedio de la partícula alrededor del núcleo, sumado al confinamiento espacial del sistema, que reduce aún más la órbita promedio del muón, provoca que la interacción de éste con el potencial nuclear se intensifique, por lo que considerar la estructura interna del núcleo se vuelve más importante.

En este capítulo se estudia al átomo muónico confinado dentro de una cavidad esférica de paredes impenetrables por medio de dos modelos: en el primero, se asume al núcleo como una partícula puntual, en tanto que en el segundo se considera al núcleo como una distribución de carga esférica y uniforme, recurriendo a la teoría de perturbaciones independientes del tiempo a primer orden, para calcular el corrimiento de la energía como función del radio de confinamiento y el radio nuclear. 


\subsection{Modelo del átomo muónico confinado con núcleo puntual}

El hamiltoniano no perturbado del átomo muónico confinado dentro de una caja esférica impenetrable de radio $R_{c}$, cuyo núcleo se encuentra anclado al centro de la cavidad, está dado por

$$
H^{(0)}=-\frac{\hbar^{2}}{2 M_{\mu}} \nabla^{2}-\frac{Z e^{2}}{r}+V_{c}
$$

Donde

$$
M_{\mu}=\frac{m_{\mu} m_{n u c}}{m_{\mu}+m_{n u c}}
$$

Es la masa reducida del sistema. $V_{c}$ es el potencial de confinamiento impenetrable

$$
V_{c}= \begin{cases}0, & r \leq R_{c} \\ \infty, & r>R_{c}\end{cases}
$$

Ya que se está tratando con un potencial central, la función de onda $\psi_{n l m}^{(0)}$ puede separarse en una parte radial y una angular, la solución de esta última está dada por los armónicos esféricos $Y_{l m}(\theta, \varphi)$, como ya lo hemos visto en capítulos previos.

Claramente, este problema es completamente análogo al de un átomo hidrogenoide dentro de paredes esféricas impenetrables, que se estudió en la Sección 1.2.1, con la diferencia de que la masa reducida involucra la masa del muón (en lugar de la del electrón). Como en el Capítulo 1, la solución analítica de este problema está dada por

$$
R_{n l}^{(0)}=A e^{-\rho / 2} \rho^{l}{ }_{1} F_{1}(-\beta+l+1,2 l+2, \rho)
$$

Donde $A$ es una constante de normalización, ${ }_{1} F_{1}$ es la función hipergeométrica confluente y

$$
\rho=\frac{2 Z r}{a_{\mu} \beta}, \quad \beta^{2}=-\frac{Z^{2} e^{2}}{2 a_{\mu} E}, \quad a_{\mu}=\frac{\hbar^{2}}{M_{\mu} e^{2}}
$$

Donde $a_{\mu}$ es el radio de Bohr del átomo muónico. Como puede verse, la única diferencia en comparación al átomo hidrogenoide confinado es el reescalamiento causado por $a_{\mu}$ en los niveles de energía.

Imponiendo las condiciones de frontera de Dirichlet, los eigenvalores de la energía de este sistema de obtienen hallando las raíces de la siguiente ecuación

$$
R_{n l}^{(0)}\left(r=R_{c}\right)=0
$$

Cuya solución, de acuerdo a la ecuación (4.4), lleva a hallar las raíces de

$$
{ }_{1} F_{1}\left(-\beta+l+1,2 l+2, \rho_{c}\right)=0
$$


Donde

$$
\rho_{c} \equiv \frac{2 Z R_{c}}{a_{\mu} \beta}
$$

Fijando el número atómico $Z$, el radio de confinamiento $R_{c}$ y el momento angular $l$, la primera raíz de la ecuación (4.7) corresponde al estado de energía más bajo, y las raíces sucesivas a los estados excitados.

\section{2. Átomos muónicos confinados con núcleo de tamaño finito}

Considerando al núcleo de tamaño finito como una esfera de radio $r_{0}$, con una distribución de carga uniforme de magnitud $+Z e$, el hamiltoniano del átomo muónico confinado está dado por

$$
H=-\frac{\hbar^{2}}{2 M_{\mu}} \nabla^{2}+V(r)+V_{c}
$$

Donde $V(r)$ es el potencial de interacción electrostática entre el núcleo y la partícula $\mu^{-}$[79, 92, 93]

$$
V(r)=\left\{\begin{array}{c}
\frac{Z e^{2}}{2 r_{0}}\left[\left(\frac{r}{r_{0}}\right)^{2}-3\right], \quad 0 \leq r \leq r_{0} \\
-\frac{Z e^{2}}{r}, \quad r \geq r_{0}
\end{array}\right.
$$

Mientras que $V_{c}$ es el potencial de confinamiento (ecuación (4.3)).

Sumando y restando $Z e^{2} / r$, el hamiltoniano de la ecuación (4.9) puede reescribirse como

$$
H=\left(-\frac{\hbar^{2}}{2 M_{\mu}} \nabla^{2}-\frac{Z e^{2}}{r}+V_{c}\right)+\left(V(r)+\frac{Z e^{2}}{r}\right)=H^{(0)}+H^{\prime}
$$

Donde

$$
H^{(0)}=-\frac{\hbar^{2}}{2 M_{\mu}} \nabla^{2}-\frac{Z e^{2}}{r}+V_{c}
$$

Es el hamiltoniano no perturbado, el cual corresponde al átomo muónico confinado tomando al núcleo como una partícula puntual (Sección 4.1)

La perturbación $H^{\prime}$ debida al tamaño finito del núcleo está dada por

$$
H^{\prime}=V(r)+\frac{Z e^{2}}{r}=\left\{\begin{array}{c}
\frac{Z e^{2}}{2 r_{0}}\left[\left(\frac{r}{r_{0}}\right)^{2}-3\right]+\frac{Z e^{2}}{r}, 0 \leq r \leq r_{0} \\
0, r \geq r_{0}
\end{array}\right.
$$


Para calcular la corrección a la energía a primer orden, se recurre al uso de teoría de perturbaciones independientes del tiempo. De acuerdo a ésta, se tiene que la corrección a la energía $E_{n l}^{(1)}$ es

$$
\begin{aligned}
E_{n l}^{(1)} & =\left\langle\psi_{n l m}^{(0)}\left|H^{\prime}\right| \psi_{n l m}^{(0)}\right\rangle \\
& =\frac{Z e^{2}}{2 r_{0}} \int_{0}^{r_{0}}\left|R_{n l}^{(0)}(r)\right|^{2}\left[\left(\frac{r}{r_{0}}\right)^{2}-3+\frac{2 r_{0}}{r}\right] r^{2} d r
\end{aligned}
$$

Donde las funciones de onda $\psi_{n l m}^{(0)}$ son las soluciones del sistema no perturbado (Sección 4.1)

$$
\psi_{n l m}^{(0)}(r, \theta, \varphi)=R_{n l}^{(0)}(r) Y_{l m}(\theta, \varphi)
$$

Y las funciones radiales $R_{n l}^{(0)}$ están dadas por (4.4).

Las energías del sistema no perturbado fueron calculadas con el programa Mathematica, con una precisión de 30 cifras significativas, con un valor para el radio de Bohr del muón dado por

$$
a_{\mu}=\frac{m_{e}}{M_{\mu}} a_{0} \approx \frac{a_{0}}{186}
$$

Siendo $a_{0}$ el radio de Bohr del átomo de hidrógeno.

En las Tablas 4.1 a 4.4 se presentan las energías del sistema no perturbado y sus correspondientes correcciones de núcleo finito, como función del radio de confinamiento, para átomos muónicos con un núcleo de hidrógeno $\left(r_{0}=0.8335 \mathrm{fm}, m_{n u c}=\right.$ $\left.1.672621898 \times 10^{-27} \mathrm{Kg}\right) \quad[81]$, un núcleo de ${ }^{4} \mathrm{He}\left(r_{0}=1.5 \mathrm{fm}, m_{n u c}=\right.$ $\left.6.695098738000001 \times 10^{-27} \mathrm{Kg}\right)$ [82], y núcleos de los isótopos ${ }^{6} \mathrm{Li}\left(r_{0}=2.55 \mathrm{fm}\right.$, $\left.m_{n u c}=1.0042648107 \times 10^{-26} \mathrm{Kg}\right)$ y ${ }^{7} L i\left(r_{0}=2.39 \mathrm{fm}, m_{n u c}=1.1717575578 \times\right.$ $10^{-26} \mathrm{Kg}$ ) [83], respectivamente. 
Tabla 4.1. Energía del estado base $E_{10}$ y corrección de núcleo finito $E_{10}^{(1)}$, como función del radio de confinamiento $R_{c}$, para un átomo muónico confinado con núcleo de hidrógeno. Las energías están en keV y las distancias en fm.

\begin{tabular}{|c|c|c|}
\hline$R_{C}$ & $E_{10}$ & $E_{10}^{(1)}$ \\
\hline 142.373771794578 & 74.5490814892053 & 0.000949758759328 \\
\hline 170.848526153493 & 48.1613309617549 & 0.000587604456953 \\
\hline 199.323280512409 & 32.7046399671953 & 0.000395680188568 \\
\hline 227.798034871325 & 22.9661993246177 & 0.000283496963211 \\
\hline 256.272789230240 & 16.4899462820933 & 0.000212983888712 \\
\hline 284.747543589156 & 12.0002168547659 & 0.000166112093258 \\
\hline 355.934429486445 & 5.46484519406817 & 0.000100754224754 \\
\hline 427.121315383734 & 2.20906981037571 & 0.000069123399956 \\
\hline 498.308201281022 & 0.419650412562414 & 0.000051628595888 \\
\hline 569.495087178311 & -0.631858836638098 & 0.000041028794576 \\
\hline 711.868858972889 & -1.69292768114189 & 0.000029532557608 \\
\hline 854.242630767467 & -2.14309981759896 & 0.000023932439474 \\
\hline 996.616402562045 & -2.34726523968665 & 0.000020947122648 \\
\hline 1138.99017435662 & -2.44284361246862 & 0.000019290066353 \\
\hline 1281.36394615120 & -2.48803479196765 & 0.000018362373055 \\
\hline 1423.73771794578 & -2.50932377817794 & 0.000017849079125 \\
\hline 2847.47543589156 & -2.52743162253577 & 0.000017282591302 \\
\hline 5694.95087178311 & -2.52743534655236 & 0.000017282326589 \\
\hline$\infty$ & -2.52743534655239 & 0.000017282326589 \\
\hline
\end{tabular}


Tabla 4.2. Energía del estado base $E_{10}$ y corrección de núcleo finito $E_{10}^{(1)}$, como función del radio de confinamiento $R_{c}$, para un átomo muónico confinado con núcleo de ${ }^{4} H e$. Las energías están en $\mathrm{keV}$ y las distancias en fm.

\begin{tabular}{|c|c|c|}
\hline$R_{C}$ & $E_{10}$ & $E_{10}^{(1)}$ \\
\hline 131.563831279163 & 51.9448580770078 & 0.010831503737106 \\
\hline 157.876597534996 & 27.7736486925892 & 0.007177919209841 \\
\hline 184.189363790828 & 13.7726403706338 & 0.005122281312931 \\
\hline 210.502130046661 & 5.93653487705312 & 0.003976192704428 \\
\hline 236.814896302494 & 0.712357387274293 & 0.003201296758228 \\
\hline 263.127662558326 & -2.73510203949641 & 0.002675439005233 \\
\hline 394.691493837489 & -9.27675034687623 & 0.001560614056935 \\
\hline 526.255325116652 & -10.5742393066516 & 0.001257890139294 \\
\hline 592.037240756234 & -10.7698565553913 & 0.001197396170264 \\
\hline 657.819156395815 & -10.8620093373519 & 0.001163924754263 \\
\hline 789.382987674978 & -10.9245945938448 & 0.001136343818139 \\
\hline 920.946818954141 & -10.9374012426302 & 0.001129118572551 \\
\hline 1052.51065023330 & -10.9398633354125 & 0.001127422844580 \\
\hline 1184.07448151247 & -10.9403130687502 & 0.001127058083606 \\
\hline 1315.63831279163 & -10.9403920379840 & 0.001126984587883 \\
\hline 2631.27662558326 & -10.9404081579854 & 0.001126967326189 \\
\hline 5262.55325116652 & -10.9404081579856 & 0.001126967326188 \\
\hline$\infty$ & -10.9404081579856 & 0.001126967326188 \\
\hline
\end{tabular}


Tabla 4.3. Energía del estado base $E_{10}$ y corrección de núcleo finito $E_{10}^{(1)}$, como función del radio de confinamiento $R_{C}$, para un átomo muónico confinado con núcleo de ${ }^{6} \mathrm{Li}$. Las energías están en $\mathrm{keV}$ y las distancias en $\mathrm{fm}$.

\begin{tabular}{|c|c|c|}
\hline \multicolumn{1}{|c|}{$R_{c}$} & $E_{10}$ & $E_{10}^{(1)}$ \\
\hline 130.363829513888 & 21.7132499106660 & 0.066775811096209 \\
\hline 156.436595416666 & 1.61755796667995 & 0.047428151924454 \\
\hline 182.509361319443 & -9.11738607442037 & 0.036660954951966 \\
\hline 208.582127222221 & -15.2233901303265 & 0.030131584305707 \\
\hline 234.654893124998 & -18.8484806838073 & 0.025932728415087 \\
\hline 260.727659027776 & -21.0648218107307 & 0.023122052854835 \\
\hline 391.091488541664 & -24.4552349458062 & 0.017740743642863 \\
\hline 521.455318055552 & -24.8066003577754 & 0.016836198149698 \\
\hline 651.819147569440 & -24.8395865735743 & 0.016712071164147 \\
\hline 782.182977083328 & -24.8422924763124 & 0.016698620906596 \\
\hline 912.546806597216 & -24.8424934600017 & 0.016697386214204 \\
\hline 1042.91063611110 & -24.8425074107376 & 0.016697284598358 \\
\hline 1173.27446562499 & -24.8425083337010 & 0.016697276843435 \\
\hline 1303.63829513888 & -24.8425083926188 & 0.016697276283443 \\
\hline 2607.27659027776 & -24.8425083965134 & 0.016697276241907 \\
\hline 5214.55318055552 & -24.8425083965134 & 0.016697276241907 \\
\hline$\infty$ & -24.8425083965134 & 0.016697276241907 \\
\hline
\end{tabular}


Tabla 4.4. Energía del estado base $E_{10}$ y corrección de núcleo finito $E_{10}^{(1)}$, como función del radio de confinamiento $R_{C}$, para un átomo muónico confinado con núcleo de ${ }^{7} L i$. Las energías están en $\mathrm{keV}$ y las distancias en $\mathrm{fm}$.

\begin{tabular}{|c|c|c|}
\hline$R_{c}$ & $E_{10}$ & $E_{10}^{(1)}$ \\
\hline 130.020769482651 & 21.7705403591248 & 0.059212041059470 \\
\hline 156.024923379181 & 1.62182589624832 & 0.042055669555096 \\
\hline 182.029077275711 & -9.14144231377305 & 0.032508039707315 \\
\hline 208.033231172241 & -15.2635570722269 & 0.026718262518204 \\
\hline 234.037385068771 & -18.8982124335723 & 0.022995031261639 \\
\hline 260.041538965301 & -21.1204013804960 & 0.020502740040800 \\
\hline 390.062308447952 & -24.5197601266508 & 0.015731021054745 \\
\hline 520.083077930602 & -24.8720526168836 & 0.014928943573976 \\
\hline 650.103847413253 & -24.9051258668716 & 0.014818877921726 \\
\hline 780.124616895903 & -24.9078389091388 & 0.014806951337922 \\
\hline 910.145386378554 & -24.9080404231240 & 0.014805856514310 \\
\hline 1040.16615586120 & -24.9080544106688 & 0.014805766409751 \\
\hline 1170.18692534385 & -24.9080553360675 & 0.014805759533324 \\
\hline 1300.20769482651 & -24.9080553951407 & 0.014805759036770 \\
\hline 2600.41538965301 & -24.9080553990456 & 0.014805758999939 \\
\hline 5200.83077930602 & -24.9080553990456 & 0.014805758999939 \\
\hline$\infty$ & -24.9080553990456 & 0.014805758999939 \\
\hline
\end{tabular}

Como puede observarse de estas Tablas, entre más confinado se encuentra el sistema, mayores son las correcciones a la energía del estado base debidas al tamaño finito del núcleo; sin embargo, al menos para los núcleos aquí estudiados, estas correcciones son algunos órdenes de magnitud más pequeñas que las energías del sistema no perturbado. Dado esto, la aplicación de teoría de perturbaciones a primer orden es válida. Por otro lado, conforme el radio de la cavidad aumenta, y por tanto la compresión del sistema disminuye, las energías tienden a sus valores correspondientes a su contraparte libre de confinamiento. 
La corrección a la energía debida al núcleo finito es mayor para núcleos más pesados, como se muestra en la Figura 4.1. En contraste, el efecto del confinamiento sobre la energía, en comparación con la del sistema no comprimido, es mayor para el sistema con el núcleo más ligero, como puede apreciarse en la Figura 4.2.

(a)

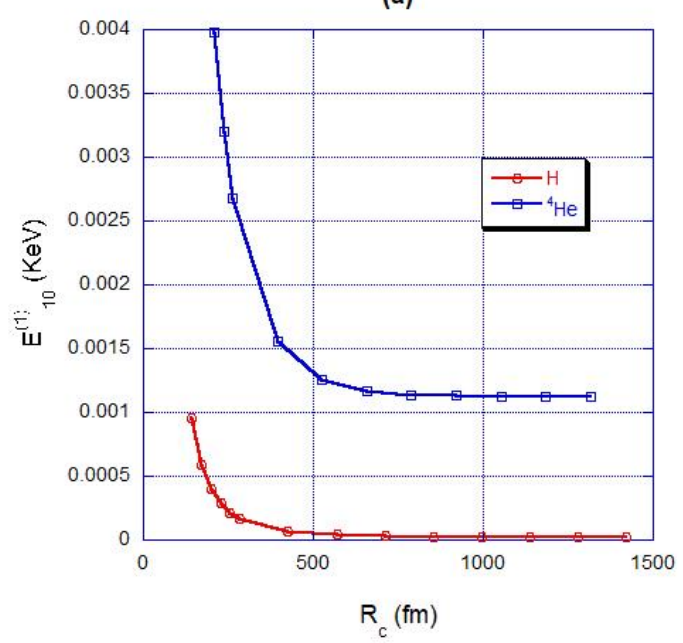

(b)

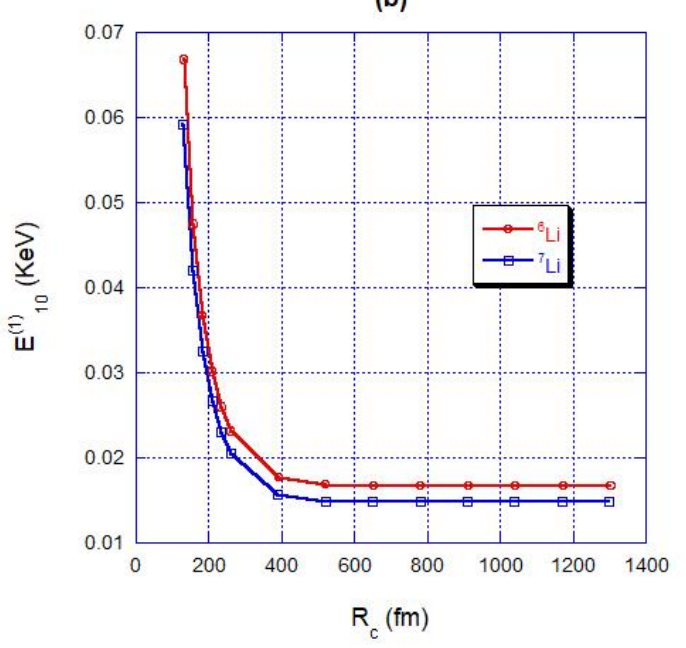

Figura 4.1. Corrección de núcleo finito a la energía del estado base del átomo muónico confinado dentro de una barrera impenetrable de radio $R_{c}$, como función del radio de confinamiento.

\subsection{Conclusiones}

Se calculó de forma exacta el espectro de energías del átomo muónico confinado dentro de una barrera esférica de paredes impenetrables. Dichos cálculos se efectuaron primero asumiendo al núcleo como una partícula puntual, y después, usando teoría de perturbaciones a primer orden, se obtuvieron correcciones para la energía del estado base debidas a los efectos inducidos al considerar el tamaño finito del núcleo, con una carga distribuida uniformemente en una esfera. Para estos sistemas se encontró que la corrección a la energía aumenta conforme el radio de confinamiento disminuye, y con el aumento en el radio del núcleo. Sin embargo, el efecto del confinamiento sobre estas correcciones, en comparación con el sistema libre de confinamiento, resulta ser mayor para los núcleos de menor tamaño. 
Esto es probablemente debido a que, entre más ligado se encuentra el muón al núcleo, éste siente menos los efectos de la barrera de confinamiento.

Por otro lado, usar teoría de perturbaciones para estimar las correcciones de núcleo de tamaño finito, no se justifica para átomos muónicos libres de confinamiento con núcleos pesados, ya que se ha observado que en esos casos la probabilidad de hallar al muón dentro del núcleo es alta [92]. Es de esperar un comportamiento similar en el caso de que estos sistemas sean sometidos a altas presiones externas.

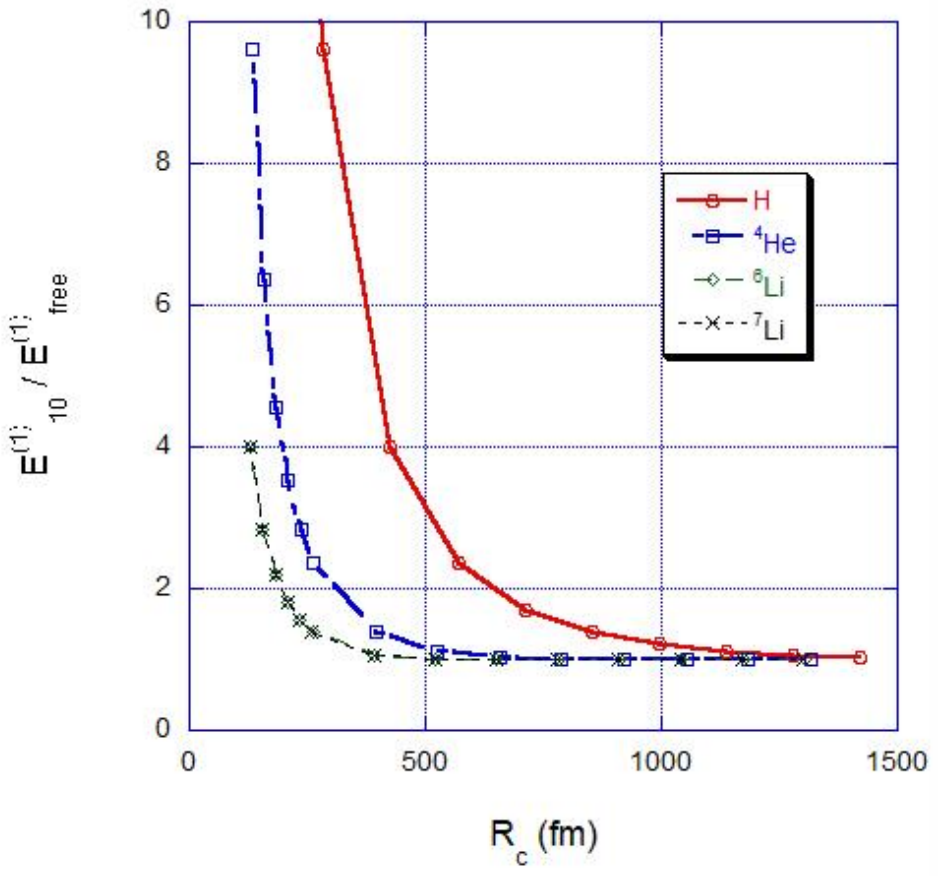

Figura 4.2. Razón de la corrección de núcleo finito del átomo muónico confinado, $E_{10}^{(1)}$, y la corrección de núcleo finito del átomo muónico libre de confinamiento, $E_{f r e e}^{(1)}$. 


\section{Conclusiones generales}

Se estudió el comportamiento de algunos sistemas atómicos de uno, dos y tres electrones, así como átomos muónicos, sometidos a altas presiones externas, haciendo uso de un modelo de confinamiento espacial dentro de una barrera de potencial con simetría esférica, tanto de paredes penetrables como impenetrables.

En el caso del átomo de hidrógeno confinado dentro de una barrera de paredes impenetrables, se resolvió este problema usando el método variacional de Rayleigh-Ritz, con funciones de prueba que incluían diferentes funciones de corte, para asegurar el cumplimiento de las condiciones de frontera de Dirichlet.

Al menos para el estudio de dicho problema, se demostró que la conjetura de Gadre, la cual sugiere que la función de onda con la entropía de Shannon más alta de un conjunto de funciones es la de mayor calidad, no es adecuada, ya que las propiedades y valores esperados calculados en este trabajo para las diferentes funciones de prueba mostraron resultados muy variados, independientemente del valor de su entropía de Shannon.

Sólo se calculó la entropía informática de Shannon en el espacio de coordenadas, pero ya que el objetivo principal de esta tesis era un estudio general de las propiedades físicas de los sistemas atómicos confinados, la entropía de Shannon proveyó información suficiente acerca de la localización o deslocalización de las partículas en la región de confinamiento.

También se emplearon las energías y funciones propias exactas del átomo de hidrógeno confinado, con el fin de obtener mediante teoría de perturbaciones el espectro de estructura fina del AHC.

En este trabajo de tesis también se obtuvieron de forma exacta las funciones y energías propias del átomo de hidrógeno confinado dentro de paredes penetrables, por lo que se están 
dando las bases para el cálculo del espectro de estructura fina de este sistema, así como cualquier otra propiedad física que se desee calcular con alta precisión numérica.

En el caso de los átomos de helio y litio confinados, se calculó la energía del estado base de estos sistemas mediante el método variacional de Rayleigh-Ritz, con funciones de prueba relativamente simples, que dan una buena aproximación a la energía propia. Con estas funciones de prueba, también se calcularon algunas propiedades físicas.

Las correcciones a la energía de estos sistemas atómicos debidas al tamaño finito del núcleo resultan ser muy pequeñas y prácticamente despreciables. En este trabajo de tesis se calculó también la corrección de núcleo de tamaño finito para el átomo muónico confinado, con los tres núcleos estables más pequeños existentes en la naturaleza. Aunque en este caso la corrección a la energía es mayor que para los átomos multielectrónicos, sigue siendo varios órdenes de magnitud menor que las energías de Bohr del sistema.

Con respecto a las contribuciones del núcleo de tamaño finito a la energía de los sistemas atómicos confinados, a lo largo de este trabajo de tesis se consideró para los cálculos realizados que el núcleo se trata de una esfera cuya carga eléctrica se encuentra distribuida uniformemente en todo su volumen.

También pudo observarse que los efectos del confinamiento en átomos muónicos son apreciables sólo para radios de la caja demasiado pequeños (del orden de cientos de fm).

En esta tesis, se consideró un modelo de confinamiento en el que el núcleo se encuentra anclado al centro de la caja. En el caso de átomos multielectrónicos, esta aproximación es válida, ya que la masa del núcleo es mucho mayor que la de los electrones; para átomos muónicos, esta diferencia es menor, sin embargo todavía se puede considerar esta aproximación como adecuada, sobre todo para átomos con núcleos pesados.

En conclusión, a pesar de que han pasado más de 80 años desde los primeros estudios referentes a los sistemas cuánticos confinados, aún existen muchos tópicos poco estudiados, y de mucho interés para los investigadores, debido a la amplia variedad de aplicaciones de estos sistemas en la ciencia y la tecnología. 


\section{Perspectivas}

Los resultados aquí presentados respecto al estudio de las funciones de corte en el AHC les pueden ser de utilidad a otros autores cuya finalidad sea el estudio de sistemas cuánticos confinados dentro de paredes impenetrables, al momento de elegir una función adecuada para su investigación.

El método variacional directo tiene enormes aplicaciones en la física cuántica, específicamente en el caso de los sistemas atómicos confinados, como se ha mostrado en esta tesis. Además del confinamiento en tres dimensiones, se puede aplicar, por ejemplo, en el estudio de centros de color, modelados como puntos cuánticos [112].

Cabe señalar que en este trabajo sólo se analizó la entropía informática de Shannon en el espacio de configuraciones. Un estudio más completo de entropías de la información en sistemas atómicos y moleculares requiere, por ejemplo, del cálculo de la entropía de Shannon en el espacio de momentos, y de la entropía de Fisher, la cual provee de más información acerca de la agudeza de la distribución de probabilidad en cierta región del espacio.

Sistemas cuánticos confinados tales como átomos y moléculas atrapados dentro de cajas impenetrables, pozos y puntos cuánticos, son sistemas ideales para analizar el concepto de localización-deslocalización. En analogía al significado clásico de entropía, relacionado con el grado de desorden de un sistema, la entropía de Fisher está relacionada con el concepto de orden o complejidad [113].

Como se ha mencionado a lo largo de esta tesis, el uso de paredes impenetrables sobreestima el efecto del confinamiento, por lo que para trabajos futuros se desean calcular las correcciones relativistas del átomo de hidrógeno confinado, así como de otros sistemas atómicos, como el helio, con un modelo de confinamiento dentro de paredes penetrables. 
Los resultados obtenidos para el helio y para el litio se pueden mejorar eligiendo una función de onda con más parámetros variacionales, o utilizando un método de aproximación más refinado, como Hartree-Fock o Teoría de Funcionales de la Densidad (DFT).

Para el átomo de litio confinado, entre las propiedades que se pueden calcular, están sus potenciales de ionización, así como la entropía informática de Shannon.

En el caso de los átomos muónicos confinados, se pudo apreciar que, conforme el tamaño del núcleo aumenta, también lo hace la corrección del núcleo de tamaño finito, por lo que para el estudio de núcleos más pesados se requiere del uso de otros métodos de solución de la ecuación de Schrödinger, ya que el método de Teoría de Perturbaciones dejará de ser válido. Aun así, el método que se utilizó para calcular las energías de este sistema considerando al núcleo como una partícula puntual (en términos de la función hipergeométrica confluente), puede aplicarse a cualquier sistema hidrogenoide confinado, como el deuterio y el tritio, además de diversos iones.

Aunque el modelo del núcleo como una distribución de carga uniforme con simetría esférica es bueno para realizar una primera aproximación a la contribución del núcleo de tamaño finito a las energías del sistema, existen modelos nucleares más complejos que están más acordes con lo observado experimentalmente, y que por tanto serían de utilidad para obtener una mejor aproximación a la corrección de núcleo finito.

En los sistemas estudiados en esta tesis, siempre se consideró que el núcleo del átomo se encontraba anclado al centro del potencial de confinamiento. Sin embargo, si se consideran otros tipos de átomos exóticos, como los positronios (sistema formado por un electrón y su antipartícula, el positrón) dado que la masa de estas partículas es idéntica, no es apropiado hacer la aproximación del núcleo anclado al centro, ya que ambas partículas se estarán moviendo dentro de todo el volumen de confinamiento. Esto sucede de igual manera para cualquier sistema de dos partículas cuyas masas sean del mismo orden de magnitud o muy similares entre sí. 


\section{Bibliografía}

[1] A. Michels, J. de Boer and A. Bijl, Physica 4 (1937) 981.

[2] F. M. Fernández and E. A. Castro, Kinam 4 (1982) 193.

[3] B. Zhu and Y. C. Chang, Phys. Rev. B 50 (1994).

[4] P. Harrison, "Quantum Wells, Wires and Dots: Theoretical and Computational Physics of Semiconductor Nanostructures", 2a Ed., John Wiley and Sons, Ltd. (2005).

[5] M. López López y M. A. Meléndez Lira, "Pozos, Hilos y Puntos Cuánticos: Estructuras Semiconductoras de Baja Dimensión”, Avance y Perspectiva vol. 16 (1997).

[6] A. J. Chiquito, Rev. Bras. Ensino Fis. 23 (2001) 159.

[7] B. Çakır, A. Özmen and Y. Yakar, Physica B 481 (2016) 32-37.

[8] S. T. Pérez-Merchancano, L. E. Bolivar-Marinez y J. Silvia-Valencia, Rev. Mex. Fís. 53 (2007) 470-474.

[9] J. P. Connerade and P. Kengkan, Proc. Idea-Finding Symposium (2003) 35-46.

[10] J. Hernández-Rojas, J. Bretón and J. M. Gomez Llorente, J. Chem. Phys. 104 (1996) 1179.

[11] E. M. Corson and I. Kaplan, Phys. Rev. 71 (1947) 130.

[12] S. Goldman and C. Joslin, J. Phys. Chem. 96 (1992) 6021.

[13] M. Friedman, A. Rabinovitch and R. Thieberger, J. Comp. Phys. 33 (1979) 359.

[14] R. J. Swenson and S. H. Danforth, J. Chem. Phys. 57 (1972) 1734.

[15] J. P. Connerade and V. K. Dolmatov, J. Phys. B: At. Mol. Opt. Phys. 48 (2015) 015007.

[16] W. Harneit, Phys. Rev. A 65 (2002) 032322.

[17] N. Aquino, Advances in Quantum Chemistry 57 (2009) 123.

[18] A. Sommerfeld and H. Welker, Ann. Physik 32 (1938) 56.

[19] S. R. de Groot and C. A. ten Seldam, Physica XII (1946) 669.

[20] E. P. Wigner, Phys. Rev. 94 (1954) 77. 
[21] B. F. Gray, J. Chem. Phys. 36 (1962) 1801.

[22] B. F. Gray, J. Chem. Phys. 55 (1971) 2848.

[23] V. C. Aguilera-Navarro, W. M. Kloet and A. H. Zimermann, Rev. Brasil Fis. 1 (1971) 55.

[24] B. F. Gray and I. Gonda, J. Chem. Phys. 62 (1975) 2007.

[25] J. Gorecki and W. Byers Brown, J. Phys. B: At. Mol. Opt. Phys. 20 (1987) 5953.

[26] J. Gorecki and W. Byers-Brown, J. Phys. B: At. Mol. Opt. Phys. 21 (1988) 403.

[27] J. Gorecki and W. Byers Brown, J. Phys. B: At. Mol. Opt. Phys. 22 (1989) 2659.

[28] J. L. Marín and S. A. Cruz, J. Phys. B: At. Opt. Phys. 24 (1991) 2899-2907.

[29] J. L. Marín and S. A. Cruz, J. Phys. B: At. Opt. Phys. 25 (1992) 4365-4371.

[30] E. V. Ludeña, J. Chem. Phys. 66 (1977) 468.

[31] E. Ley-Koo and S. Rubinstein, J. Chem. Phys. 71 (1979) 351.

[32] V. C. Aguilera-Navarro, E. Ley-Koo and A. H. Zimerman, Rev. Brasil Fis. 10 (1980) 2.

[33] N. Aquino, Int. J. Quantum Chem. 54 (1995) 107.

[34] N. Aquino, J. Phys. A: Math. Gen. 30 (1997) 2403.

[35] N. Aquino, G. Campoy and H. E. Montgomery, Int. J. Quantum Chem., 107 (2007) 1548-1558.

[36] M. M. Almeida, M. N. Guimarães and F. V. Prudente, Rev. Brasil Fis. 27 (2005) 395405.

[37] D. Djajaputra and B. R. Cooper, Eur. J. Phys. 21 (2000) 261.

[38] S. V. Nair, S. Sinha, K. C. Rustagi, Phys. Rev. B. 35 (1987).

[39] D. Baye and K. D. Sen, Phys. Rev. E 78 (2008) 026701.

[40] J. Hunt, J. Martin, V. Rosing, J. Winner and H. E. Montgomery Jr., Chem. Educator 19 (2014) 394-389.

[41] A. Solórzano, N. Aquino and A. Flores-Riveros, Can. J. Phys. 94 (2016) 894-901.

[42] N. Aquino, A. Flores-Riveros and J. F. Rivas-Silva, Phys. Lett. A 377 (2013) 20622068.

[43] L. G. Jiao, L. R. Zan, Y. Z. Zhang and Y. K. Ho, Int. J. Quantum Chem. 117 (2017) $\mathrm{e} 25375$.

[44] R. Cabrera-Trujillo and S. A. Cruz, Phys. Rev. A 87 (2013) 012502. 
[45] N. Aquino and R. A. Rojas, Eur. J. Phys. 37 (2016) 015401.

[46] R. A. Rojas and N. Aquino, Rev. Mex. Fis. (2018). Trabajo aceptado

[47] H. E. Montgomery Jr. and K. D. Sen, Phys. Lett. A 376 (2012) 1992-1996.

[48] R. A. Rojas, "Métodos de solución al problema del átomo de hidrógeno confinado" (Tesis de Maestría), Universidad Autónoma Metropolitana, Ciudad de México, México (2014).

[49] R. M. Yu, L. R. Zan, L. G. Jiao and Y. K. Ho, Few Body Syst. 58 (2017) 152.

[50] E. Ley-Koo and S. Rubinstein, J. Chem. Phys. 73 (1980) 887.

[51] X. L. Yang, S. H. Guo, F. T. Chan, K. W. Wong and W. Y. Ching, Phys. Rev. A 43 (1991) 1186.

[52] N. Aquino and E. Castaño, Revista Mexicana de Física E 51 (2) (2005) 126-131.

[53] K. D. Sen, J. Garza, R. Vargas and N. Aquino, Phys. Lett. A 295 (2002) 299-304.

[54] E. Ley-Koo, S.A. Cruz, J. Chem. Phys. 74 (1981) 4603.

[55] J. P. Connerade, A. G. Lyalin, R. Semaoune, S. K. Semenov, A. V. Solov'yov, J. Phys. B: At. Opt. Phys. 34 (2001) 2505.

[56] A. Flores-Riveros, N. Aquino, and H. E. Montgomery Jr., Phys. Lett. A 374 (2010) 1246-1252.

[57] C. Le Sech and A. Banerjee, J. Phys. B: At. Mol. Opt. Phys. 44 (2011) 105003.

[58] X. Wen-Fang, Commun. Theor. Phys. 48 (2007) 331-334.

[59] T. D. Young, R. Vargas and J. Garza, Phys. Lett. A 380 (2016) 712-717.

[60] N. Aquino, J. Garza, A. Flores-Riveros, J. F. Rivas-Silva and K. D. Sen, J. Chem. Phys. 124 (2006) 054311.

[61] A. Banerjee, C. Kamal and A. Chowdhury, Phys. Lett. A 350 (2006) 121-125.

[62] C. Díaz-García and S. A. Cruz, Phys. Lett. A 353 (2006) 332-336.

[63] B. M. Gimarc, J. Chem. Phys. 47 (1967) 5110.

[64] C. A. Ten Seldam and S. R. de Groot, Physica 18 (1952) 905.

[65] F. A. Duarte, "Estudio de sistemas cuánticos confinados de tres electrones usando el método variacional directo y DFT" (Tesis de Maestría), Universidad de Sonora, Hermosillo, México (2013).

[66] A. Sarsa, E. Buendía and F. J. Gálvez, J. Phys. B: At. Mol. Opt. Phys. 47 (2014) 185002. 
[67] J. Garza, R. Vargas and A. Vela, Phys. Rev. E 58 (1998) 3949.

[68] E. V. Ludeña, J. Chem. Phys. 69 (1978) 1770.

[69] M. Rodríguez-Bautista, C. Díaz-García, A. M. Navarrete-López, R. Vargas and J. Garza, J. Chem. Phys. 143 (2015) 034103.

[70] J. Garza, J. M. Hernández-Pérez, J. Z. Ramírez and R. Vargas, J. Phys. B: At. Mol. Opt. Phys. 45 (2012) 015002.

[71] C. Díaz-García, "Efectos de confinamiento espacial en átomos multielectrónicos" (Tesis de Doctorado), Universidad Autónoma Metropolitana, Ciudad de México, México (2009).

[72] J. Gorecki and W. Byers-Brown, J. Chem. Phys. 89 (1988) 2138.

[73] E. Ley-Koo and S. A. Cruz, J. Chem. Phys. 74 (1981) 4603.

[74] D. Baye and J. Dohet-Eraly, Phys. Chem. Chem. Phys. 17 (2015) 31417.

[75] K.Bakke, Phys. Lett. A 374 (2010) 4642-4646.

[76] K. Bakke and C. Furtado, Phys. Lett. A 376 (2012) 1269-1273.

[77] V. Krápek, P. Klenovský and T. Sikola, Phys. Rev. B 92 (2015) 195430.

[78] U. Rössler, S. Jorda and D. Broido, Solid State Communications 73 (1990) 209-214.

[79] C. Cohen-Tannoudji, Quantum Mechanics, New York: John Wiley and sons Inc. (1977).

[80] A. A. Sokolov, Y. M. Loskutov and I. M. Ternov, Quantum Mechanics, New York: Holt Rinehart and Winston Inc. (1966).

[81] A. Beyer, L. Maisenbacher, A. Matveev, R. Pohl, K. Khabarova, A. Grinin, T. Lamour, D. C. Yost, T. W. Hänsch, N. Kolachevsky and T. Udem, Science 358 (2017) 79.

[82] T. N. Antsygina and K. A. Chishko, Low Temperature Physics 42 (2016) 734.

[83] Z. C. Yan and G. W. F. Drake, Phys. Rev. A 61 (2000) 225041-225048.

[84] G. W. F. Drake, W. Nörtershaüser and Z. C. Yan, Can. J. Phys. 83 (2005) 311.

[85] T, Hannesson and S. M. Blinder, Int. J. Quantum Chem. 15 (1979) 7-14.

[86] D. Andrae, Phys. Rep. 336 (2000) 4313-525.

[87] D. Andrae, M. Reiher and J. Hinze, Chem. Phys. Lett. 320 (2000) 457-468.

[88] P. J. Mohr, G. Plunien and G. Soff, Phys. Rep. 293 (1998) 227-369.

[89] L. I. Schiff, H. Snyder and J. Weinberg, Phys. Rev. 57 (1940) 315-318.

[90] I. Pomeranchuk and J. Smorodinsky, J. Phys. (Moscow) 9 (1945) 97-100. 
[91] I. P. Grant and H. M. Quiney, Adv. At. Mol. Phys. 23 (1987) 37-86.

[92] E. Ley-Koo, E. Castaño, D. Finotello, E. Nahmad-Achar and S. Ulloa, Am. J. Phys. 48 (1980) 949.

[93] B. C. Tiburzi and B. R. Holstein, Am. J. Phys. 68 (2000) 640.

[94] W. Demtröder, Atoms, Molecules and Photons: An Introduction to Atomic-, Molecular- and Quantum-Physics, Berlín: Springer (2006).

[95] C. E. Shannon, Bell Syst. Tech. J. 27 (1948) 379.

[96] S. R. Gadre, S. B. Sears, S. J. Chakravorty and R. D. Bendale, Phys. Rev. A 32 (1985) 2602.

[97] M. Hô, R. P. Sagar, V. H. Smith Jr. and R. O. Esquivel, J. Phys. B: At. Mol. Opt. Phys. 27 (1994) 5149.

[98] E. Jaynes, Papers on Probability Statistics and Statistical Physics, Dordrecht: Reidel, Ed. Rosencrantz.

[99] I. Bialynicki-Birula, J. Mycielski, Comm. Math. Phys. 44 (1975) 129.

[100] E. Romera and J. S. Dehesa, J. Chem. Phys. 120 (2004) 8906.

[101] J. Griffiths, Introduction to Quantum Mechanics, Prentice Hall (1995).

[102] S. Saxon, Elementos de Mecánica Cuántica, Ed. Easo (1968).

[103] Arfken, Mathematical Methods for Physicists, $3^{\text {rd }}$ Ed., Academic Press Inc. (1985).

[104] M. T. Yamashita, Revista Brasileira de Ensino de Fisica 30 (2008) n. 3. 3312.

[105] A. S. Davydov, Quantum Mechanics, Pergamon Press (1965).

[106] B. H. Bransden and C. J. Joachain, Quantum Mechanics, $2^{\text {nd }}$ Ed., England: Pearson Prentice Hall (2000), pp. 347-349.

[107] R. A. Buckingham, Proc. Roy. Soc. A 160 (1937) 94.

[108] F. M. Fernández and E. A. Castro, J. Chem. Phys. 75 (1981) 2908.

[109] S. C. Chapra and R. P. Canale, Numerical Methods for Engineers, $5^{\text {th }}$ Ed., McGraw Hill (2006) Chapter 23.

[110] M. A. Ratner and G. C. Schatz, Introduction to Quantum Mechanics in Chemistry, Prentice Hall (2001).

[111] F. A. Duarte, N. Aquino y R. A. Rojas, sin publicar

[112] J. L. Marín, R. Aceves, R. A. Rosas and S. Grijalva, Rev. Mex. Fis. E 54 (2008) 25.

[113] B. R. Frieden and M. Petri, Phys. Rev. E 86 (2012) 032102. 


\section{Apéndices}

\section{Apéndice A}

Código en Mathematica para calcular la energía del estado base del AHC dentro de una barrera esférica de paredes penetrables, usando el método variacional de Rayleigh-Ritz

Función de onda dentro de la esfera

$\psi_{i}=\operatorname{Exp}[-\alpha \mathrm{r}] *$ SphericalBessel $\left[0,\left(\gamma^{*} \mathrm{Pi} * \mathrm{r}\right) / \mathrm{R}\right]$

Se define el parámetro beta

$\beta=-1 / \mathrm{R}+\boldsymbol{\alpha}+\left(\gamma^{*} \mathrm{Pi} *\right.$ SphericalBessel $\left.]\left[1, \gamma^{*} \mathrm{Pi}\right]\right) /\left(\mathrm{R} *\right.$ SphericalBessel $\left[0, \gamma^{*}\right.$ $\mathrm{Pi}]$ )

Función de onda fuera de la esfera

$\psi_{e}=\operatorname{Exp}[(\beta-\alpha) * R] * R^{*}$ SphericalBessel] $[0, \gamma * \mathrm{Pi}] * \operatorname{Exp}[-\beta \mathrm{r}] / \mathrm{r}$

Integrales de energía cinética

cineti $=-1 / 2$ Integrate $\left[\psi_{i} \mathrm{D}\left[\mathrm{r}^{\wedge} 2 \mathrm{D}\left[\psi_{i}, \mathrm{r}\right], \mathrm{r}\right],\{\mathrm{r}, 0, \mathrm{R}\}\right]$

cinete $=-\left(\left(\beta^{*}\left(\operatorname{Exp}[(\beta-\alpha) * \mathrm{R}]{ }^{*} \mathrm{R}^{*} \text { SphericalBesselJ }\left[0, \gamma^{*} \mathrm{Pi}\right]\right)^{\wedge} 2\right) / 4\right)^{*} \operatorname{Exp}$ $[-2 * \beta * R]$

Integrales de normalización

nori $=$ Integrate $\left[\psi_{i}^{\wedge} 2 \mathrm{r}^{\wedge} 2,\{\mathrm{r}, 0, \mathrm{R}\}\right]$

nore $=\left(\operatorname{Exp}[(\boldsymbol{\beta}-\alpha) * \mathrm{R}] * \mathrm{R} * \text { SphericalBesselJ }\left[0, \gamma^{*} \mathrm{Pi}\right]\right)^{\wedge} 2 /(2 * \boldsymbol{\beta}) * \operatorname{Exp}[-2 *$ $\boldsymbol{\beta} * \mathrm{R}]$

Integrales de energía potencial 
poti $=-\mathrm{Z}$ Integrate $\left[\boldsymbol{\psi}_{i}^{\wedge} 2 \mathrm{r},\{\mathrm{r}, 0, \mathrm{R}\}\right]$

pote $=\mathrm{V} 0$ nore

Altura de la barrera, número atómico y radio de confinamiento

$\mathrm{V} 0=0 ;$

$\mathrm{Z}=2$;

$R=7 / 10 ；$

Hamiltoniano

ham $=$ cineti + cinete + poti + pote

Constante de normalización

nor $=$ nori + nore

Funcional de la energía

ener $=$ ham $/$ nor

Se encuentra el mínimo de la energía respecto a los parámetros alfa y gamma.

FindMinimum [ ener , $\{\{\alpha, 9207 / 10000\},\{\gamma, 4028 / 10000\}\}$, WorkingPrecision $\rightarrow$ 20 , AccuracyGoal $\rightarrow 6]$

$\{-1.22952005892030212416,\{\alpha \rightarrow 1.7717297500709278646, \gamma \rightarrow$ $0.46356695437731672544\}\}$ 


\section{Apéndice B}

Código en Mathematica para calcular en forma exacta las energías del AHC dentro de una barrera esférica de paredes penetrables

Radio de confinamiento

$\mathrm{rc}:=2$

Momento angular

$1:=0$

Cambios de variable

beta $:=1 /$ Sqrt $[-2 * e]$

$\mathrm{x}:=(2 * \mathrm{rc}) /$ beta

Se define la función hipergeométrica confluente dentro de la esfera con los parámetros anteriores

$\mathrm{F}:=$ Hypergeometric1F1 $[-$ beta $+1+1,2 * 1+2, \mathrm{x}]$

Derivada de F

$\mathrm{dF}:=1 / 2(1-$ beta $)$ Hypergeometric1F1 [2 - beta , $3, \mathrm{x}$ ]

Altura de la barrera

$\mathrm{V} 0:=5$

Se definen los parámetros de la solución fuera de la esfera

$\mathrm{k}:=\operatorname{Sqrt}[2 *(\mathrm{~V} 0-\mathrm{e})]$

$\mathrm{y}:=\mathrm{k}^{*} \mathrm{rc}$

Función hipergeométrica confluente fuera de la esfera

$\mathrm{G}:=$ Hypergeometric1F1 $[-1,-2 * 1,2 * \mathrm{y}]$ 
Derivada de G

$\mathrm{dG}:=0$

Función obtenida de la condición de continuidad de la derivada logarítmica

$\mathrm{H}:=2 / \operatorname{beta}^{*}(-(1 / 2)+\mathrm{l} / \mathrm{x}+\mathrm{dF} / \mathrm{F})-\mathrm{k} *(-((\mathrm{l}+1) / \mathrm{y})-1+\mathrm{dG} / \mathrm{G})$

Se encuentran las raíces de la función $\mathrm{H}$ con los parámetros dados. La raíz obtenida depende del punto de inicio de la iteración

FindRoot $[\operatorname{Re}[\mathrm{H}],\{\mathrm{e},-24 / 100\}$, WorkingPrecision $\rightarrow 35$, AccuracyGoal $\rightarrow 30$ ] $\{\mathrm{e} \rightarrow-0.27395416264426523400801024501056895\}$ 


\section{Apéndice C}

Trabajos publicados 


\section{Fine structure in the hydrogen atom boxed in a spherical impenetrable cavity}

\section{R. A. Rojas ${ }^{1}$ | N. Aquino ${ }^{1}$ (1) | A. Flores-Riveros ${ }^{2}$}

\author{
${ }^{1}$ Departamento de Física, Universidad \\ Autónoma Metropolitana-Iztapalapa, \\ Avenida San Rafael Atlixco 186, Col. \\ Vicentina, Iztapalapa, México D. F 09340, \\ México \\ ${ }^{2}$ Instituto de Física, Benemérita Universidad \\ Autónoma de Puebla, Apartado Postal J-48, \\ 72570, Puebla, Pue, México \\ Correspondence \\ N. Aquino, Departamento de Física, \\ Universidad Autónoma Metropolitana- \\ Iztapalapa, Avenida San Rafael Atlixco 186, \\ Col. Vicentina, Iztapalapa, México D. F. \\ 09340, México. \\ Email: naa@xanum.uam.mx
}

\begin{abstract}
The spectrum of the hydrogen atom confined in a spherical impenetrable box of radius $R_{\mathrm{c}}$ has been investigated by many authors up to date, but not at the level of relativistic corrections. It is well known that, as $R_{\mathrm{c}}$ diminishes, all energy levels and the pressure increase very rapidly, whereas the polarizability goes to zero. In this report, we have computed the relativistic corrections that underlie the fine structure of the confined hydrogen atom, as a function of $R_{\mathrm{c}}$. Such corrections correspond to relativistic kinetic energy, spin-orbit coupling and the Darwin term, which are calculated in the frame of time-independent perturbation theory, for which, use was made of the exact confined hydrogen atom wave functions. We show that for a confinement radius of 0.5 au the relativistic corrections increase up to three orders of magnitude with respect to those corresponding to the free atom. As $R_{\mathrm{c}}$ decreases, the kinetic energy correction and the spin-orbit coupling for $j=1 / 2$ become negative whereas their absolute value and the Darwin term, which is positive, increase very rapidly.
\end{abstract}

\section{KEYWORDS}

confined hydrogen atom, Darwin term, fine structure, kinetic energy correction, spin-orbit coupling

\section{1 | INTRODUCTION}

Eight decades have elapsed since Michels et al. ${ }^{[1]}$ proposed the first model of the confined hydrogen atom (CHA) to understand how the polarizability of the atom changes when subjected to external pressures. The model consists of a nucleus clamped at the center of an impenetrable spherical cavity of radius $R_{\mathrm{c}}$ and an electron moving around it and subject to the electrostatic attraction. Because of impenetrability a Dirichlet boundary condition is imposed on the wave function at the sphere surface. Since 1937, such a confinement model has often been utilized to simulate spatial compression on various quantum systems. ${ }^{[1-45]}$

The observable properties of a physical system change, sometimes in a drastic way, when it is spatially restricted, either within a natural cavity, as in a porous medium, or artificially through the use of electric or magnetic fields. The spatial confinement imposes additional boundary conditions on the wave functions which leads to abrupt variations on the observables of the system.

By using the model of spatial confinement within cavities of various sizes and shapes, the following systems have been studied: the electronic structure of atoms and molecules subject to high external pressures, in particular, the hydrogen and helium atoms, and the hydrogen molecular ion. Atoms with many electrons trapped inside fullerenes and zeolites; ${ }^{[42-46]}$ wires, points, and quantum wells; ${ }^{[35,47,48]}$ the specific heat of a monocrystalline solid subjected to high pressures, ${ }^{[36]}$ the transition probabilities and, the Shannon and Fisher entropies of the $\mathrm{CHA},{ }^{[37-39]}$ among others. A complete list of applications can be found in reviews and books on this topic.

When the radius of confinement decreases the electron moves around the nucleus in a reduced space thus increasing the corresponding kinetic energy, in full agreement with the Heisenberg Uncertainty Principle. Therefore, the relativistic correction to the energy of the confined atom would expectedly be greater than that of the free atom. In this work, we analyze the relativistic corrections to the hydrogen atom confined within an impenetrable sphere. The relativistic corrections are made up of three contributions: the relativistic correction to the electron kinetic energy, the spin-orbit coupling correction and the one due to the Darwin term.

This work is organized as follows: in Section 2 we give a brief description of the solution to the problem of the hydrogen atom confined in a spherical impenetrable cavity. In Section 3, we present the corrections that give rise to the CHA fine structure, taking into account fulfillment of 
Dirichlet boundary conditions for the atomic orbitals at a finite value of the radial coordinate. In Section 4, we show our results for the fine structure corresponding to the three lowest order ( $n=1,2$ and 3) shells. Finally, in Section 5 we give our conclusions.

\section{2 | EXACT SOLUTION}

In this section, we follow closely the approach used in refs. [2,11,12]. The time-independent Schrödinger equation for the $\mathrm{CHA}$ is

$$
-\frac{1}{2} \nabla^{2} \psi+V(r) \psi=E \psi
$$

where

$$
V(r)=\left\{\begin{array}{ccc}
-1 / r & \text { if } & r<R_{\mathrm{c}} \\
+\infty & \text { if } & r>R_{\mathrm{c}}
\end{array} .\right.
$$

Here $R_{\mathrm{c}}$ is the radius of the spherical box. As it is well known the wave function can be separated as the product of a radial function times the angular-dependent spherical harmonics,

$$
\psi(r, \theta, \varphi)=R(r) Y_{\operatorname{lm}}(\theta, \varphi) .
$$

By substituting Equations 3 into 1, the obtained radial Schrödinger equation is of the form

$$
-\frac{1}{2} \frac{1}{r^{2}} \frac{d}{d r}\left(r^{2} \frac{d R}{d r}\right)+\left(V(r)+\frac{I(I+1)}{2 r^{2}}\right) R=E R
$$

For the region of interest $0<r<R_{c}$, the differential Equation 4 becomes

$$
-\frac{1}{2} \frac{1}{r^{2}} \frac{d}{d r}\left(r^{2} \frac{d R}{d r}\right)+\left(\frac{I(I+1)}{2 r^{2}}-\frac{1}{r}\right) R=E R
$$

with the Dirichlet boundary conditions

$$
R\left(R_{\mathrm{c}}\right)=0
$$

With the substitutions

$$
n=1 / \sqrt{-2 E}, \quad \rho=2 r / n
$$

and by using the ansatz

$$
R=\mathrm{e}^{-\rho / 2} \rho^{\prime} F(\rho),
$$

the radial Schrödinger Equation 5 becomes

$$
\rho \frac{d^{2} F}{d \rho^{2}}+(2 I+2-\rho) \frac{d F}{d \rho}+(n+I+1) F=0
$$

Since the wave function must be finite at the origin, the solution $F$ is given by the confluent hypergeometric function.

Since the hydrogen atom is enclosed in an impenetrable sphere of radius $R_{\mathrm{c}}$, the boundary (Dirichlet) conditions require that $F=0$ at $r=R_{\mathrm{c}}$. The allowed energies are thus found when

$$
{ }_{1} F_{1}\left[-n+l+1,2 I+2, \rho_{0}\right]=0,
$$

where $\rho_{0}=2 R_{\mathrm{c}} / n$.

For a given value of $I$ the first root corresponds to the energy of the lowest state and the successively higher roots to the next excited states. In this work, the allowed energies were calculated by means of Mathematica to evaluate the hypergeometric function and following the root-finding procedure FindRoot implemented therein, which yields an accuracy of 25 significant figures. Our results are in complete agreement with those of ref. 11 up to the same number of figures. The energy eigenvalues $E_{n l}$ are labeled with the principal quantum number $n$ and the quantum angular momentum $I$.

It should be noted that the presence of impenetrable walls partially breaks the state degeneracy of the hydrogen atom. In a given shell the states with highest value of angular momentum I remain lowest in energy. The degeneracy of an energy level $E_{n l}$ is $2 l+1$ that corresponds to the possible values of the quantum number $m$.

\section{3 | FINE STRUCTURE OF THE CHA}

The Hamiltonian of the $\mathrm{CHA}$ with relativistic effects can be written as

$$
H=H^{0}+H^{\prime},
$$


TABLE 1 Kinetic energy $E_{\mathrm{r}}$ and Darwin term $E_{\mathrm{D}}$ corrections (Hartrees) for the $1 \mathrm{~s}$ CHA state as a function of $R_{\mathrm{C}}$ (Bohrs)

\begin{tabular}{|c|c|c|}
\hline$R_{\mathrm{c}}$ & $E_{\mathrm{r}}$ & $E_{D}$ \\
\hline 0.5 & -0.010890946296549 & 0.018387798215526 \\
\hline 0.6 & -0.005375519223353 & 0.011376239563636 \\
\hline 0.7 & -0.002984746976474 & 0.007660486100202 \\
\hline 0.8 & -0.001809109908346 & 0.005488572563678 \\
\hline 0.9 & -0.001173972429158 & 0.004123415229507 \\
\hline 1.0 & -0.000804830730881 & 0.003215963399520 \\
\hline 1.5 & -0.000213117125238 & 0.001338239697736 \\
\hline 2.0 & -0.000099508667876 & 0.000794323252781 \\
\hline 2.5 & -0.000063416196423 & 0.000571754335933 \\
\hline 3.0 & -0.000048435737783 & 0.000463335235954 \\
\hline 4.0 & -0.000037491951438 & 0.000373458243015 \\
\hline 5.0 & -0.000034420573658 & 0.000345560533594 \\
\hline 7.0 & -0.000033345380894 & 0.000335226817771 \\
\hline 9.0 & -0.000033284694084 & 0.000334615073264 \\
\hline 10.0 & -0.000033282585428 & 0.000334593252914 \\
\hline$\infty$ & -0.000033282096549 & 0.000026625677239 \\
\hline
\end{tabular}

where $H^{0}$ is the unperturbed Hamiltonian associated with Equation 1 and the perturbation $H^{\prime}$ is given by refs. [46, 49-51].

$$
H^{\prime}=H_{1}^{\prime}+H_{2}^{\prime}+H_{3}^{\prime}
$$

where

$$
H_{1}^{\prime}=-\frac{\left(-1 / 2 \nabla^{2}\right)^{2}}{2 c^{2}}
$$

\begin{tabular}{|c|c|c|}
\hline$R_{\mathrm{c}}$ & $E_{\mathrm{r}}$ & $E_{D}$ \\
\hline 0.5 & -0.167298589680307 & 0.064252897971641 \\
\hline 0.6 & -0.080973172259490 & 0.038641831879767 \\
\hline 0.7 & -0.043898664542648 & 0.025282380622140 \\
\hline 0.8 & -0.025865105706487 & 0.017592538314714 \\
\hline 0.9 & -0.016243417934079 & 0.012830298222922 \\
\hline 1.0 & -0.010729298009307 & 0.009709637933029 \\
\hline 1.5 & -0.002219640464883 & 0.003451275977578 \\
\hline 2.0 & -0.000751306215374 & 0.001729774648049 \\
\hline 2.5 & -0.000335761443790 & 0.001039816325285 \\
\hline 3.0 & -0.000179528158724 & 0.000696862367209 \\
\hline 4.0 & -0.000071790211447 & 0.000376521961505 \\
\hline 5.0 & -0.000037422903885 & 0.000232598160100 \\
\hline 7.0 & -0.000015420788505 & 0.000111195773681 \\
\hline 9.0 & -0.000009048909386 & 0.000068463067584 \\
\hline 10.0 & -0.000007636124840 & 0.000058354547100 \\
\hline$\infty$ & -0.000005408340689 & 0.000003328209655 \\
\hline
\end{tabular}

TABLE 2 Kinetic energy $E_{\mathrm{r}}$ and Darwin term $E_{\mathrm{D}}$ corrections (Hartrees) for the $2 \mathrm{~s}$ CHA state as a function of $R_{\mathrm{c}}$ (Bohrs) 

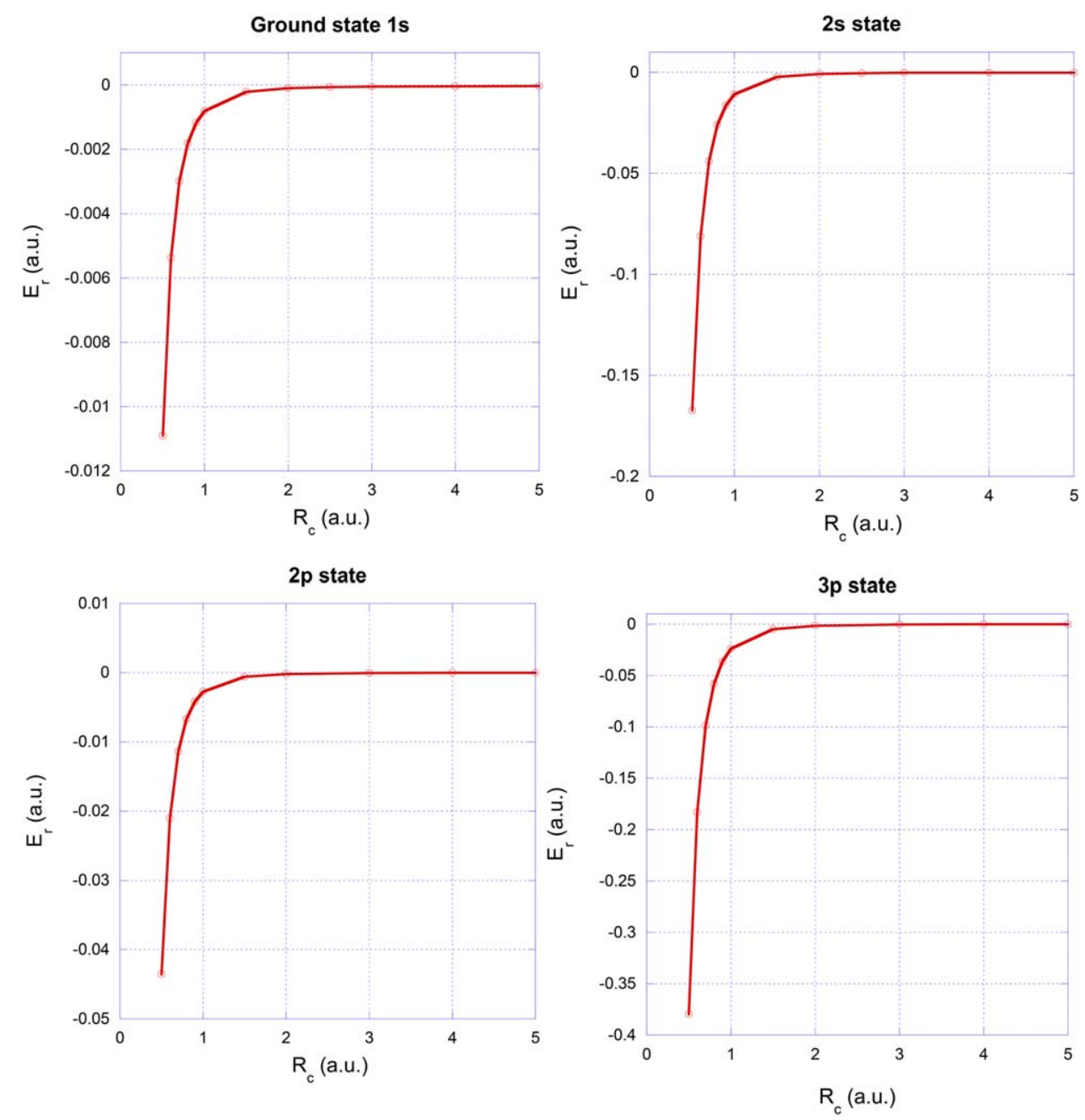

FIGURE 1 Relativistic kinetic energy corrections $E_{\mathrm{r}}$ for the $1 \mathrm{~s}, 2 \mathrm{~s}, 2 \mathrm{p}$, and $3 \mathrm{p}$ states of the CHA as a function of $R_{\mathrm{c}}$

$$
H_{2}^{\prime}=\frac{1}{2 c^{2}} \frac{1}{r} \frac{d V}{d r} \mathbf{L} \cdot \mathbf{S}
$$

and

$$
H_{3}^{\prime}=\frac{\pi}{2 c^{2}} \delta(\mathbf{r})
$$

The first term, $H_{1}^{\prime}$ is due to the kinetic energy relativistic correction, the second corresponds to the spin-orbit coupling and the third denotes the Darwin term.

The zeroth-order wave functions $\psi_{n \mid m_{l} m_{s}}^{0}$ are spin-orbitals constructed as the product of a spatial orbital $\psi_{n l m_{l}}^{0}$ (Equation 3) and a spinor $\chi_{1 / 2, m_{s}}$, with $m_{s}=1 / 2$ or $-1 / 2$

$$
\psi_{n \mid m_{l} m_{\mathrm{s}}}^{0}=\psi_{n \mid m_{l}}^{0} \chi_{\frac{1}{2}, m_{\mathrm{s}}} .
$$

The orbitals $\psi_{n l m l}^{0}$ fulfill the Schrödinger Equation 1 with energy eigenvalues $E_{n l}^{0}$

$$
H^{0} \psi_{n \mid m_{l}}^{0}=E_{n l}^{0} \psi_{n \mid m_{l}}^{0} .
$$

The unperturbed energy eigenvalues $E_{n l}^{0}$ are obtained as described in the previous section.

The spin-orbitals are simultaneous eigenfunctions of $H^{0}, L^{2} L_{z}, S^{2}$ and $S_{z}$ with eigenvalues $E_{n l}^{O}, I(I+1), m_{l}, 3 / 4$ and $m_{s}$, respectively. For each $C H A$ energy level $E_{n l}^{0}$ there are $2(2 l+1)$ spin-orbitals, where $(2 l+1)$ corresponds to the central field degeneracy and factor 2 accounts for the two spin values of $m_{\mathrm{s}}$. The CHA degeneracy is lower than that of the free hydrogen atom, which is recovered as $R_{\mathrm{c}} \rightarrow \infty$

Calculation of the first-order energy correction in perturbation theory is straightforward.

The CHA relativistic energy correction is given by 

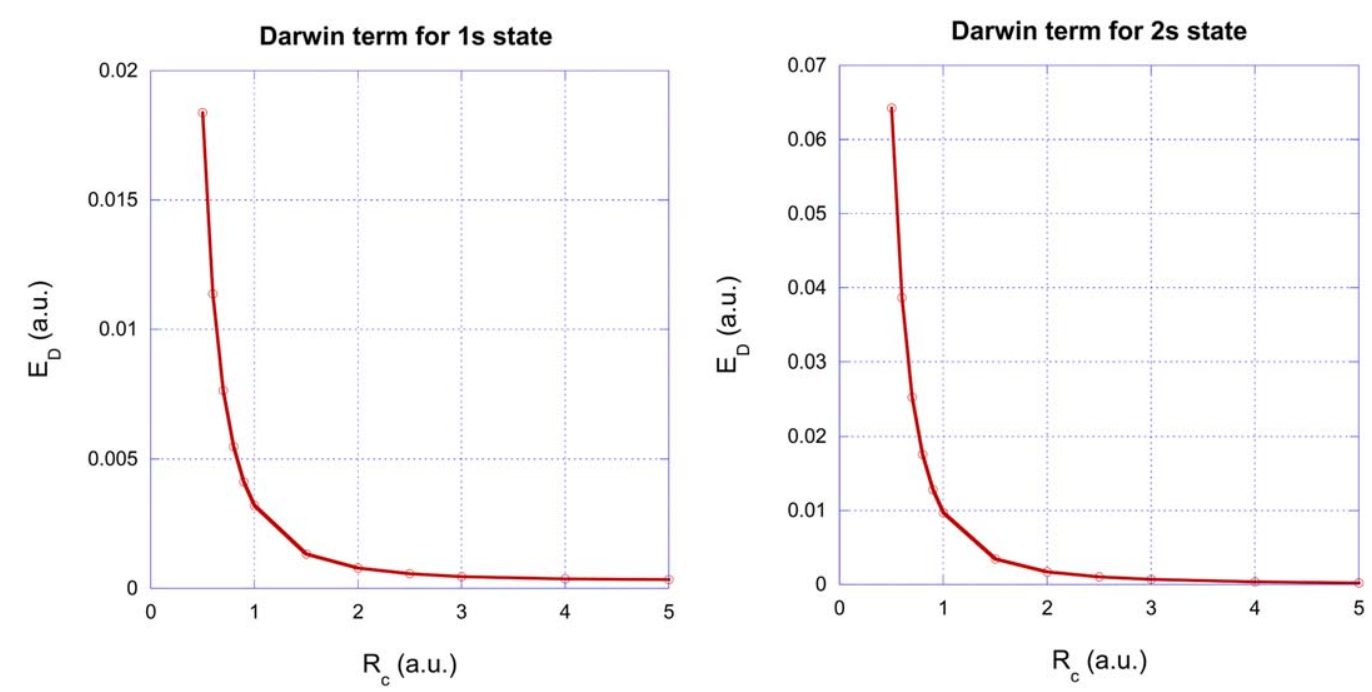

FIGURE 2 The Darwin term $E_{\mathrm{D}}$ corrections for the $1 \mathrm{~s}$ and $2 \mathrm{~s}$ CHA states as a function of $R_{\mathrm{c}}$

$$
E_{\mathrm{r}}^{1}=-\frac{1}{2 c^{2}}\left[E_{n l}^{2}+2 E_{n l}\left\langle\frac{1}{r}\right\rangle_{n l}+\left\langle\frac{1}{r^{2}}\right\rangle_{n l}\right]
$$

where the expectation values $\langle 1 / r\rangle_{n l}$ and $\left\langle 1 / r^{2}\right\rangle_{n l}$ are calculated in terms of the CHA wave functions, given by Equations 3 and 8 .

The spin-orbit correction corresponds to

$$
E_{\mathrm{so}}^{1}=\frac{[j(j+1)-l(I+1)-3 / 4]}{4 c^{2}}\left\langle\frac{1}{r^{3}}\right\rangle_{n l}
$$

where the expectation value $\left\langle 1 / r^{3}\right\rangle_{n l}$ is calculated through the $\mathrm{CHA}$ wave functions, $E_{n l}$ are the energy eigenvalue of the $\mathrm{CHA}$ states and $c=137.03599911$.

The Darwin term affects only the $S$-symmetry $(I=0)$ states because they are the only nonzero ones at the origin.

$$
E_{D}^{1}=\frac{\pi}{2 c^{2}}\left|\psi_{n 00}^{0}(0)\right|^{2}
$$

TABLE 3 Kinetic energy $E_{\mathrm{r}}$ and spin-orbit coupling $E_{\mathrm{so}}$ corrections (Hartrees) for the $2 \mathrm{p}$ CHA state as a function of $R_{\mathrm{c}}$ (Bohrs)

\begin{tabular}{llll}
\hline$R_{\mathrm{c}}$ & $E_{\mathrm{r}}$ & $E_{\mathrm{so}}(\mathbf{j}=\mathbf{1} / \mathbf{2})$ & $E_{\mathrm{so}}(\mathbf{j}=\mathbf{3} / \mathbf{2})$ \\
\hline 0.5 & -0.043517169403930 & -0.002265272040865 & 0.001132636020433 \\
\hline 0.6 & -0.021008199227508 & -0.001325069125125 & 0.000662534562563 \\
\hline .7 & -0.011353910155357 & -0.000843544280752 & 0.000421772140376 \\
\hline 0.8 & -0.006665222920648 & -0.000571333919743 & 0.000285666959872 \\
\hline 0.9 & -0.004168093747417 & -0.000405731831501 & 0.000202865915751 \\
\hline 1.0 & -0.002739926750033 & -0.000299104091074 & 0.000149552045537 \\
\hline 1.5 & -0.000548408560341 & -0.000093881178309 & 0.000046940589154 \\
\hline 2.0 & -0.000176991724021 & -0.000042080552352 & 0.000021040276176 \\
\hline 3.0 & -0.000037218817367 & -0.000014206835621 & 0.000007103417810 \\
\hline 4.0 & -0.000012985175640 & -0.000006918569911 & 0.000003459284956 \\
\hline 5.0 & -0.000006096735194 & -0.000004144104146 & 0.000002072052073 \\
\hline 7.0 & -0.000002339923559 & -0.000002149539183 & 0.000001074769591 \\
\hline 9.0 & -0.000001424244382 & -0.000001504323362 & 0.000000752161681 \\
\hline 10.0 & -0.000001237001261 & -0.000001352018201 & 0.000000676009100 \\
\hline$\infty$ & -0.000000970727816 & -0.000001109403218 & 0.000000554701609 \\
\hline & & & \\
\hline
\end{tabular}



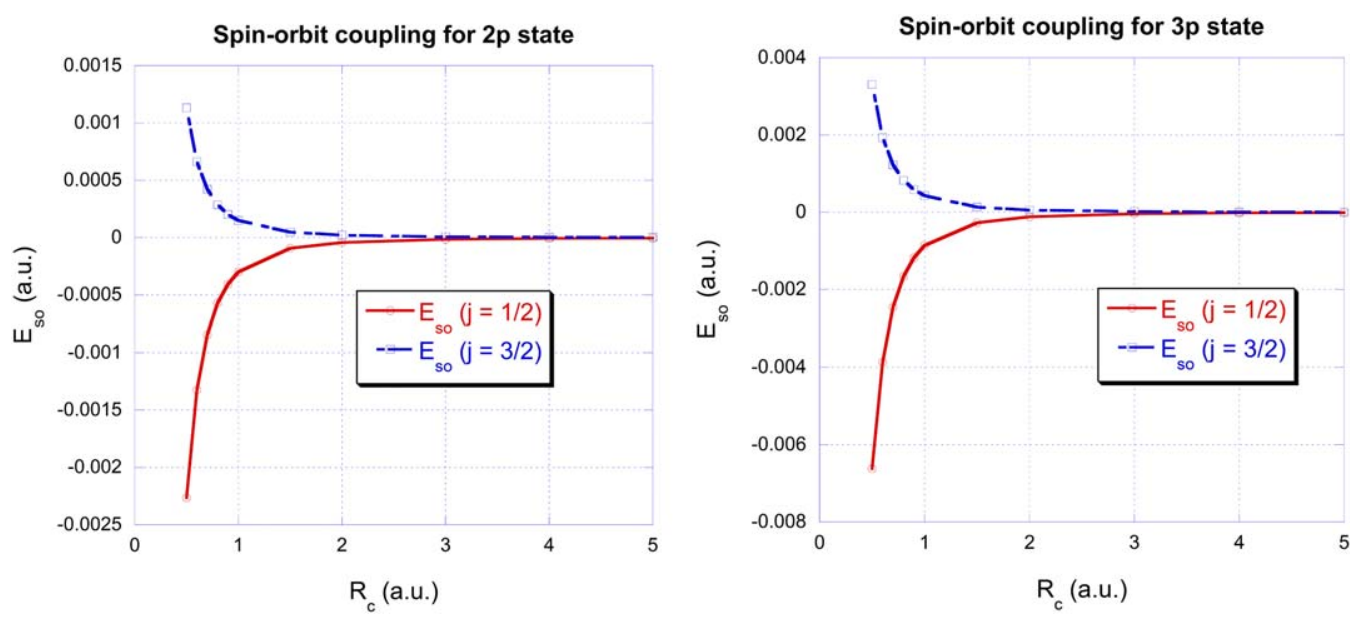

FIGURE 3 Spin-orbit coupling $E_{\mathrm{so}}$ correction for the $2 p$ and $3 p$ CHA states as a function of $R_{c}$

This term is related to the rapid motion (oscillations) of the electron around the nucleus (zitterbewegung). As the box radius diminishes, the electron kinetic energy increases and the density around the nucleus also grows, therefore we expect an increment in the Darwin term value.

The CHA energies considering the relativistic and spin-orbit corrections are

$$
E_{n j}=E_{n l}+E_{r}^{1}+E_{\text {so }}^{1}+E_{D}^{1}
$$

\section{RESULTS}

The relativistic correction to the electron kinetic energy is small compared to its rest mass energy $m c^{2}=0.511$ Mev. For example, the ratio for the CHA 3s state at $R_{\mathrm{c}}=.5 \mathrm{au}$, is approximately $9.46 \times 10^{-3} \sim \alpha$. Equation 18 thus gives the kinetic energy correction appropriately for box radii $R_{\mathrm{c}} \geq 0.5$ au.

The S-symmetry CHA states have only two contributions: the kinetic energy and the Darwin term corrections. The former becomes more negative as $R_{\mathrm{c}}$ approaches zero, whereas the latter, the Darwin term gets increasingly positive, as given in Tables 1 and 2 and Figures 1 and 2 .

TABLE 4 Kinetic energy $E_{\mathrm{r}}$ and spin-orbit coupling $E_{\mathrm{so}}$ corrections (Hartrees) for the $3 p$ CHA state as a function of $R_{\mathrm{c}}$ (Bohrs)

\begin{tabular}{llll}
\hline$R_{\mathrm{c}}$ & $E_{\mathrm{r}}$ & $E_{\mathrm{so}}(\mathbf{j}=\mathbf{1} / \mathbf{2})$ & $E_{\mathrm{so}}(\mathbf{j}=\mathbf{3} / \mathbf{2})$ \\
\hline 0.5 & -0.379614356559985 & -0.006622832029955 & 0.003311416014978 \\
\hline 0.6 & -0.183132820296056 & -0.003864656625314 & 0.001932328312657 \\
\hline 0.7 & -0.098890836199988 & -0.002454111285895 & 0.001227055642947 \\
\hline 0.8 & -0.057995490624988 & -0.001657886403586 & 0.000828943201793 \\
\hline 0.9 & -0.036225926404992 & -0.001174210759387 & 0.000587105379693 \\
\hline 1.0 & -0.023782349418593 & -0.000863246389890 & 0.000431623194945 \\
\hline 1.5 & -0.004717147734461 & -0.000266898332410 & 0.000133449166205 \\
\hline 2.0 & -0.001501509065069 & -0.000117569586662 & 0.000058784793331 \\
\hline 3.0 & -0.000302010496151 & -0.000038043281151 & 0.000019021640575 \\
\hline 4.0 & -0.000098167187962 & -0.000017556868593 & 0.000008778434296 \\
\hline 5.0 & -0.000041698731587 & -0.000009841784678 & 0.000004920892339 \\
\hline 7.0 & -0.000012012094837 & -0.000004292526224 & 0.000002146263112 \\
\hline 9.0 & -0.000005030752490 & -0.000002392911366 & 0.000001196455683 \\
\hline 10.0 & -0.000003564117674 & -0.000001886378070 & 0.000000943189035 \\
\hline$\infty$ & -0.000000410890081 & -0.000000328712065 & 0.000000164356032 \\
\hline & & & \\
\hline
\end{tabular}


TABLE 5 Kinetic energy $E_{\mathrm{r}}$ and spin-orbit coupling $E_{\mathrm{so}}$ corrections (Hartrees) for the $3 \mathrm{~d}$ CHA state as a function of $R_{\mathrm{c}}$ (Bohrs)

\begin{tabular}{|c|c|c|c|c|}
\hline$R_{\mathrm{c}}$ & $E_{r}$ & $E_{s o}(j=1 / 2)$ & $E_{s o}(j=3 / 2)$ & $E_{s o}(j=5 / 2)$ \\
\hline 0.5 & -0.117564097569745 & -0.003605091194179 & -0.001802545597089 & 0.001201697064726 \\
\hline 0.6 & -0.056706232917823 & -0.002094309431948 & -0.001047154715974 & 0.000698103143983 \\
\hline 0.7 & -0.030615417553003 & -0.001323973105667 & -0.000661986552833 & 0.000441324368556 \\
\hline 0.8 & -0.017950835348891 & -0.000890414939098 & -0.000445207469549 & 0.000296804979699 \\
\hline 0.9 & -0.011209927193594 & -0.000627819063718 & -0.000313909531859 & 0.000209273021239 \\
\hline 1.0 & -0.007357279283167 & -0.000459485964342 & -0.000229742982171 & 0.000153161988114 \\
\hline 1.5 & -0.001456551256460 & -0.000138902920606 & -0.000069451460303 & 0.000046300973535 \\
\hline 2.0 & -0.000462366927413 & -0.000059825371435 & -0.000029912685717 & 0.000019941790478 \\
\hline 2.5 & -0.000190211994013 & -0.000031292022908 & -0.000015646011454 & 0.000010430674303 \\
\hline 3.0 & -0.000092238821654 & -0.000018512590938 & -0.000009256295469 & 0.000006170863646 \\
\hline 4.0 & -0.000029623333499 & -0.000008179898627 & -0.000004089949313 & 0.000002726632876 \\
\hline 5.0 & -0.000012386627300 & -0.000004399960086 & -0.000002199980043 & 0.000001466653362 \\
\hline 7.0 & -0.000003428293660 & -0.000001787758862 & -0.000000893879431 & 0.000000595919621 \\
\hline 9.0 & -0.000001376736989 & -0.000000951897935 & -0.000000475948968 & 0.000000317299312 \\
\hline 10.0 & -0.000000959043756 & -0.000000742709011 & -0.000000371354506 & 0.000000247569670 \\
\hline$\infty$ & -0.000000147920429 & -0.000000197227239 & -0.000000098613619 & 0.000000065742413 \\
\hline
\end{tabular}

The states associated with $I>0$ have only the kinetic energy and spin-orbit coupling corrections. For the free hydrogen atom the $2 s_{1 / 2}$ and 2 $p_{1 / 2}$ levels possess the same energy. In Dirac's theory the two states with the quantum numbers $(n, j)$ such that $j=l \pm 1 / 2$, have the same energy. This degeneracy is removed when quantum electrodynamics effects are considered.

However, for the CHA the degeneracy of states in the same shell with different value of $I$ is partially removed by the repulsive infinite wall. For example, the CHA states $2 \mathrm{~s}$ and $2 \mathrm{p}$ are nondegenerate for $R_{\mathrm{c}}<10$ au. The energy difference $\Delta E=E_{20}-E_{21}$ as a function of $R_{\mathrm{c}}$, is a few orders of magnitude higher than the relativistic corrections (see Tables 3 and 5). For this reason the state $2 s_{1 / 2}$ will have a different energy as compared to the two $2 p_{1 / 2}, 2 p_{3 / 2}$ states. In addition, the perturbative corrections of the states $2 p_{1 / 2}, 2 p_{3 / 2}$ have different values (see Table 3 and Figure 3 ). Therefore, these states will have different energies for boxes with $R_{\mathrm{c}}<10$ au.

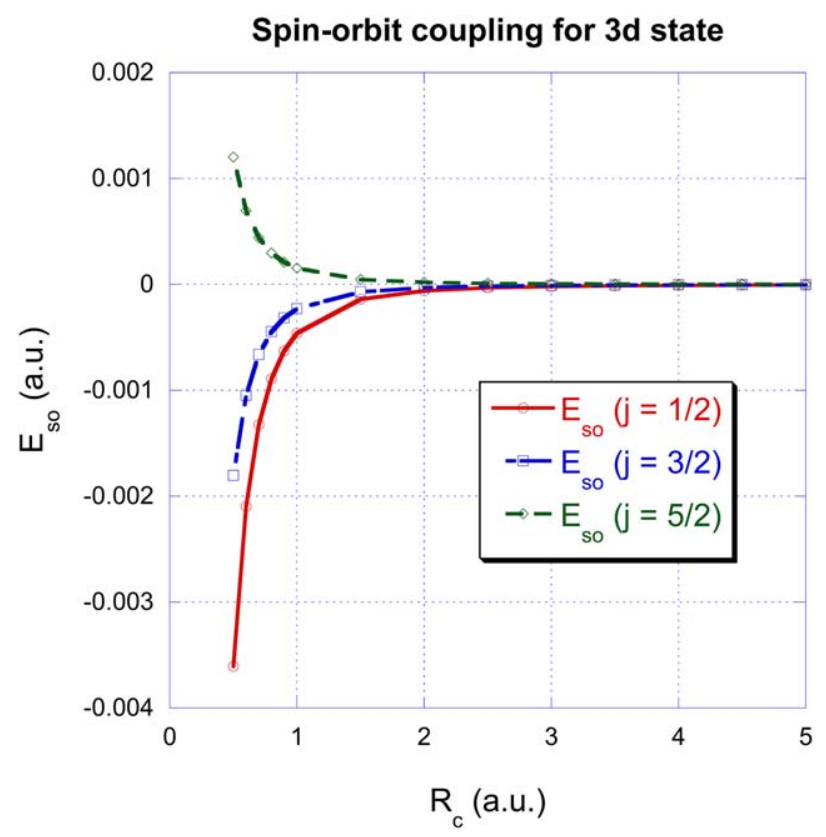

FIGURE 4 Spin-orbit coupling $E_{\mathrm{so}}$ correction for the $3 \mathrm{~d}$ CHA state as a function of $R_{\mathrm{c}}$ 
Regarding shell $n=3$, as given in Table 4, the spin-orbit coupling correction is lower for the $3 p_{1 / 2}$ state, in comparison to the $3 p_{3 / 2}$ state (see Figure 3). Whereas for the $3 d$ state, the ordering in the spin-orbit coupling correction is $E_{\mathrm{so}}\left(3 d_{1 / 2}\right) \leq E_{\mathrm{so}}\left(3 d_{3 / 2}\right) \leq E_{\mathrm{so}}\left(3 d_{5 / 2}\right)$, for all $R_{\mathrm{c}}$ values here considered (see Table 5 and Figure 4). Therefore for states with the same $n$ and I values, the one with lowest $j$ has the smallest spin-orbit coupling correction.

\section{5 | CONCLUSIONS}

We have studied the fine structure spectrum of the CHA inside hard spherical walls.

The states of a given shell are all energy degenerate for the free atom. This degeneracy is partially removed by spatial confinement, where states with lowest angular momentum I are highest in energy.

Albeit the atom kinetic energy for all sates increases under confinement of the atom, it remains within values at a non-relativistic range. However, the relativistic effects, although small, give rise to important corrections to the fine structure which should be detectable since they are a few orders of magnitude higher than those for the free hydrogen atom.

Fine structure arises due to three different mechanisms: (1) a relativistic correction for the electron kinetic energy that becomes more negative as the confinement cavity is reduced. This relativistic effect is small enough to be treated by non-relativistic quantum mechanics. (2) A spin-orbit coupling term due to the interaction between the orbital angular and spin momenta of the electron in the atom. This term splits the energy levels for a given I where the energies of these new states are determined by the principal quantum number $n$ and the total angular momentum $j$. As the atom confinement is enhanced, the gap between these states becomes larger. For states with the same $n$ and $/$ values, the one with lowest $j$ has the smallest spin-orbit coupling correction. (3) The Darwin term, which is a relativistic correction to the potential energy and acts only at the origin, has to be taken into account for the $S$-symmetry $(I=0)$ states, which are the only ones affected by such term, increasing their energy as the confinement exerted on the atom becomes stronger. In other words, the CHA fine structure becomes more important as the compression regime is increased. Nevertheless, the fine structure corrections are small in comparison to the unperturbed energy eigenvalues, so that they can be treated as small perturbations and time-independent perturbation theory holds valid.

Analysis of the effects on the $\mathrm{CHA}$ fine structure when the atom is placed inside soft spherical walls is in progress.

\section{ACKNOWLEDGMENTS}

One of us (RAR) is grateful to Universidad Autónoma Metropolitana for provision of funds through a PhD studies scholarship.

\section{ORCID}

N. Aquino (D) http://orcid.org/0000-0002-3795-0304

\section{REFERENCES}

[1] A. Michels, J. de Boer, A. Bijl, Physica 1937, 4, 981.

[2] A. Sommerfeld, H. Welker, Ann. Phys. 1938, 32, 56.

[3] S. R. de Groot, C. A. ten Seldam, Physica 1946, XII, 669.

[4] R. B. Dingle, Proc. Camb. Phil. Soc. 1953, 49, 103.

[5] D. Suryanrayana, J. A. Weil, J. Chem. Phys. 1975, 64, 510.

[6] S. Goldman, C. Joslin, J. Phys. Chem. 1992, 96, 6021.

[7] B. L. Burrows, M. Cohen, Int. J. Quantum Chem. 2006, 106, 478.

[8] B. L. Burrows, M. Cohen, Phys. Rev. A 2005, 72, 032508.

[9] H. E. Montgomery, Int. J. Mol. Sci. 2001, 2, 103.

[10] N. Aquino, Int. J. Quantum Chem. 1995, 54, 107.

[11] N. Aquino, G. Campoy, H. E. Montgomery, Jr, Int. J. Quantum Chem. 2007, 107, 1548.

[12] E. Ley-Koo, S Rubinstein, J. Chem. Phys. 1979, 71, 351.

[13] E. V. Ludeña, J. Chem. Phys. 1977, 66, 468.

[14] F. M. Fernández, E. A. Castro, J. Math. Phys. 1982, 23, 1103.

[15] C. Laughlin, B. L. Burrows, M. Cohen, J. Phys. B: At. Mol. Opt. Phys. 2002, 25, 4365.

[16] W. Wilcox, Am. J. Phys. 1989, 57, 526.

[17] J. Killingbeck, Phys. Lett. 1981, 84A, 95.

[18] P. W. Fowler, Mol. Phys. 1984, 53, 865.

[19] J. L. Marín, S. A. Cruz, J. Phys. B: At. Mol. Opt. Phys. 1992, 25, 4365. 
[20] M. E. Changa, A. V. Scherbinin, V. I. Pupishev, J. Phys. B: At. Mol. Opt. Phys. 2000, 33, 421.

[21] S. Ting-Yun, Q. Hao-Xue, L. Bai-Wen, J. Phys. B: At. Mol. Opt. Phys. 2000, 33, L349.

[22] K. D. Sen, J. Garza, R. Vargas, N. Aquino, Phys. Lett. A 2002, 295, 299.

[23] E. Ley-Koo, S. A. Cruz, J. Chem. Phys. 1981, 74, 4603.

[24] V. I. Pupishev, V. V. Bobrikov, Int. J. Quantum Chem. 2004, 100, 528.

[25] T. Guillot, Planet. Space Sci. 1999, 47, 1183.

[26] A. K. Roy, Int. J. Quantum Chem. 2015, 115, 937.

[27] S. B. Doma, F. N. El-Gammal, J. Theor. Appl. Phys. 2012, 6, 28.

[28] S. A. Cruz, E. Ley-Koo, R. Cabrera-Trujillo, Phys. Rev. A 2013, 78, 032905.

[29] A. Al-Jamel, Appl. Phys. Res. 2015, 7, 80.

[30] N. Aquino, A. Flores-Riveros, J. F. Rivas-Silva, Phys. Lett. A 2003, 307, 326.

[31] N. Aquino, J. Garza, A. Flores-Riveros, J. F. Rivas-Silva, K. D. Sen, J. Chem. Phys. 2006, 124, 054311.

[32] A. Flores-Riveros, A. Rodríguez-Contreras, Phys. Lett. A 2008, 372, 6175.

[33] T. D. Young, R. Vargas, J. Garza, Phys. Lett. A 2016, 380, 712.

[34] X. Y. Pan, V. Sahni, L. Massa, K. D. Sen, Comput. Theor. Chem. 2011, 965, 202.

[35] G. Campoy, N. Aquino, V. D. Granados, J. Phys. B: At. Mol. Opt. Phys. 2001, 35, 4903.

[36] N. Aquino, V. D. Granados, H. Yee-Madeira, Rev. Mex. Fís. 2009, 55, 125.

[37] K. D. Sen, J. Chem. Phys. 2005, 123, 074110.

[38] N. Aquino, A. Flores-Riveros, J. F. Rivas-Silva, Phys. Lett. A 2013, 377, 2062.

[39] L. G. Jiao, L. R. Zan, Y. Z. Zhang, Y. K. Ho, Int. J. Quantum Chem. 2017, 117, e25375.

[40] F. M. Fernández, E. A. Castro, Kinam 1982, 4, 193.

[41] P. O. Fröman, S. Yngve, N. Fröman, J. Math. Phys. 1987, 28, 1813.

[42] W. Jaskólski, Phys. Rep. 1996, 271, 1.

[43] N. Aquino, Adv. Quantum Chem. 2009, 57, 123.

[44] A. L. Buchachenko, J. Phys. Chem. B 2001, 105, 5839.

[45] J. P. Connerade, P. Kengkan, in Proc. Idea-Finding Symposium, Frankfurt Institute for Advanced Studies 2003, pp. 35-46. https://fias.uni-frankfurt.de/ historical/symposium/papers/connerade.pdf.

[46] B. H. Bransden, C. J. Joachain, Physics of Atoms and Molecules, 2nd ed., Prentice Hall, New York 2003, Ch. 5.

[47] G. W. Bryant, Phys. Rev B 1984, 29, 6632.

[48] L. Bányai, S. W. Koch, Semiconductor Quantum Dots, World Scientific, Singapore 1993.

[49] B. H. Bransden, C. J. Joachain, Quantum Mechanics, 2nd ed., Pearson, England 2000, pp. 390-396.

[50] M. Weissbluth, Atoms and Molecules, Academic Press, New York 1978, pp. 337-345.

[51] V. Zelevinsky, Quantum Physics, Volume 1: From Basics to Symmetries and Perturbations, Wiley-VCH Verlag GmbH \& Co. KGaA, Germany 2011, Ch. 23 .

How to cite this article: Rojas RA, Aquino N, Flores-Riveros A. Fine structure in the hydrogen atom boxed in a spherical impenetrable cavity. Int J Quantum Chem. 2017;e25584. https://doi.org/10.1002/qua.25584 
DOI: $10.1002 /$ qua.25612

\title{
ERRATUM: Fine structure in the hydrogen atom boxed in a spherical impenetrable cavity
}

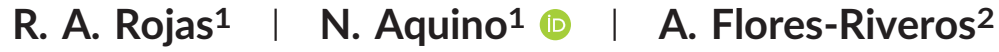 \\ ${ }^{1}$ Departamento de Física, Universidad Autónoma Metropolitana-Iztapalapa, Avenida San Rafael Atlíxco 186, Col. Vicentina, Iztapalapa, México, D. F. 09340, México \\ ${ }^{2}$ Instituto de Física, Benemérita Universidad Autónoma de Puebla, Apartado Postal J-48, 72570, Puebla, Pue, México \\ Correspondence \\ Email: naa@xanum.uam.mx
}

In the published article mentioned above, Tables 1 and 2 contain erroneous terms. The Darwin term, third columns in Tables 1, 2 and in Figure 2 , must be multiplied by a factor $\frac{1}{4 \pi}$ that correspond to $\left|Y_{00}\right|^{2}$, the angular part of the states $1 \mathrm{~s}$ and $2 \mathrm{~s}$. The correct values of the Darwing term in Tables 1, 2 and Figure 2 are the following.

TABLE 1 Kinetic energy Er and Darwin term ED corrections (Hartrees) for the 1s CHA state as a function of Rc (Bohrs)

\begin{tabular}{|c|c|c|}
\hline$R_{c}$ & $E_{r}$ & $E_{D}$ \\
\hline 0.5 & -0.010890946296549 & 0.001463254489289 \\
\hline 0.6 & -0.005375519223353 & 0.000905292380175 \\
\hline 0.7 & -0.002984746976474 & 0.000609602114667 \\
\hline 0.8 & -0.001809109908346 & 0.000436766727014 \\
\hline 0.9 & -0.001173972429158 & 0.000328130958098 \\
\hline 1.0 & -0.000804830730881 & 0.000255918235918 \\
\hline 1.5 & -0.000213117125238 & 0.000106493731468 \\
\hline 2.0 & -0.000099508667876 & 0.000063210236046 \\
\hline 2.5 & -0.000063416196423 & 0.000045498764399 \\
\hline 3.0 & -0.000048435737783 & 0.000036871046555 \\
\hline 4.0 & -0.000037491951438 & 0.000029718862707 \\
\hline 5.0 & -0.000034420573658 & 0.000027498833529 \\
\hline 7.0 & -0.000033345380894 & 0.000026676502553 \\
\hline 9.0 & -0.000033284694084 & 0.000026627821471 \\
\hline 10.0 & -0.000033282585428 & 0.000026626085063 \\
\hline$\infty$ & -0.000033282096549 & 0.000026625677239 \\
\hline
\end{tabular}




\section{WI LEY QUANTUM}

TABLE 2 Kinetic energy Er and Darwin term ED corrections (Hartrees) for the 2s CHA state as a function of Rc (Bohrs)

\begin{tabular}{|c|c|c|}
\hline$R_{c}$ & $E_{r}$ & $E_{D}$ \\
\hline 0.5 & -0.167298589680307 & 0.005113083160083 \\
\hline 0.6 & -0.080973172259490 & 0.003075019276895 \\
\hline 0.7 & -0.043898664542648 & 0.002011907924572 \\
\hline 0.8 & -0.025865105706487 & 0.001399969717160 \\
\hline 0.9 & -0.016243417934079 & 0.001021002691761 \\
\hline 1.0 & -0.010729298009307 & 0.000772668436337 \\
\hline 1.5 & -0.002219640464883 & 0.000274643815903 \\
\hline 2.0 & -0.000751306215374 & 0.000137651092836 \\
\hline 2.5 & -0.000335761443790 & 0.000082745954038 \\
\hline 3.0 & -0.000179528158724 & 0.000055454545198 \\
\hline 4.0 & -0.000071790211447 & 0.000029962665678 \\
\hline 5.0 & -0.000037422903885 & 0.000018509573467 \\
\hline 7.0 & -0.000015420788505 & 0.000008848678516 \\
\hline 9.0 & -0.000009048909386 & 0.000005448117813 \\
\hline 10.0 & -0.000007636124840 & 0.000004643707311 \\
\hline$\infty$ & -0.000005408340689 & 0.000003328209655 \\
\hline
\end{tabular}
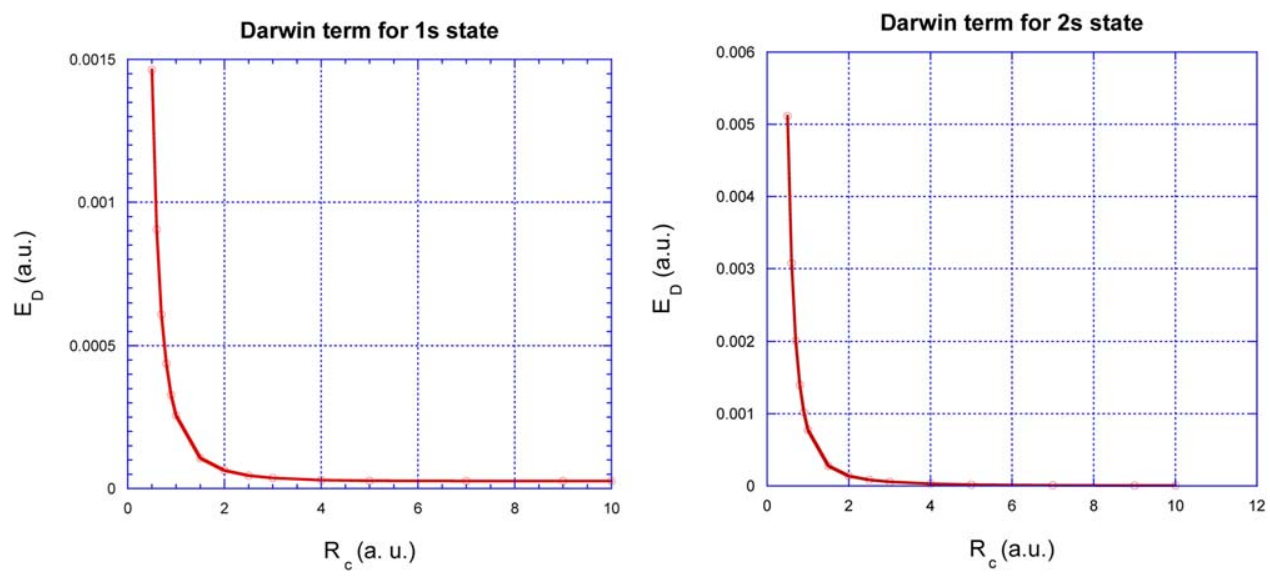

FIGURE 2 Darwin term $E_{D}$ corrections for the $1 \mathrm{~s}$ and $2 \mathrm{~s}$ CHA states as a function of $R_{c}$

The authors apologize for their hindsight and would like to thank Professor D.Baye for pointing out these errors. 


\title{
The energy correction due to a finite size nucleus of the hydrogen atom confined in a penetrable spherical cavity
}

\author{
N. Aquino ${ }^{a}$, R.A. Rojas ${ }^{a}$ and H.E. Montgomery ${ }^{b}$ \\ ${ }^{a}$ Departamento de Física, Universidad Autónoma Metropolitana-Iztapalapa, \\ Av. San Rafael Atlixco 186, Col. Vicentina, Iztapalapa, 09340 Ciudad de México. \\ ${ }^{b}$ Chemistry Program, Centre College, 600 West Walnut Street, Danville, KY 40422.
}

Received 17 January 2018; accepted 18 March 2018

\begin{abstract}
We have computed accurate values for the ground state energy of a hydrogen atom confined by a finite spherical barrier of height $V_{0}$ as a function of the confinement radius $R_{c}$. We consider the nucleus as a sphere with a uniform charge distribution instead of as a point particle. The contribution to the ground state energy due to the finite nuclear size is computed as a function of the confinement radius, $R_{c}$, and the height of the barrier, $V_{0}$, using time-independent perturbation theory. For an impenetrable cavity with $R_{c}=0.5$ au, we found that this energy correction is fifty times higher than the corresponding value for the free hydrogen atom. For a finite value of $V_{0}$, we found that the maximum of the energy correction is reached at a value $R_{\mathrm{cmax}}$, which is very close to the position at which the electron density is most compact around the nucleus. This is confirmed though evaluation of the Shannon entropy in configuration space.
\end{abstract}

Keywords: Confined hydrogen atom; finite nucleus correction; Shannon entropy.

PACS: 67.63.Gh; 67.80.fh

\section{Introduction}

Eighty years ago, Michels et al. [1] used the confined hydrogen atom (CHA) as a model to study the change in the polarizability of a hydrogen atom subjected to high external pressure. In this model, the nucleus of the hydrogen atom was clamped at the center of an impenetrable sphere of radius $R_{c}$, while the electron could move within the included volume. In this system, ionization is not possible. The way to account for ionization is to allow the walls to be penetrable. This penetrable model was successfully used by Ley-Koo and Rubinstein [2] to explain the ionization of a hydrogen atom trapped in alpha-quartz [3-4].

Many applications have been developed from these models and they have been generalized to cavities with different geometries. This model has subsequently been applied to a wide range of physical problems [1-13]. Observable properties of the systems such the energy spectrum, transition frequencies and probabilities, polarizability and the behavior of atoms trapped in fullerenes, etc., are changed by spatial confinement. Reviews and books on those topics are available [5]. Recent experimental studies show that the electron capture nuclear decay rate is increased under compression [1416]. A partial explanation of this effect was given using the model of many-electron atoms confined in an impenetrable spherical cavity [17]. With the advent of technology to construct atomic scale confinements, the study of confined systems has become increasingly relevant.

In most of the works on the properties of atoms and molecules, either free of any confinement or confined in cavities, it is assumed that the nuclei are points with charge and mass but without extent. The inclusion of a nucleus of finite size in the free hydrogen atom is accompanied by a shift in the electron energy [20-26]. The magnitude of this shift is very small compared to the energy of the hydrogen atom with a point nucleus. Until now, no one has studied how this energy shift changes for the hydrogen atom, confined in spherical penetrable cavities, when a nucleus of finite size is considered.

Pyarelal and Bhatnagar [20] proposed a model of the hydrogen atom with an impenetrable nucleus of finite size. In that model, the wave functions must vanish at the surface of the nucleus. The reduction in the volume available for movement of the electron produces an increase in the energy of the electronic states. This problem has an exact solution, but the model is unrealistic.

A more realistic model consists of a spherical nucleus of radius $r_{0}$, with an uniform distribution of charge. For hydrogen-like atoms with a small nuclear charge, $Z$, it is well-known that the Schrödinger equation adequately describes those systems [19]. An exact solution to this problem was found by Ley-Koo et al. [19], in which they studied the muonic atoms, free of any confinement, with $Z$ up to 90 . In this work, we will adopt the model of a spherical nucleus with an uniform charge distribution, and we will use firstorder perturbation theory to calculate the correction to the ground-state energy of the hydrogen atom confined in spherical penetrable and impenetrable cavities, as a function of the radius of confinement $R_{c}$.

The objectives of this work are twofold: the former is to establish benchmark values for the energy of the ground state of the hydrogen atom confined in a spherical penetrable cavity, the second is to calculate the energy shift of this system when considering a spherical nucleus of finite volume with an uniform distribution of charge.

The organization of this work is as follows: in Sec. 2, we solve CHA with a point nucleus in a spherical, padded cavity. In Sec. 3 we use first-order perturbation theory to compute 
the energy correction due to inclusion of a nucleus of finite size. In Sec. 4 we show the results of the calculation of the energy correction due to a finite size nucleus, obtained for both penetrable and impenetrable confinements. Finally, in Sec. 5 we give our conclusions.

\section{The CHA in a spherical padded cavity}

In this section we give a brief description of the solution of the hydrogen atom confined in a spherical padded cavity. A detailed explanation can be found in Refs. 2, 7 and 9.

In atomic units ( $m=e=\hbar=1$ ), the Schrödinger equation for a hydrogen atom at the center of a sphere of radius $R_{c}$ and confined by a constant potential $V_{0}$ is

$$
-\frac{1}{2} \nabla^{2}+V(r) \Psi(r, \theta, \phi)=E \Psi(r, \theta, \phi),
$$

where the potential is

$$
V_{c}=\left\{\begin{array}{cc}
-\frac{1}{r}, & 0 \leq r \leq R_{c} \\
V_{0}, & r>R_{c}
\end{array} .\right.
$$

Equation (1) can be solved using separation of variables

$$
\psi(r, \theta, \phi)=R(r) Y_{l, m}(\theta, \phi)
$$

where $Y_{l, m}(\theta, \phi)$ is a normalized spherical harmonic and $R(r)$ is a radial function composed of $R_{i}(r)$, the wavefunction inside the sphere and $R_{e}(r)$, the wavefunction external to the sphere.

The Schrödinger equation for the inner region $0 \leq r \leq$ $R_{c}$

$$
\begin{aligned}
& {\left[-\frac{1}{2}\left(\frac{1}{r^{2}} \frac{d}{d r} r^{2} \frac{d}{d r}\right)+\frac{l(l+1)}{2 r^{2}}-\frac{1}{r}\right]} \\
& \times R_{i}(r)=E R_{i}(r),
\end{aligned}
$$

whose solution is given by [8-9]:

$$
R_{i}(r)=A e^{-\rho / 2} \rho^{l} M(-\beta+l+1,2 l+2, \rho),
$$

where $M(a, b, r)$ is the confluent hypergoemetric function [18] and

$$
\beta=\frac{1}{\sqrt{-2 E}}, \quad \rho=2 r / \beta .
$$

The external region, $r>R_{c}$, is described by the Schrödinger equation

$$
\begin{aligned}
& {\left[-\frac{1}{2}\left(\frac{1}{r^{2}} \frac{d}{d r} r^{2} \frac{d}{d r}\right)+\frac{l(l+1)}{2 r^{2}}+V_{0}\right]} \\
& \times R_{e}(r)=E R_{e}(r),
\end{aligned}
$$

which can be written as

$$
\left(\frac{d^{2}}{d y^{2}}+\frac{2}{y} \frac{d}{d y}-\frac{l(l+1)}{y^{2}}-1\right) R_{e}(r)=0
$$

where

$$
y=k r, \quad k^{2}=2 \mu\left(V_{0}-E\right) / \hbar^{2},
$$

and whose solution is given by [2]

$$
R_{e}(y)=B y^{-l-1} e^{-y} M(-l,-2 l, 2 y)
$$

Hereafter, we restrict our attention to states with $l=0$ because these are the only states which have non-zero value of $R_{i}(r)$ when $r=0$.

The eigenvalues are determined by the requirement that the total wavefunction must be continuous with continuous first derivatives at $r=R_{c}$. This is most easily accomplished by matching logarithmic derivatives at $R_{c}$, resulting in the equation

$$
\left.\frac{d}{d r}\left[\ln R_{i}\right]\right|_{R_{c}}-\left.\frac{d}{d r}\left[\ln R_{e}\right]\right|_{R_{c}}=0
$$

The normalization constants $A$ and $B$ are found from the requirements that

$$
R_{i}\left(\frac{2 R_{c}}{\beta}\right)=R_{e}\left(k R_{c}\right) .
$$

and the normalization condition

$$
\int_{0}^{r_{0}}\left(R_{i}\right)^{2} r^{2} d r+\int_{r_{0}}^{\infty}\left(R_{e}\right)^{2} r^{2} d r=1 .
$$

As mentioned above, the zeroes of the logarithmic derivative equation are the eigenvalues of the problem. The first zero corresponds to the ground state energy. With this value, we construct the wave function in each region. To find the zeroes of the equation we used Mathematica 9 with the command FindRoot with 50-digit precision variables. The eigenvalues obtained through this procedure are shown in Tables I$\mathrm{V}$ as a function of the box radius $R_{c}$ and the potential height $V_{0}$. These results are in complete agreement with previous calculations $[2,7,9]$. The results are shown with 15 digits after the decimal point.

\section{Finite nucleus size correction}

The Hamiltonian of the hydrogen atom with a finite nucleus confined by a spherical penetrable wall is given by

$$
H=-\frac{\nabla^{2}}{2}+V_{r}(r)
$$

where

$$
V_{r}(r)=\left\{\begin{array}{ll}
\frac{1}{2 r_{0}}\left[\left(\frac{r}{r_{0}}\right)^{2}-3\right], & 0<r<r_{0} \\
-\frac{1}{r}, & r_{0}<r<R_{c} \\
V_{0}, & R_{c}<r<\infty
\end{array},\right.
$$


TABLE I. CHA ground state energy $E_{10}^{(1)}$, and first-order correction due to the finite nucleus, $E_{10}^{(1)}\left(R_{c}\right)$, as a function of the confinement radius $R_{c}$ for $V_{0}=0$. Also shown is the ratio between the energy correction of the confined system, $E_{10}^{(1)}\left(R_{c}\right)$, and the correction of the free hydrogen atom $E_{10}$. Energies are in hartrees and distances are in bohrs.

\begin{tabular}{cccc}
\hline$R_{c}$ & $E_{10}$ & $E_{10}^{(1)}\left(10^{-10}\right)$ & $E_{10}^{(1)}\left(R_{c}\right) / E_{10}^{(1)}$ \\
\hline 0.75 & -0.002551608753406 & 0.342781939506244 & 0.345 \\
0.8 & -0.017424391031037 & 0.808141286815622 & 0.814 \\
0.9 & -0.067406311452319 & 1.325196851197549 & 1.335 \\
1.0 & -0.125000000000000 & 1.544741614031751 & 1.556 \\
1.1 & -0.180067083549980 & 1.621160850903080 & 1.633 \\
1.2 & -0.229179151514072 & 1.627437259807232 & 1.639 \\
1.5 & -0.338167417956141 & 1.507712255705773 & 1.519 \\
2.0 & -0.431218889241793 & 1.287458459987721 & 1.160 \\
2.5 & -0.470393522970229 & 1.151246460119175 & 1.083 \\
3.0 & -0.487223082818398 & 1.075285232620169 & 1.042 \\
3.5 & -0.494519692585279 & 1.034355146835889 & 1.020 \\
4.0 & -0.497674689400819 & 1.012980250520460 & 1.009 \\
5.5 & -0.499025598864902 & 1.002187518743767 & 0.004 \\
\hline
\end{tabular}

TABLE II. CHA ground state energy $E_{10}^{(1)}$, and first-order correction due to the finite nucleus, $E_{10}^{(1)}\left(R_{c}\right)$, as a function of the confinement radius $R_{c}$ for $V_{0}=5$. Also shown is the ratio between the energy correction of the confined system, $E_{10}^{(1)}\left(R_{c}\right)$, and the correction of the free hydrogen atom $E_{10}$. Energies are in hartrees and distances are in bohrs.

\begin{tabular}{cccc}
\hline$R_{c}$ & $E_{10}$ & $E_{10}^{(1)}\left(10^{-10}\right)$ & $E_{10}^{(1)}\left(R_{c}\right) / E_{10}^{(1)}$ \\
\hline 0.4 & 4.827691517791768 & 6.456668836007435 & 6.506 \\
0.5 & 3.907609648085746 & 10.240494963124124 & 10.319 \\
0.6 & 2.982382648801240 & 9.598999388792230 & 9.672 \\
0.8 & 1.666642892127310 & 6.980485974979947 & 7.034 \\
0.9 & 1.229573168163173 & 5.926377707785826 & 5.972 \\
1.0 & 0.893377387585463 & 5.081081669583943 & 5.120 \\
1.5 & 0.028688218476904 & 2.781656875168397 & 2.803 \\
2.0 & -0.273954162644265 & 1.889596355389960 & 1.904 \\
2.5 & -0.397386813501905 & 1.473407522212738 & 1.484 \\
3.0 & -0.452163079706601 & 1.256864293020748 & 1.266 \\
3.5 & -0.477542879189045 & 1.137865941478808 & 1.146 \\
4.0 & -0.489507801753420 & 1.071253920964084 & 1.079 \\
4.5 & -0.495153894267392 & 1.034166451405600 & 1.042 \\
5.0 & -0.497794417047201 & 1.013927192430064 & 1.021 \\
\hline
\end{tabular}

The first two terms in Eq. (15) are the usual terms of a free hydrogen atom with a finite size nucleus [6,19,21-23], and $r_{0}$ is the radius of the hydrogen atom, a proton. The third term is introduced to confine the hydrogen atom in a spherical cavity of radius $R_{c}$.

We define the potential

$$
\tilde{V}(r)=\left\{\begin{array}{ll}
-\frac{1}{r}, & r<r<R_{0} \\
0, & r_{0}<r<R_{c}
\end{array} .\right.
$$

Adding and subtracting $\tilde{V}(r)$ to Eq. (14), and grouping terms we obtain the

$$
H=-\frac{\nabla^{2}}{2}+V_{c}(r)+H^{\prime}(r) .
$$

This Hamiltonian can be written in the more familiar form

$$
H=H^{0}+H^{\prime}
$$


TABLE III. CHA ground state energy $E_{10}^{(1)}$, and first-order correction due to the finite nucleus, $E_{10}^{(1)}\left(R_{c}\right)$, as a function of the confinement radius $R_{c}$ for $V_{0}=10$. Also shown is the ratio between the energy correction of the confined system, $E_{10}^{(1)}\left(R_{c}\right)$, and the correction of the free hydrogen atom $E_{10}$. Energies are in hartrees and distances are in bohrs.

\begin{tabular}{cccc}
\hline$R_{c}$ & $E_{10}$ & $E_{10}^{(1)}\left(10^{-10}\right)$ & $E_{10}^{(1)}\left(R_{c}\right) / E_{10}^{(1)}$ \\
\hline 0.3 & 9.846148432090808 & 9.930542047590157 & 10.007 \\
0.5 & 5.639829938566619 & 17.491618217286550 & 17.626 \\
0.6 & 4.097374126091258 & 13.822727567461880 & 13.929 \\
0.7 & 2.995752750577570 & 10.917393851422899 & 11.001 \\
0.9 & 1.620350180030345 & 7.179161687379589 & 7.234 \\
1.0 & 1.185777102316168 & 5.992750426662855 & 6.038 \\
1.5 & 0.116618456909048 & 3.048420555184699 & 3.071 \\
2.0 & -0.240385375166330 & 1.999717840968987 & 2.015 \\
2.5 & -0.382937517117798 & 1.527421154803173 & 1.539 \\
3.0 & -0.445545908145495 & 1.285799849400771 & 1.295 \\
3.5 & -0.474425028203553 & 1.153976852962736 & 1.162 \\
4.0 & -0.488027526391939 & 1.080287038138810 & 1.088 \\
4.5 & -0.494454829661503 & 1.039165583170746 & 1.047 \\
5.0 & -0.497468459482318 & 1.016626789889568 & 1.024 \\
\hline
\end{tabular}

TABLE IV. CHA ground state energy $E_{10}^{(1)}$, and first-order correction due to the finite nucleus, $E_{10}^{(1)}\left(R_{c}\right)$, as a function of the confinement radius $R_{c}$ for $V_{0}=\infty$. Also shown is the ratio between the energy correction of the confined system, $E_{10}^{(1)}\left(R_{c}\right)$, and the correction of the free hydrogen atom $E_{10}$. Energies are in hartrees and distances are in bohrs.

\begin{tabular}{cccc}
\hline$R_{c}$ & $E_{10}$ & $E_{10}^{(1)}\left(10^{-10}\right)$ & $E_{10}^{(1)}\left(R_{c}\right) / E_{10}^{(1)}$ \\
\hline 0.5 & 14.747970030350280 & 54.536609722874516 & 54.956 \\
0.6 & 9.527707806146348 & 33.740936784485875 & 34.000 \\
0.7 & 6.469926127251262 & 22.720335291772468 & 22.895 \\
0.8 & 4.543380181009424 & 16.278628704347120 & 16.403 \\
0.9 & 3.262189536240119 & 12.229690823293298 & 12.323 \\
1.0 & 2.373990866103664 & 9.538267646127938 & 9.611 \\
1.5 & 0.437018065247256 & 3.969102513288022 & 3.999 \\
2.0 & -0.125000000000000 & 2.355893659642013 & 2.374 \\
2.5 & -0.334910185427921 & 1.695773616320432 & 1.708 \\
3.0 & -0.423967287733454 & 1.3742119985517624 & 1.384 \\
3.5 & -0.464357128440197 & 1.202793634385484 & 1.212 \\
4.0 & -0.483265302078022 & 1.107644656981758 & 1.116 \\
4.5 & -0.492205427798878 & 1.054376061163177 & 1.062 \\
5.0 & -0.496417006591452 & 1.024902478009865 & 1.032 \\
\hline
\end{tabular}

From Eq. (17) we immediately identify the first two terms as the Hamiltonian of a hydrogen atom confined in a penetrable spherical cavity, as analyzed in previous section. We note that for $H^{0}$, the unperturbed Hamiltonian, the eigenfunctions $\psi_{n l m}^{0}$ and eigenvalues $E_{n l}^{0}$ are well-known.
The perturbation is given by $[6,21-23]$

$H^{\prime}=\left\{\begin{array}{cc}\frac{1}{2 r_{0}}\left[\left(\frac{r}{r_{0}}\right)^{2}-3\right]+\frac{1}{r}, & r<r_{0}, \\ 0, & r_{0}<r<\infty\end{array}\right.$.

As mentioned above, we are only interested in states with $l=0$, because the electron density is non-zero at the origin. 
The correction of the energy to first-order is given by

$$
E_{n_{0}}^{(1)}=\left\langle\psi_{n_{00}}\left|H^{\prime}\right| \psi_{n_{00}}\right\rangle .
$$

A straightforward calculation gives the following expression. The eigenfunctions $\psi_{n_{00}}$ are an orthonormal set of wave functions with the form

$$
\psi_{n_{00}}(r, \theta, \phi)=R_{n_{0}}(r) Y_{0}^{0}(\theta, \phi) .
$$

Substituting (21) in (20) we obtain

$$
E_{n 0}^{(1)}=\frac{1}{2 r_{0}} \int_{0}^{r_{0}}\left|R_{n 0}(r)\right|^{2}\left[\left(\frac{r}{r_{0}}\right)^{2}-3+\frac{2 r_{0}}{r}\right] r^{2} d r .
$$

In the region $r \leq r_{0}$, taking in account that for a proton, $r_{0} \ll 1$,

$$
R_{n l}(r) \cong R_{n l}(0) .
$$

Equation (22) then becomes

$$
E_{n 0}^{(1)}=\frac{r_{0}^{2}}{10}\left|R_{n 0}(0)\right|^{2} .
$$

In this work we will use the most recent measured proton radius $r_{0}=0.8335$ femtometers $\left(1.575086726 \times 10^{-5}\right.$ bohrs) [24].

\section{Shannon entropy}

Claude E. Shannon in 1948 introduced the so called Shannon entropy in his paper "A Mathematical Theory of Communication" [27]. The quantum version of Shannon entropy $S_{r}$, in configuration space is defined as

$$
S_{r}=-\int \rho(\vec{r}) \ln \rho(\vec{r}) d \vec{r},
$$

where $\rho(\vec{r})$ is probability density of the electron, in atomic units.

The Shannon entropy in quantum computation means the absolute limit of the best possible lossless compression of any communication, under some particular constraints [33]. On the other hand, the Shannon entropy has a wide variety of applications in Physics and Chemistry [34]. Usually, it is interpreted as the uncertainty associated with the particle position, which is related with the degree of localization (delocalization) of the particle. In chemistry, the Shannon entropy is associated with the delocalization of an electron in aromatic compounds [35].

Panos et al. [36], computed and plotted $S_{r}$ as a function of the atomic number, $Z$, for many-electron atoms. They found that the curve $S_{r}(Z)$ has relative minimum values for the closed shell atoms as $\mathrm{He}, \mathrm{Ne}, \mathrm{Ar}$ and $\mathrm{Kr}$. They interpreted this fact as the electronic density of those atoms is more compact (localized) than their near neighbors. Recently, this interpretation for the Shannon entropy was used successfully in the study of many-electron atoms confined in soft spherical cavities $[37,38]$. In this work we will adopt this interpretation for the Shannon entropy.

\section{Results}

In Tables I-III, we show the corrections to the ground-state energy of CHA, taking into account a nucleus with finite size. The correction to the energy is small compared with the energy of the unperturbed confined atom. As the confinement radius $R_{c}$ grows, $E_{10}^{(1)}$ approaches the value of the first-order correction of the free hydrogen atom, $E_{10 \text { free }}^{(1)}=$ $0.9923592777 \times 10^{-10}$ hartrees, according to Eq. (24).We can see that there is a change in the value of the energy correction that depends on $R_{c}$ and $V_{0}$. This behavior can be seen more clearly in Fig. 1, where we have plotted the ratio between the correction of the ground state energy of $\mathrm{CHA}$ due to the finite nucleus, relative to the correction for the free hydrogen atom. It should be noted that this ratio is independent of the value of $r_{0}$ and depends only on the ratio of the wave functions evaluated at the origin

$$
\frac{E_{10}^{(1)}}{E_{10 \text { free }}^{(1)}}=\frac{\left|R_{10}(0)\right|^{2}}{\left|R_{10 \text { free }}(0)\right|^{2}},
$$

where $R_{\text {free }}(r)$ and $R_{10}(r)$ are the radial wave functions of the free hydrogen atom and the CHA, respectively.

For a spherical cavity with impenetrable walls, the correction to the energy grows rapidly as $R_{c}$ tends to zero because by reducing $R_{c}$ the electron is closer to the nucleus without the possibility to escape. In Fig. 1 we can see that for a value of $R_{c}=1$, the energy correction $E_{10}^{(1)}$ to the ground state of the CHA is 10 times greater than in the free hydrogen atom.

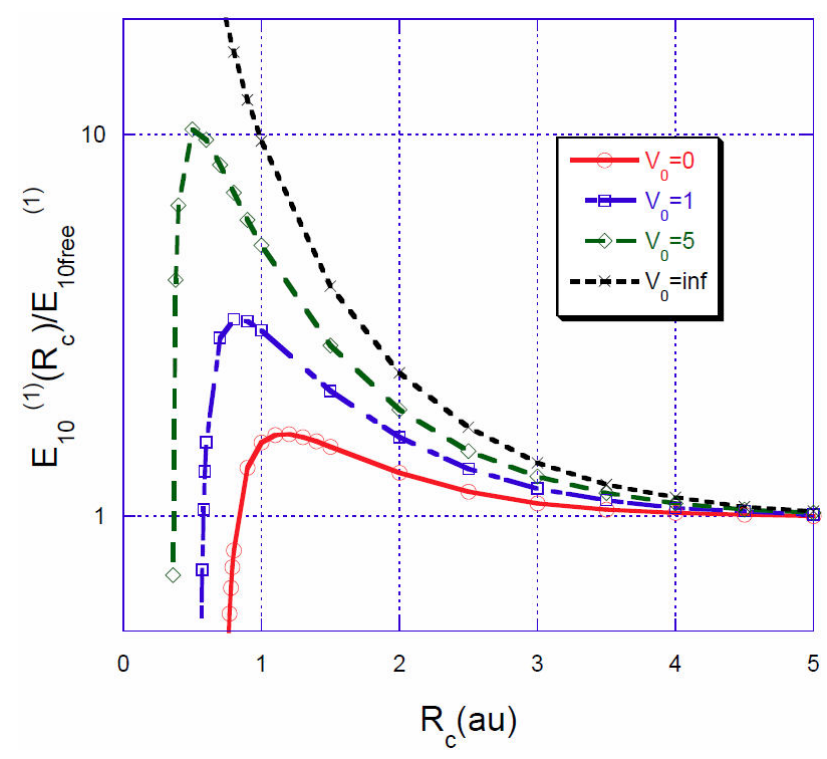

FIGURE 1. Ratio $E_{10}^{(1)}\left(R_{c}\right) / E_{10 \text { free }}^{(1)}$ of the energy correction of $\mathrm{CHA}$ to the free hydrogen atom as a function of $R_{c}$. 


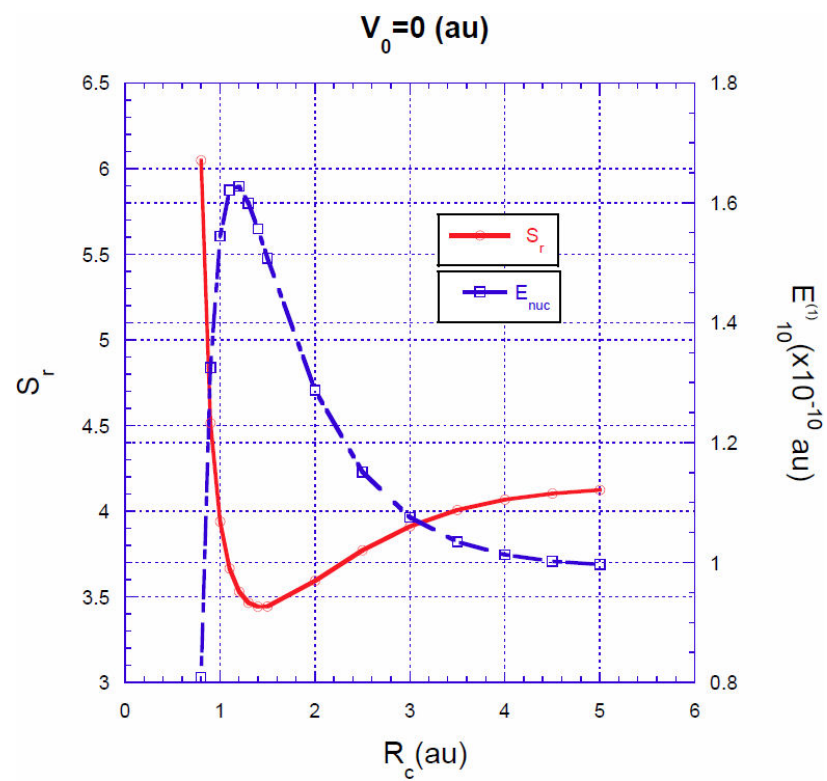

FIGURE 2. Energy correction $E_{10}^{(1)}$ and Shannon entropy $S_{r}$ as a function of $R_{c}$ for $V_{0}=0$ hartrees.

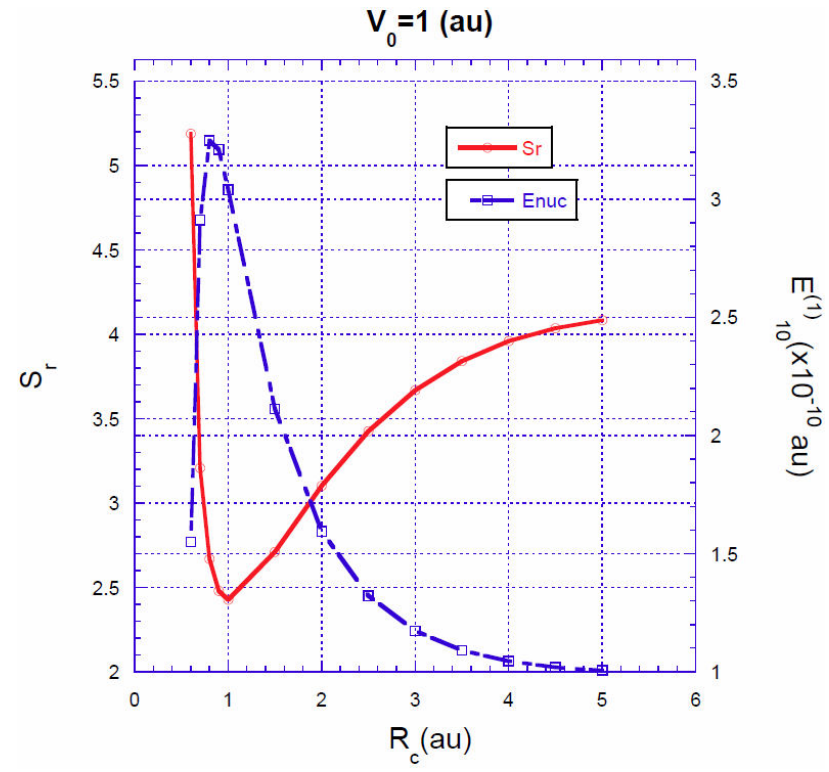

FIGURE 3. Energy correction $E_{10}^{(1)}$ and Shannon entropy $S_{r}$ as a function of $R_{c}$ for $V_{0}=1$ hartrees.

This value is in complete agreement with that calculated by Goldman and Joslin [6]. They found that for very small values of $R_{c}$ and very excited states, the correction of the energy, $E_{10}^{(1)}$, can be several orders of magnitude greater than the value for the free atom.

For a fixed value of the barrier height $V_{0}$, the situation is quite different. The energy correction $E_{10}^{(1)}\left(R_{c}\right)$ grows as $R_{c}$ decreases, and it reaches its maximum value at a confinement radius that we call $R_{c_{\max }}$. As $R_{c}$ continues to decrease, the energy correction, $E_{10}^{(1)}\left(R_{c}\right)$, decreases also, approaching zero as $R_{c}$ approaches a critical radius, at which the total energy of the electron is equal to the height of the barrier $V_{0}$.

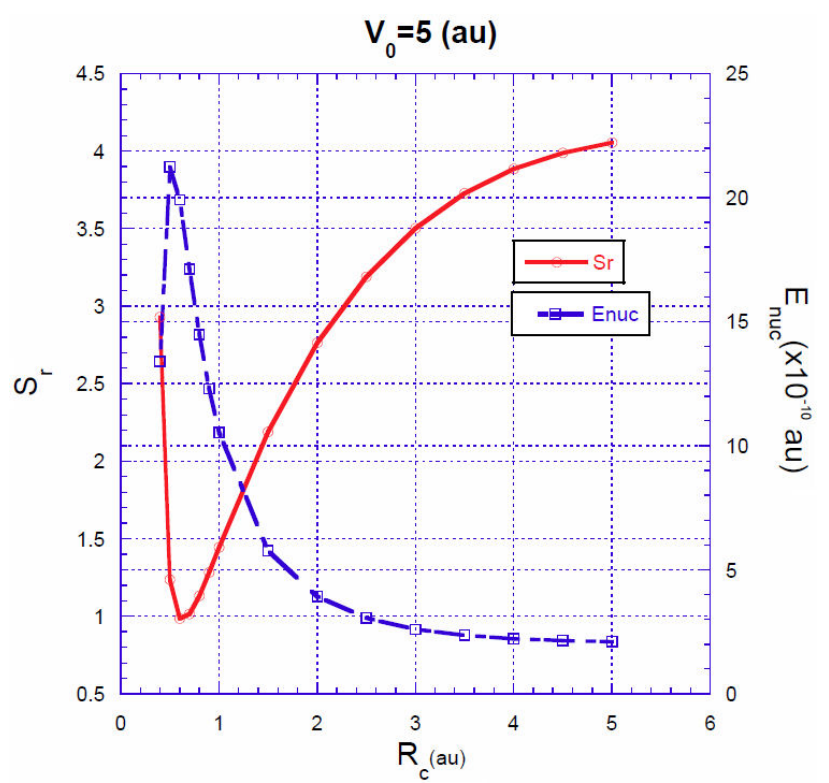

FIGURE 4. Energy correction $E_{10}^{(1)}$ and Shannon entropy $S_{r}$ as a function of $R_{c}$ for $V_{0}=5$ hartrees.

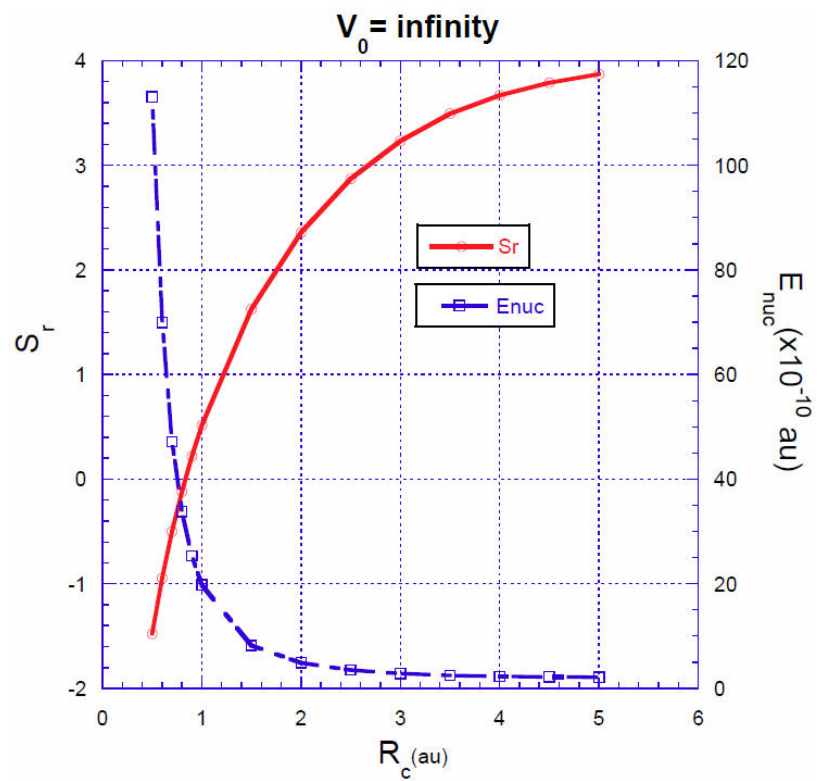

FIGURE 5. Energy correction $E_{10}^{(1)}$ and Shannon entropy $S_{r}$ as a function of $R_{c}$ for a spherical impenetrable of radius $R_{c}$.

Intuitively, one expects that the energy correction $E_{10}^{(1)}$ be higher in a small size cavity, in which the electron is closer the nucleus, i.e. a cavity in which the electronic density is more compact. One way to quantify the compactness of the electronic density is by means of the Shannon entropy [2738], as was mentioned above. This idea is supported in the interpretation of localization-delocalization associated with the Shannon entropy, $S_{r}$. A small value of $S_{r}$, means that the electron density is more localized around the nucleus [3032,36-38]. At $R_{c}^{*}$, where the entropy curve, $S_{r}\left(R_{c}\right)$, has a minimum value, the electron density is more compact around the nucleus, and thus the contribution to the energy correction 
$E_{10}^{(1)}\left(R_{c}\right)$ is greater. In Figs. 2 to 5 we plot the energy correction $E_{10}^{(1)}\left(R_{c}\right)$ and the Shannon entropy $S_{r}\left(R_{c}\right)$ for a few values of $V_{0}$ as a function of $R_{c}$. In Fig. 2 we plot together $E_{10}^{(1)}\left(R_{c}\right)$ and $S_{r}\left(R_{c}\right)$, as a function of the confinement radius, $R_{c}$, for $V_{0}=\infty$. We see that when $R_{c}$ decreases, $S_{r}$ also decreases. This means that the electron density around the nucleus increases and there is an increase in $E_{10}^{(1)}$. This behavior continues as $R_{c}$ diminishes.

In Figs. 3-5 we show the energy correction $E_{10}^{(1)}\left(R_{c}\right)$ and the Shannon entropy $S_{r}$ for fixed values of $V_{0}$. In all figures, we see that the curve of the energy correction $E_{10}^{(1)}\left(R_{c}\right)$ reaches a maximum value at a confinement radius $R_{c_{\max }}$ which depends on the value of $V_{0}$. We can also see that the curve of the Shannon entropy $S_{r}\left(R_{c}\right)$ reaches its minimum value at a confinement radius $R_{c}^{*}$, i.e., in a spherical box of radius $R_{c}^{*}$ the electron density is more compact around the nucleus. From Figs. 3-5 we can see that $R_{c_{\max }}<R_{c}^{*}$. This result can be interpreted in the following way. As $R_{c}$ decreases, the value of $S_{r}$ also decreases, and the density becomes more compact up to a maximum value at $R_{c}^{*}$. As $R_{c}$ continues to decrease, $S_{r}$ begins to increase and the electron starts to be delocalized, the wave function increases its value at the origin and inside the barrier. If $R_{c}$ continues decreas- ing, the value of the wave function at the origin decreases quickly, but it grows fast inside the barrier. This process continues until ionization take place.

\section{Conclusions}

In this work we calculated the energies and wave functions, with high numerical precision, for the ground state of the hydrogen atom confined in a penetrable spherical cavity. We also calculated the energy correction due to a nucleus of finite size for the CHA as a function of $R_{c}$ and $V_{0}$. For finite barriers, the curves of the energy correction reach a maximum value, while the curves of the Shannon entropy as a function of $R_{c}$ reach a minimum value. The maximum of the energy correction is always close to the position at which the Shannon entropy has its minimum value, i.e. at the position in which the electron density is most compact.

\section{Acknowledgments}

One of us (RAR) is grateful to Universidad Autónoma Metropolitana for providing the funds through a $\mathrm{PhD}$ studies scholarship.
1. A. Michels, J. de Boer and A. Bijl, Physica 4 (1937) 981.

2. E. Ley-Koo and S. Rubinstein, J. Chem. Phys. 71 (1979) 351.

3. D. Suryanarayana and J. A. Weil, J. Chem. Phys. 64 (1976)510.

4. J. A. Weil, J. Chem. Phys. 71 (1979) 2803.

5. F. M. Fernández and E. A. Castro, Kinam 4 (1982) 193; P. O. Fröman, S. Yngve and N.J. Fröman, J. Math. Phys. 28 (1987) 1813; S. Yngve, J. Math Phys. 29 (1988) 931; W. Jaskálski, Phys. Rep. 271 (1996) 1; A. L. Buchachenko, J. Phys. Chem. 105 (2001) 5839; J. P. Connerade, V. H. Dolmatov and P. A. Lakshmi, J. Phys. B 33 (2000) 251; J. R. Sabin, E. Brändas and S. A. Cruz (eds) The Theory of Confined Quantum Systems, Parts I and II Advances in Quantum Chemistry vol 57, (Amsterdam: Academic2009); K. D. Sen (ed) Electronic Structure of Quantum Confined Atoms and Molecules (Heidelberg: Springer2014) and references therein.

6. S. Goldman and C. Joslin, J. Phys. Chem. 96 (1992) 6021.

7. N. Aquino, Adv. Quantum Chem. 57 (2009) 148.

8. N. Aquino, G. Campoy and H. E. Montgomery, Int. J. Quantum Chem. 107 (2007) 1548.

9. H. E. Montgomery and K. D. Sen, Phys. Lett. A 376 (2012) 1992.

10. J. Hunt, J. Martin, V. Rosing, J. Winner and H. E. Montgomery, Chem. Educator 19 (2014) 384.

11. R. Cabrera-Trujillo and S. A. Cruz, Phys. Rev. A 87 (2013) 012502.

12. M. Rodríguez-Bautista, C. Díaz-García, A. M. NavarreteLópez, R. Vargas and J. Garza, J. Chem. Phys. 143 (2015) 34103.
13. M. A. Martínez-Sánchez, M. Rodríguez-Bautista, R. Vargas and J. Garza, Theor. Chem. Acc. 135 (2016) 207.

14. A. Ray, P. Das, S. K. Saha, A. Goswami and A. De, Phys. Lett. B 679 (2009) 106.

15. W. K. Hensley, W. A. Basset and R. J. Huizenga, Science Lett. 181 (1973) 1164.

16. L. Liu, C. Huh, Earth and Planetary Science Lett. 180 (2000) 163.

17. A. Ray, P. Das, N. Aquino and M. Lozano Unpublished data

18. NIST Digital Library of Mathematical Functions. http://dlmf. nist.gov/, Release 1.0.18 of 2017-09-18 F.W.J. Olver et al., eds, Sec. 13 .

19. E. Ley-Koo, E. Castaño, D. Finotello, E. Nahmad-Achar and S. Ulloa, Am. J. Phys. 48 (1980) 949.

20. Pyarelal and P. L. Bhatnagar, http://www.insa.nic.in/writerea ddata/UpLoadedFiles/PINSA/Vol18_1952_3_Art06.pdf

21. A. A. Sokolov, Y. M. Loskutov and I. M. Ternov, Quantum Mechanics (Holt Rinehart and Winston, Inc: New York 1966) p. 334.

22. C. Cohen-Tannoudji, Quantum Mechanics vol. 2 (John Wiley and Sons Inc: New York 1977) p.1141.

23. B. H. Bransden and C. J. Joachain, Physics of atoms and molecules (Longman Group UK, Ltd: London 1990) p. 245.

24. A. Beyer et al., Science 358 (2017) 79.

25. A. Adamu and Y. H. Ngadda, Int. J. Theo. Math. Phys. 7 (2017) 9. 
26. B. Holdomand and R. Koniuk, arXiv:1710.01697v1, 2017 [physics.atom-ph].

27. C. E. Shannon, Bell Syst. Tech. J., 27 (1948) 379-473; 27 (1948) 623-656. Reprinted in C. E. Shannon; W. Weaver, The Mathematical Theory of Communication; Illinois Press: Urbana, 1949); pp. 29-125 and http://cm.belllabs.com/cm /ms/what/shannonday/paper.html

28. K. D. Sen, J. Chem. Phys. 12 (2005) 074110 and references therein.

29. A. Nagy, K. D. Sen and H. E. Montgomery, Phys. Lett. A 373 (2009) 2552.

30. I. Bialinicki-Birula and L. Rudnicki, Statistical Complexity, Applications in Electronic Structure(Springer:London, 2011) Chapter 1.

31. H. G. Laguna, H. H. Corso, and R. P. Sagar, J. Math. Chem. 50 (2012) 233. H. G. Laguna, H. H. Corso, E. Castaño and R. P. Sagar, J. Math. Chem. 51 (2012) 179.
32. N. Aquino, A. Flores-Riveros and F. J. Rivas-Silva, Phys. Lett. A 377 (2013) 2062, and references therein.

33. M. A. Nielsen and I. L. Chuang, Quantum Computation and Quantum Information, Cambridge University Press, Cambridge, U. K. (2005).

34. S. R. Gadre, Phys. Rev. A 30 (1984) 620.

35. Z. Chen, C. S. Wannere Corminboeuf, R. Pucha, P. V. R. Schleyer, Chem. Rev. 105 (2005) 3842.

36. K. D. Sen (Ed.), Statistical Complexity, Applications in Electronic Structure, (Springer, London, 2011), Chap. 3.

37. M. A. Martínez-Sánchez, N. Aquino, R. Vargas and J. Garza, Chem. Phys. Lett. 90 (2017) 14.

38. M. Rodríguez-Bautista, R. Vargas, N. Aquino and J. Garza, Int. J. Quantm Chem. (2017); e25571. https://doi.org/10.1002/qua. $\underline{25571}$ 


\title{
Role of the cut-off function for the ground state variational wavefunction of the hydrogen atom confined by a hard sphere
}

\author{
R.A. Rojas and N. Aquino* \\ Departamento de Física, Universidad Autónoma Metropolitana-Iztapalapa, \\ Avenida San Rafael Atlixco 186, Col. Vicentina, Iztapalapa, 09340 Ciudad de México \\ *e-mail:naa@xanum.uam.mx
}

Received 23 July 2018; accepted 20 October 2018

\begin{abstract}
A variational treatment of the hydrogen atom in its ground state, enclosed by a hard spherical cavity of radius $R_{c}$, is developed by considering the ansatz wavefunction as the product of the free-atom $1 \mathrm{~s}$ orbital times a cut-off function to satisfy the Dirichlet boundary condition imposed by the impenetrable confining sphere. Seven different expressions for the cut-off function are employed to evaluate the energy, the cusp condition, the Shannon entropy, $\left\langle r^{-1}\right\rangle,\langle r\rangle,\left\langle r^{2}\right\rangle$, and the critical cage radius, as a function of $R_{c}$ in each case. We investigate which of the proposed cut-off functions provides best agreement with available corresponding exact calculations for the above quantities. We find that most of these cut-off functions work better in certain regions of $R_{c}$, while others are identified to give bad results in general. The cut-off functions that give, on average, better results are of the form $\left(1-\left(r / R_{c}\right)^{n}\right), n=1,2,3$.
\end{abstract}

Keywords: Confined hydrogen atom; cut-off function; Dirichlet boundary conditions.

PACS: 31.15.B; 31.15.ac; 31.15.xt

\section{Introduction}

In the middle 1930's, shortly after the formulation of quantum mechanics, Michels, de Boer and Bijl [1] proposed to study how the polarizability of the hydrogen atom would change when subjected to high pressures. They developed a model consisting of a hydrogen atom with its nucleus centrally located inside a confining impenetrable sphere of radius $R_{c}$. The impenetrable character of the sphere boundary represents, as first approximation, the repulsive potential due to all negative charges surrounding the hydrogen atom [2]. Under these conditions, the wavefunction of the particle must vanish at the walls, satisfying the Dirichlet boundary condition. This simple model predicted qualitative results that explain some experimental results and, over the years, it became one of the most successful models about the study of confined quantum systems [2-10]. Confined quantum systems are used to study a great variety of problems of physics and chemistry [1-20]. For example, the effects on electronic structure of atoms and molecules trapped in fullerenes [13] and in other microscopic cavities, the study of artificial systems built within semiconductors, such as quantum wells, wires, and dots $[11,12,14]$. Other applications for confined quantum systems are: the study of specific heat of a crystal subjected to an external pressure [15], spectroscopic data for astrophysics [16], matter inside electromagnetic fields [17], nuclear models [18], etc.

The model of the confined hydrogen atom proposed by Michels et. al. [1], is the following: a hydrogen atom is boxed in a spherical impenetrable cavity with the nucleus clamped at the center of the sphere, and the electron is moving within the volume of the sphere. The impenetrable walls impose Dirichlet condition over the wave functions on the surface $\delta \Omega$ of the sphere.

$$
\left.\psi(\vec{r})\right|_{\vec{r} \in \delta \Omega}=0
$$

This simple model has been widely used to test new techniques to solve the Schrödinger equation (exact solutions) or to explore new trial variational wavefunctions to compare with the most accuarte calculations [10]. One of the approximate methods is the direct variational method (DVM), in which the trial wavefunction is constructed as the product of a wave function, similar to the wave function of the free (unbounded) system, times a non-singular function $f_{\text {cut }}$, which vanishes on the boundary of the box $\delta \Omega$.

The selection of the cut-off term $f_{\text {cut }}$ in the literature has been arbitrary. Some authors have used different forms of cut-off function: linear [5-7], exponential [19], or (1 $\left.r / R_{c}\right)^{n}$, where $n$ is a positive integer number [20], etc. On the other hand, to our knowledge there is no systematic study about the effect of the cut-off function on the energy of the confined hydrogen atom, obtained in a variational way.

The objetive of this work is to explore several criteria to decide which of the cut-off functions is the best. We tested seven trial wavefunction constructed as a product of the free 1s hydrogen-like orbital times seven different cut-off function $f_{\text {cut }}$. We compared the calculated physical quantities with the exact ones $[9,10,33]$ to decide which of the trial wavefunctions give the best aproximations.

The organization of this work is as follows: in Sec. 2 we present the methodology used to solve the confined hydrogen atom (CHA) problem within spherical impenetrable cavity using the direct variational method. We used seven different cut-off functions to compute the ground state energy and several expectation values of $r$ as functions of the confinement radius. In Sec. 3 we compute the Shannon entropy in coordinate space for the different trial wave functions of Sec. 2. In 
Sec. 4 we compare the results obtained by using the different trial wave functions with the accurate numerical results $[9-10,33]$. Finally in Sec. 5 we give our conclusions.

\section{The ground state energy of the CHA by us- ing different cut-off functions}

The Hamiltonian of the confined hydrogen atom, in atomic units, is given by

$$
\begin{aligned}
H & =-\frac{1}{2} \nabla^{2}-\frac{1}{r}+U_{c}(r) \\
U_{c}(r) & =\left\{\begin{array}{cc}
0, & r \leq R_{c} \\
\infty, & r>R_{c}
\end{array}\right.
\end{aligned}
$$

In the DVM, the ground state wavefunction $\psi_{t}$ of the CHA is the product of a function $\psi_{f}$, similar to the $1 \mathrm{~s}$ orbital of the free hydrogen atom, times a nonsingular cut-off function $f_{\text {cut }}$, such that $f_{\text {cut }}\left(r=R_{c}\right)=0$.

$$
\psi_{t}=\psi_{f} f_{\text {cut }} .
$$

In this way $\psi_{t}$ satisfies the Dirichlet boundary condition of the problem

$$
\psi_{t}\left(r=R_{c}, \theta, \varphi\right)=0 .
$$

We propose the wave function $\psi_{f}$ as the $1 \mathrm{~s}$ hydrogen-like wavefunction:

$$
\psi_{f}(r)=A e^{-\alpha r},
$$

where $A$ is the normalization constant and $\alpha$ is the variational parameter.

The trial wavefunction for the ground state of the CHA, with its nucleus clamped at the origin of a sphere of radius $R_{c}$ is given by:

$$
\psi_{t}(r, \theta, \varphi)=A e^{-\alpha r} f_{c u t}(r),
$$

This function must be a decreasing function of $r$ in the interval $\left[0, R_{c}\right]$ and it is valid for negative and positive energy values.

We use this trial wave function (5) and the variational method to minimize the energy functional $E(\alpha)$, wich is given by:

$$
E(\alpha)=\frac{\left\langle\psi_{t}|H| \psi_{t}\right\rangle}{\left\langle\psi_{t} \psi_{t}\right\rangle}
$$

As we mentioned above the cut-off function $f_{\text {cut }}$ is, in principle, arbitrary. We selected seven different cut-off functions to evaluate the quality with which they reproduce the energy of the CHA ground state and several expectation values of $r$. The following seven trial wavefunctions are constructed by using the rule given by the Eq. (5) :

I

$$
e^{-\alpha r}\left(1-\frac{r}{R_{c}}\right)
$$

II

$$
e^{-\alpha r}\left(1-\left(\frac{r}{R_{c}}\right)^{2}\right) \text {. }
$$

III

$$
e^{-\alpha r}\left(1-\left(\frac{r}{R_{c}}\right)^{3}\right)
$$

IV

$$
e^{-\alpha r}\left(1-\left(\frac{r}{R_{c}}\right)^{4}\right)
$$

V

$$
e^{-\alpha r}\left(1-\frac{r}{R_{c}}\right)^{2}
$$

VI

$$
e^{-\alpha r} j_{0}\left(\frac{X_{10}}{R_{c}} r\right)
$$

VII

$$
e^{-\alpha r}\left(c_{1} j_{0}\left(\frac{X_{10}}{R_{c}} r\right)+c_{2} j_{0}\left(\frac{X_{20}}{R_{c}} r\right)\right)
$$

where $j_{0}$ is the zeroth-order spherical Bessel function. $X_{10}=$ $\pi$ and $X_{20}=2 \pi$ are the first and second zeros of the spherical Bessel function, $c_{1}$ and $c_{2}$ are linear variational parameters, respectively. $j_{0}$ is the wave function, with angular momentum $l=0$, of the free particle in a spherical impenetrable box. The wave functions $j_{0}\left(X_{10} / R_{c} r\right)$ and $j_{0}\left(X_{20} / R_{c} r\right)$, are the gound state and the first excited state wave function of the free particle in a box, respectively. The wavefunction VII is the linear superposition of the two linearly independent wavefunctions.

We must note that all the differences in the calculated values of physical properties are due to the kind of cut-off function used.

According to the variational theorem, we must calculate the expectation value of the energy using the trial wavefunction. Thus, because of the symmetry of the problem, the integrals we need to calculate are:

$$
\left\langle\psi_{t}|H| \psi_{t}\right\rangle=\frac{\left\langle\psi_{t}|T| \psi_{t}\right\rangle+\left\langle\psi_{t}|V| \psi_{t}\right\rangle}{\left\langle\psi_{t} \mid \psi_{t}\right\rangle}
$$

Where

$$
\begin{aligned}
\left\langle\psi_{t}|V| \psi_{t}\right\rangle & =-\int_{0}^{R_{c}}\left[e^{-\alpha r} f_{\text {cut }}(r)\right]^{2} r d r \\
\left\langle\psi_{t}|T| \psi_{t}\right\rangle & =-\frac{1}{2} \int_{0}^{R_{c}} e^{-\alpha r} f_{\text {cut }}(r) \\
& \times\left\{\frac{1}{r^{2}} \frac{d}{d r}\left[r^{2} \frac{d}{d r}\left(e^{-\alpha r} f_{\text {cut }}(r)\right)\right]\right\} r^{2} d r
\end{aligned}
$$


TABLE I. Ground state energy for the CHA, with seven different trial wavefunctions (see the text) by means of the variational method. These results are compared with the exact value [10]. Distances are in Bohrs, while energies are in Hartrees.

\begin{tabular}{ccccccccc}
\hline$R_{c}$ & $\mathrm{I}$ & $\mathrm{II}$ & $\mathrm{III}$ & $\mathrm{IV}$ & $\mathrm{V}$ & $\mathrm{VI}$ & $\mathrm{VII}$ & Exact value \\
\hline 0.5 & 14.8971 & 14.8152 & 14.8774 & 15.0390 & 16.1512 & 14.7621 & 14.7619 & 14.7480 \\
0.6 & 9.6180 & 9.5657 & 9.6056 & 9.7117 & 10.4975 & 9.5419 & 9.5416 & 9.5277 \\
0.7 & 6.5272 & 6.4921 & 6.5190 & 6.5924 & 7.1791 & 6.4842 & 6.4839 & 6.4699 \\
0.8 & 4.5808 & 4.5565 & 4.5752 & 4.6279 & 5.0838 & 4.5577 & 4.5574 & 4.5434 \\
0.9 & 3.2870 & 3.2699 & 3.2831 & 3.3220 & 3.6873 & 3.2765 & 3.2762 & 3.2622 \\
1 & 2.3906 & 2.3784 & 2.3878 & 2.4170 & 2.7168 & 2.3884 & 2.3880 & 2.3740 \\
1.2 & 1.2767 & 1.2705 & 1.2753 & 1.2924 & 1.5053 & 1.2838 & 1.2833 & 1.2693 \\
1.5 & 0.4388 & 0.4371 & 0.4384 & 0.4464 & 0.5861 & 0.4515 & 0.4508 & 0.4370 \\
1.7 & 0.1396 & 0.1394 & 0.1395 & 0.1441 & 0.2541 & 0.1535 & 0.1527 & 0.1391 \\
2 & -0.1250 & -0.1240 & -0.1249 & -0.1232 & -0.0431 & -0.1108 & -0.1118 & -0.1250 \\
3 & -0.4225 & -0.4206 & -0.4224 & -0.4237 & -0.3902 & -0.4116 & -0.4132 & -0.4240 \\
4 & -0.4811 & -0.4796 & -0.4811 & -0.4824 & -0.4670 & -0.4741 & -0.4760 & -0.4833 \\
5 & -0.4947 & -0.4937 & -0.4948 & -0.4956 & -0.4884 & -0.4906 & -0.4924 & -0.4964 \\
6 & -0.4982 & -0.4976 & -0.4983 & -0.4988 & -0.4953 & -0.4959 & -0.4974 & -0.4993 \\
7 & -0.4993 & -0.4989 & -0.4994 & -0.4996 & -0.4978 & -0.4979 & -0.4990 & -0.4999 \\
8 & -0.4997 & -0.4995 & -0.4997 & -0.4999 & -0.4989 & -0.4988 & -0.4996 & -0.5000 \\
9 & -0.4998 & -0.4997 & -0.4999 & -0.5000 & -0.4994 & -0.4993 & -0.4998 & -0.5000 \\
10 & -0.4999 & -0.4998 & -0.4999 & -0.5000 & -0.4996 & -0.4996 & -0.4999 & -0.5000 \\
\hline
\end{tabular}

and the overlap integral is given by

$$
\left\langle\psi_{t} \mid \psi_{t}\right\rangle=-\int_{0}^{R_{c}}\left(e^{-\alpha r} f_{c u t}(r)\right)^{2}
$$

The energy functional as a function of the variational parameter $\alpha$ for a given confinement radius $R_{c}$ is the following:

$$
E_{\mathrm{var}}\left(\alpha ; R_{c}\right)=\left\langle\psi_{t}|H| \psi_{t}\right\rangle
$$

Minimizing $E_{\text {var }}$ respect $\alpha$ for a fixed value of $R_{c}$, we find an upper limit for the ground state energy $E$ of the CHA.

Most of the integrals involved in the calculation of the energy functional (11) are obtained in analytical form, except for the cut-off functions VI and VII. In order to minimize the energy functional, it is necessary to fix the value of $R_{c}$ and vary $\alpha$. We used the program Mathematica 9 and the command FindMinimum to obtain the minimum of Eq. (11) for each value of $R_{c}$. The optimum values of the energy for every trial wavefunction as a function of $R_{c}$ are shown in Table I. We also show the most accurate energy values [10], which we will call "the exact values".

\section{The Shannon entropy}

The Shannon entropy in coordinate space is defined as $[27,28]$

$$
S_{r}=\int_{R_{c}}^{0} d^{3} \vec{r}\left|\psi_{t}\right|^{2} \ln \left|\psi_{t}\right|^{2},
$$

where $\psi_{t}$ is normalized to one. We must remember that the trial wavefunction has the following form $\psi_{t}=$ $A e^{-\alpha r} f_{\text {cut }}(r) Y_{00}(\theta, \varphi)$, where $A$ is the normalization constant and $Y_{00}(\theta, \varphi)=1 / \sqrt{4 \pi}$.

Gadre et al. used the Shannon entropy as a measure of the quality of the basis set for free atomic and molecular systems [27,28]. In their calculations they constructed a wave function as a linear combination of functions from a basis set. They observed that, on having increased the number of functions of the basis set, the constructed wavefunction approaches better the exact wavefunction of the system, and the Shannon entropy increases approaching the Shannon entropy of the exact wavefunction [9-10,33]. According to the Maximum Entropy Principle due to Jaynes [29] one must choose the trial wavefuntion whose Shannon entropy is the highest among a set of functions that satisfy the appropriate constraints of the system. In this way, Shannon entropy could offer an alternative form to determine the quality of the trial wavefunction for confined systems. 


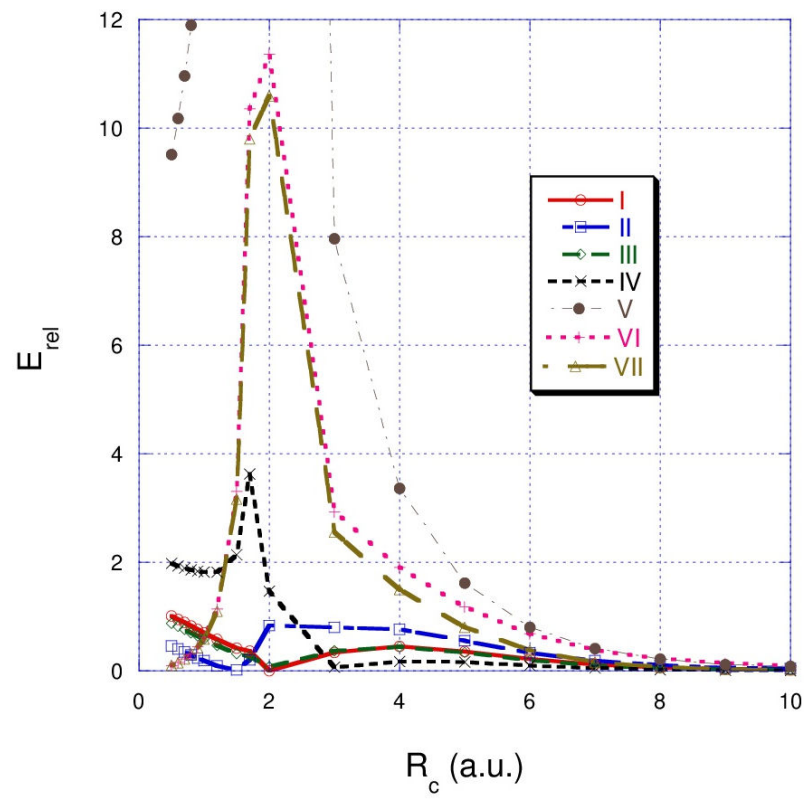

FIGURE 1. Relative percentage error in the ground state energy of $\mathrm{CHA}$ produced by the seven different trial wavefunctions as a function of the confinement radius $R_{c}$. The wavefunction $\mathrm{V}$ gives the mayor errors. Whereas the wavefunctions V and VI give the lowest errors for the strong confinement.

\section{Results}

The optimized energies as a function of $R_{c}$ for every trial wave function, appears in the Table I. We also calculate the relative percentage error defined in the following way:

$$
E_{\text {rel }}=\frac{E-E_{\text {exact }}}{E_{\text {exact }}} \times 100 .
$$

This quantity, $E_{r e l}$, is always positive because the energy calculated in a variational way $E$ is always greater than the exact energy [9-10,33]. In Fig. 1 we plotted $E_{r e l}$ vs $R_{c}$ for every trial wavefunction considered in this work.

From Table I, we see that for confinement radii less than $1.0 \mathrm{au}$, the trial wavefunction VI gives the lowest energy with, $f_{\text {cut }}(r)=j_{0}\left(\left(X_{10} r / R_{c}\right)\right.$. This can be understood because in the strong confinement regime the system behaves like a free particle inside an impenetrable spherical box [25] whose radial wavefunctions are precisely the spherical Bessel functions [26]. For confinement radii between 0.8 and 1.7 au, the trial wavefunction II gives the lowest energy. From Fig. 1 we can observe that the wavefunctions VI and VII produce an overestimation of the energy in a neighborhood of $R_{c}=2$ au. We also can observe that the trial wavefunction IV overestimates the ground state energy for $R_{c}<2$ au.

As we can see from the Fig. 1, every trial wavefunction has a region in $R_{c}$ at which it approaches better to the exact energy [9-10,33]. Nevertheless, the trial wavefunctions I - III are those that predict energies nearer to the exact value [9-10,33]. The wavefunction $\mathrm{V}$ gives the largest error in the region $0.5 \leq R_{c} \leq 4$.

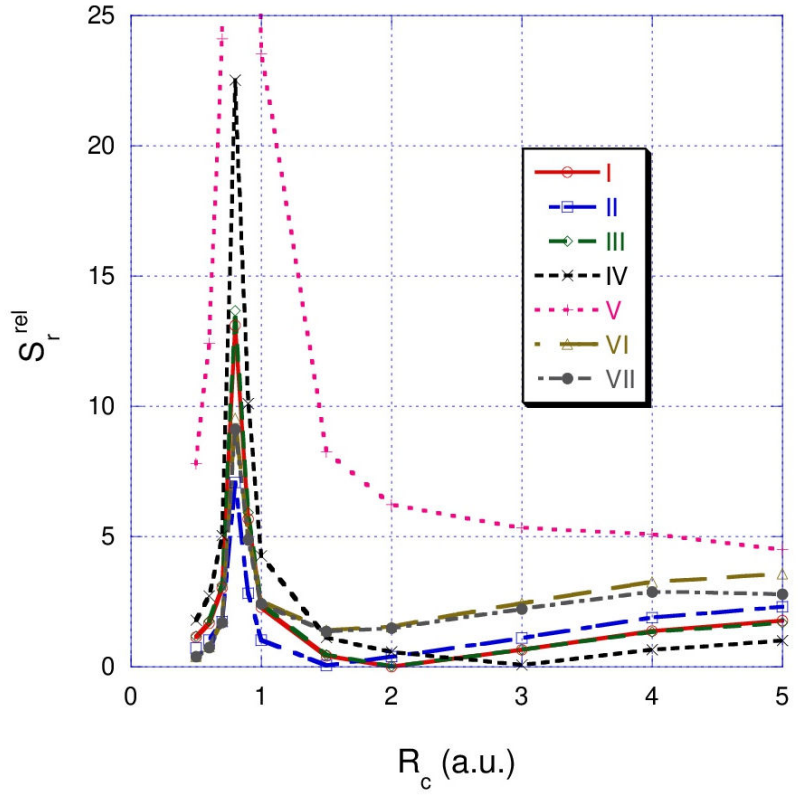

FIGURE 2. Relative percentage error in the Shannon entropy, for the CHA ground state produced by the seven different trial wavefunctions as a function of the confinement radius $R_{c}$. The wavefunction IV produces the lowest error for $R_{c}>2$, whereas the wavefunction $\mathrm{V}$ produces the highest error.

Finally, for $R_{c}$ larger than 2.0 au all trial wavefunctions give good estimations for the ground state energy. This behavior is shown graphically in Fig. 1.

We calculated the Shannon entropy for each of the trial wavefunctions. The Shannon entropy for all trial wavefunctions have smaller values than the Shannon entropy for the exact wavefunction [9-10, 33]. We define the Shannon entropy relative error $S_{r}^{r e l}$ as follows:

$$
S_{r}^{r e l}=\frac{S_{r}^{\text {exact }}-S_{r}^{\text {trial }}}{S_{r}^{\text {exact }}} \times 100,
$$

where $S_{r}^{\text {exact }}$ and $S_{r}^{\text {trial }}$, are the Shannon entropy for the exact wavefunction [9-10,33] and for any trial wavefunction, respectively. In the Fig. 2 we show the relative error $S_{r}^{r e l}$ for each of the trial wavefunctions. For the region, $0.5<R_{c}<$ $0.8 \mathrm{au}$, the trial wavefunctions that have values closer to the exact Shannon entropy [32] are I - III and VI - VII. While in the region $0.8<R_{c}<2$ au, the trial wavefunctions with less error are I - III. For values, $R_{c}>2$ au, the trial wavefunction IV has the lowest errors. Whereas the wavefunction V produces the largest errors. The maximum error produced by the all wavefunction are reached near the $R_{c}=0.8$.

In the interval, [0.5,1.5], trial wavefunction II produces small errors on the prediction of the Shannon entropy and in the energy whereas the function IV has similar behavior in for $R_{c}>5$ au. We conclude that the Gadre's conjecture is not a good criterion to decide the quality of the trial wavefunctions.

In Fig. 3 we show the cusp condition at the origin of the trial wavefunctions I-VII as a function of $R_{c}$. The cusp con- 


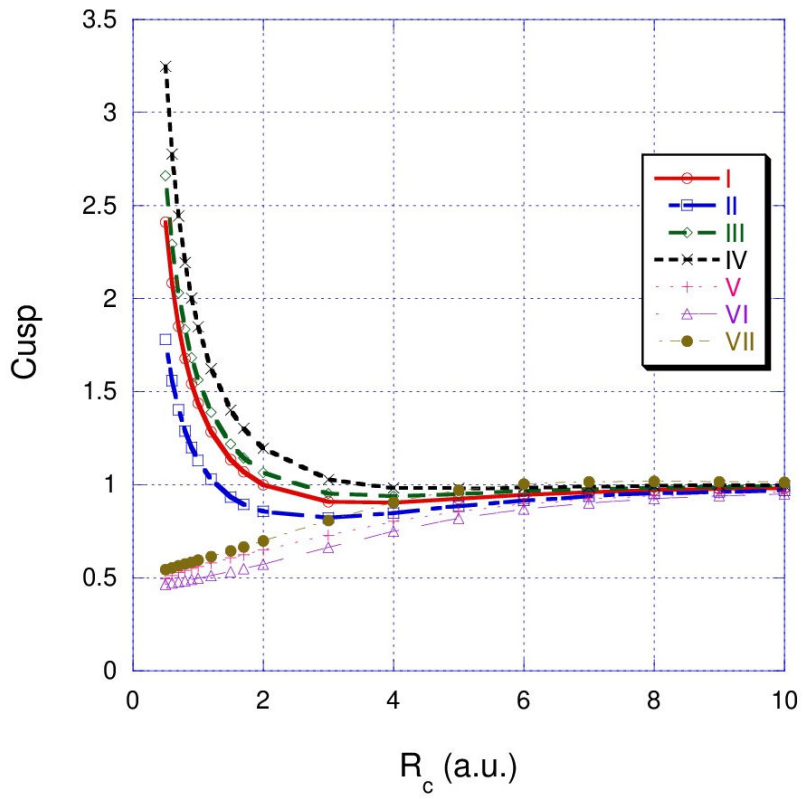

FIGURE 3. The cusp condition of CHA produced by the seven different trial wavefunctions as a function of the confinement radius $R_{c}$.

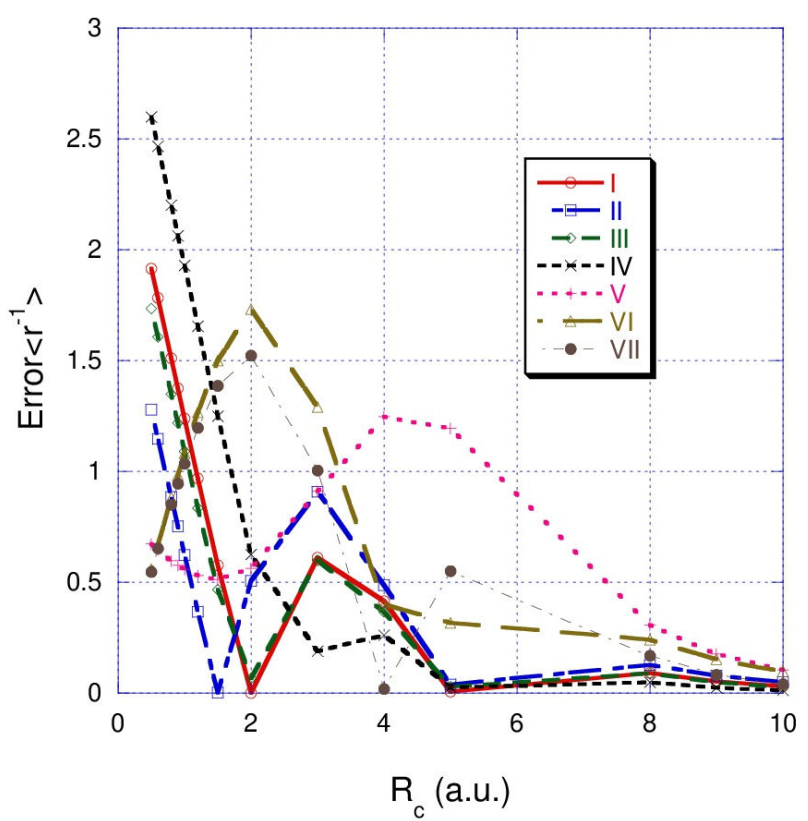

FIGURE 4. Relative percentage error of $\left\langle r^{-1}\right\rangle$ produced by the seven different trial wavefunctions as a function of the confinement radius $R_{c}$.

dition at the origin of the exact wavefunction for CHA is equal to 1 . Accordingly, the best trial wavefunction will be the one with the cusp closer to 1 . As we can see, for large confinement radius all functions satisfy this requirement. However, as the confinement radius $R_{c}$ decreases the cusp condition for all trial functions start to move away from 1. In the region, $R_{c} \leq 2$ au, this difference begins to be noticeable. The wavefunctions V, VI and VII are those closer

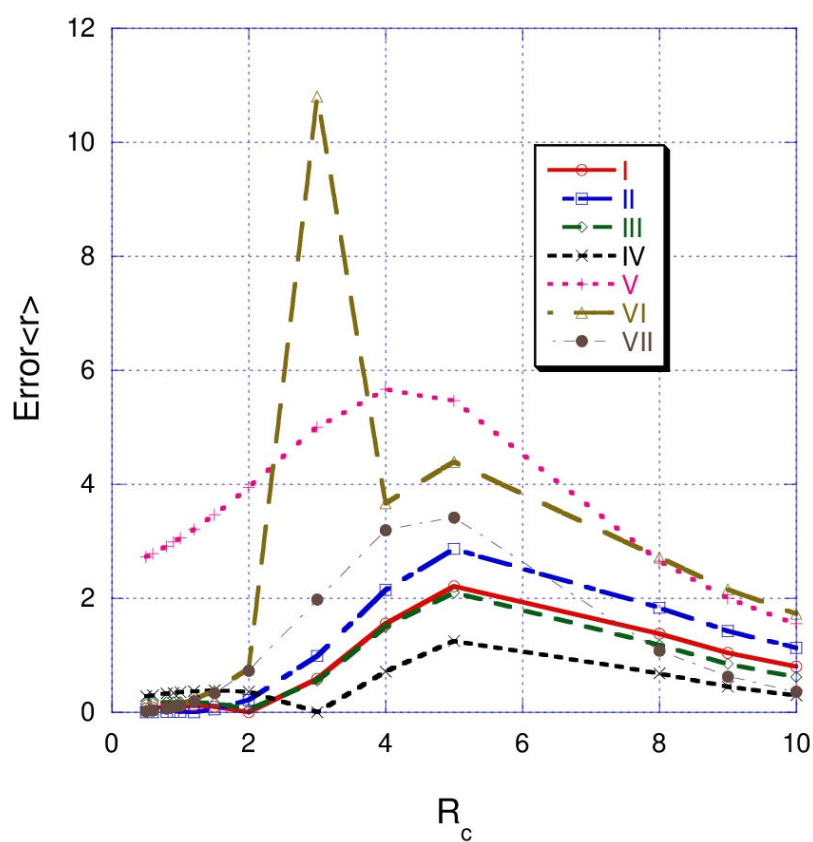

FIGURE 5. Relative percentage error of $\langle r\rangle$ produced by the seven different trial wavefunctions as a function of the confinement radius $R_{c}$. The mayor error is produced by the wavefunctions $\mathrm{V}$ and VI for $R_{c}>2$ au.

to one, reaching a value of 0.5 at $R_{c} \sim 0.5$ au. The cusp values for the rest of the trial functions are not good, few of them having a cusp value greater than 1.5 , as for example, the wavefunction I, II and IV at, $R_{c} \sim 0.5$ au.

These two criteria, the Shannon entropy and the cusp condition, are not sufficient to decide which of trial wavefunctions approximates better to the exact one. To try to give a clearer answer to this question, we need to compute few expectation position values by using the trial wave functions I-VII and comparing those results with the exact ones.

The position expectation values are given by:

$$
\begin{aligned}
\left\langle r^{n}\right\rangle & =\frac{\left\langle\psi_{t}\left|r^{n}\right| \psi_{t}\right\rangle}{\left\langle\psi_{t} \mid \psi_{t}\right\rangle} \\
& =\frac{\int_{0}^{R_{c}}\left(e^{-\alpha r} f_{c u t}(r)\right)^{2} r^{n+2} d r}{\int_{0}^{R_{c}}\left(e^{-\alpha r} f_{c u t}(r)\right)^{2} r^{2} d r}, \quad n \in Z,
\end{aligned}
$$

whereas the relative error is the following:

$$
\operatorname{Error}\left\langle r^{n}\right\rangle=\frac{\left\langle r^{n}\right\rangle-\left\langle r^{n}\right\rangle_{\text {exacto }}}{\left\langle r^{n}\right\rangle_{\text {exacto }}} \times 100
$$

The relative error for $n=-1,1$ and 2 are shown in Figs. 4-6. In Fig. 4 we show the relative error in the calculation of the expectation value $\left\langle r^{-1}\right\rangle$ as a function of the confinement radius, $R_{c}$, for all wavefunctions I-VII. For a confinement radius of $R_{c}=0.5$ au all the trial wavefunc- 


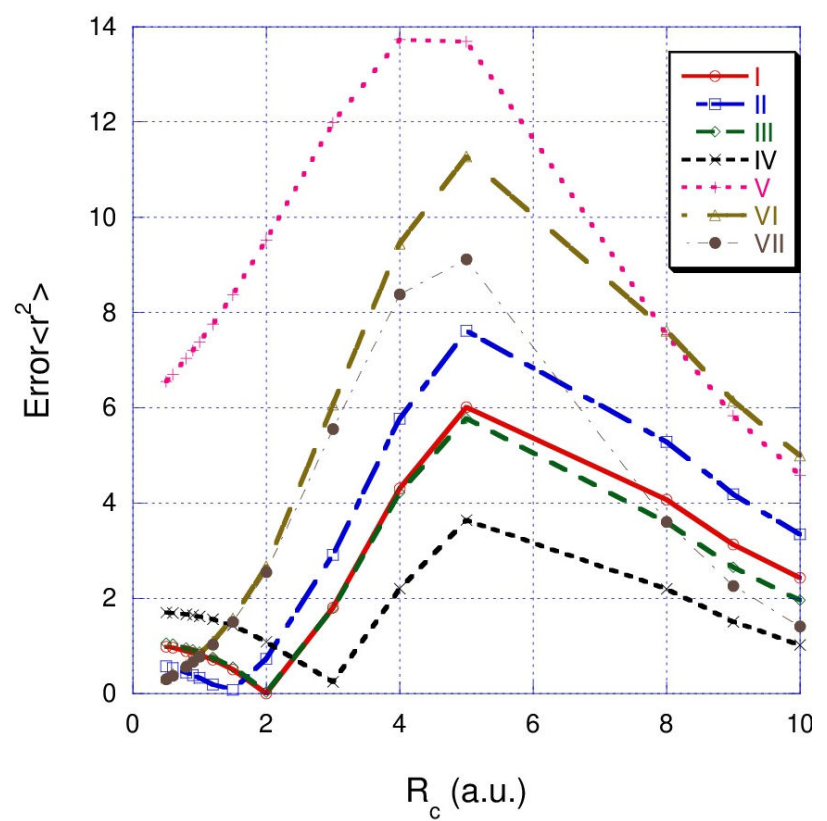

FIGURE 6. Relative percentage error of $\left\langle r^{2}\right\rangle$ produced by the seven different trial wavefunctions as a function of the confinement radius $R_{c}$. The mayor error is produced by the wavefunction $\mathrm{V}$.

tions give errors between $0.5 \%$ to $2.5 \%$, the wavefunctions V-VII have the lowest relative error. However, as the radius, $R_{c}$ increases, the percentage error produced by those trial wavefunctions also increases up to a maximum value of about $1.6 \%$. Whereas, the trial wavefunction I-III give errors smaller than $15 \%$ at, $1<R_{c}<2$ au.

In the region, $1 \leq R_{c} \leq 2$ au, the trial wavefunction II produces the lowest error of $\left\langle r^{-1}\right\rangle$. For the region $R_{c}>2 \mathrm{au}$, in general, the best results are obtained with the trial wavefunction I and III. The function IV has the highest relative error for $R_{c}<2 \mathrm{au}$. The trial wavefunction $\mathrm{V}$ produces unsatisfactory results for $2 \leq R_{c} \leq 8$ au.

Regarding the relative error of $\langle r\rangle$ we see that in the region, $0 \leq R_{c} \leq 2 \mathrm{au}$, all functions except the function of type $\mathrm{V}$, produce very small errors. In the region, $2 \leq R_{c} \leq 4 \mathrm{au}$, the trial wavefunction VI produces an error which can reach more than $10 \%$. For the region $R_{c}>2$ the trial wavefunctions I-IV and VII produce errors less than $4 \%$. While the functions $\mathrm{V}$ and VI have the largest errors.

For the relative error of $\left\langle r^{2}\right\rangle$ as a function of $R_{c}$ we found the following features. In the region, $0 \leq R_{c} \leq 2 \mathrm{au}$, all functions except the function $\mathrm{V}$ produce errors lower than $2 \%$. For the region $R_{c}>2$ au the functions VI and VII produce the largest errors, between 9-12\%, around, $R_{c}=5 \mathrm{au}$, and the error tends to diminish as $R_{c}$ grows. While the functions I-IV have errors lower than $8 \%$, and those errors tend to diminish fast as $R_{c}$ increases. The largest error is produced for the function $\mathrm{V}$.

On the other hand, there exist two additional criteria to test the variational trial wave functions I-VII. They are: the critical cage radius [30,32,34,36-37] and a degeneracy which results from choosing the radius of confinement $R_{c}$ exactly at nodes of the free hydrogen wave functions [38-40].
The confinement radius at which the CHA total energy becomes zero is called the critical cage radius $r_{c}[30,34,36]$. Sommerfeld and Welker [30], and recently Ley-Koo [37] showed that $r_{c}$ can be obtained as a function of the zeros of the Bessel function of first class $J$ of order $2 l+1$.

$$
r_{c}=\frac{1}{8}\left(\chi_{i, 2 l+1}\right)^{2},
$$

Where $\chi_{i, 2 l+1}$ denotes the $i$ th zero of $J_{2 l+1}$.

The exact value of the critical radius for the CHA ground state is equal to 1.8353 . The critical radius predicted by the trial wave functions I-VII are: $1.83,1.83,1.79,1.75,1.69$, 1.851 and 1.852 , respectively. The wave functions I-III are the best to predict a critical cage radius.

Seven decades ago de Groot and ten Seldam [38] noted that each zero of the wave function, of some state of the free hydrogen atom, is a cage size $R_{c}$ for the confined atom, and the latter has the same energy as the state of the former. For example, the $2 s$ wave function of the free hydrogen atom has a node at $r=2$ au.[39-41] The ground state energy of the hydrogen atom confined in a box $R_{c}=2 \mathrm{au}$, is $-1 / 8 \mathrm{au}$, that corresponds to the energy of the $2 s$ state of the free hydrogen atom.

The wave function of the state $2 \mathrm{~s}$ of the free hydrogen atom is

$$
\psi_{2 s}=N(2-r) e^{-r / 2}=(2 N)\left(e^{-r / 2}\right)\left(1-\frac{r}{2}\right),
$$

apart from the normalization, it is identical to the function I for $R_{c}=2$ and $\alpha=1 / 2$.

The first node of the wave function of any given state of the free hydrogen atom gives a cage of size $R_{c}$, in which case the ground state of the CHA has the same energy as the free hydrogen atom. This argument can be extended for the identification of the excited states of the CHA. However, this procedure gives the ground state energy of the CHA only for particular values of $R_{c}$.

\section{Conclusions}

In this work we used the direct variational method to compute the ground state energy of the confined hydrogen atom in an impenetrable spherical box. In this approach, the trial wavefunction is constructed as the product of the 1s hydrogen-like (free) orbital times a cut-off function. Seven different cut-off functions were used for calculations of the energy, cusp condition, Shannon entropy, $\left\langle r^{-1}\right\rangle,\langle r\rangle,\left\langle r^{2}\right\rangle$ and the critical cage radius as a function of $R_{c}$.

We found that there are regions of $R_{c}$ for which certain trial wavefunctions predict a physical property with a small error, but in other regions the same wavefunctions predict it with a high error. For example, the trial wavefunctios VI and VII, predict energy values with small errors in the region of strong confinement, $R_{c}<1$, but large error in $1 \leq R_{c} \leq 3$. On the other hand, the wavefunction I-III give small errors in the energy for the region $R_{c}>2$. It should be noted that 
the functions I and III behave in much the same way, but the calculations with the wavefunction of type I are more simple than those with the wavefunction of type III. The trial wavefunction $\mathrm{V}$ gives the largest errors in the estimation of the energy, and for this reason it is not recommended for this kind of computations.

Acording to the criterion on the degeneracy which results from choosing the confinement radius, $R_{c}$, on the radial node of the free wave function, the best function should be the wavefunction I. However, this is apparent because this wavefunction I does not behave as the wave function of the free particle, for small radii. A good trial wave function must behave as the wavefunction of a free particle in a box for small values of $R_{c}$ and like a free $1 s$ hydrogen wavefunction for large values of $R_{c}$.

The best wavefunction is one that reproduces all the physical properties of the system with the lowest error comparing with the exact ones. None of the wavefunctions studied in this work satisfy this definition. We can conclude that, the wavefunctions VI and VII are very useful for strong confinement (small $R_{c}$ ). For intermediate and large values of $R_{c}$ wave functions of types I and II are the most recommendable.

One way to construct better trial wavefunctions for this problem consists in the inclusion of more radial terms and variational parameters in the radial wavefunction. For exam- ple, Varshni [22] improved wavefunction I, as follows:

$$
\psi=\left(1-\frac{r}{R_{c}}\right) e^{-\alpha r}(1+\beta r),
$$

Where $\alpha$ and $\beta$ are variational parameters.

Whereas, Montgomery [36] proposed a generalization of Varshni's vawefunction:

$$
\psi=\left(1-\frac{r}{R_{c}}\right) e^{-\alpha r}(1+\beta r)\left(e^{-\alpha r} \sum_{p} a_{p} r^{p}\right)
$$

Where $a_{n}$, are linear variational parameters and $\alpha$ is a non-linear variational parameter.

The last trial wavefunction gives energy and other physical properties near to the exact ones [10]. We must note that Varshni [10] and Montgomery [36] used $\left(1-\left(r / R_{c}\right)\right)$ as a cut-off function.

\section{Acknowledgments}

We thank S. A. Cruz and an anonymous referee for their usefull coments. One of us (RAR) is grateful to Universidad Autónoma Metropolitana for providing the through a $\mathrm{PhD}$ studies scholarship.
1. Michels, J. De Boer and A. Bijl, Physica 4 (1937) 981.

2. N. Aquino, Advances in Quantum Chemistry 57 (2009) 123.

3. J. Gorecki, W. Byers Brown, J. Phys. B: At. Mol. Opt. Phys. 20 (1987) 5953.

4. J. Gorecki, W. Byers Brown, J. Phys. B: At. Mol. Opt. Phys. 22 (1989) 2659.

5. J. L. Marín and S. A. Cruz, J. Phys. B: At. Opt. Phys. 24 (1991) 2899-2907.

6. Y.P. Varshni, J. Phys. B: At. Mol. Opt. Phys. 30 (1997) L589.

7. E. V. Ludeña, J. Chem. Phys. 66 (1977) 468.

8. A. Sarsa, E. Buendía and F. J. Gálvez, J. Phys. B: At. Mol. Opt. Phys. 47 (2014) 185002.

9. N. Aquino, Int. J. Quantum Chem. 54 (1995) 107.

10. N. Aquino, G. Campoy and H. E. Montgomery, Int. J. Quantum Chem. 107 (2007) 1548.

11. P. Harrison, Quantum Wells, Wires and Dots: Theoretical and Computational Physics of Semiconductor Nanostructures, $2^{a}$ Ed., John Wiley and Sons, Ltd., 2005.

12. T. Chakraborty, Quantum Dots: A survey of the properties of artificial atoms, (Elsevier, 1999).

13. J. Hernández-Rojas, J. Bretón, J. M. Gomez Llorente, J. Chem. Phys. 104 (1996) 1179.

14. Zhu, Y. C. Chang, Phys. Rev. B 50 (1994).

15. E. M. Corson, I. Kaplan, Phys. Rev. 71 (1947) 130.

16. S. Goldman, C. Joslin, J. Phys. Chem. 96 (1992) 6021.
17. M. Friedman, A. Rabinovitch, R. Thieberger, J. Comp. Phys. 33 (1979) 359.

18. R. J. Swenson, S. H. Danforth, J. Chem. Phys. 57 (1972) 1734.

19. Le Sech, A. Banerjee, J. Phys. B: At. Mol. Opt. Phys. 44 (2011) 105003.

20. A. Banerjee, C. Kamal, A. Chowdhury, Phys. Lett. A 350 (2006) 121.

21. J. Griffiths, Introduction to Quantum Mechanics, Prentice Hall (1995).

22. S. Saxon, Elementos de Mecánica Cuántica, Ed. Easo (1968).

23. Arfken, Mathematical Methods for Physicists, $3^{a}$ Ed., Academic Press Inc. (1985).

24. M. T. Yamashita, Revista Brasileira de Ensino de Fisica 30 (2008) 3312.

25. R. A. Rojas, Tesis de Maestría, (Universidad Autónoma Metropolitana-Iztapalapa, 2014).

26. A. S. Davydov, Quantum Mechanics, (Pergamon Press 1965).

27. S. R. Gadre, S. B. Sears, S. J. Chakravorty, R. D. Bendale, Phys. Rev. A 32 (1985) 2602.

28. M. Hô, R. P. Sagar, V. H. Smith Jr., R. O. Esquivel, J. Phys. B: At. Mol. Opt. Phys. 27 (1994) 5149.

29. E. Jaynes, Papers on Probability Statistics and Statistical Physics, Ed. Rosencrantz (Dordrecht: Reidel).

30. V. A. Sommerfeld and H. Welker, Annalen der Physik 5 (1938) 32. 
31. D. Suryanarayana and J. A. Weil, J. Chem. Phys. 64 (1976) 510.

32. N. Aquino, A. Flores-Riveros and J. F. Rivas-Silva, Phys. Lett. A, 377 (2013) 2062.

33. E. Ley-Koo and S. Rubinstein, J. Chem. Phys. 71 (1979) 351.

34. Y. P. Varshni, J. Phys. B 31 (1998) 2849.

35. J. Garza, R. Vargas and A. Vela, Phys. Rev. E 58 (1998) 3949.

36. H. E. Montgomery, Jr. Int. J. Mol. Sci. 2 (2001) 103.
37. E. Ley-Koo, Advances in Quantum Chem. 57 (2009) 84.

38. S. R. de Groot and C. A. ten Seldam, Physica XII (1946) 669.

39. P. W. Fowler, Mol. Phys. 53 (1984) 865.

40. K. D. Sen, J. Chem. Phys. 122 (2005) 194324 and references therein.

41. H. E. Montgomery Jr., N. Aquino and K. D. Sen, Int. Journal of Quantum Chem. 107 (2007) 798. 
EFECTOS DE CONFINAMIENTO ESPACIAL Y DE NÚCLEO DE TAMAÑO FINITO EN SISTEMAS ATÓMICOS.
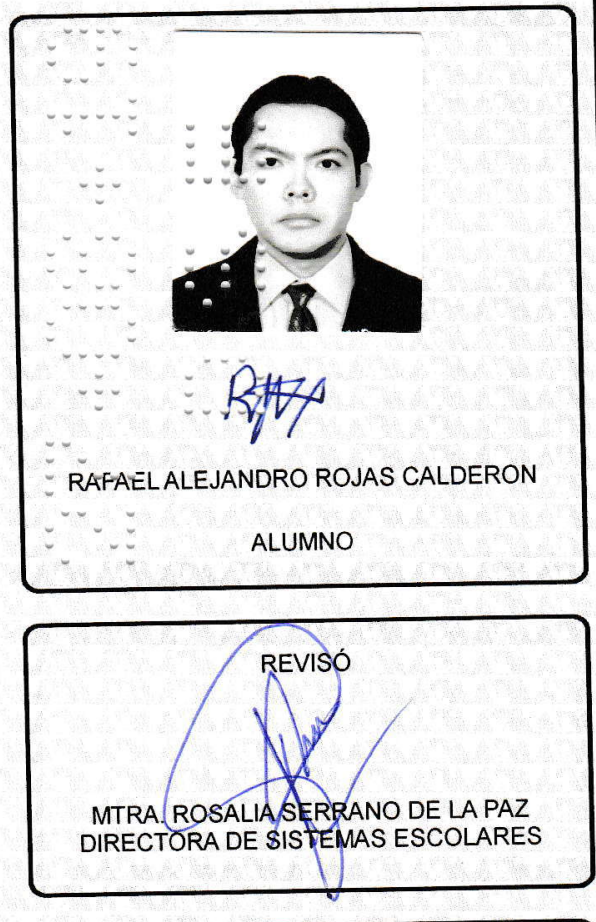

DIRECTOR DE LA DIVISIÓN DE CBI

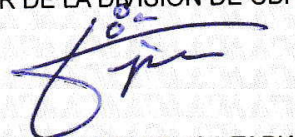

DR. JESUS ALBERTO OCHOA TAPIA
En la Ciudad de México, se presentaron a las 12:00 horas del día 24 del mes de julio del año 2019 en la Unidad Iztapalapa de la Universidad Autónoma Metropolitana, los suscritos miembros del jurado:
DR. SALVADOR ANTONIO CRUZ JIMENEZ
DR. ELEUTERIO CASTAÑO TOSTADO
DR. ANTONIO FLORES RIVEROS
DR. ANDREI SOLORZANO PEREZ
DR. NORBERTO AQUINO AQUINO

Bajo la Presidencia del primero y con carácter de Secretario el último, se reunieron a la presentación de la Disertación pública cuya denominación aparece al margen, para la obtención del grado de:

DOCTOR EN CIENCIAS (FISICA)

DE: RAFAEL ALEJANDRO ROJAS CALDERON

y de acuerdo con el artículo 78 fracción IV del Reglamento de Estudios Superiores de la Universidad Autónoma Metropolitana, los miembros del jurado resolvieron:

\section{Aprobar}

Acto continuo, el presidente del jurado comunicó al interesado el resultado de la evaluación $y$, en caso aprobatorio, le fue tomada la protesta.
VOCAL

CANCELADO

DR. ANTONIO FLORES RIVEROS

\section{PRESIDENTE}

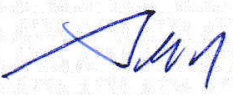

DR. SALVADOR ANTONIO CRUZ JIMENEZ

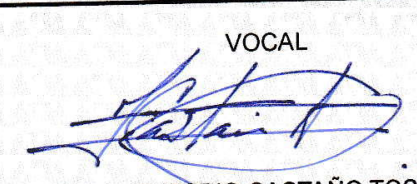

DR. ELEUTERIO CASTAÑO TOSTADO

$$
\checkmark
$$
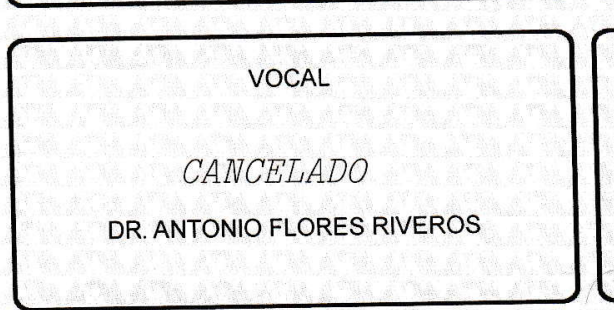

SECRETARIO

Yur qui qu

DR. NORBERTO AQUINO AQUINO 